\section{To: (Receiving Organization)}

Distribution

\section{Proj./Prog./Dept./Div.:}

\section{Originator Remarks:}

This document is being released into the supporting document system for retrievability purposes.

\section{Receiver Remarks:}

\author{
3. From: (Originating Organization) \\ Nuclear Safety and Licensing \\ 6. Design Authority/Design Agent/Cog. Engr.: \\ D. R. Bratzel
}

Nuclear Safety and Licensing

For Release

\begin{tabular}{l} 
4. Related EDT No.: \\
N/A \\
\hline 7. Purchase Order No.: \\
N/A \\
9. Equip./Component No.:
\end{tabular}

$\mathrm{N} / \mathrm{A}$

10. System/Bidg./Facility:

$\mathrm{N} / \mathrm{A}$

12. Major Assm. Dwg. No.:

$\mathrm{N} / \mathrm{A}$

13. Permit/Permit Application No.

$\mathrm{N} / \mathrm{A}$

14. Required Response Date:

$\mathrm{N} / \mathrm{A}$

\begin{tabular}{|c|c|c|c|c|c|c|c|c|}
\hline 15. & & DATA TR & NSMITTE & & (F) & (G) & $(\mathrm{H})$ & (l) \\
\hline $\begin{array}{l}\text { (A) } \\
\text { Item } \\
\text { No. }\end{array}$ & (B) Document/Drawing No. & $\begin{array}{l}\text { (C) Sheet } \\
\text { No. }\end{array}$ & $\begin{array}{l}\text { (D) Rev. } \\
\text { No. }\end{array}$ & (E) Title or Description of Data Transmitted & $\begin{array}{l}\text { Approval } \\
\text { Desig- } \\
\text { nator }\end{array}$ & $\begin{array}{l}\text { Reason } \\
\text { for Trans- } \\
\text { mittal }\end{array}$ & $\begin{array}{c}\text { Origi- } \\
\text { nator } \\
\text { Dispo- } \\
\text { stion }\end{array}$ & $\begin{array}{l}\text { Receiv- } \\
\text { er } \\
\text { Dispo- } \\
\text { sition }\end{array}$ \\
\hline 1 & $\mathrm{RPP}-6213$ & $\mathrm{~N} / \mathrm{A}$ & 0 & Hanford Waste Tank Bump & $\mathrm{N} / \mathrm{A}$ & 2 & 1 & 1 \\
\hline & & & & Accident and Consequence & & & & \\
\hline & & & & Analysis & & & & \\
\hline & & & & & & & & \\
\hline & & & & & & & & \\
\hline & & & & & & & & \\
\hline & & & & & & & & \\
\hline
\end{tabular}

16.

KEY

\begin{tabular}{|c|c|}
\hline Approval Designator $(F)$ & \\
\hline $\begin{array}{c}E, S, Q, D \text { OR N/A } \\
\text { (See'WHC-CM-3-5, } \\
\text { Sec. 12.7) }\end{array}$ & $\begin{array}{l}\text { 1. Approval } \\
\text { 2. Release } \\
\text { 3. Information }\end{array}$ \\
\hline
\end{tabular}

17.

Reason for Transmittal (G)

4. Review

5. Post-Review

6. Dist. (Receipt Acknow. Required)

SIGNATURE/DISTRIBUTION

(See Approval Designator for required signatures)

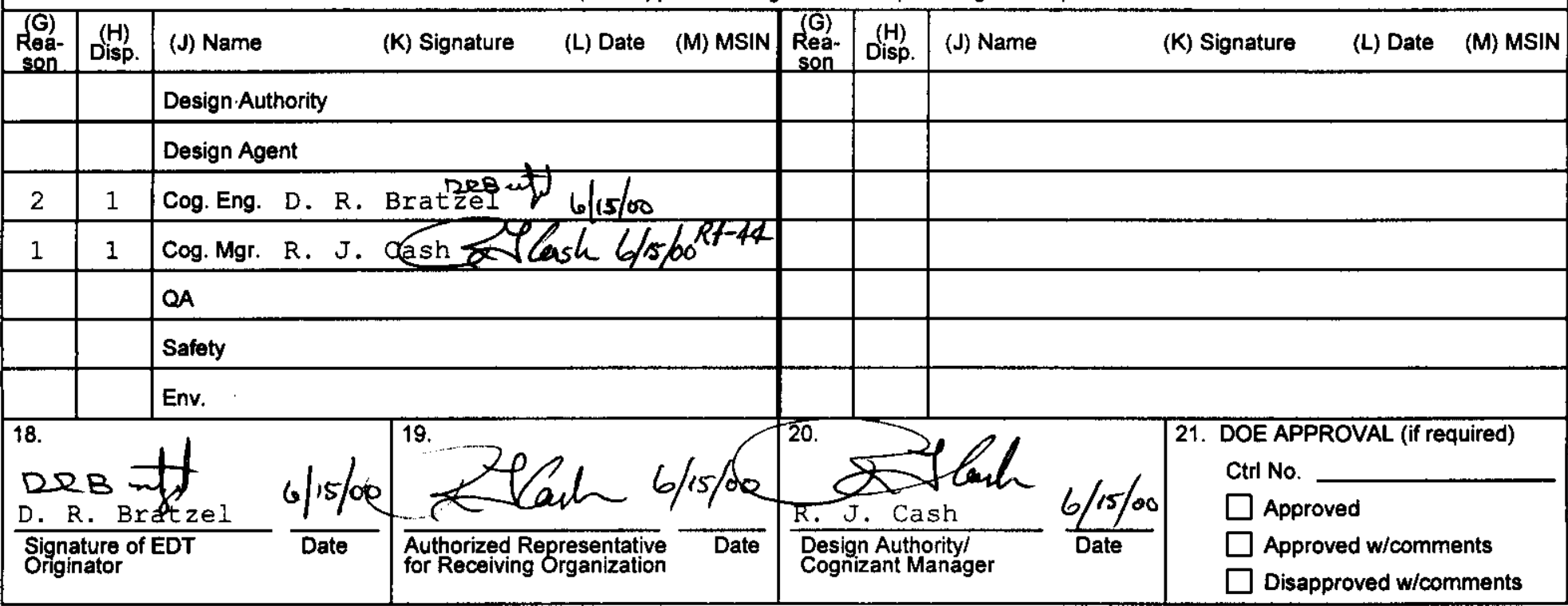




\section{DISTRIBUTION SHEET}

To

Distribution

Project Title/Work Order

RPP-6213, Rev. 0, "Hanford Waste Tank Bump Accident and Consequence Analysis"

From

Nuclear Safety \& Licensing Name

CH2M HILL Hanford Group, Inc.

D. R. Bratzel

R. J. Cash

G. W. Gault

T. G. Goetz

J. M. Grigsby

J. P. Harris

G. D. Johnson

N. W. Kirch

C. E. Leach

S. N. Maruvada

J. E. Meacham

R. G. Stickney

L. E. Thomas

Fauske \& Associates, Inc.

M. G. Plys

B. Malinovic

John Marvin, Inc.

D. M. Ogden

Fluor Federal Services

G. W. Ryan

T. B. McCall

\section{(6 Copies)}

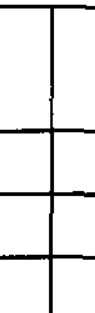

\begin{tabular}{l|c}
\hline $\mathrm{R} 1-44$ & $\mathrm{X}$ \\
\hline $\mathrm{R} 1-44$ & $\mathrm{X}$ \\
\hline $\mathrm{R} 1-44$ & $\mathrm{X}$ \\
$\mathrm{R} 1-49$ & $\mathrm{X}$ \\
\hline $\mathrm{R} 1-44$ & $\mathrm{X}$ \\
\hline $\mathrm{R} 1-49$ & $\mathrm{X}$ \\
\hline $\mathrm{R} 1-44$ & $\mathrm{X}$ \\
\hline $\mathrm{R} 2-11$ & $\mathrm{X}$ \\
\hline $\mathrm{R} 1-44$ & $\mathrm{X}$ \\
\hline $\mathrm{R} 1-44$ & $\mathrm{X}$ \\
\hline $\mathrm{R} 1-49$ & $\mathrm{X}$ \\
\hline $\mathrm{R} 1-43$ & $\mathrm{X}$ \\
\hline $\mathrm{S} 7-01$ & $\mathrm{X}$ \\
\hline
\end{tabular}

\begin{tabular}{l}
$x$ \\
\hline$x$ \\
\hline$x$ \\
\hline$x$ \\
\hline$x$ \\
\hline$x$ \\
\hline$x$ \\
\hline$x$ \\
\hline$x$
\end{tabular}

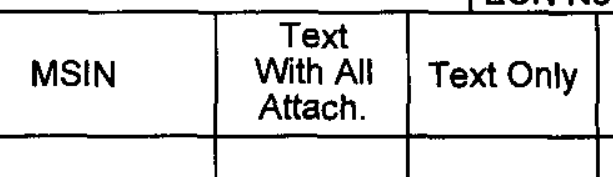

Page 1 of 1

Date

EDT No. 623348

ECN No. N/A

Attach I

Appendix Only

EDT/ECN

Only 


\title{
Hanford Waste Tank Bump Accident and Consequence Analysis
}

M. Epstein, B. Malinovic, M.G. Plys, G.M. Hauser, Fauske A Associates D. R. Bratzel, CH2M HILL Hanford Group, Inc.

Richland, WA 99352

U.S. Department of Energy Contract DE-AC06-99RL14047

\author{
EDT/ECN: 623348 \\ UC: $\quad \mathrm{N} / \mathrm{A}$ \\ Cost Center: 74 F00 \\ B\&R Code: $\quad$ EWO/2J2020 \\ Charge Code: 109311AA30 \\ Total Pages: 232
}

Key Words: Tank Bump, Accident Analysis, Consequence Analysis

Abstract: This report provides a new evaluation of the Hanford tank bump accident analysis and consequences for incorporation into the Authorization Basis. The analysis scope is for the safe storage of waste in its current configuration in single-shell and double-shell tanks.

TRADEMARK DISCLAIMER. Reference herein to any specific commercial product, process, or service by trade name, trademark, manufacturer, or otherwise, does not necessarily constitute or imply its endorsement, recommendation, or favoring by the United States Government or any agency thereof or its contractors or subcontractors.

Printed in the United States of America. To obtain copies of this document, contact: Document Control Services, P.O. Box 950, Mailstop H6-08, Richland WA 99352, Phone (509) 372-2420; Fax (509) 376-4989.
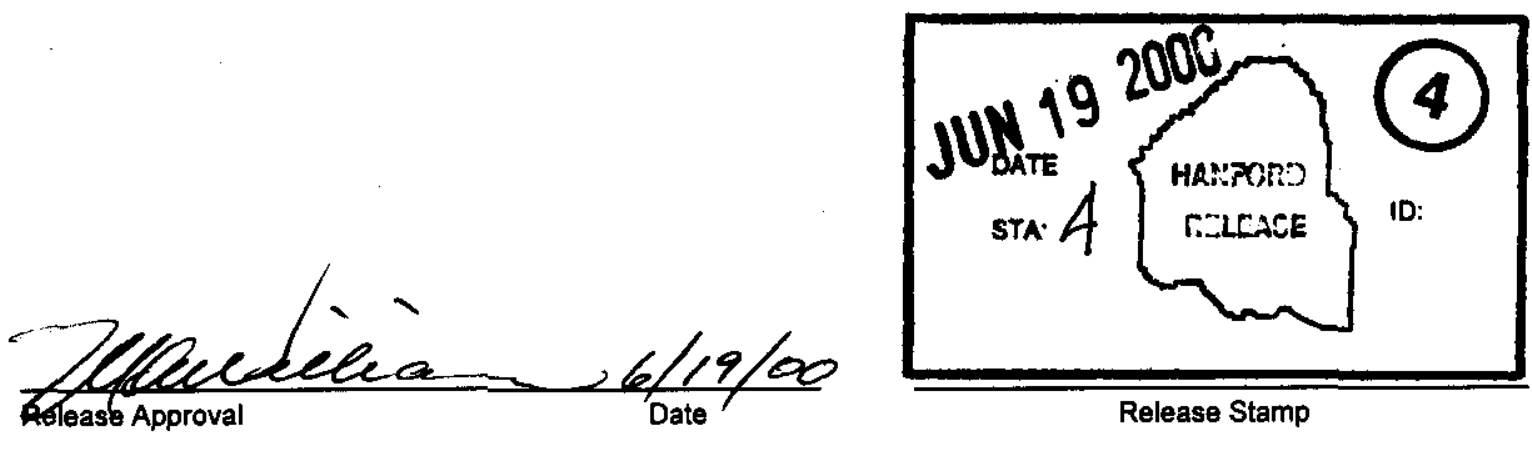

\section{Approved For Public Release}


RPP-6213

Revision 0

\section{HANFORD WASTE TANK BUMP ACCIDENT AND CONSEQUENCE ANALYSIS}

Prepared for:

D. R. Bratzel

CH2M HILL Hanford Group, Inc.

Prepared by:

M. Epstein, B. Malinovic, M. G. Plys, \& G. M. Hauser

Fauske \& Associates, Inc.

16W070 West $83^{\text {rd }}$ Street

Burr Ridge, Illinois 60521

Date Published

June 2000

Prepared for the U.S. Department of Energy

Office of River Protection

Approved for public release, distribution unlimited 


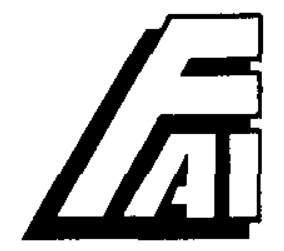

FAI/00-14, Rev. 0

HANFORD WASTE TANK BUMP ACCIDENT AND CONSEQUENCE ANALYSIS

\author{
Submitted To: \\ CH2M HILL Hanford Group, Inc. \\ 2355 Stevens Center \\ Richland, Washington 99352
}

\author{
Prepared By: \\ M. Epstein, B. Malinovic, M. G. Plys, \& G. M. Hauser \\ Fauske \& Associates, Inc. \\ 16W070 West 83rd Street \\ Burr Ridge, Illinois 60521 \\ Tel: (630) 323-8750 Fax: (630) 986-5481
}

May 2000 
RPP-6213 REV 0

This page intentionally left blank. 
This page cannot be converted.

Please view the native document

for the original page. 


\section{TABLE OF CONTENTS}

(continued)

Page

6.0 BUMP CRITERIA MODEL APPLICATION

6-1 of 14

6.1 Application of Tank Bump Criteria to Current Tank Waste .............................6-1

6.2 Application of Tank Bump Criteria to Waste at LCO Conditions .....................6-8

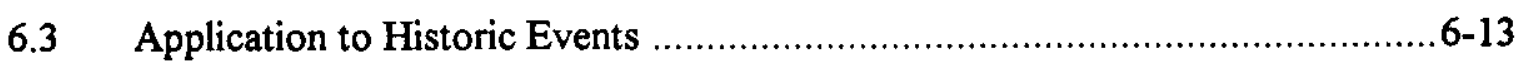

6.4 Note on Screening Tank Bump Potential ................................................. 6-13

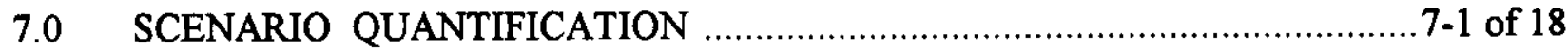

7.1 Ventilation System Failure and Failure to Recover ......................................... 7-1

7.1.1 Failure to Recover Probability ........................................................

7.1.2 Ventilation System Failure Frequency ……...................................7-6

7.1.3 Tank Bump Conditions Frequency for LCO and Current Conditions ..7-7

7.2 Loss of Off-Site Power Frequency …….................................................... 7-8

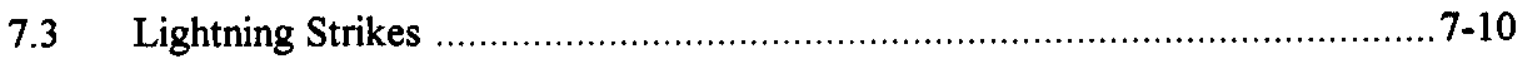

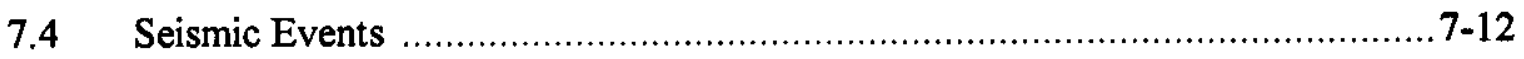

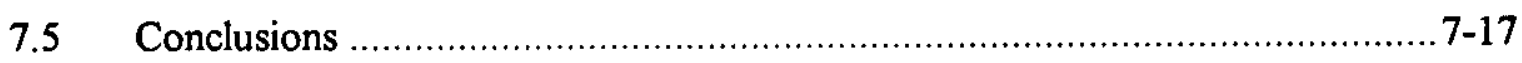

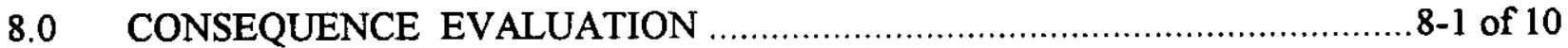

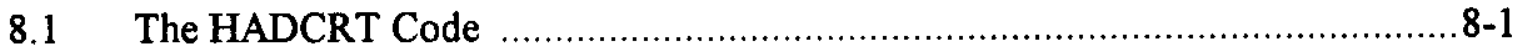

8.2 HADCRT Input for 241-AZ-102 Case ……............................................. 8-2

8.3 241-AZ-102 Tank Bump Consequence Physical Results .................................8-4

8.4 241-AZ-102 Tank Bump Radiological and Toxic Chemical Consequences ........ 8-4

9.0 SUMMARY AND DISCUSSION OF ASSUMPTIONS AND

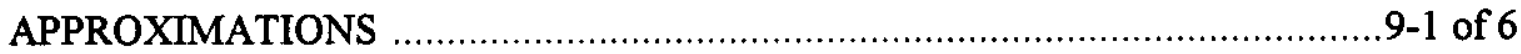

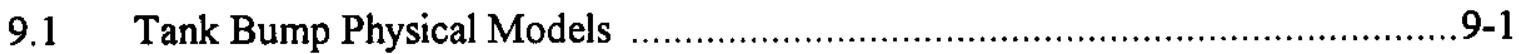

9.2 Tank Bump Frequency Calculation .........................................................

9.3 Tank Bump Consequence Evaluation ...................................................... 9-4

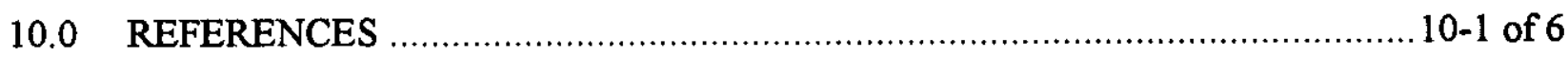

APPENDIX A Tank Waste Characterization Data ……................................ A-1 of 24

APPENDIX B Postulated Bump Scenarios Deemed Not Plausible .................. B-1 of 12 


\section{TABLE OF CONTENTS}

(concluded)

Page

APPENDIX C APPENDIX D APPENDIX E APPENDIX F APPENDIX G APPENDIX $\mathrm{H}$ APPENDIX I APPENDIX J
Rise Distance to Yielding of Void-Bearing Parcel C-1 of 6 Bubble Growth Model and Input D-1 of 6 Cluster Entrainment Model and Input E-1 of 8 HADCRT Changes and Input F-1 of 24 Spreadsheets for Bump Criteria G-1 of 6 Spreadsheet for Scenario Quantification .H-1 of 12 Quality Assurance Documents I-1 of 4 Checklist for Technical Peer Review J-1 of 4 


\section{LIST OF FIGURES}

Figure

3-1 Surface Heat Removal ...............................................................................

3-2 Cavity Expansion During Rise in a Constant Temperature Pool ...........................3-7

3-3 Best-Estimate Initial Temperature Profile for $11 \mathrm{ft}$. of Consolidated Non-

Convective Waste

3-4 Conservative Initial Temperature Profile for $11 \mathrm{ft}$. of Consolidated NonConvective Waste

3-5 Gas Volume Fraction Contours for GOTH Run With 18" Non-Convective Layer and No Condensation

3-6 Dimensional HEATING7 Model (Waters et al. 1991) ...................................... 3-16

3-7 Single-Shell Tank (SST) Instrumentation Configuration ................................. 3-20

3-8 Double-Shell Tank (DST) Instrumentation Configuration ...................................3-21

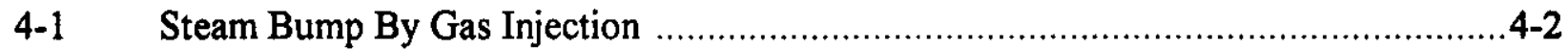

4-2 Steam Bump During a Buoyant Displacement .............................................. 4-3

4-3 Bubble Expansion Ratio Versus Depth of Supernatant Pool; Pool Temperature

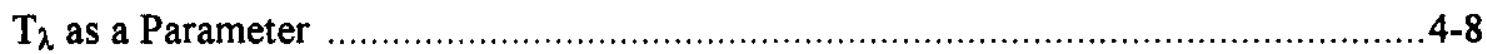

4-4 Bubble Expansion Ratio Versus Depth of Supernatant Pool; Initial Bubble Diameter as a Parameter

4-5 Bubble Expansion Ratio Versus Convective Layer Temperature $\left(<100^{\circ} \mathrm{C}\right)$ or Buoyant Parcel Temperature $\mathrm{T}_{\mathrm{nc}}$ During Buoyant Displacement

5-1 Sequence of Events in Model of Steam Bump and Liquid Waste Release

5-2 Temperature History of Buoyant Parcel Compared With its Boiling Temperature; Open Headspace

5-3 Temperature History of Buoyant Parcel Compared With its Boiling Temperature; Sealed Headspace 


\section{LIST OF FIGURES}

(continued)

6-1 241-AZ-101 Heatup After Loss of Ventilation for Current Conditions $6-6$

6-2 241-AZ-102 Heatup After Loss of Ventilation for Current Conditions

6-3 241-AZ-101 Heatup After Loss of Ventilation - LCO Conditions 6-11

6-4 241-AZ-102 Heatup After Loss of Ventilation - LCO Conditions $6-12$

7-1 Calculated Reverse Cumulative Distribution for Tank Bump Frequency . $7-9$

7-2 Loss of Off-Site Power (LOSP) Frequency of Duration 7-11

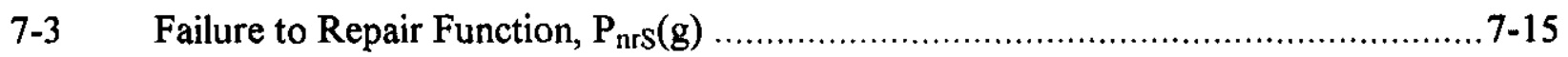

7-4 Distribution for the Frequency of Tank Bumps Induced by Seismic Events .............7-16

8-1 241-AZ-102 Tank Bump Short-Term Results ........................................................ 8-5

8-2 241-AZ-102 Tank Bump Long-Term Results …................................................ 8-6

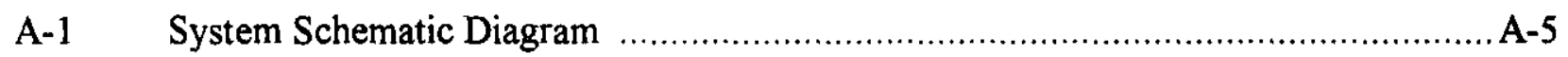

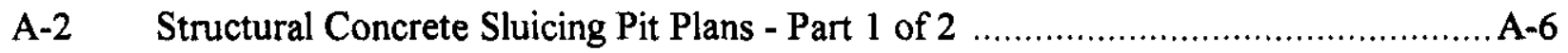

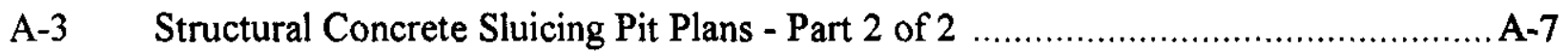

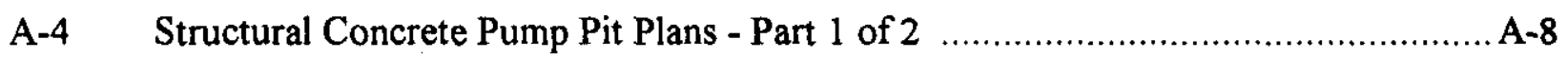

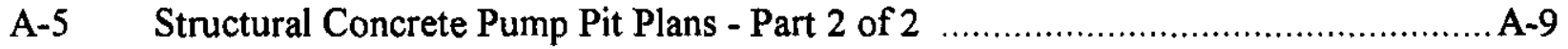

A-6 Air Lift Circulator and Riser Extension Details - Part 1 of $2 \ldots \ldots \ldots \ldots \ldots \ldots \ldots \ldots \ldots \ldots \ldots . . . . . . .10$

A-7 Air Lift Circulator and Riser Extension Details - Part 2 of $2 \ldots \ldots \ldots \ldots \ldots \ldots \ldots \ldots \ldots \ldots \ldots . . . . .1-11$

B-1 Steam Bump Due to Interstitial Superheat in Non-Convective Layer (Hypothetical)

B-2 Dimensionless Liquid Superheat Versus Dimensionless Time …............................ B-5 
RPP-6213 REV 0

\section{LIST OF FIGURES}

(concluded)

Figure

$\underline{\text { Page }}$

B-3 Steam Bump Due to Local Convection in Non-Convective Layer

(Hypothetical, "Fumarole")

B-8

B-4 Wave Growth Time Versus Disturbance Wave Length; Depth of Less-Dense

Layer as a Parameter

C-1 Rise Distance to Yielding of Void-Bearing Parcel Versus Temperature of NonConvective Layer; Input Parameters Pertain to Tank 241-AZ-102 


\section{LIST OF TABLES}

Table

Page

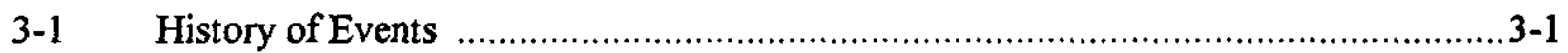

3-2 Waste Properties for GOTH Analysis (Sathyanarayana 1996) .................................3-9

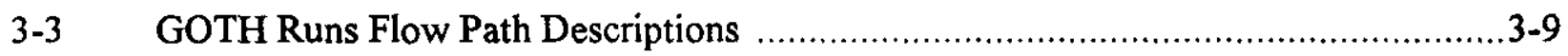

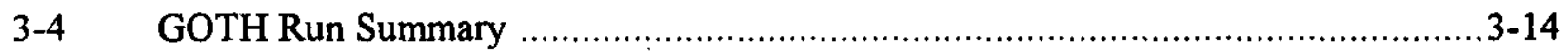

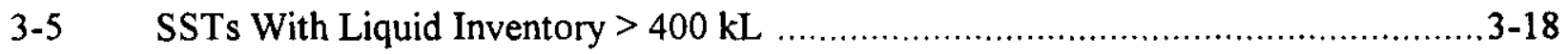

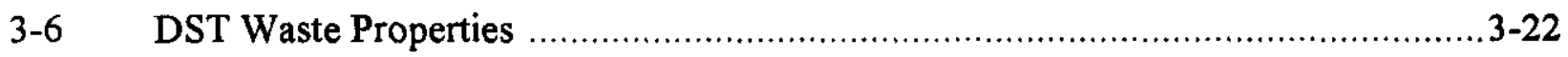

4-1 Gas and Steam Generation Rates in the Non-Convective Layer ........................... 4-13

4-2 Buoyant Displacement Model Predictions for the High-Power Double-Shell

Tanks

6-1 DSTs With Negligible Non-Convective Layer (Hu et al. 2000) ….........................6-2

6-2 SSTs and DSTs With Small Heat Load and/or Small Supernatant Depth (Hu et al. 2000)

6-3 Time to Saturated Conditions for DSTs With Significant Supernatant Depth \&

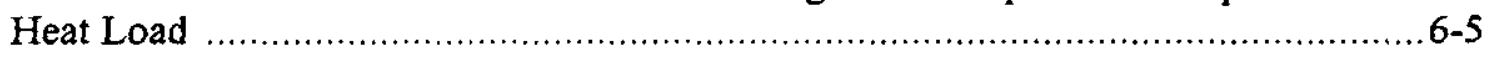

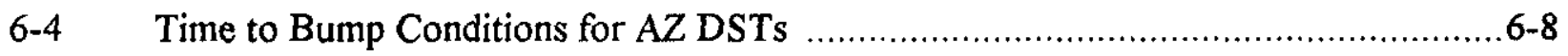

6-5 Time to Saturated Conditions for DSTs Under LCO ............................................. 6-9

6-6 Time to Saturated Conditions for AZ DSTs Under LCO Conditions ..................... 6-10

7-1 Critical Items List for the High Level Waste Transfer RAM B, Ranked by the Contribution of System Type to Expected Schedule (Carlson 1999a)

7-2 Component Types and Recovery Conditions (Carlson 1999) ................................... 7-4

7-3 Summary of Corrective Maintenance (CM) Requirements (Clock Hours and Effort) (Carlson 1999) ….................................................................

7-4 Corrective Maintenance Activity Clock Time Distributions .................................. 7-6

FAI/00-14, Rev. $0 \quad-i x-$ 
RPP-6213 REV 0

\section{LIST OF TABLES}

(concluded)

Table

Page

7-5 Seismic Hazard Curve Data for the Hanford Site 7-12

8-1 Parameter Values for Consequence Analysis Example for Tank 241-AZ-102 ..........8-3

8-2 Radiological Consequence Factors (Cowley et al. 2000) ...................................8.7

8-3 Sum-of-Fraction of Risk Guidelines for a Unit Release of Chemicals

(WHC-SD-WM-SARR-011 1996)

8-4 Toxic Consequence Evaluation Results ......................................................

C-1 Parameter Values for the Buoyant Parcel Yielding Criterion (Tank 241-AZ-102) ..... C-6 


\subsection{PURPOSE AND SCOPE}

This report provides a new evaluation of the Hanford tank bump accident analysis (HNF-SD-WM-SAR-067 2000). The purpose of the new evaluation is to consider new information and to support new recommendations for final safety controls. This evaluation considers historical data, industrial failure modes, plausible accident scenarios, and system responses.

A tank bump is a postulated event in which gases, consisting mostly of water vapor, are suddenly emitted from the waste and cause tank headspace pressurization. A tank bump is distinguished from a gas release event in two respects: First, the physical mechanism for release involves vaporization of locally superheated liquid, and second, gases emitted to the head space are not flammable. For this reason, a tank bump is often called a steam bump. In this report, even though noncondensible gases may be considered in bump models, flammability and combustion of emitted gases are not.

The analysis scope is safe storage of waste in its current configuration in single-shell tanks (SSTs) and double-shell tanks (DSTs). The analysis considers physical mechanisms for tank bump to formulate criteria for bump potential, application of the criteria to the tanks, calculation of expected bump frequency in terms of events per year, and consequence analysis of bump scenarios. The result of consequence analysis is the mass of waste released from tanks for specific scenarios where bumps are credible; conversion to health consequences is performed using standard Hanford methods (Cowley et al. 2000; and WHC-SD-WM-SARR-011 1996). The analysis forms a baseline for future extension to consider waste retrieval. 
RPP-6213 REV0

This page intentionally left blank. 


\subsection{SUMMARY OF FINDINGS}

Postulated physical scenarios leading to tank bumps are comprehensively examined here. We conclude that a combination of a substantial supernatant layer depth, supernatant temperatures close to saturation, and commensurate sludge temperatures are required for a tank bump to occur. We have ruled out scenarios postulated at various times for sludge layers lacking substantial supernatant, such as superheat within the layer and fumarole formation leading to a bump.

A graded set of criteria is presented to screen tanks for bump potential. These screening criteria may be applied as tank waste inventories are changed. Bump scenarios and criteria can explain observations of historical bump events and show the difference in tank bump potential earlier in tank life compared to the present day, essentially due to high historical tank power and in some cases gas injection.

Applying criteria for non-negligible supernatant layers and non-negligible non-convective layers, all but four SSTs in their present state may be considered immune to a tank bump, as can some DSTs. Simple screening for tank power removes the remaining SSTs and many DSTs from consideration. Evaluation of time to attain saturation shows that three tanks, 241-AZ-101, 241-AZ-102, and 241-AY-102, are the only tanks for which a bump is theoretically possible on that basis. However, these tanks fail the final screening criterion: They cannot have buoyant displacement initiated by noncondensible gas production. Indeed, these tanks are not observed to have buoyant displacements that lead to gas release events nor are they predicted to have them at high sludge temperatures. Therefore, in our best judgement, no SSTs and no DSTs can have a tank bump event during safe storage.

Extended loss of cooling is required to develop initial conditions sufficient for a tank bump during safe storage. Here we consider the frequency of tank bump for 241-AZ-101, 241-AZ-102, and 241-AY-102, even though these tanks fail the final bump screening criterion. Considering a range of initiating events and failure to recover tank cooling and present waste temperatures, the frequency of tank bump is estimated to be extremely unlikely $\left(10^{-6} / \mathrm{yr}\right.$ to $10^{-4} / \mathrm{yr}$ ), however, this conclusion is dominated by high-acceleration seismic events. If seismic events are excluded, the frequency of tank bump is estimated to be beyond extremely unlikely (less than $10^{-6} / y r$ ). When these three tanks are operated up to the LCO limits, the frequency of tank bump is about $10^{-4} / \mathrm{yr}$, the dividing line between the unlikely and extremely unlikely frequency ranges.

A consequence evaluation for a hypothetical tank bump in tank 241-AZ-102 is therefore presented, recognizing that the required noncondensable gas accumulation is not present as an initiator, but that the external initiator frequency exceeds $10^{-6} / \mathrm{yr}$. Consequences are evaluated using a best-estimate model approach with an experimental basis for separate phenomena, including models for the amount of material released, consideration of successive releases, and mixing of formerly non-convective material with supernatant. Successive bumps are estimated to occur over approximately half-hour intervals with about $1 / 12$ the non-convective layer released per bump. An average aerosol release to headspace of about $1.8 \mathrm{~kg}$ per bump is calculated, and long-term release of about $5.2 \mathrm{~kg}$ is predicted, with about $16.2 \mathrm{~kg}$ retained in the tank. 


\section{RPP-6213 REV 0}

The mass of entrained material was converted to dose following the method of Cowley et al. (2000). An on-site dose of $0.05 \mathrm{~Sv}$ was calculated for worker exposure at $100 \mathrm{~m}$ during an 8-hour shift. An off-site dose of $1.2 \times 10^{-4} \mathrm{~Sv}$ was calculated for an 8-hour event. Toxic chemical consequences are all acceptable.

We emphasize that in our best judgement, for current safe storage, no SSTs and no DSTs can physically have a tank bump, and that the frequency of required thermal conditions is beyond extremely unlikely for present storage conditions when seismic events are excluded. 


\subsection{HISTORICAL AND PRESENT EVALUATION DATA}

\subsection{HISTORICAL EVENTS}

Since the early 1950's, aging waste has been stored in underground storage tanks at the Hanford site. In the mid-1950's, a tank bump was first observed, which led to installation of air lift circulators (ALC) that provide liquid agitation and solid suspension. Air lift circulators, also referred to as air lift pumps, have successfully prevented bumps, and the few bumps that have occurred since the mid-1950's were initiated by ALC shutdown and restart. Discussion below presents a brief history of the documented events, which are listed in Table 3-1.

Table 3-1. History of Events.

\begin{tabular}{|c|c|c|c|c|c|c|c|c|c|c|c|}
\hline $\begin{array}{l}\text { Tank, } \\
\text { 241- }\end{array}$ & Date & $\begin{array}{l}\text { Heat } \\
\text { Rate } \\
(\mathbf{K w})\end{array}$ & $\begin{array}{c}\text { Waste } \\
\text { Temp. } \\
\text { at } \\
\text { Bottom } \\
\text { ('F) }\end{array}$ & $\begin{array}{l}\text { Dome } \\
\text { Pressure } \\
\text { (psig) }\end{array}$ & $\begin{array}{c}\text { Duration } \\
\text { (min) }\end{array}$ & $\begin{array}{c}\text { No. of } \\
\text { Documented } \\
\text { Events }\end{array}$ & ALC & Initlator & $\begin{array}{l}\text { Contam- } \\
\text { Ination }\end{array}$ & $\begin{array}{l}\text { Vent } \\
\text { System }\end{array}$ & Ref. \\
\hline S-104 & $\begin{array}{l}10 / 53 \\
-5 / 54\end{array}$ & 200 & $>240$ & $<1.6$ & 8 to 42 & Many & 0 & None & Yes & No & {$[1]$} \\
\hline S-101 & $\begin{array}{l}1 / 54- \\
8 / 54\end{array}$ & 200 & $>240$ & $<1.6$ & 8 to 42 & Many & 0 & None & Yes & No & {$[1]$} \\
\hline SX-101 & $\begin{array}{l}2 / 55- \\
4 / 55\end{array}$ & 400 & 300 & $0.7-1.8$ & 3 to 13 & $>40$ & Auger & None & Unknown & No & [1] \\
\hline SX-104 & $7 / 55$ & 600 & 290 & Unknown & .70 & 2 & $\begin{array}{l}\text { Proto- } \\
\text { type }\end{array}$ & $\begin{array}{c}\text { AlC } \\
\text { shutdow } \\
\text { n/restart }\end{array}$ & Unknown & No & [2] \\
\hline SX-114 & $8 / 58$ & 650 & 357 & 2.6 & Unknown & 4 & 4 & $\begin{array}{c}\text { AlC } \\
\text { shutdow } \\
\text { n/restart }\end{array}$ & Yes & Yes & [3] \\
\hline A-105 & $1 / 65$ & 2300 & 310 & 1.8 & 30 & 1 & 4 & $\begin{array}{l}\text { Bottom } \\
\text { of tank } \\
\text { bulged }\end{array}$ & Yes & Yes & - \\
\hline$A X-101$ & $\begin{array}{l}1968- \\
1969\end{array}$ & 2300 & 260 & Unknown & 20 & 1 & 22 & $\begin{array}{c}\text { ALC } \\
\text { shutdow } \\
\text { n/restart }\end{array}$ & No & Yes & [1] \\
\hline & $411 \%$ & $d x$ & 1989) & & $121 \%$ & (1988) & & (3) & Oones 1 & 1 & \\
\hline
\end{tabular}

In a passage describing waste tank operations history, Jo (1990) states that prior to August of 1952 , conduction to underlying soil prevented boiling. In addition, air cooled heat exchangers for the S tanks were installed above ground. During August of 1952, tank 241-S-1 10 boiled and water was sprayed on the air condensers, which was sufficient to condense vapor. In 1953, tank 241-S-107 also boiled, but because water spray could not keep up with the boiling rate, a water jacket was installed on the air condenser. In both cases, contamination was not found.

According to Tomlinson (1955), "In July, 1953, tank 241-S-104 was filled and its contents started boiling at a rate which exceeded the heat exchange capacity of the water-jacketed air condensor." Despite installation of auxiliary water cooled condensers on tanks 241-S-104 and 241-S-101 circa August 1953, occasional mild eruptions were observed, followed by increased evaporation rates and contamination. Eruptions were preceded by disturbing the waste in some cases, and in other cases by no observed cause. In January of 1954, "bumping" eruptions, in the form of a series of 5 to 25 abrupt pressure surges at roughly 100 second intervals, first took place 
in tank 241-S-101. Bump initiation was concomitant with a temperature at the bottom of the tank greater than $240^{\circ} \mathrm{F}$ - the recorder temperature limit. The same phenomena were observed in tank 241-S-104, including the greater than $240^{\circ} \mathrm{F}$ temperature at the tank bottom. Tank overpressure never exceeded $1.6 \mathrm{psig}$. The two tanks were boiled down to approximately halfvolume and refilled, which suggests the tanks were nearly full when the bumps first started. Bumps continued for another eight months and then gradually diminished to a simmer.

From May 1954 to February 1955, Redox waste (excepting coating waste) filled the 241-SX-101-102-103 cascade. In February 1955, bumping was observed in tank 241-SX-101. It consisted of 5 to 25 abrupt pressure surges at intervals of about 30 seconds, with maximum pressures of $0.7 \mathrm{psig}$ to $1.8 \mathrm{psig}$. These pressure surges occurred about once per day naturally, but could be initiated two or three times daily by starting an auger installed in the tank. Tank liquid level fell from $30 \mathrm{ft}$ to $25 \mathrm{ft}$ during the next two months, even though no waste was added.

In June of 1955, tank 241-SX-104 was boiling but no bumps occurred. Boiling can be distinguished from a tank bump because boiling is gentle by comparison and involves only the supernatant rather than the settled solids. To prevent bumps, the prototype air lift circulator was installed. Initial ALC operation increased the condensate flow rate from the normal $3 \mathrm{gpm}$ to $7 \mathrm{gpm}$, but only for about six hours. On July 14, 1955, the ALC became inoperative because of a failed gasket (Hanson 1955). Upon ALC restart on July 15, 1955, a bump occurred, resulting in an instantaneous increase in the condensate flow rate from $0.8 \mathrm{gpm}$ to $17.2 \mathrm{gpm}$; the bump lasted for about 70 minutes and released heat at a rate of $4.7 \times 10^{6} \mathrm{Btu} / \mathrm{hr}$, or $1.38 \mathrm{MW}$. The bumping was attributed to the interruption in ALC operation (Hanson 1955).

Tank 241-SX-114 bumped four times during August of 1958. During the week of August 18, 1958, 241-SX-114 tank temperature gradually increased, despite the operation of four ALC at $10 \mathrm{cfm}$ each. On August 22, operators shut off air and ran $2 \mathrm{cfm}$ of water through each of the ALC. By this time, waste temperature was $357^{\circ} \mathrm{F}$. The tank bumped three times during the next couple of days, and once on August 25. In Harmon (1958), the bumps are attributed to turning off the ALC in tank 241-SX-114. Contamination was extensive both inside and outside the tank farm, although no site employees were exposed (Harmon 1958). Bumps in tank 241-SX-114 ejected "contaminated vapors" out of the tank 241-SX-113 pump pit. Contamination within the tank farm spread over about $15,000 \mathrm{ft}$, with a maximum dose rate of $5 \mathrm{Rads} / \mathrm{hr}$.

The most publicized event occurred in January of 1965, in tank 241-A-105. This bump is attributed to evaporation of water trapped in between the liner and concrete floor. Waste temperature was high enough that the vapor pressure just underneath the liner exceeded the hydrostatic head of the waste. In effect, the liner bulge was responsible for the bump, which means that this event is not applicable to the current analysis.

In an internal memorandum, Bendixsen (1989) reports that tank 241-AX-101 bumped once between 1968 and 1969. The event was never recorded because there was no finding of any contamination near the tank. Waste temperature was estimated to be $260^{\circ} \mathrm{F}$. ALC shutdown and restart was identified as the event initiator.

These events led to three historically postulated scenarios for bump initiation (Jo 1990): (1) an organic crust, (2) liner bulge, and (3) ALC restart. In the first scenario, an organic crust forms 
over the supernatant and causes pressurization and super-heating. When the pressure is large enough to crack the crust, the supernatant is released until it depressurizes and the boiling point returns to normal. Single-shell liner instability was known prior to the 241-A-105 event (Brownell 1958). In several of the events described above, ALC restart after a long period resulted in vapor generation by heat released from the superheated waste. The organic crust and liner bulge scenarios are of historical interest only, and for current operations, only the ALC restart scenario has been retained.

Given current aging waste tank operations, the following tank bump scenario was accepted in 1990 as the only credible one (Jo 1990):

- Initiator: the initiator is an equipment failure that allows particle settling or loss of heat removal,

- Heat build-up: a temperature rise in the solid and/or liquid phase caused by decay heat,

- Heat release: ALC or mixer pumps agitate superheated solids and/or liquid, thereby releasing stored heat,

- Vapor generation: superheated liquid rises and rapidly generates vapor,

- Environmental release: vapor generation is rapid enough to overwhelm the tank ventilation system, pressurize the tank, and release vapor and particles to the environment through unfiltered pathways.

Discussion below proceeds with this scenario in mind.

\subsection{PREVIOUS ANALYSIS}

Previous analyses consist of the Fauske and Associates, Inc. reports of 1989 (FAI 1989) and 1999 (Epstein et al. 1999; and Waters et al. 1991), and the GOTH analyses carried out by Westinghouse Hanford Company (WHC) (Sathyanarayana 1996). The 1989 Fauske and Associates, Inc. document (FAI 1989) presents conclusions inferred from the documented events and supported by relatively straightforward hand calculations for the several bump heat transfer phenomena. As part of the technical basis for safe storage after sluicing and cessation of water addition, Epstein's et al. (1999) report considers the steam bump potential in tank 241-C-106, although the conclusions drawn in it can be applied generally. Using HEATING7, Waters et al. (1991) performed a series of parametric calculations to determine tank bump potential during-in-tank washing operation proposed for the AZ tanks. HEATING7 is a multidimensional code for finite difference solutions of the conduction equation in cylindrical, spherical, or rectangular coordinate systems. The WHC report (Sathyanarayana 1996) contains parametric calculations based on the GOTH code, which was derived from EPRI's GOTHIC code. GOTH is a finite volume program that solves the transient equations expressing mass, momentum, and energy conservation for multi-component, multiphase fluid flow. The FAI (1989) report is considered first, as it provides a succinct overview of tank bump phenomenology. 


\subsubsection{Fauske and Associates, Inc., 1989}

In December of 1989, Fauske and Associates, Inc. issued an Independent Review of Aging Waste Tank "Bump" Phenomena, which considered the background material on tank bumps and presented new observations regarding bump heat transfer mechanisms (FAI 1989). Some of the conclusions stated in the report could be inferred from the documented events, but hand calculations were presented to characterize several bump heat transfer mechanisms. The report's conclusions are as follows:

- Tank bumps require that the pool surface reach the boiling temperature,

- Tank dome ventilation should be sufficient to keep pool surface temperature below the boiling temperature,

- $\quad$ Setting aside ALC restart, it is likely that the waste depth must exceed some minimum value for the tank bump to occur, and

- There is probably some minimum tank power level below which tank bumps cannot naturally occur, regardless of pool surface temperature.

To augment the basis for these conclusions, phenomena considered by hand calculation include surface evaporation, pool circulation, dryout in settled beds, and gas injection on restart of ALC. A surface evaporation calculation was used to show tank ventilation should be sufficient to keep pool surface temperature below the boiling temperature. By the evaporation heat transfer analogy of Epstein (1988), latent and sensible heat transfer from the pool to some sink temperature were computed as a function of pool surface temperature, $T_{s}$, assuming that water mass fraction at infinity was zero and the water mass fraction at the surface was related to vapor pressure at $T_{s}$. The results shown in Figure 3-1 indicate that surface evaporation can remove a pool heat rate of $200 \mathrm{~kW}$ at a surface temperature of only 35 to $40^{\circ} \mathrm{C}$, if the dome has low relative humidity.

To demonstrate that convective motion in the pool is virtually certain, the Rayleigh number was calculated as follows:

$$
R_{a}=\frac{g^{4} \beta \Delta T}{h \vee \alpha}
$$

where $\mathrm{g}$ is the acceleration of gravity, $\mathrm{d}$ is the pool diameter $(23 \mathrm{~m}), \beta$ is the thermal expansion coefficient for water $\left(6 \times 10^{-4} \mathrm{~K}^{-1}\right), \mathrm{h}$ is the height of the pool $(10 \mathrm{~m}), v$ is the liquid kinematic viscosity $\left(3 \times 10^{-5} \mathrm{~m}^{2} / \mathrm{s}\right)$, and $\alpha$ is the liquid thermal diffusivity $\left(3 \times 10^{-5} \mathrm{~m}^{2} / \mathrm{s}\right)$. If the temperature difference is only $1^{\circ} \mathrm{C}, \mathrm{Ra}=10^{15}$. This is orders of magnitude higher than the Rayleigh number required for the onset of convection, which is somewhere between 100 and 10,000 , depending on the thermal boundary conditions. The report then goes on to conclude that natural convection should suffice to keep the pool isothermal and that dome relative humidity determines bulk pool temperature. This conclusion means that a submerged air supply is not absolutely necessary. 
Figure 3-1. Surface Heat Removal.

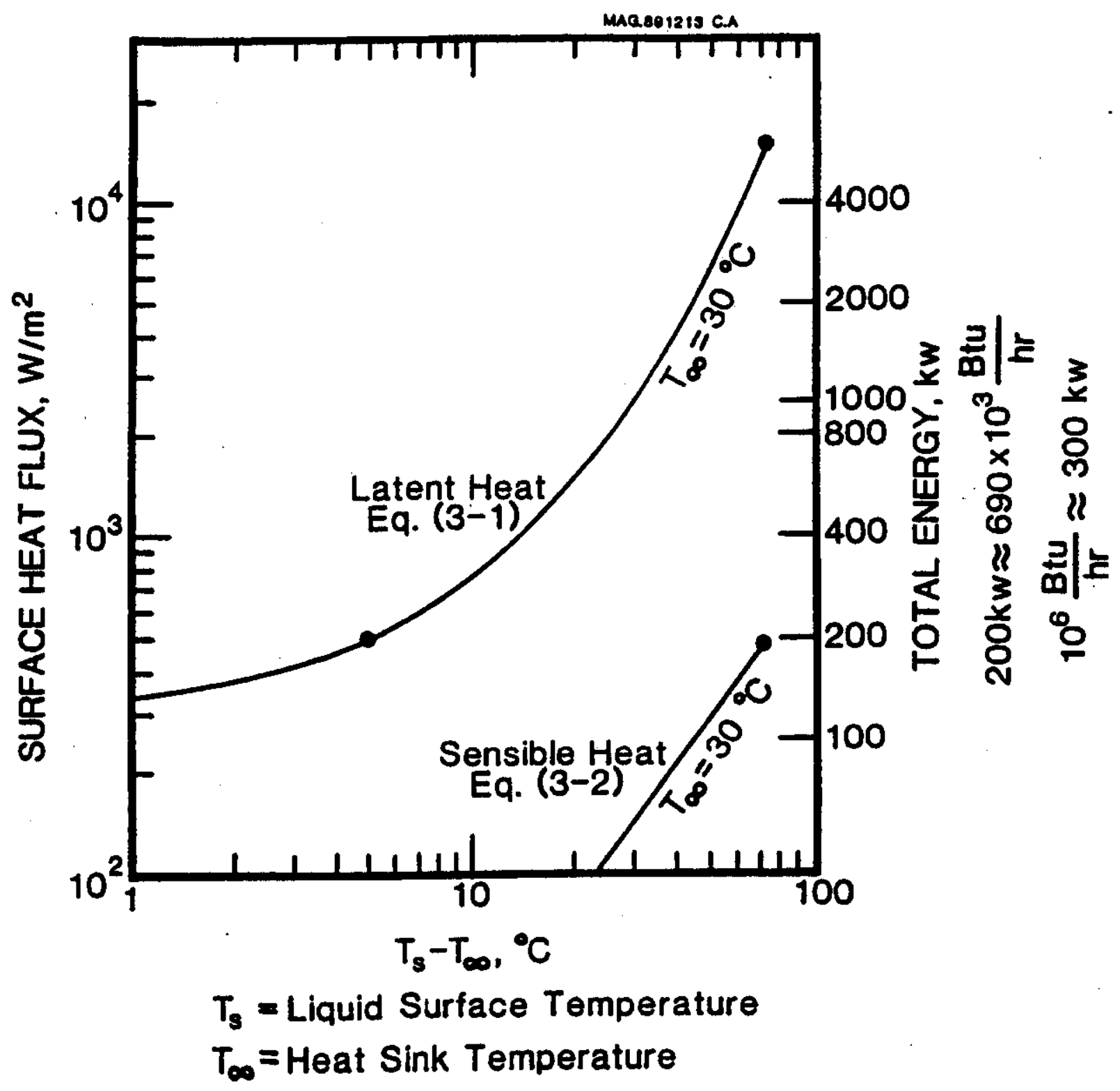


Phenomena relating to gas injection on restart of ALC were considered in both FAI (1989) and Epstein et al. (1999). Consider the ascent of a cavity volume in an isothermal pool. A relationship for the relative volume (local volume divided by initial cavity volume, $V_{0}$ ) as a function of height is:

$$
\frac{\mathrm{V}}{\mathrm{V}_{\mathrm{o}}}=\frac{\beta+1}{\beta+\left(1-\mathrm{H}^{*}\right)}
$$

where $\mathrm{H}^{*}=1-\mathrm{H} / \mathrm{H}_{\mathrm{o}}$ is the dimensionless height, $\mathrm{H}_{\mathrm{o}}$ is the pool depth, and the term $\beta=$ $\left[\mathrm{P}_{\text {surf }}-\mathrm{P}_{\text {sat }}\left(\mathrm{T}_{\mathrm{o}}\right)\right] / \alpha \mathrm{H}_{\mathrm{o}}$ represents the difference between the pool surface pressure and the vapor pressure of the liquid at temperature $T_{0}$, divided by pressure increase at the depth of gas release. Evaluation is shown in Figure 3-2, assuming $\alpha=0.41 \mathrm{psi} / \mathrm{ft}$ and $\mathrm{H}_{\mathrm{o}}=26 \mathrm{ft}$. Figure 3-2 illustrates that tank bump events are doubtful if the pool surface temperature is just $10^{\circ} \mathrm{C}$ below the boiling point. This expression also demonstrates that in the limit of a shallow pool $(\beta \rightarrow \infty), \mathrm{V} / \mathrm{V}_{\mathrm{o}} \rightarrow 1$. In other words, there is no expansion for a shallow pool and a tank bump cannot occur.

\subsubsection{Tank 241-C-106 Steam Bump Evaluation, (Epstein et al. 1999)}

In this document, Epstein et al. (1999) give numerical examples to show that tank bumps cannot occur for shallow pools. If liquid temperature is $99^{\circ} \mathrm{C}\left(\mathrm{P}_{\text {sat }}=97,780 \mathrm{~Pa}\right)$ and pool depth is $1.0 \mathrm{~m}$, $\mathrm{V} / \mathrm{V}_{\mathrm{o}}$ is 3.7 at most, assuming the pool surface pressure is one atmosphere. If the pool depth is increased to $10.0 \mathrm{~m}$ but other assumptions remain unchanged, the maximum expansion ratio is 28. If liquid temperature is $95^{\circ} \mathrm{C}\left(\mathrm{P}_{\text {sat }}=84,550 \mathrm{~Pa}\right)$ and pool depth is $1.0 \mathrm{~m}$, maximum expansion ratio is 1.6, assuming other assumptions remain unchanged. A pool depth of $1.0 \mathrm{~m}$ is most relevant because the 241-C-106 waste will be sluiced to this depth, more or less. In regard to tank 241-C-106, tank bumps by inert gas injection are impossible because the supernatant is only a few inches deep.

\subsubsection{GOTH Analyses (Sathyanarayana 1996)}

The purpose of the WHC calculations using GOTH was to provide a technical basis for tank bumps and estimates of the consequences (releases of steam and solid particles) of tank bump events. The document begins by stressing that although current tank conditions preclude a tank bump event if normal ventilation is operating, loss of ventilation combined with increased nonconvective layer thicknesses and heat load might lead to favorable conditions for a bump. Without dome ventilation, local non-convective layer temperature can increase to saturation temperature. Two types of non-convective layer vertical temperature distributions are envisioned by the report: "best-estimate" and "conservative". A best-estimate temperature distribution is defined as the vertical temperature distribution just when the first piece of nonconvective waste reaches the local saturation temperature. This temperature distribution is said 
Figure 3-2. Cavity Expansion During Rise in a Constant Temperature Pool.

TYPICAL DEPTH BELOW SURFACE, Ft.

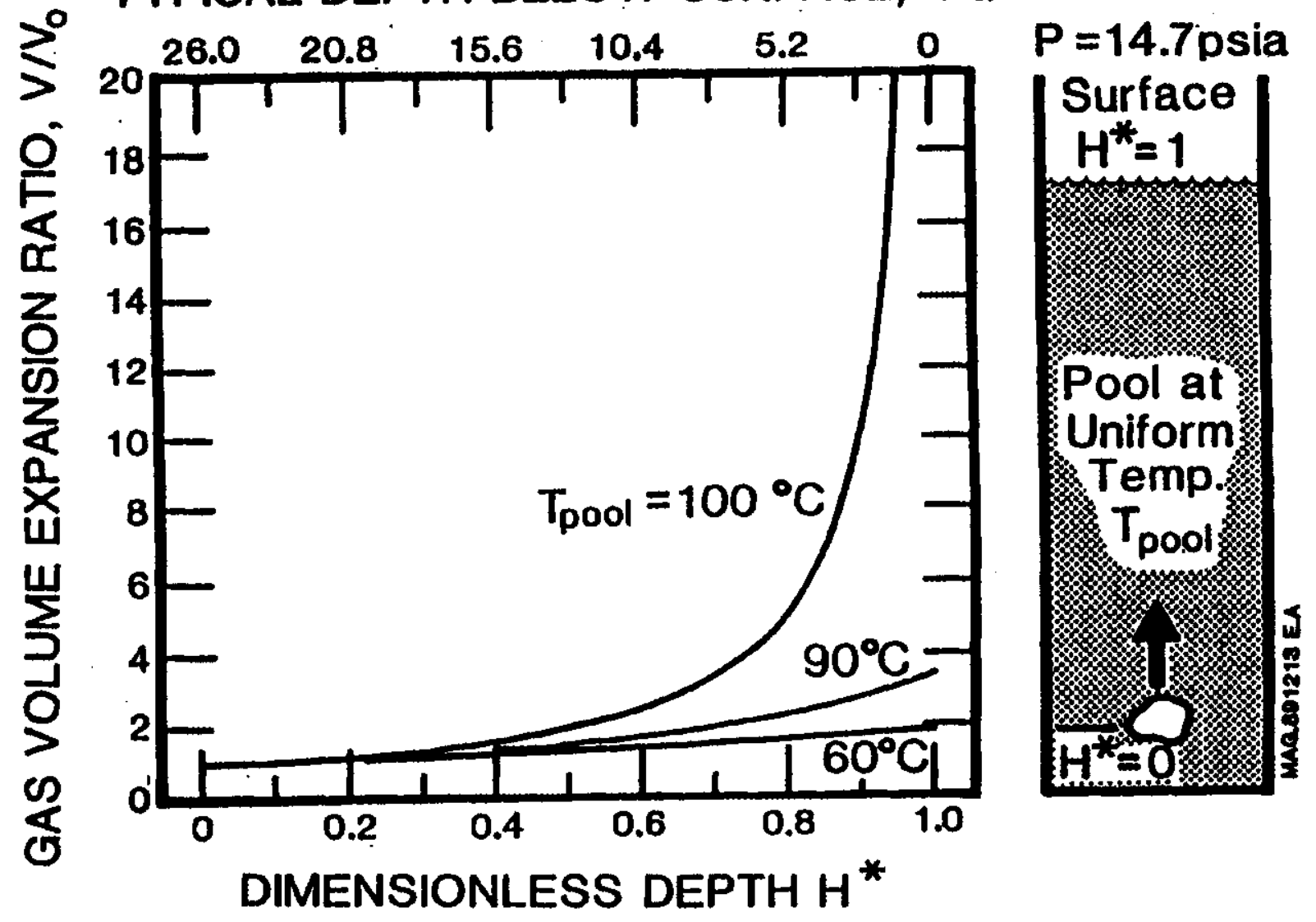


to exist for the first plume release only. Repeated bumps are expected because without dome ventilation the heat load continues. After the first bump, hot non-convective waste mixes with cooler waste until it is everywhere near the local saturation temperature; this is referred to as the conservative temperature distribution.

Sathyanarayana (1996) lists the following parameters and assumptions as critical to the magnitude of the bump and mass of airborne aerosol:

- Thickness: larger non-convective layer thicknesses produce larger bumps and releases. GOTH analyses assumed three thicknesses: 18 and 48 inch thicknesses with tank 241-AZ-101 solid and liquid waste properties (see Table 3-2) for each, and a thickness of $11 \mathrm{ft}$ with composite properties of tank 241-C-106 and 241-AY-102 wastes (see Table 3-2).

- Initial Temperature Distribution: the two assumed distributions - bestestimate and conservative - have been described above. The strength of the bump is proportional to the fraction of waste that is near the saturation temperature.

- Ventilation and Flow Paths: simulations were performed with three assumed flow areas, as shown in Table 3-3. In addition, preliminary calculations were made using the $\mathrm{AZ}$ tank farm 702-A ventilation system.

- Aerosol Deposition: the only deposition in the system is settling in the tank waste following release.

- Supernatant Depth: deeper pools result in higher tank bottom pressures and correspondingly higher saturation temperatures, which means that the bump will be more energetic when it finally happens. No attempt was made to study the effect pool depth. The total waste depth was $30 \mathrm{ft}$ for all simulations.

- Non-Convective Layer Viscosity: $8,000 \mathrm{lbm} / \mathrm{ft}-\mathrm{s}(12,000 \mathrm{~kg} / \mathrm{m}-\mathrm{s})$ was assumed for all cases. Viscosity partially determines the ability to trap steam bubbles.

- Non-Convective Waste Yield Strength: the assumption is that yield strength is zero, for the sake of conservatism. High yield strength would hold some waste in place and prevent it from participating in the rollover.

- Supernatant Temperature: pool sub-cooling has a dramatic effect on the strength of a bump. Various sub-cooled temperatures were considered.

Thermal hydraulic simulations were performed with the GOTH code.

Initial conditions were generated by a set of one-dimensional GOTH runs to find the vertical temperature distribution in the waste when the peak temperature (at the bottom) exceeds the local saturation temperature. Prior to GOTH simulation for a bump, a preliminary run was needed to 
Table 3-2. Waste Properties for GOTH Analysis (Sathyanarayana 1996).

\begin{tabular}{|c|c|c|}
\hline & $241-A Z-101$ & $\begin{array}{c}\text { 241-AY-102 } \\
\text { / 241-C-1066 }\end{array}$ \\
\hline \multicolumn{3}{|l|}{ Tank } \\
\hline Primary Tank Dia., ft. (m) & $75(22.9)$ & $75(22.9)$ \\
\hline Secondary Tank Dia., ft. (m) & $80(24.4)$ & $80(24.4)$ \\
\hline \multicolumn{3}{|l|}{ Trank Contents } \\
\hline Solids Density, $\mathrm{lbm} / \mathrm{ft}^{3}\left(\mathrm{~kg} / \mathrm{m}^{3}\right)$ & $243.4(3898.9)$ & $113.9(1822.4)$ \\
\hline Aqueous Solution Density, $\mathrm{lbm} / \mathrm{ft}^{3}\left(\mathrm{~kg} / \mathrm{m}^{3}\right)$ & $75.5(1209.4)$ & $75.5(1209.4)$ \\
\hline Sludge (Mixture) Density, $1 \mathrm{bm} / \mathrm{ft}^{3}\left(\mathrm{~kg} / \mathrm{m}^{3}\right)$ & $104.0(1665.9)$ & $81.2(1299.2)$ \\
\hline Total Waste Depth, ft. (m) & $30 \quad(9.14)$ & $30(9.14)$ \\
\hline Supernatant Depth, ft. (m) & $28.5 / 26 \quad(8.7 / 7.9)$ & $19(5.8)$ \\
\hline Sludge Depth, ft. (m) & $1.5 / 4 \quad(0.46 / 1.22)$ & $11(3.35)$ \\
\hline AY-102 Heat Load, Btu/hr (W) & & $33,000 \quad(9671)$ \\
\hline C-106 Sludge Heat Load, Btu/hr (W) & & $92,400(27080)$ \\
\hline Total Heat Load, Btu/hr (W) & & $125,000 \quad(36634)$ \\
\hline \multicolumn{3}{|l|}{ Supernatant } \\
\hline Heat Capacity, Btu/lbm-F (J/kg-K) & $0.8(3349)$ & $0.8(3349)$ \\
\hline Thermal Conductivity, Btu/hr-ft-F (W/m-K) & $0.35 \quad(0.61)$ & $0.35 \quad(0.61)$ \\
\hline Heat Generation Rate, Btu/hr-lbm (J/s-kg) & $0.019 \quad(0.0123)$ & $0.0(0.0)$ \\
\hline \multicolumn{3}{|l|}{ Insoluble Solids: } \\
\hline Heat Capacity, Btu/lbm-F (J/kg-K) & $0.2(837)$ & $0.2(837)$ \\
\hline Thermal Conductivity, Btu/hr-ft-F (W/m-K) & $5.0(8.5)$ & $5.0(8.5)$ \\
\hline Heat Generation, unwashed, Btu/hr-lbm (J/s-kg) & $0.37 \quad(0.2391)$ & $0.14(0.09)$ \\
\hline Volume Fraction of Solids, unwashed, $\%$ & 17 & 16 \\
\hline Particle Size, washed & $\begin{array}{c}1-10 \text { microns, } \\
5 \text { micron average }\end{array}$ & $\begin{array}{c}1-10 \text { microns, } \\
5 \text { micron average }\end{array}$ \\
\hline
\end{tabular}

Table 3-3. GOTH Runs Flow Path Descriptions.

\begin{tabular}{|l|l|l|}
\hline \multicolumn{4}{|c|}{ Dome Flow Path Assumptions } \\
\hline Riser Flanges, Cover Block Open & Five 4" Dia. Inleakage Paths & One 6" Dia. Inleakage \\
\hline 1. 42" dia. Pump Pit Path & Five 4" dia. Inleakage Paths & 6" dia. Inleakage Path \\
\hline 2. $20^{\prime \prime}$ dia. Vent Path & $20^{\prime \prime}$ dia. Vent Path & $20^{\prime \prime}$ dia. Vent Path \\
\hline 3. Four 42" dia. Sluice Pits Path & & \\
\hline
\end{tabular}


find conditions that would initiate the bump. These preliminary runs simulated the transient heat up of the waste assuming normal ventilation is replaced by an air inlet flow at $53.34^{\circ} \mathrm{F}$, which is the Hanford annual average temperature. For example, a GOTH calculation resulted in the bestestimate initial conditions for $11 \mathrm{ft}$ of consolidated non-convective layer from tanks 241-AY-102 and 241-C-106, as shown in Figure 3-3. The conservative temperature profile shown in Figure 3-4 results from assuming that at each point, the waste temperature corresponds to saturation. These calculations were repeated for the 18 and 48 inch depths, although only the $11 \mathrm{ft}$ temperature profile is shown here because the temperature distribution is more apparent.

The results of a GOTH run for the 18 -inch non-convective layer with no condensation are shown in Figure 3-5, which represents the development of steam concentration contours in the waste. The figure shows gas volume fraction contour snapshots at four different times. Starting with the top left-hand corner, the frames show the start of the steam bump and development of hydrodynamic instability in the waste. In this figure, the maximum void fraction plotted is 0.9 and the minimum is 0.1 . The top left-hand frame shows that 40 seconds into the run, the void fraction at the height of the original non-convective layer is about 0.1 . Hydrodynamic instabilities start about five seconds later, as shown by the top right-hand frame, with a superheated plume identifiable at 50 seconds. Contours at 70 seconds show a plume breaking the waste surface and dispersing liquid droplets and solid particles to the dome space. Although not shown here, Sathyanarayana (1996) plots both liquid droplet and solid particle flowrate through the respective tank vent paths as a function of time. Results are also reported as integrated, total releases of liquid and particle mass. The report also goes on to state that bumps will repeat until ventilation is restored.

The matrix of GOTH runs and a brief description of results are shown in Table 3-4. Important results culled from the table are as follows:

- For the conservative temperature profile with no condensation, dome pressurization is significant and particle releases can be enormous. Release quantity depends on the size and location of flow paths, but as a general trend releases are largest for "Riser Flanges, Cover Blocks Open" cases, which have the largest flow area. Releases for "One 6" Dia. Inleakage Path" cases are much smaller, although still significant. GOTH calculations are multi-dimensional, which means that the location of the bump with respect to the openings is important for determining releases.

- For 18 and 48-inch non-convective thicknesses, best-estimate cases do not result in bumps, regardless of assumptions about condensation.

- For the $11 \mathrm{ft}$ thickness, no bump occurs in the best-estimate case with condensation.

- $\quad$ Comparing Run 13 (peak pressure $=25.0$ psia, release $=2100 \mathrm{lbm}$ ) with Run 22 (peak pressure $=22.2$ psia, release $=450 \mathrm{lbm}$ ) shows that a simple model provides a conservative estimate with respect to a complete ventilation model that considers all four tanks in the farm and other ventilation equipment. 
Figure 3-3. Best-Estimate Initial Temperature Profile for $11 \mathrm{ft}$. of Consolidated Non-Convective Waste.

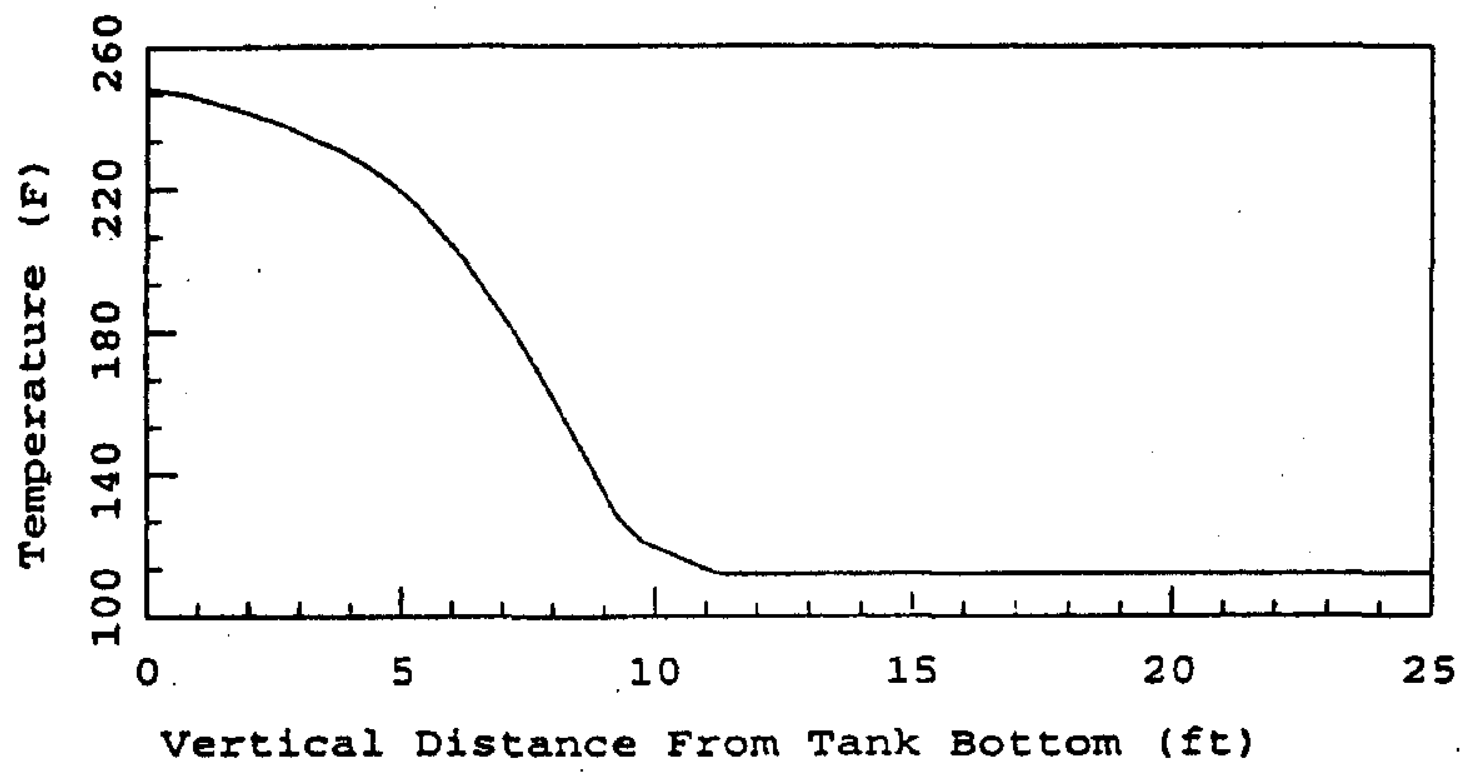


Figure 3-4. Conservative Initial Temperature Profile for $11 \mathrm{ft}$. of Consolidated Non-Convective Waste.

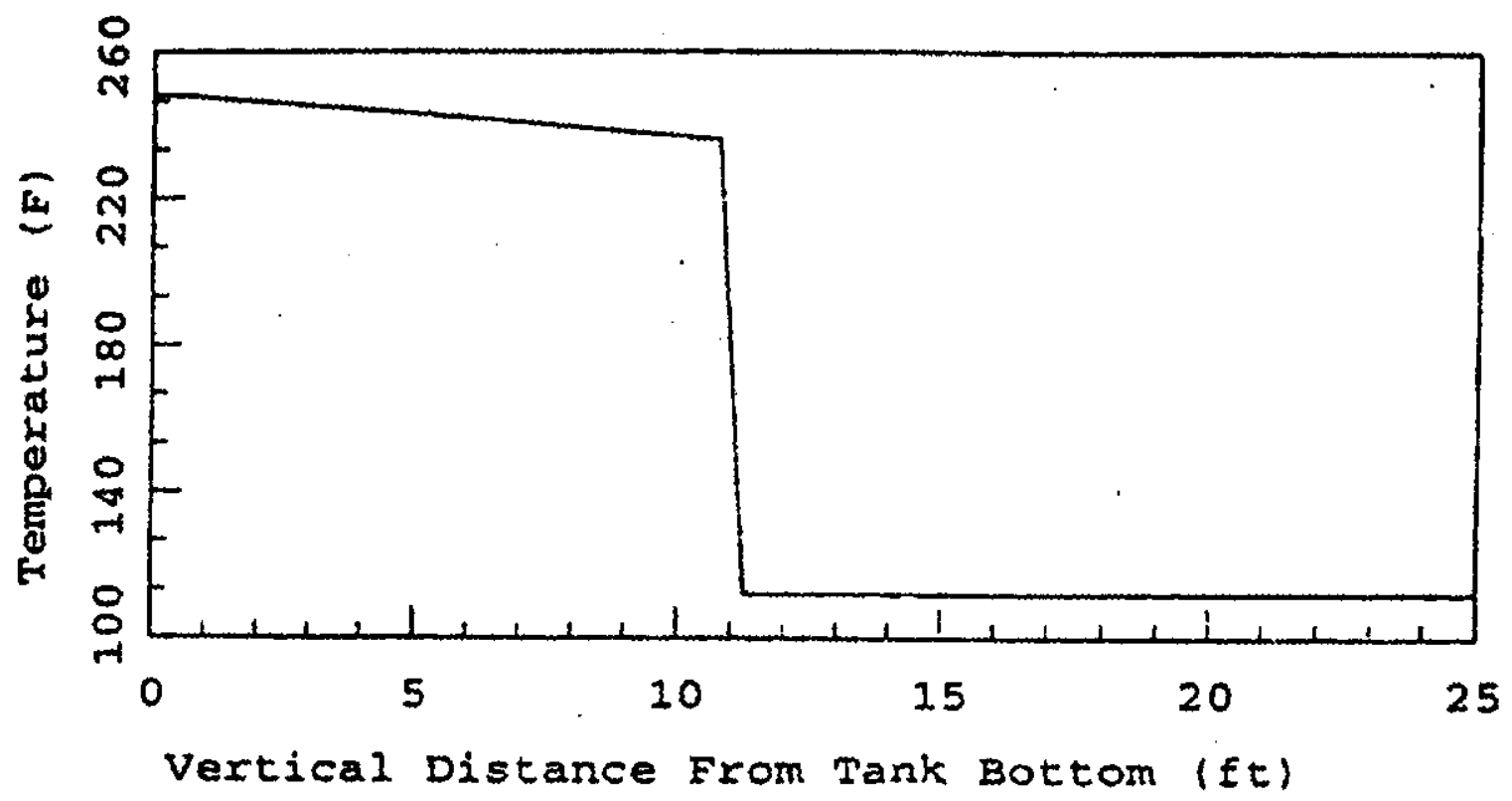


Figure 3-5. Gas Volume Fraction Contours for GOTH Run With 18" Non-Convective Layer and No Condensation.

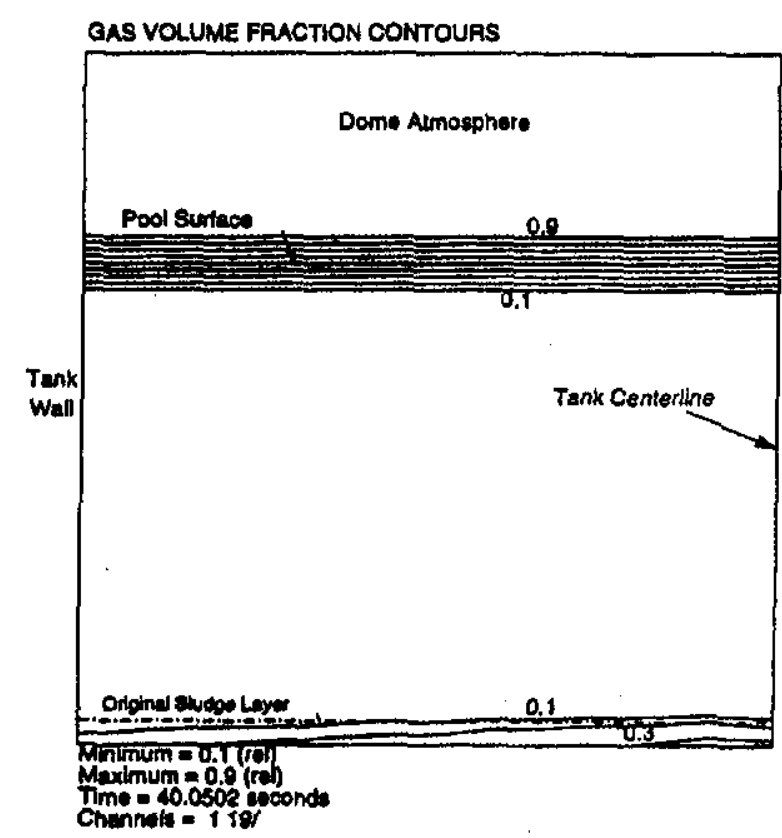

GAS VOLUME FAACTION CONTOUAS
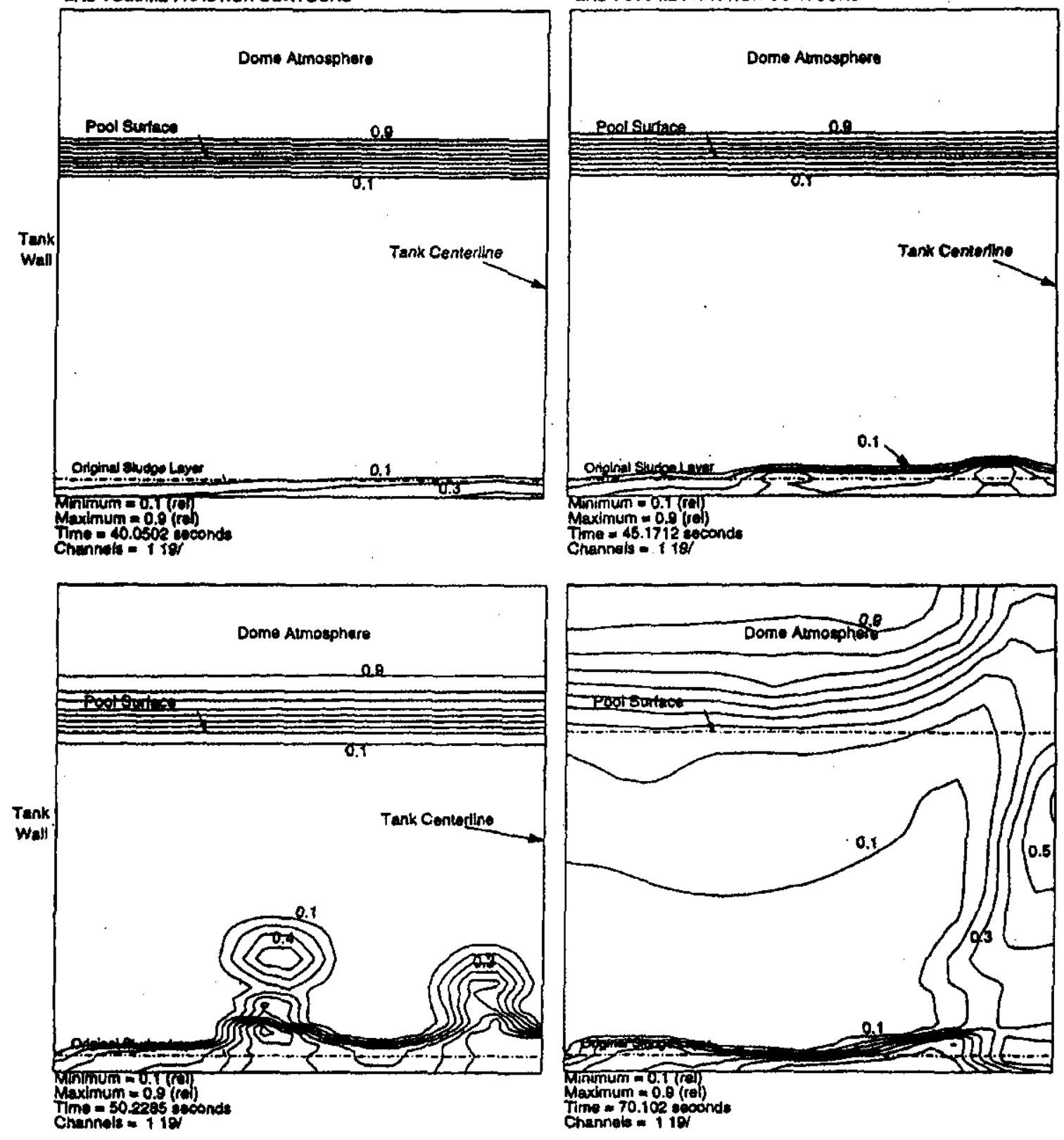

Thme = 46.1712 gecond

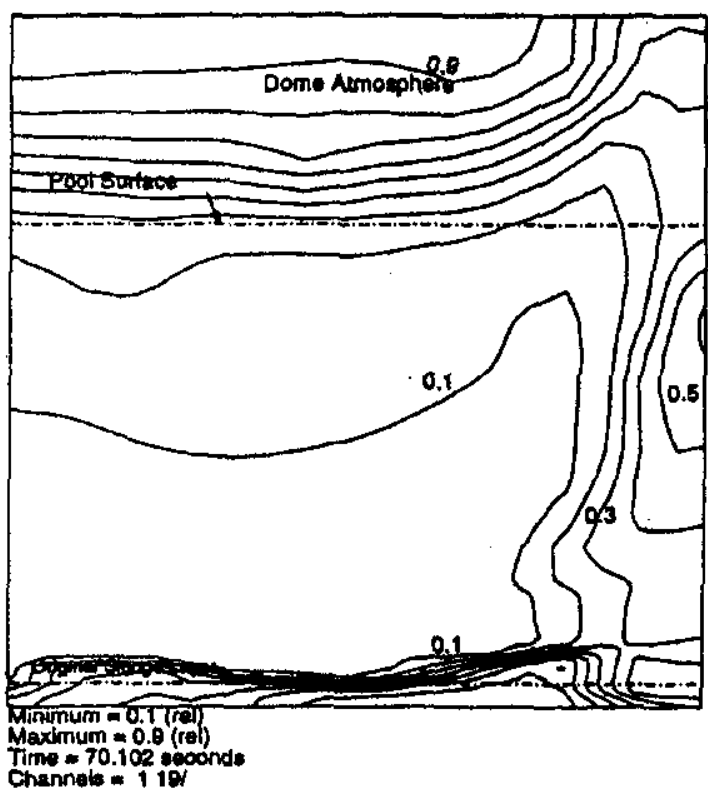


Table 3-4. GOTH Run Summary.

\begin{tabular}{|c|c|c|c|c|c|c|c|}
\hline Run & $\begin{array}{l}\text { Sludge } \\
\text { Depth } \\
\text { (ft) }\end{array}$ & $\begin{array}{c}\text { Waste } \\
\text { Properties }\end{array}$ & $\begin{array}{c}\text { Temperature } \\
\text { I.C. }\end{array}$ & $\begin{array}{l}\text { Conden- } \\
\text { sation }\end{array}$ & Flow Area ${ }^{(1)}$ & $\begin{array}{c}\text { Peak Dome } \\
\text { Pressure } \\
\text { (psia) }\end{array}$ & $\begin{array}{c}\text { Particle } \\
\text { Release } \\
\text { (lbm) }\end{array}$ \\
\hline 1 & 1.5 & $241-A Z-101$ & Conservative & No & $\begin{array}{c}\text { Riser Flanges, } \\
\text { Cover Blocks Open }\end{array}$ & 15.0 & 150 \\
\hline 2 & 1.5 & $241-A Z-101$ & Conservative & No & $\begin{array}{c}\text { Five 4" Dia. } \\
\text { Inleakage Paths }\end{array}$ & 15.7 & 1.3 \\
\hline 3 & 1.5 & $241-A Z-101$ & Conservative & No & $\begin{array}{c}\text { One 6" Dia. } \\
\text { Inleakage Path }\end{array}$ & 15.5 & 7 \\
\hline 4 & 1.5 & 241-AZ-101 & Conservative & Yes & No bump & No bump & $\begin{array}{c}\text { No } \\
\text { bump }\end{array}$ \\
\hline 5 & 1.5 & 241-AZ-101 & Best-Estimate & No & $\begin{array}{c}\text { Riser Flanges, } \\
\text { Cover Blocks Open }\end{array}$ & No bump & $\begin{array}{c}\text { No } \\
\text { bump }\end{array}$ \\
\hline 6 & 4 & 241-AZ-101 & Conservative & No & $\begin{array}{c}\text { Riser Flanges, } \\
\text { Cover Blocks Open }\end{array}$ & 22.5 & 6,300 \\
\hline 7 & 4 & $241-A Z-101$ & Conservative & No & $\begin{array}{c}\text { Five 4" Dia. } \\
\text { Inleakage Paths }\end{array}$ & 17.3 & 85 \\
\hline 8 & 4 & 241-AZ-101 & Conservative & No & $\begin{array}{c}\text { One 6" Dia. } \\
\text { Inleakage Path }\end{array}$ & 19.5 & 165 \\
\hline 9 & 4 & 241-AZ-101 & Conservative & $\begin{array}{l}\text { Yes }-164^{\circ} \mathrm{F} \\
\text { Supernatant }\end{array}$ & & & \\
\hline 10 & 4 & 241-AZ-101 & Best-Estimate & No & $\begin{array}{c}\text { Riser Flanges, } \\
\text { Cover Blocks Open }\end{array}$ & - & Minimal \\
\hline 11 & 4 & 241-AZ-101 & Best-Estimate & Yes & $\begin{array}{c}\text { Riser Flanges, } \\
\text { Cover Blocks Open }\end{array}$ & No bump & $\begin{array}{c}\text { No } \\
\text { bump }\end{array}$ \\
\hline 12 & 11 & $\begin{array}{l}241-\mathrm{C}-106 / \\
241-\mathrm{AY}-102\end{array}$ & Conservative & No & $\begin{array}{c}\text { Riser Flanges, } \\
\text { Cover Blocks Open }\end{array}$ & 27.0 & 97,000 \\
\hline 13 & 11 & $\begin{array}{l}241-\mathrm{C}-106 / \\
241-\mathrm{AY}-102 \\
\end{array}$ & Conservative & No & $\begin{array}{c}\text { Five 4" Dia. } \\
\text { Inleakage Paths }\end{array}$ & 25.0 & 2,100 \\
\hline 14 & 11 & $\begin{array}{l}241-\mathrm{C}-106 / \\
241-\mathrm{AY}-102\end{array}$ & Conservative & No & $\begin{array}{c}\text { One 6" Dia. } \\
\text { Inleakage Path }\end{array}$ & 25.0 & 1,050 \\
\hline 15 & 11 & $\begin{array}{l}241-\mathrm{C}-106 / \\
241-\mathrm{AY}-102 \\
\end{array}$ & Conservative & Yes & $\begin{array}{c}\text { Riser Flanges, } \\
\text { Cover Blocks Open }\end{array}$ & 28.0 & 6,200 \\
\hline 16 & 11 & $\begin{array}{l}241-C-106 / \\
241-A Y-102 \\
\end{array}$ & Conservative & Yes & $\begin{array}{c}\text { Five 4" Dia. } \\
\text { Inleakage Paths }\end{array}$ & 30.0 & 260 \\
\hline 17 & 11 & $\begin{array}{l}241-\mathrm{C}-106 / \\
241-\mathrm{AY}-102\end{array}$ & Conservative & Yes & $\begin{array}{c}\text { One 6" Dia. } \\
\text { Inleakage Path }\end{array}$ & 32.0 & 2.5 \\
\hline 18 & 11 & $\begin{array}{l}241-\mathrm{C}-106 / \\
241-\mathrm{AY}-102\end{array}$ & Best-Estimate & No & $\begin{array}{c}\text { Riser Flanges, } \\
\text { Cover Blocks Open }\end{array}$ & 18.8 & 90 \\
\hline 19 & 11 & $\begin{array}{l}241-\mathrm{C}-106 / \\
241-\mathrm{AY}-102 \\
\end{array}$ & Best-Estimate & No & $\begin{array}{c}\text { Five 4" Dia. } \\
\text { Inleakage Paths }\end{array}$ & 19.2 & 78 \\
\hline 20 & 11 & $\begin{array}{l}241-\mathrm{C}-106 / \\
241-\mathrm{AY}-102\end{array}$ & Best-Estimate & No & $\begin{array}{c}\text { One } 6 " \text { Dia. } \\
\text { Inleakage Path }\end{array}$ & 19.7 & 32 \\
\hline 21 & 11 & $\begin{array}{l}241-\mathrm{C}-106 / \\
241-\mathrm{AY}-102\end{array}$ & Best-Estimate & $\begin{array}{l}\text { Yes }-112^{\circ} \mathrm{F} \\
\text { Supernatant }\end{array}$ & $\begin{array}{c}\text { Riser Flanges, } \\
\text { Cover Blocks Open }\end{array}$ & No bump & $\begin{array}{l}\text { No } \\
\text { bump }\end{array}$ \\
\hline 22 & 11 & $241-A Z-101$ & Conservative & No & $\begin{array}{l}\text { AZ/AY Vent Farm } \\
\text { Model }\end{array}$ & 22.2 & 450 \\
\hline
\end{tabular}


In cases where bumps occur, the severity of predicted releases can be traced to the conservative assumptions made for the analyses. Total waste depth is $30 \mathrm{ft}$. in all cases, which means that the small dome free volume cannot accommodate a large bump and that liquid slugs or droplets are pushed out dome flow paths. With a non-convective waste shear yield strength of zero, all of the waste participates in the bump event, which means that the void created is much larger than if only a small portion participated.

\subsubsection{Tank Bump Potential During In-Tank Washing Operations Proposed for the AZ Tanks (Waters et al. 1991)}

Waters et al. (1991) prepared this work to support "in-tank sludge washing" in DSTs 241-AZ-101 and -102. The report identified the physical conditions needed for a bump as follows:

1. Supernatant temperature must be near the boiling point,

2. There must be a heat generating non-convective layer,

3. Non-convective layer temperature must exceed saturation at the local hydrostatic pressure,

4. Radiolytic heat load must be greater than $300 \mathrm{~kW}(1 \mathrm{Mbtu} / \mathrm{hr})$,

5. Steaming rate must exceed the capacity of the vent line, which is about $4.43 \mathrm{~m}^{3} / \mathrm{s}\left(9400 \mathrm{ft}^{3} / \mathrm{min}\right)$, and

6. Superheat with respect to the supernatant atmosphere boiling point must be $2.1 \times 10^{10} \mathrm{~J}(20 \mathrm{Mbtu})$.

The first three items are essentially the same as the conclusions in the FAI report of 1991, while the remaining three items are based on historical tank bump events. At the time of the report, tank 241-AZ-101 heat generation rate was projected to be $83.2 \mathrm{~kW}$ for April of 1993 and $73 \mathrm{~kW}$ for October of 1997.

Motivation for the report was the in-tank sludge washing procedure that used four mixer pumps dissipating a total of $800 \mathrm{~kW}\left(2.73 \times 10^{6} \mathrm{Btu} / \mathrm{hr}\right.$ or $\left.1073 \mathrm{hp}\right)$. Waters et al. (1991) used HEATING7 (Childs 1991) to determine if normal and off-normal sludge washing conditions lead to tank bump conditions. The first simulation performed was to determine if the waste temperatures will remain below saturated conditions under normal ventilation and prolonged input of $800 \mathrm{~kW}$. Subsequent runs postulated power outages of various duration that begin after prolonged mixer pump operation has increased waste temperatures. And finally, a run was made with initial conditions prior to mixing and a 100-day outage of the airlift circulators and normal ventilation systems.

The HEATING7 model for transient thermal analysis in the three-dimensional cylindrical coordinate system is shown here as Figure 3-6. Six boundary conditions are required. The first (BCl in Figure 3-6) is forced convection from the soil to the Hanford environment, assuming a 
Figure 3-6. Dimensional HEATING7 Model (Waters et al. 1991).

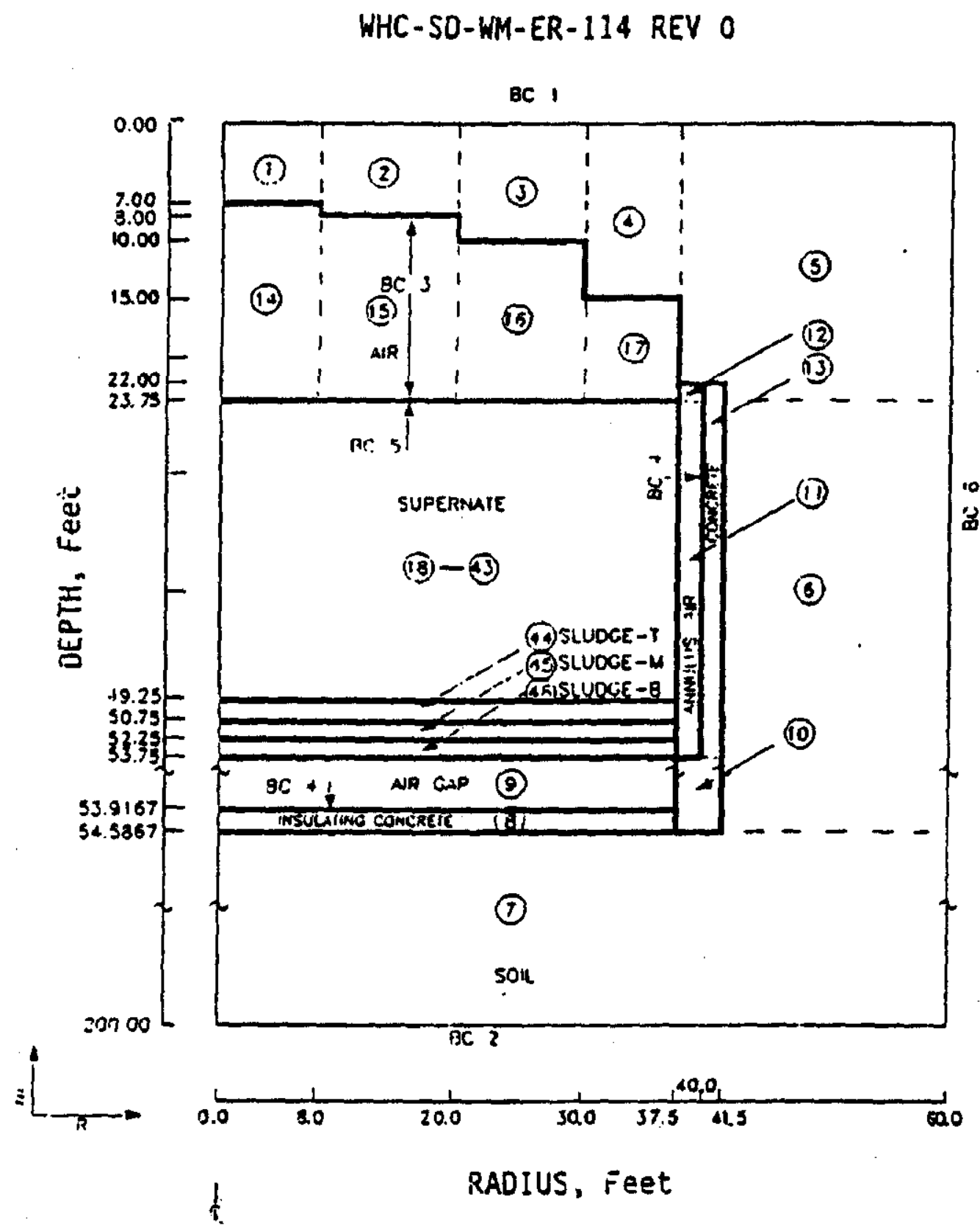

GIPR ILO RIIMGERS KF.PRE,SENT HEATINCT REGION NUMBERS 
heat transfer coefficient of $11.4 \mathrm{~W} / \mathrm{m}^{2}-\mathrm{K}$ and average site temperature of $12.8^{\circ} \mathrm{C}\left(55^{\circ} \mathrm{F}\right)$. The second boundary condition (BC2) is a constant temperature of $12.8^{\circ} \mathrm{C}$ at the water table. Boundary condition 3 represents the radiative heat transfer and natural convection processes at the supernatant-dome interface. Boundary condition 4 approximates heat removal from the steel primary tank by the annulus ventilation system. Boundary condition 5 incorporates evaporative cooling as explained below, and heat transfer to bubbles rising through the supernatant by way of ALC operation. Boundary condition 6 is an adiabatic condition to simulate an array of tanks with a 120 -ft center-to-center spacing.

Evaporative cooling was included in the model based on the following relationship from Perry (1950):

$$
\mathrm{W}=0.0138\left(\mathrm{P}_{\mathrm{svp}}-\mathrm{P}_{\mathrm{p}}\right)^{1.20}
$$

where $\mathrm{W}$ is the evaporation rate in $\mathrm{lb} / \mathrm{hr}-\mathrm{ft}^{2}, \mathrm{P}_{\text {svp }}$ is the vapor pressure of the waste liquid at the surface in $\mathrm{mm} \mathrm{Hg}$, and $\mathrm{P}_{\mathrm{p}}$ is the partial pressure of water vapor in the airspace above the surface. Waters et al (1991) was able to reconcile this equation with the results shown in Figure 3-1. Flow rates were $634 \mathrm{scfm}$ for normal primary ventilation and $500 \mathrm{scfm}$ for annulus flow, with inlet temperature and relative humidity of $70^{\circ} \mathrm{F}$ and $50 \%$, respectively. Airlift circulators flow rate was $154 \mathrm{scfm}$ before and during mixer operation. In outage scenarios, all flow rates were set to zero.

Results of the HEATING7 runs show the following:

1. Primary ventilation controls the equilibrium waste temperature by evaporative cooling,

2. Given ventilation system operation, waste temperatures remain below saturation, even with prolonged $800 \mathrm{~kW}$ power input,

3. Bump conditions do not result from a $72 \mathrm{hr}$ power outage that begins after mixer pump operation has increased waste temperature,

4. Bump conditions do not arise after a 100 day outage that begins prior to mixer pump operation, and

5. A permanent outage could result in tank bump conditions under certain circumstances.

Waters et al. (1991) did not specify what circumstances lead to tank bump conditions.

\subsection{CHARACTERIZATION DATA}

The brief survey above suggests that the following waste parameters are needed for analysis of tank bump potential: non-convective layer depth, supernatant depth, and total waste volume. Tank-specific values for these parameters, along with many of the basic inputs that will be 
needed in the calculations to follow, are found in $\mathrm{Hu}$ et al. (2000) and Barton and Bingham (1999).

The analyses surveyed in the previous section show that only tanks with a deep supernatant layer are relevant. SSTs with appreciable amounts of liquid are listed in Table 3-5; a value of $400 \mathrm{~kL}$ (about $110 \mathrm{kgal}$ ) is the criterion here because this amount of liquid would result in a $1.0 \mathrm{~m}$ deep supernatant layer, assuming that the waste in these tanks is separated into distinct non-convective and supernatant layers. Core samples suggest that for the SSTs listed here, this is not really the case. For example, 241-A-101 and 241-AX-101 were found to have solids floating on the free liquid (Stewart et al. 1996). These SSTs are nevertheless considered in Section 6.0, where tank inventories and heat loads will be compared against bump criteria to develop a short list of tank that are susceptible to bumps. DST supernatant depths are generally significant for tank bump phenomenology. Supernatant layer depths range from $20 \mathrm{~cm}$ to $10.3 \mathrm{~m}$, and non-convective layer depths range from $0 \mathrm{~m}$ to $5.4 \mathrm{~m}$. A few DSTs have deep supernatant layers but trivial nonconvective layer depths; e.g., 241-AP-102 has $10 \mathrm{~m}$ of supernatant but no non-convective waste. Parts of Hu et al. (2000) are included here as Appendix A. In Section 6.0, supernatant and nonconvective layer depths will be compared against bump criteria to develop a list of tanks potentially susceptible to tank bumps.

Table 3-5. SSTs With Liquid Inventory $>400 \mathrm{~kL}$.

\begin{tabular}{|c|c|c|}
\hline Tank & Liquid Volume (kL) & Waste Volume (kL) \\
\hline $241-\mathrm{A}-101^{*}$ & 1923 & 3608 \\
\hline $214-\mathrm{AX}-101^{*}$ & 1461 & 2831 \\
\hline $214-\mathrm{S}-111^{*}$ & 420 & 2044 \\
\hline $241-\mathrm{SX}-102$ & 507 & 1946 \\
\hline
\end{tabular}

Waste properties are also key to evaluation of tank bump potential and consequences, in particular the thermophysical properties of heat capacity, viscosity, thermal conductivity, density, and heat generation rate. Thermophysical properties are highly uncertain, and vary from tank to tank, as well as at different locations within the same tank. The mechanical property of shear yield strength, also referred to as shear strength or simply yield strength; is an input to predictive models for the size of the "gob" released from the non-convective layer. A summary of the available property data is presented below.

Using an analysis of variance tool, Willingham (1994) compiled a statistical analysis of waste properties and listed the total variability of several key properties. Source data came from characterizations for individual tanks. Data were available from only 33 of the 177 tanks and were not grouped into SST and DST categories; i.e., the statistical summary includes data from both tank types to calculate mean, standard deviation, etc. Sources of variability include tank-totank variation, core-to-core (radial difference) variation, segment-to-segment (axial difference) variation, and measurement error. Willingham's (1994) compilation is presented here for completeness, but it is difficult to apply here because it does not distinguish between DSTs and SSTs, and has been obviated.

Since by and large only the double-shell tanks have significant supernatant depths, discussion can be narrowed to a survey of DST waste properties. Based on ball rheometer and void fraction 


\section{RPP-6213 REV 0}

instrument measurements taken between December 1994 and May 1996, Meyer et al. (1997) reported DST waste properties pertinent to gas retention and release behavior: density, yield stress, and viscosity. Meyer et al. (1997) list supernatant and non-convective densities based on data for five DSTs on the flammable tank watch list (241-SY-103, 241-AN-103, 241-AN-104, 214-AN-105, and 241-AW-101). These five salt cake tanks exhibit episodic gas releases that are typically less than $30 \mathrm{~m}^{3}$, although there have been episodes of releases as large as $100 \mathrm{~m}^{3}$ in 241-SY-101, the sixth tank on the list. Supernatant densities are in the range between $1430 \mathrm{~kg} / \mathrm{m}^{3}$ and $1600 \mathrm{~kg} / \mathrm{m}^{3}$, with an uncertainty of roughly $\pm 2 \%$ about any one value. Nonconvective layer densities are in the range $1570 \mathrm{~kg} / \mathrm{m}^{3}$ and $1730 \mathrm{~kg} / \mathrm{m}^{3}$, with an uncertainty of $\pm 6 \%$ or so about any one value.

Meyer et al. (1997) also list non-convective layer yield strength (referred to as shear strength by Willingham [1994]) as a function of depth for the same five tanks. The non-convective layer is a viscoplastic material with a finite yield strength and shear rate dependency that can be measured by a ball rheometer. Yield strength is largest near the bottom; maximum yield stress is somewhere between 200 and $325 \mathrm{~Pa}$, depending on the tank.

Another compilation of DST waste property data is given in Barton and Bingham (1999). Descriptive statistics were developed from this compilation, with the six salt cake DSTs differentiated from the other (sludge) DSTs. For the six salt cake DSTs, the mean and standard deviation of the convective layer density are 1.48 and $0.06 \mathrm{~g} / \mathrm{ml}$, respectively; mean and standard deviation of the non-convective layer density are 1.62 and $0.08 \mathrm{~g} / \mathrm{ml}$, respectively. For the remaining twenty-two DSTs, mean and standard deviation for the convective layer density are 1.13 and $0.12 \mathrm{~g} / \mathrm{ml}$, respectively. Mean and standard deviation for non-convective layer are 1.51 and $0.09 \mathrm{~g} / \mathrm{ml}$, respectively. Only fourteen DSTs were used for non-convective layer averaging because eight of the DSTs have only a trivial amount of settled solids. Waste yield strength is listed for many of the tanks, but in other instances a probability density function is used. This function is a truncated normal distribution with mean equal to $120 \mathrm{~Pa}$, standard deviation equal to $30 \mathrm{~Pa}$, minimum equal to $50 \mathrm{~Pa}$, and maximum equal to $200 \mathrm{~Pa}$. Tank-specific yield strengths listed range from 81 to $225 \mathrm{~Pa}$.

To conclude, Table 3-6 provides a summary of DST waste property data. Table 3-6 shows values obtained from averaging over the salt cake DSTs and sludge DSTs. Both the $\mathrm{Hu}$ et al. (2000) database and the pedigreed database were considered and found to be in reasonable agreement. The differences between values for the salt cake DSTs and values for the other DSTs should be noted. Mean non-convective layer and supernatant densities in the salt cake DSTs are higher, and the difference between non-convective layer density and supernatant density (e.g., 1.63 minus $1.50 \mathrm{~g} / \mathrm{ml}$ ) is smaller than the corresponding difference for a sludge DST (e.g., 1.51 minus $1.13 \mathrm{~g} / \mathrm{ml}$ ). The table therefore illustrates an important difference between salt cake and sludge tanks: the sludge tanks have a higher neutral buoyancy void fraction.

\subsection{TANK CONFIGURATION DATA}

SST and DST configurations are pictured here in Figures 3-7 and 3-8, respectively. Tank configuration data are important to consequences of the tank bump. These data include the headspace volume, headspace flowpaths, and decontamination factors for flowpaths from the 


\section{RPP-6213 REV 0}

Figure 3-7. Single-Shell Tank (SST) Instrumentation Configuration.

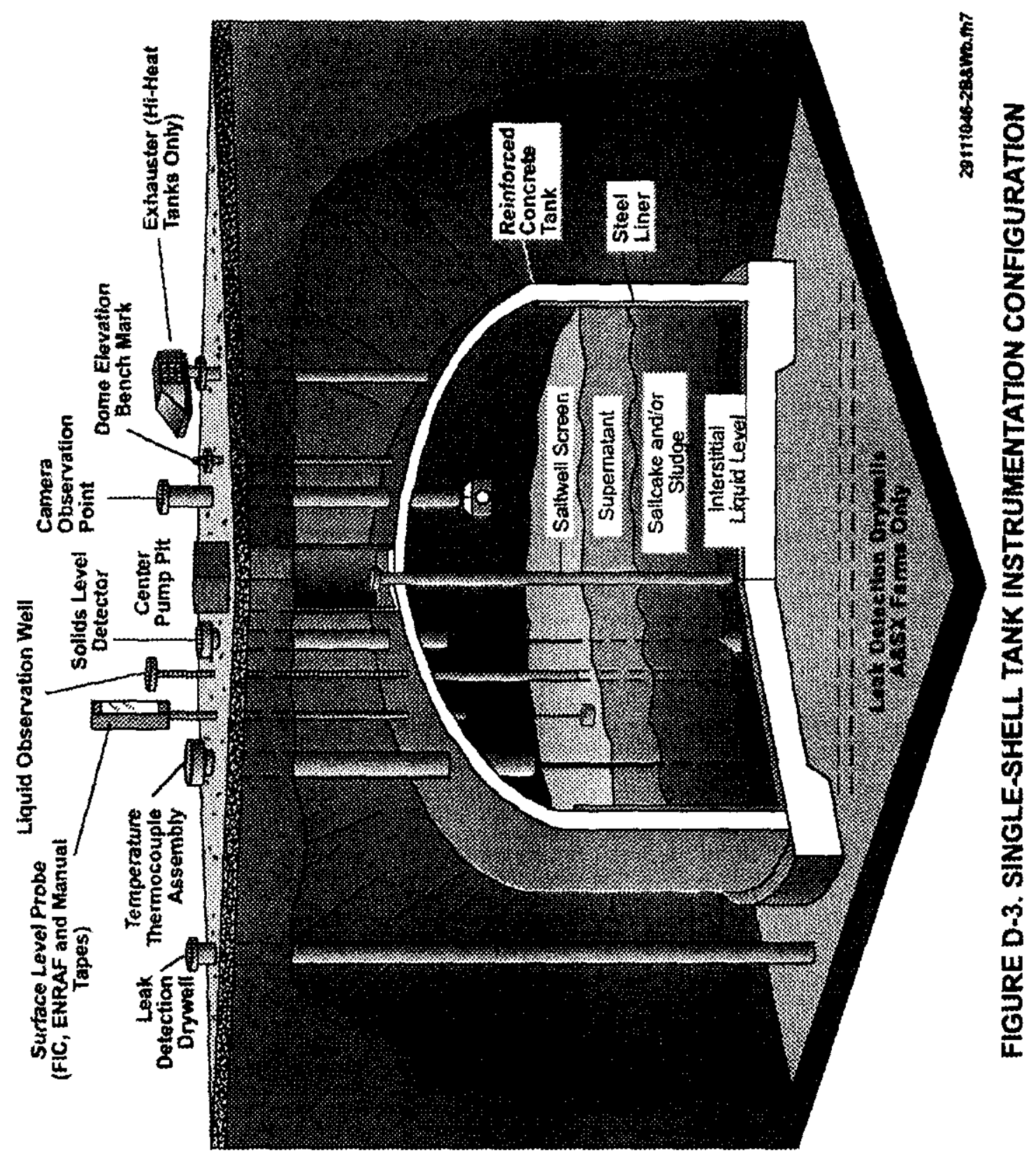


Figure 3-8. Double-Shell Tank (DST) Instrumentation Configuration.

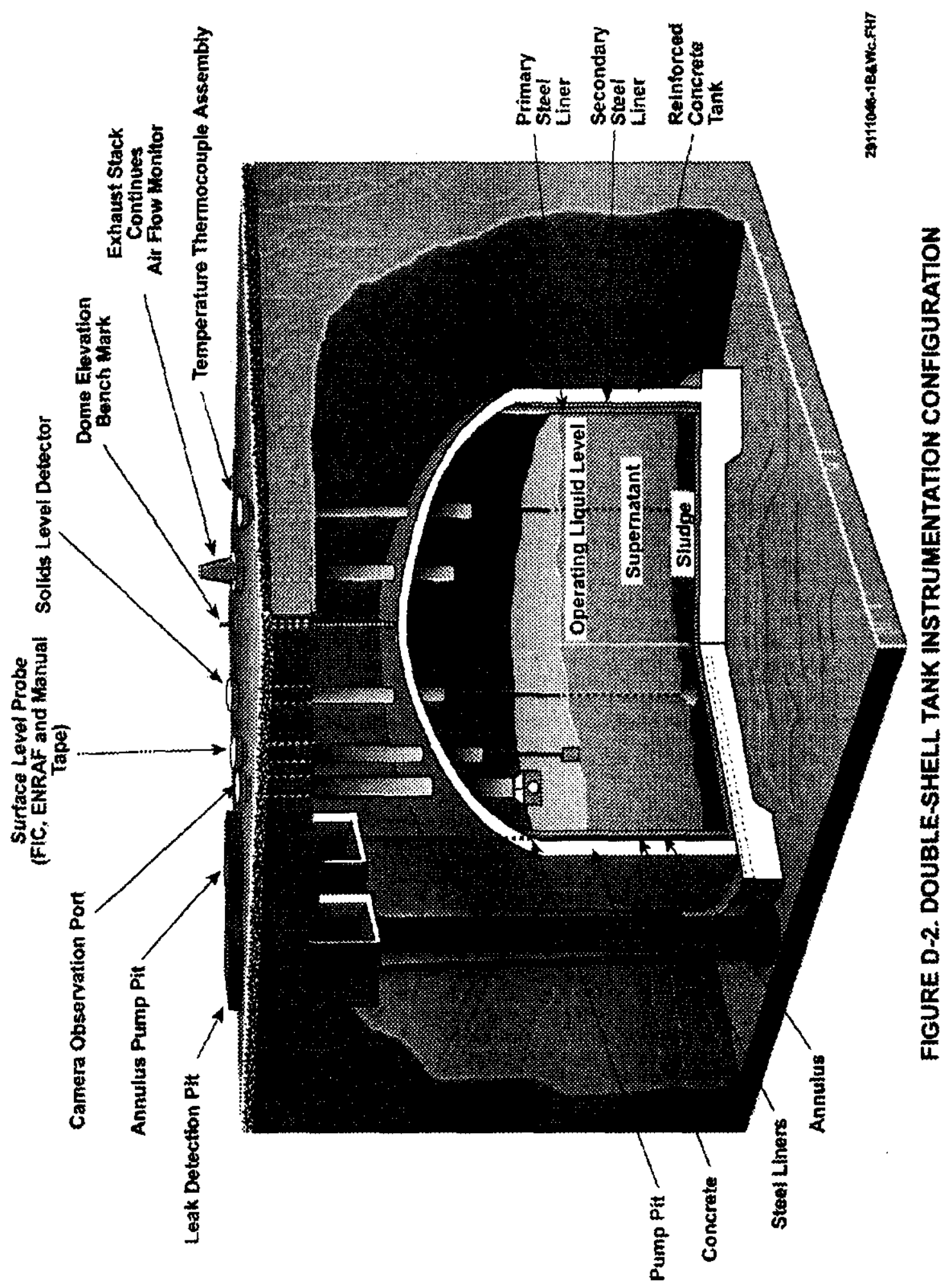


Table 3-6. DST Waste Properties.

\begin{tabular}{|c|c|c|c|c|}
\hline Property & Units & Source & Mean & Uncertainty \\
\hline \multirow{5}{*}{$\begin{array}{l}\text { Non- } \\
\text { Convective } \\
\text { Layer Density }\end{array}$} & \multirow{5}{*}{$\mathrm{g} / \mathrm{ml}$} & (Hu et al. 2000) Six Salt Cake DSTs & 160 & Stand. Dev. $=0.07$ \\
\hline & & (Meyer et al. 1997) Five Salt Cake DSTs & 163 & $\pm 6 \%$ \\
\hline & & $\begin{array}{c}\text { (Barton \& Bingham 1999) Six Salt Cake } \\
\text { DSTs }\end{array}$ & 162 & Stand. Dev. $=0.08$ \\
\hline & & $\begin{array}{c}\text { (Barton \& Bingham 1999) Fourteen Sludge } \\
\text { DSTs }\end{array}$ & 1.51 & Stand. Dev. $=0.09$ \\
\hline & & (Hu et al. 2000) Fifteen Sludge DSTs & 1.47 & Stand. Dev. $=0.12$ \\
\hline \multirow{5}{*}{$\begin{array}{l}\text { Supernatant } \\
\text { Density }\end{array}$} & \multirow{5}{*}{$\mathrm{g} / \mathrm{ml}$} & (Hu et al. 2000) Six Salt Cake DSTs & 43 & Stand. Dev. $=0.04$ \\
\hline & & (Meyer et al. 1997) Five Salt Cake DSTs & 150 & $\pm 2 \%$ \\
\hline & & $\begin{array}{c}\text { (Barton \& Bingham 1999) Six Salt Cake } \\
\text { DSTs }\end{array}$ & 148 & Stand. Dev. $=0.06$ \\
\hline & & $\begin{array}{l}\text { (Barton \& Bingham 1999) Twenty-Two } \\
\text { Sludge DSTs }\end{array}$ & 1.13 & Stand. Dev. $=0.12$ \\
\hline & & (Hu et al 2000) Twenty-Two Sludge DSTs & 1.19 & Stand. Dev. $=0.12$ \\
\hline \multirow[b]{2}{*}{ Shear Strength } & \multirow[b]{2}{*}{$\mathrm{Pa}$} & (Meyer et al. 1997) Five Salt Cake DSTs & $\begin{array}{l}263 \\
\text { (Max. } \\
\text { Value) }\end{array}$ & \pm 62 \\
\hline & & (Barton \& Bingham 1999) & 120 & $\begin{array}{c}\text { Truncated Normal: } \\
\text { Stand. Dev. }=30, \\
\text { Min. }=50 \\
\text { Max. }=200\end{array}$ \\
\hline $\begin{array}{l}\text { Liquid Heat } \\
\text { Capacity }\end{array}$ & $\mathrm{J} / \mathrm{kg}-\mathrm{C}$ & (Sathyanarayana 1996) Refer to Table 3-2 & 3349 & - \\
\hline $\begin{array}{l}\text { Solid Heat } \\
\text { Capacity }\end{array}$ & $\mathrm{J} / \mathrm{kg}-\mathrm{C}$ & (Sathyanarayana 1996) Refer to Table 3-2 & 837 & - \\
\hline
\end{tabular}

headspace. For a given tank bump magnitude, the headspace volume obviously has a first-order effect on consequences by determining the peak overpressure. Headspace free volume is known by subtracting the waste volume, which is listed in Appendix A, from the total tank volume, which can be obtained from the Barton and Bingham (1999) database; the total tank volume as listed in the Barton and Bingham (1999) database includes the dome space and is not the designed waste capacity. This calculation gives the total free volume available in the headspace.

The size and nature of flowpaths from the tank headspace determine the final overpressure and magnitude of releases. These flowpaths are in the form of ventilation ducts, risers, cascade lines, pit cover, flanges, etc., as described in the rise configuration document (Alstad 1993).

Ventilation flowrate and duct diameter are listed in the Barton and Bingham (1999) database. Consequences depend on the flow resistance and decontamination factor provided by a riser HEPA filter. Releases are greatly mitigated if the HEPA filter remains intact. For the DST, the Barton and Bingham (1999) database lists 5 psig as the HEPA filter failure pressure and $99.96 \%$ as the efficiency.

Some tank-specific information for 241-AZ-101 and 214-AZ-102 are mentioned here because it will be used later. Units are presented in British or Sl as obtained from the original reference, for clarity in tracing. Pit and riser information is obtained from drawings (H-2-68304, H-2-68305, H-2-68423, and H-Unknown-1), and from Barton and Bingham (1999). 
Normal flowpaths between tank headspace and ambient are the filtered 8" diameter inlet and 20" diameter outlet. Unfiltered release paths from Barton and Bingham (1999) do not apply due to pit riser configuration details, as follows.

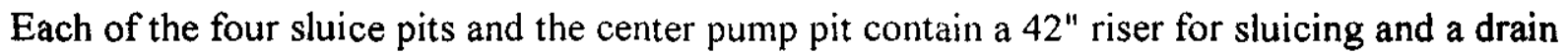
riser of 3" diameter in sluice pits and 4" diameter in the pump pit. The 42" risers terminate close to the tank dome and communicate with the headspace, while the drain risers extend to within five feet of the tank bottom and are immersed in waste. The 42 " risers have concrete and steel shield plugs that are 5 feet thick and weigh $8,500 \mathrm{lbs}$. or $3,864 \mathrm{~kg}$.

Steel flanges on these 42 " risers and shield plugs have 16 bolt holes of 7/8" diameter for sealing, but the sealing status of these risers is not known. If unsecured, a pressure difference of about $(3864 \mathrm{~kg})\left(9.81 \mathrm{~m} / \mathrm{s}^{2}\right) /\left(0.89 \mathrm{~m}^{2}\right)=42.5 \mathrm{kPa}$ from tank to ambient will cause the plugs to lift.

Sluice pits measure about $6.5^{\prime}$ wide $\times 8^{\prime}$ long $\times 7^{\prime}$ deep with pit covers of $2.0^{\prime}$ thickness in place. So open volume in sluice pits is about $10 \mathrm{~m}^{3}$ and the pressure to lift a pit cover is about $13.8 \mathrm{kPa}$ (using a concrete density of $2,300 \mathrm{~kg} / \mathrm{m}^{3}$ ). Pump pits have dimensions of about $8.5^{\prime}$ wide $\times 12^{\prime}$ long $\times 7^{\prime}$ deep with pit covers of $2.5^{\prime}$ in place, so open volume is about $20 \mathrm{~m}^{3}$ and cover lift pressure is about $17 \mathrm{kPa}$.

Following the Barton and Bingham (1999) assumption of $1 / 64^{\prime \prime}$ gap around unsealed risers, the leakage area for a single such riser is $\pi(42 \times 0.0254)(0.0254 / 64)=1.33 \times 10^{-3} \mathrm{~m}^{2}$, which is the equivalent of a $1.6^{\prime \prime}$ diameter hole. The reference pit leakage gap is $1 / 4^{\prime \prime}$, which applied to a sluice pit (perimeter $=29^{\prime}$ ) yields $87 \mathrm{in}^{2}=0.056 \mathrm{~m}^{2}$ and to a pump pit (perimeter $=41^{\prime}$ ) yields 123 in $^{2}=0.079 \mathrm{~m}^{2}$.

\subsection{FAILURE AND RECOVERY FREQUENCY DATA}

These types of data are required to judge the credibility of a tank bump event, considering the requisite physical conditions described above. In other words, these data are needed to assign the probability or annual frequency of attaining a non-convective layer with locally saturated conditions and a deep supernatant layer near the boiling point. This tank-specific frequency is determined in part by deterministic factors; e.g., supernatant depth and tank heat load are known to a reasonable certainty. Other conditions, such as equipment failures, external events, and human errors are inherently stochastic or uncertain as a practical matter. Conditions needed for a tank bump clearly require one of the following events:

- an external event (loss of power, seismic, lightning) that leads to loss of ventilation and heat up to saturation by self-heating, or

- $\quad$ equipment failure that lead to loss of ventilation and heat up to saturation by self-heating. 


\subsubsection{Recovery Time}

A first step to estimating the frequency of a tank bump is to calculate the time needed to heat the non-convective layer and supernatant to saturation, assuming that the initial conditions are steady-state temperatures with normal ventilation. The duration needed to allow waste selfheating to saturation is conservatively estimated by the equation for adiabatic heat-up:

$$
\frac{d T}{d t}=\frac{\dot{Q}}{\left(V_{N C} \rho_{N C} c_{N C}+V_{C L} \rho_{C L} c_{C L}\right)}
$$

where $\mathrm{V}$ is volume, $\rho$ is density, $\mathrm{c}$ is specific heat, $\mathrm{T}$ is the waste average temperature, and $\mathrm{Q}$ is the tank heat load rate due to self-heating. Subscripts NC and CL denote non-convective layer and supernatant (convective layer), respectively.

Adiabatic heatup calculations show that for most of the DST, the time needed to self-heat to saturation is very long relative to the time needed for recovery actions. This was demonstrated in Kummerer (1994), which lists the adiabatic time to boiling for DSTs as anywhere between one year and more than one thousand years. Kummerer (1994) considered all DSTs except 241-AY-101, 241-AY-102, 241-AZ-101, and 214-AZ-102. 241-SY-101 has the shortest time to boiling, with a value of 1.2 years. Many of the tanks with a large liquid volume and little solids, such as those in the AP farm, have time to boiling listed as greater than 1000 years.

Best-estimate calculations that credit heat conduction to underlying soil and radiative heat transfer and natural convection from the supernatant surface would in all likelihood demonstrate that most DSTs will never self-heat to boiling. Waters et al. (1991) considered such calculations for tank 241-AZ-101. In Waters' et al. (1991) HEATING7 calculations, two cases considered a 100-day outage of primary and annulus ventilation, as well as ALC operation, with initial conditions set to those prior to the mixing process. The first case considered a best-estimate effective supernatant conductivity $(150 \mathrm{Btu} / \mathrm{hr}-\mathrm{ft}-\mathrm{F})$ that reflected the mixing and small temperature gradients expected in the convective layer. The second case considered a pessimistic supernatant conductivity ( $16.7 \mathrm{Btu} / \mathrm{hr}-\mathrm{ft}-\mathrm{hr})$. A tank bump would not be induced in either case after an outage of 100 days, although temperatures are still rising at the end of the runs and eventual tank bump conditions cannot be ruled out.

In Section 6.0, best-estimate calculations for the time to bump conditions are made as part of screening criteria for a short list of tanks susceptible to bumps. The methods in Section 6.0 are suitable for hand calculation, but capture the essential aspects of the time to bump calculation. Equation (3-4) is modified by including upward and downward conduction losses, and the time to saturated conditions is estimated. To gauge the credibility of a tank bump, the time to bump conditions is compared to the time needed to recover the DST primary ventilation system and ancillary components. If the time to a tank bump greatly exceeds the time needed to recover, a tank bump is not credible. This evaluation is made on a tank-by-tank basis in Section 6.0. 


\subsubsection{Initiator Frequency}

In the case of self-heating then, loss of ventilation is required for an extended period. Loss of ventilation has four major initiating events: (1) random component failures or common cause failures, (2) loss of off-site power events (LOSP), (3) seismic events, and (4) lightning. The main information source for failure rates is the reliability, availability, and maintainability (RAM) of waste feed delivery from the 214-AZ-101 DST to the eventual customer (Carlson 1999). External accident initiators have been considered previously. Loss of off-site power was considered by Shultz (1994) in 1994, and most recently by Hunt (2000). A seismic hazard curve is given in Han (1996a and 1996b). Lightning strikes have been often considered as accident initiators (Cowley and Stepnewski 1994; Buck 1993; and Zach 1996). The crux has been some expression for the number of lightning strikes per unit area per unit time. These references are discussed further in Section 7.

\subsubsection{Corrective Maintenance and Restoration Times}

To judge the frequency or credibility of a tank bump scenario, the time to tank bump conditions given a loss of ventilation must be compared to the time needed to recover the ventilation system. In the safe storage scenarios, tank bumps are credible only in self-heating leads to bump conditions prior to ventilation system recovery. The information source used here is Carlson (1999), which provides a reliability, availability, and maintainability (RAM), study of waste feed delivery from the 241-AZ-101 tank to eventual customer. The assumption applied here is that repair times derived for the waste feed delivery RAM can be applied to the safe storage scenario.

The waste feed delivery RAM study identifies two types of recovery actions: restoration and corrective maintenance. A restoration activity is one in which a repair is not needed to recover the ventilation system. For example, using a redundant train while the primary one is under repair is a restoration function; manual operation in lieu of automatic actuation is another. A conclusion of the waste feed delivery RAM is that many ventilation system failures can be recovered by simple restoration activities which take no more than a week.

Corrective maintenance activities require repair time to recover the ventilation system. The waste feed delivery RAM identified probability distributions for the various corrective maintenance times. These times are a function of the system under repair (electrical, mechanical, HVAC, etc.), radiological exposure, spare parts availability, and the amount of planning or training required. These are considered in more detail in Section 7.0, which calculates the annual frequency or probability of a tank bump.

As a screening criterion, any tank with a time to boiling that is measured in years can be excluded from further concern. Carlson (1999) shows that mean repair times for the ventilation system are on the order of several days, with a month or so being the high ( $95^{\text {th }}$ percentile) value. For a worst case corrective maintenance time (one requiring pit access, remote handling, and extensive planning), the $95^{\text {th }}$ percentile for repair time is 336 days, but this activity is highly unlikely for DST ventilation system recovery. 
RPP-6213 REV 0

This page intentionally left blank. 


\subsection{TANK BUMP PHYSICAL MODELS AND CRITERIA}

Tank bumps are related to rapid steam generation in the waste liquid and the release of energy when the steam, in the form of a large bubble (or numerous bubbles), passes through the waste surface. These eruptions are not sufficient to threaten the mechanical integrity of the tank, but may lead to undesirable discharge of radioactive contaminants.

It is difficult to imagine a tank bump event in the absence of a deep layer of supernatant at a temperature approaching $100^{\circ} \mathrm{C}$ overlying a heat-producing normally non-convective waste layer. This concept will be developed and proven here in this section. Two physical scenarios for tank bump are deemed plausible and are investigated here:

In Section 4.1, we will demonstrate that tanks lacking a fairly deep, nearly saturated supernatant layer cannot have a significant tank bump. The scenario developed here is for gas injection from a (normally) non-convective lower waste layer into an overlying supernatant layer, Figure 4-1. The gas injection may be natural release of radiolytically and thermally generated noncondensible gases, such has hydrogen, or it may be gas injection due to some operation in the tank. A bump criterion for this scenario is the combination of significant gas injection, deep supernatant layer, and nearly saturated supernatant layer.

In Section 4.2, we will demonstrate that a buoyant displacement event (Figure 4-2) is a mechanism that can result in a significant steam bump providing that the non-convective layer is capable of self-heating to nearly its boiling point at the local static pressure and providing that noncondensible gas generation can bring the non-convective layer to a buoyant state. It will be shown that the latter requirement eliminates the high self-heat rate tanks of concern from exhibiting steam bump behavior.

Two additional physical scenarios for tank bump postulated over the years are deemed not plausible based on analysis presented in Appendix B:

In Appendix B.1 we investigate a hypothetical scenario for steam bump due to interstitial liquid super heating in stationary sludge, Figure B-1. The purpose of this investigation is to prove that significant sudden release of vapor cannot occur from a stationary sludge layer alone.

In Appendix B. 2 we examine steam bubble growth due to local convection in an otherwise non-convective layer, Figure B-3. This scenario corresponds to concerns raised at one time for tank 241-C-106, when an increase in waste temperature was observed following a process test. Local convection, or fumaroles as the phenomenon was called at the time, are shown not to be a source for a significant steam bump. 
RPP-6213 REV 0

Figure 4-1. Steam Bump By Gas Injection.

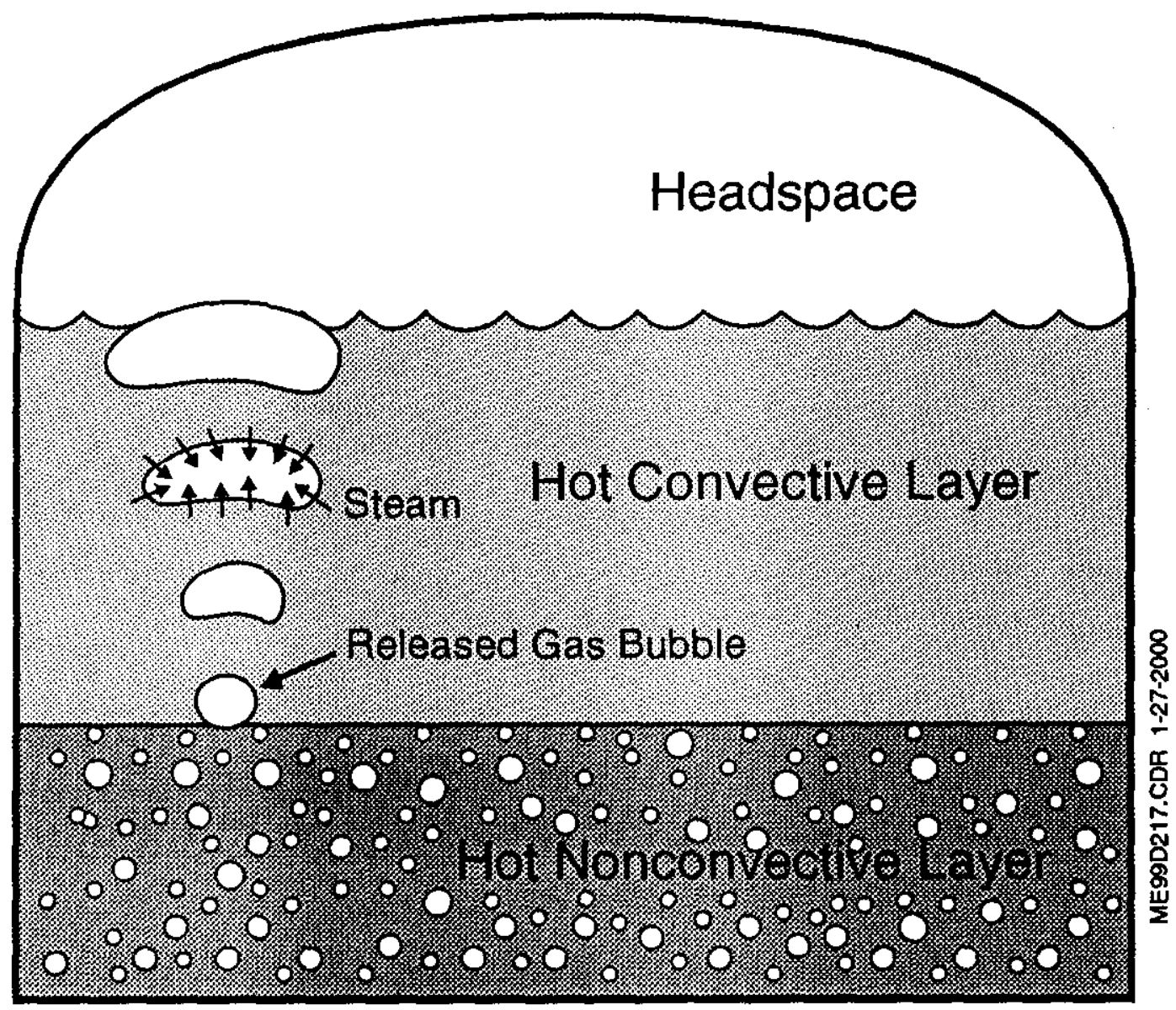


Figure 4-2. Steam Bump During a Buoyant Displacement.
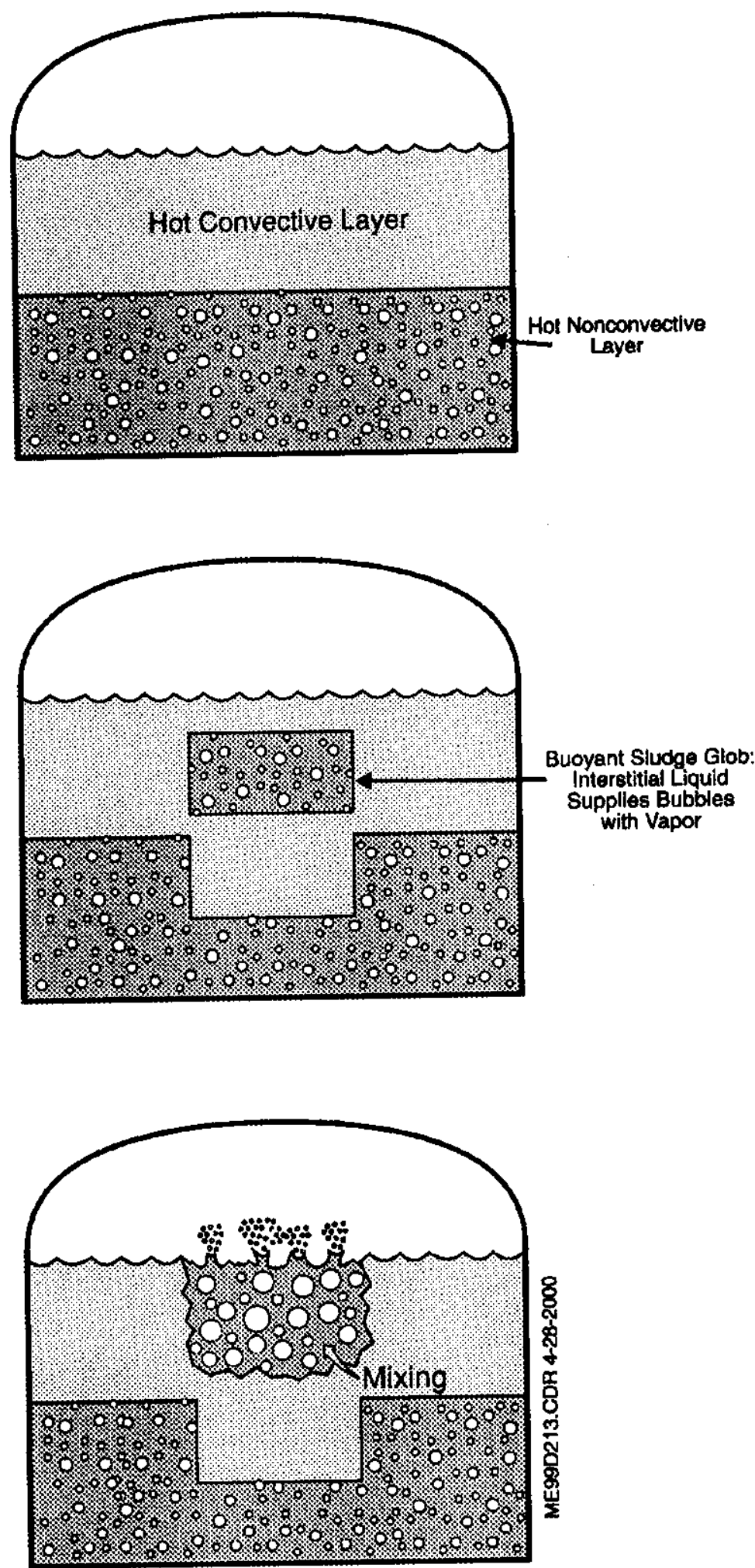


\subsection{STEAM BUMPS BY GAS INJECTION}

Consider the growth of a gas cavity of volume $V_{b}(0)$ that suddenly appears at the bottom of a supernatant layer of depth $\mathrm{H}$ and uniform temperature $\mathrm{T}_{\ell}$, Figure 4-1. The cavity is filled with the vapor of the surrounding water and by the noncondensible gas generated in the underlying non-convective layer. It is well known that natural convection currents within the water layer are more than sufficient to maintain the liquid at a nearly isothermal condition. The magnitude of the cavity expansion during its ascent through the liquid layer is a measure of the severity of the tank bump event and this is the problem treated below.

In keeping with our objectives, we consider the transient growth of a spherical cavity (bubble), initially containing the gas and water vapor (steam) it gradually accumulated before it was released at the bottom of the supernatant layer. The initial partial pressure of steam in the bubble, $P_{e q}\left(T_{\ell}\right)$, is specified by demanding thermodynamic equilibrium between steam and liquid at the bubble surface. As the bubble rises into regions of lower hydrostatic pressure, due to the presence of noncondensible gas (hereafter referred to as "gas"), the bubble expands and the steam partial pressure in the interior of the bubble falls below the equilibrium steam partial pressure at the bubble surface. This results in a concentration gradient within the bubble and steam transport from the surface of the bubble to its interior. In turn, water must evaporate and pass from the supernatant and into the bubble. The evaporation process tends to cool the liquid so that its temperature decreases from $T_{\ell}$ in the immediate vicinity of the bubble surface and a thermal boundary layer develops on the liquid side of the bubble. Thus the growth of the bubble is controlled by heat and mass transport; it is limited by the rate at which latent heat can be supplied by liquid convection at the bubble surface and by the rate at which steam can diffuse (convect) into the bubble from its surface. The bubble growth problem is obviously complicated.

To simplify matters and still retain a conservative approach, it is assumed that bubble growth is limited by steam transport within the bubble. The resistance to growth imposed by the thermal boundary layer on the liquid side is ignored. From this assumption it follows that the steam partial pressure at the bubble surface equals $\mathrm{P}_{\mathrm{eq}}\left(\mathrm{T}_{\ell}\right)$ throughout its motion. Thus, the rate at which steam enters the bubble is given by a mass transport law of the form

$$
\frac{\mathrm{dm}_{\mathrm{v}}}{\mathrm{dt}}=4 \pi \mathrm{r}^{2} \mathrm{~h}_{\mathrm{m}} \rho_{\text {mix }} \ln \left[1+\frac{\rho_{\mathrm{eq}}\left(\mathrm{T}_{\ell}\right)-\rho_{\mathrm{v}}}{\rho_{\text {mix }}-\rho_{\mathrm{eq}}\left(\mathrm{T}_{\ell}\right)}\right]
$$

where $m_{v}$ is the instantaneous mass of steam within the bubble at time $t, r$ is the bubble radius, $\rho_{v}$ is the bulk steam density within the bubble, $\rho_{\text {mix }}$ is the bulk gas/vapor mixture density within the bubble, $\rho_{\mathrm{eq}}\left(\mathrm{T}_{\ell}\right)$ is the equilibrium density of steam at the inside surface of the bubble evaluated at the supernatant temperature $T_{\ell}$, and $h_{m}$ is the coefficient for steam transport from the bubble surface into its interior.

A maximum rate of bubble-gas side mass transfer can be predicted by postulating a model in which the bubble interior is well mixed and the entire resistance to mass transfer restricted to a thin gas/steam boundary layer at the bubble surface. Such a model was analyzed by 
Ruckenstein et al. (1971). A Hill's vortex bubble flow field was linearized near the bubble surface and the final expression for $h_{m}$ was found to be

$$
h_{m}=\left(\frac{2 U_{b} D}{\pi r}\right)^{1 / 2}
$$

where $U_{b}$ is the bubble rise velocity relative to the surrounding liquid and $D$ is the binary diffusion coefficient for the bubble mixture (steam + gas).

The mass of vapor in the bubble in equation (4-1) can be expressed as the product of the bulk (bubble-interior) steam density and bubble volume $V_{b}$

$$
\mathrm{m}_{\mathrm{v}}=\rho_{\mathrm{v}} \mathrm{V}_{\mathrm{b}}
$$

Assuming that steam behaves as an ideal gas, we may write

$$
\begin{aligned}
& \rho_{\mathrm{eq}}\left(\mathrm{T}_{\ell}\right)=\frac{\mathrm{P}_{\mathrm{eq}}\left(\mathrm{T}_{\ell}\right)}{\mathrm{RT} \mathrm{T}_{\ell}} ; \rho_{\text {mix }}=\frac{\mathrm{P}_{\text {mix }}}{\mathrm{RT}_{\ell}} \\
& \rho_{\mathrm{v}}=\frac{\mathrm{P}_{\mathrm{v}}}{\mathrm{RT} \mathrm{T}_{\ell}}
\end{aligned}
$$

where $\mathrm{R}$ is the ideal gas constant for steam, $\mathrm{P}_{\text {mix }}$ is the total bulk pressure within the bubble, and $P_{v}$ is the steam partial pressure in the well-mixed bubble interior. The second relation in equation (4-4) is based on the justifiable assumption that the steam contribution to the value of the bubble mixture molecular weight far exceeds that due to the inert gas. The bubble is assumed to rise through the supernatant as part of an ensemble (cluster) of bubbles. A constant "terminal" speed of the cluster is assumed and denoted by the symbol U. Thus time may be replaced by vertical distance traversed by the bubble as follows:

$$
t=\frac{z}{U}
$$

where $\mathrm{z}$ is measured from the bottom of the supernatant pool. The constant $U$ assumption will be relaxed later on when a more complete steam bump model is constructed. Combining equations (4-1) to (4-6) gives

$$
\frac{d\left(P_{v} V_{b}\right)}{d z}=\left(\frac{24 D U_{b}}{U^{2}} \cdot V_{b}\right)^{1 / 2} P_{\text {mix }} \ln \left[1+\frac{P_{e q}\left(T_{\ell}\right)-P_{v}}{P_{\text {mix }}-P_{e q}\left(T_{\ell}\right)}\right]
$$

To close the problem, we need a relationship between $P_{v}$ and the bubble volume $V_{b}$. This relationship is obtained by the following liquid (supernatant) statics analysis. The gas partial 
pressure $P_{g}$ plus the steam pressure $P_{v}$ within the bubble must equal the local hydrostatic pressure imposed by the supernatant; that is,

$$
P_{\text {mix }}=P_{v}+P_{g}=P_{h s}+\rho_{\ell} g(H-z)
$$

where $P_{h s}$ is the pressure in the tank headspace, $g$ is the gravitational constant, and $\rho_{\ell}$ and $\mathrm{H}$ are, respectively, the density and depth of the supernatant layer. The mass of inert gas in the bubble remains constant so that by virtue of the ideal gas law

$$
P_{g}(0) V_{b}(0)=P_{g} V_{b}
$$

where $P_{g}(0)$ and $V_{b}(0)$ denote the values of $P_{g}$ and $V_{b}$ at the bottom of the supernatant layer (i.e., at $\mathrm{z}=0$ ). Evaluating equation (4-8) at $\mathrm{z}=0$ and making the reasonable assumption that the bubble emerges from the non-convective layer with an equilibrium concentration of steam throughout its volume, so that,

$$
P_{v}(0)=P_{e q}\left(T_{\ell}\right)
$$

yields

$$
\mathrm{P}_{\mathrm{eq}}\left(\mathrm{T}_{\ell}\right)+\mathrm{P}_{\mathrm{g}}(0)=\mathrm{P}_{\mathrm{hs}}+\rho_{\ell} \mathrm{gH}
$$

Eliminating $P_{g}$ between equations (4-8) and (4-9) and inserting $P_{g}(0)$ from equation (4-11) into the result gives

$$
\frac{\mathrm{V}_{\mathrm{b}}}{\mathrm{V}_{\mathrm{b}}(0)}=\frac{\mathrm{P}_{\mathrm{hs}}+\rho_{\ell} \mathrm{gH}-\mathrm{P}_{\mathrm{eq}}\left(\mathrm{T}_{\ell}\right)}{\mathrm{P}_{\mathrm{hs}}+\rho_{\ell} \mathrm{g}(\mathrm{H}-\mathrm{z})-\mathrm{P}_{\mathrm{v}}}
$$

Equations (4-7) and (4-12) are sufficient to solve for the unknowns $V_{b}$ and $P_{v}$ as a function of elevation $z$. Unfortunately, the system cannot be integrated in closed form; a numerical solution of equation (4-7) is required. Note, however, that in the limit of no mass transfer resistance to bubble growth, equation (4-12) alone can be used to calculate the bubble volume as a function of vertical distance. This is accomplished by replacing $P_{v}$ on the right-hand side of equation (4-12) with $P_{e q}\left(T_{l}\right)$. Note also that $P_{h s}$ in equation (4-12) is not constant during a steam bump but increases in response to the rising and expanding bubble cluster within the supernatant. For the purpose of illustrating the conditions required for a strong steam bump due to gas injection, it is convenient to first ignore the coupling between the bubble cluster and the headspace atmosphere and assume that $P_{h s}$ remains at its near-atmospheric value.

The various property values used in the illustrative calculations are: $P_{\mathrm{hs}}=0.1013 \mathrm{MPa}, \rho_{\ell}=$ $1100 \mathrm{~kg} \mathrm{~m}^{-3}, \mathrm{D}=9.2 \times 10^{-5} \mathrm{~m}^{2} \mathrm{~s}^{-1}$, and $\mathrm{U}=1.0 \mathrm{~m} \mathrm{~s}^{-1}$. The diffusion coefficient $\mathrm{D}$ for mass transfer within the bubble was estimated for a hydrogen gas/steam mixture at $373 \mathrm{~K}$ and $0.14 \mathrm{MPa}$ pressure by the method outlined in Reid and Sherwood (1966) for polar/non-polar gas pairs. The maximum possible bubble velocity relative to the cluster liquid is the terminal rise velocity of an isolated bubble in a quiescent liquid. The terminal rise velocity of an isolated 
bubble is typically about $U_{b}=0.2 \mathrm{~ms}^{-1}$. The rise velocity of a collection of bubbles can be estimated from the formula

$$
\mathrm{U}=0.68\left(\mathrm{gD}_{\text {eff }}\right)^{1 / 2}
$$

where $\mathrm{D}_{\text {eff }}$ is the diameter of a large, fictitious bubble having the same volume as the total volume of the bubble ensemble (Moody 1986). The formula implies a bubble ensemble rise velocity of at least $U=1.0 \mathrm{~m} \mathrm{~s}^{-1}$ for gas injections capable of producing a steam bump. Finally, the equilibrium vapor pressure of steam $\mathrm{P}_{\text {eq }}\left(\mathrm{T}_{\ell}\right)$ was estimated as a function of supernatant temperature $T_{\ell}$ by using steam table values.

Figures 4-3 and 4-4 illustrate the results of the numerical calculations. In both figures, the bubble volume $\mathrm{V}_{\mathrm{b}}(\mathrm{H})$ at the end of the bubble's ascent through the supernatant layer divided by its initial volume $\mathrm{V}_{b}(0)$ at the bottom of the layer is plotted against the depth $\mathrm{H}$ of the layer. Figure 4-3 shows the effect of supernatant temperature on bubble expansion while Figure 4-4 shows the effect of initial bubble size (diameter $\mathrm{d}_{0}$ ) on bubble expansion. Mass transfer resistance becomes important as the supernatant temperature $T_{l}$ approaches its boiling point $\left(100^{\circ} \mathrm{C}\right)$. Clearly, the bubble expansion ratio is sensitive to the initial bubble size. Observations of retained gas in simulants and actual wastes indicate that the average bubble diameter is approximately $1.0 \mathrm{~mm}$ and that the upper end of the size range of retained round gas bubbles in waste is about $5.0 \mathrm{~mm}$ (Gauglitz et al. 1996). The larger bubbles should serve as the sites from which the bubbles grow during a spontaneous gas release event or waste disturbing operation as the free energy required to initiate bubble growth decreases by an amount proportional to the volume of the embryo bubble. It follows that conservative estimates of the bubble expansion ratio may be based on the choice $\mathrm{d}_{0}=1.0 \mathrm{~mm}$. It should be mentioned that during a spontaneous gas release event there will be a significant reduction in the bubble size (and in total release void volume) due to vapor condensation as the bubbles are transported from the non-convective layer to the relatively cooler supernatant.

Regardless of the dimensions of the bubbles, it is clear from Figures 4-3 and 4-4 that a significant tank bump event by gas injection requires a deep supernatant layer whose temperature approaches the boiling point.

In the preceding example, the ambient temperature was assumed to be a standard atmosphere. Also, the effect of salt in solution to increase the boiling point was neglected. These simplifications do not change conclusions when values pertinent to a given tank and ambient pressure are used.

While the volume expansion ratio $\mathrm{V}_{\mathrm{b}}(\mathrm{H}) / \mathrm{V}_{\mathrm{b}}(0)$ results given in Figures 4-3 and 4-4 were obtained for a single bubble in a bubble ensemble, the results are also applicable for the bubble ensemble itself providing that bubble coalescence is ignored and an effective mean bubble size is specified. For the bubble ensemble case, $V_{b}(0)$ and $V_{b}(H)$ in the volume expansion ratio refer respectively to the total volume of the bubble ensemble at the bottom of the supernatant and the total volume of the bubble ensemble upon its arrival at the waste surface. Therefore, to calculate the absolute bubble volume produced as a result of a gas injection and bubble ascent, we must 
Figure 4-3. Bubble Expansion Ratio Versus Depth of Supernatant Pool; Pool Temperature $T_{\lambda}$ as a Parameter. Dashed curves refer to zero mass transfer resistance.

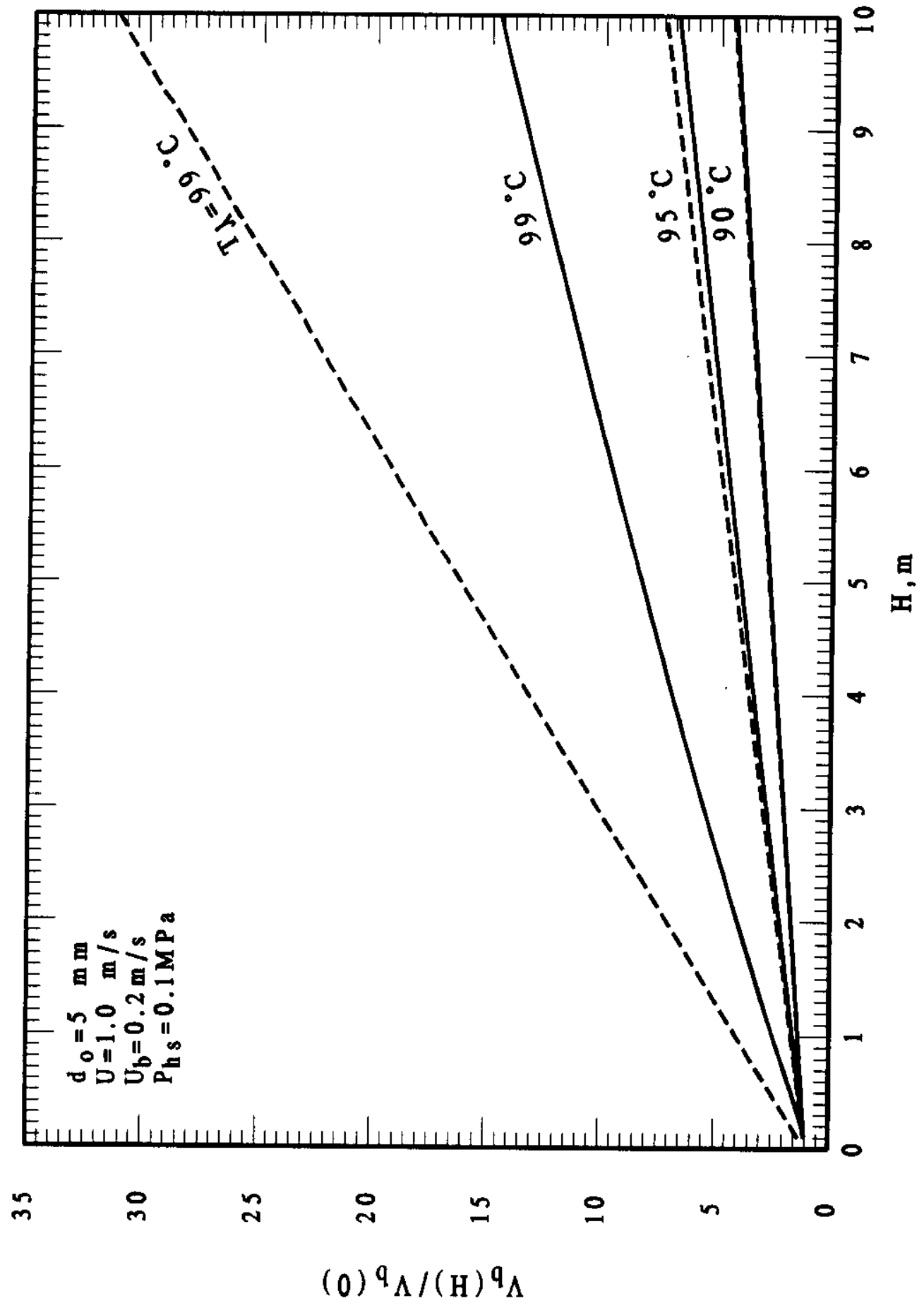


Figure 4-4. Bubble Expansion Ratio Versus Depth of Supernatant Pool; Initial Bubble Diameter as a Parameter. Dashed curve refers to zero mass transfer resistance.

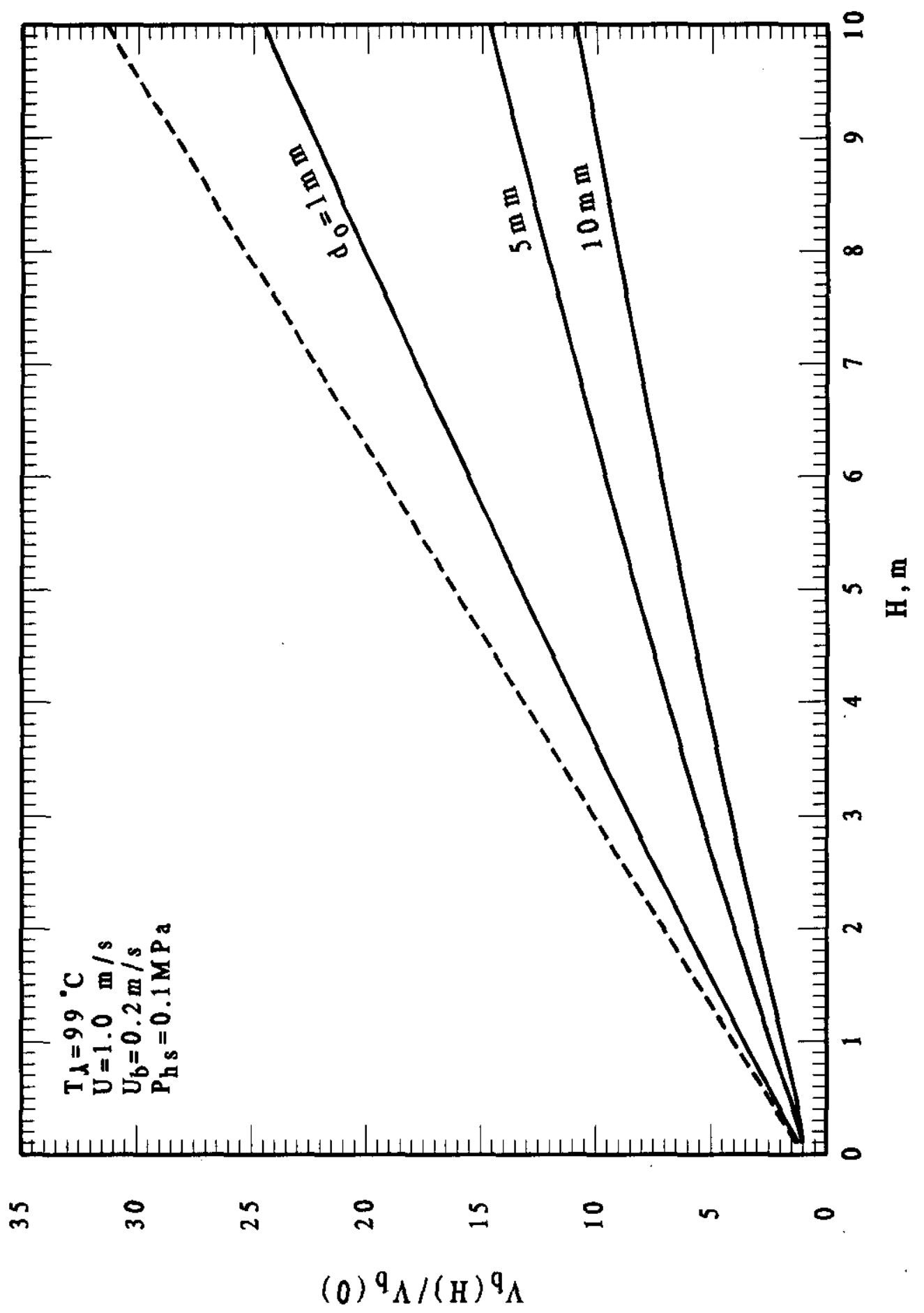


estimate $V_{b}(0)$. The application of the bubble growth theory presented in the foregoing to the behavior of a cluster of bubbles is postponed until Section 5.0 where a tank bump consequence model is described and exercised.

An underlying assumption in the calculation is that the supernatant temperature is uniform; this is typically taken as obvious as mentioned above and in the discussion of Section 3.1. The point is proven here to illustrate how rigid the requirement is for the entire supernatant layer to be nearly saturated. For turbulent natural convection in a liquid layer with a free upper surface, the relationship between heat flux and core to surface temperature difference is (Katsaros et al. 1977):

$$
\mathrm{Nu}=0.156 \mathrm{Ra}^{0.33}
$$

where $\mathrm{Nu}=\mathrm{hL} / \mathrm{k}$, and

$$
\mathrm{Ra}=\mathrm{g} \beta \Delta \mathrm{TL}^{3} / \alpha \nu
$$

Heat flux is given by the product $h \Delta T$, and is equal to the amount of tank power $\mathrm{Q}_{\mathrm{u}}$ lost upward divided by area $\mathrm{A}$,

$$
\frac{Q_{u}}{A}=k\left(\frac{g \beta}{\alpha v}\right)^{\frac{1}{3}}(\Delta T)^{4 / 3}
$$

For a representative (and high) power of $10 \mathrm{~kW}$, and typical values as follows, $A=411 \mathrm{~m}^{2}$, $\mathrm{k}=0.68 \mathrm{~W} / \mathrm{m} / \mathrm{K}, \mathrm{g}=9.81 \mathrm{~m} / \mathrm{s}^{2}, \beta=6 \times 10^{-4} \mathrm{~K}^{-1}, \alpha=3 \times 10^{-5} \mathrm{~m}^{2} / \mathrm{s}, v=3 \times 10^{-5} \mathrm{~m}^{2} / \mathrm{s}$, the temperature difference is only $\Delta \mathrm{T}=0.29 \mathrm{~K}$. The total temperature difference between the bottom of the layer and the top of the layer would double this value, or about $0.6 \mathrm{~K}$. For higher upward heat flux, typically required for the supernatant layer to be nearly saturated, the temperature difference would be even lower.

\subsection{STEAM BUMP BY BUOYANT DISPLACEMENT}

A buoyant displacement occurs when a gas (vapor)-generating and gas (vapor)-trapping nonconvective layer is covered by a relatively thick layer of initially less dense supernatant.

Eventually, gas (vapor) generation in the lower layer leads to a density inversion and the buoyant displacement event. The temperature $T_{n c}$ in the non-convective layer usually exceeds the temperature $T_{l}$ in the overlying supernatant. Using the physical properties of water it is possible for the non-convective layer to reach a temperature of $120^{\circ} \mathrm{C}$ at a depth $10 \mathrm{~m}$ below the free surface of the waste. Owing to natural convection the supernatant temperature cannot exceed its boiling point of $100^{\circ} \mathrm{C}$ at atmospheric pressure. During a buoyant displacement in a "hot tank", the bubbles trapped in the rising, hot non-convective layer material grow by converting the hot interstitial liquid $\left(>100^{\circ} \mathrm{C}\right)$ into steam while a bubble injected into the supernatant grows by accumulating vapor from the relatively low temperature supernatant $\left(<100^{\circ} \mathrm{C}\right)$. The model derived in the previous sub-section can be used to illustrate the effect of the temperature of the 
host liquid (supernatant or non-convective buoyant material) on the bubble volume expansion ratio $V_{b}(H) / V_{b}(0)$. The results are shown in Figure 4-5 where we see that the severity of a tank bump event increases dramatically once the non-convective layer temperature exceeds the maximum supernatant temperature $T_{n c}=100^{\circ} \mathrm{C}$.

The results in Figure 4-5 pertain only to representative cluster-bubble behavior beneath a constant pressure headspace. It will be seen later on that owing to the pressurization of the tank headspace and mixing between the buoyant parcel of initially non-convective material and the surrounding supernatant, the bubble volume expansion ratios achieved within the buoyant parcel during a tank bump are much smaller than those presented in Figure 4-5.

As already pointed out in Section 3, most of the Hanford site tanks have little potential for a tank bump because either their heat loads are too low to heat the waste to its boiling point or the time required to self-heat to the boiling point is very long compared with the time required to repair the equipment failure. Possible exceptions are tanks 241-AZ-101, 241-AZ-102, and 241-AY-102, which have rather large non-convective layer heat generation rates and for which boiling may not be incredible; this is discussed in Section 6 . The question remains as to whether these tanks will exhibit a buoyant displacement after their non-convective layer is heated to the boiling temperature.

Meyer and Wells (2000) derived models that give criteria that must be satisfied in order for buoyant displacements to occur. Briefly, they developed an equation for the vertical void fraction profile within the non-convective layer based on a balance between internal gas generation and the rate at which gas is released at the top of the layer. The integrated average of this void fraction profile is compared with the neutral buoyant void fraction to determine whether a buoyant displacement may occur at some point during the transient (quasi-steady) void growth period. Two limiting case solutions were found for the void fraction profile and the corresponding criteria for a buoyant displacement are

$$
\frac{\mathrm{C}_{1} \mathrm{~h}_{\mathrm{NCL}}^{2}}{\rho_{\mathrm{NCL}}-\rho_{\mathrm{CL}}}\left(\frac{\mathrm{G} \mathrm{T}}{\mathrm{P}_{\mathrm{NCL}}}\right)^{1 / 3}>1
$$

for a uniform bubble nucleation rate and a zero initial void fraction, and

$$
\frac{\mathrm{C}_{2}}{\rho_{\mathrm{NCL}}-\rho_{\mathrm{CL}}}\left(\frac{\rho_{\mathrm{NCL}}^{2} \mathrm{~h}_{\mathrm{NCL}}^{2} \mathrm{GT}}{\mathrm{P}_{\mathrm{NCL}}}\right)^{1 / 3}>1
$$

for an assumed bubble flux at the lower boundary and zero internal nucleation rate. In equations (4-16) and (4-17), $\rho_{\mathrm{NCL}}$ and $\rho_{\mathrm{CL}}$ are the density of the non-convective layer and the density of the convective layer $\left(\mathrm{kg} \mathrm{m}^{-3}\right)$, respectively, $\mathrm{G}$ is the molar gas generation rate per unit volume of non-convective layer $\left(\mathrm{g}\right.$-mole $\left.\mathrm{m}^{-3} \mathrm{day}^{-1}\right), \mathrm{T}$ is the average temperature of the non-convective layer $(\mathrm{K}), \mathrm{P}_{\mathrm{NCL}}$ is the average pressure of the non-convective layer (atm), and $\mathrm{h}_{\mathrm{NCL}}$ is the depth of the non-convective layer $(\mathrm{m})$. The constants $\mathrm{C}_{1}$ and $\mathrm{C}_{2}$, each with dimensional units, are empirically adjusted so that all the double-shell tanks with observed buoyant displacements obey 
Figure 4-5. Bubble Expansion Ratio Versus Convective Layer Temperature $\left(<100^{\circ} \mathrm{C}\right)$ or Buoyant Parcel Temperature $T_{n c}$ During Buoyant Displacement.

Dashed curve refers to zero mass transfer resistance.

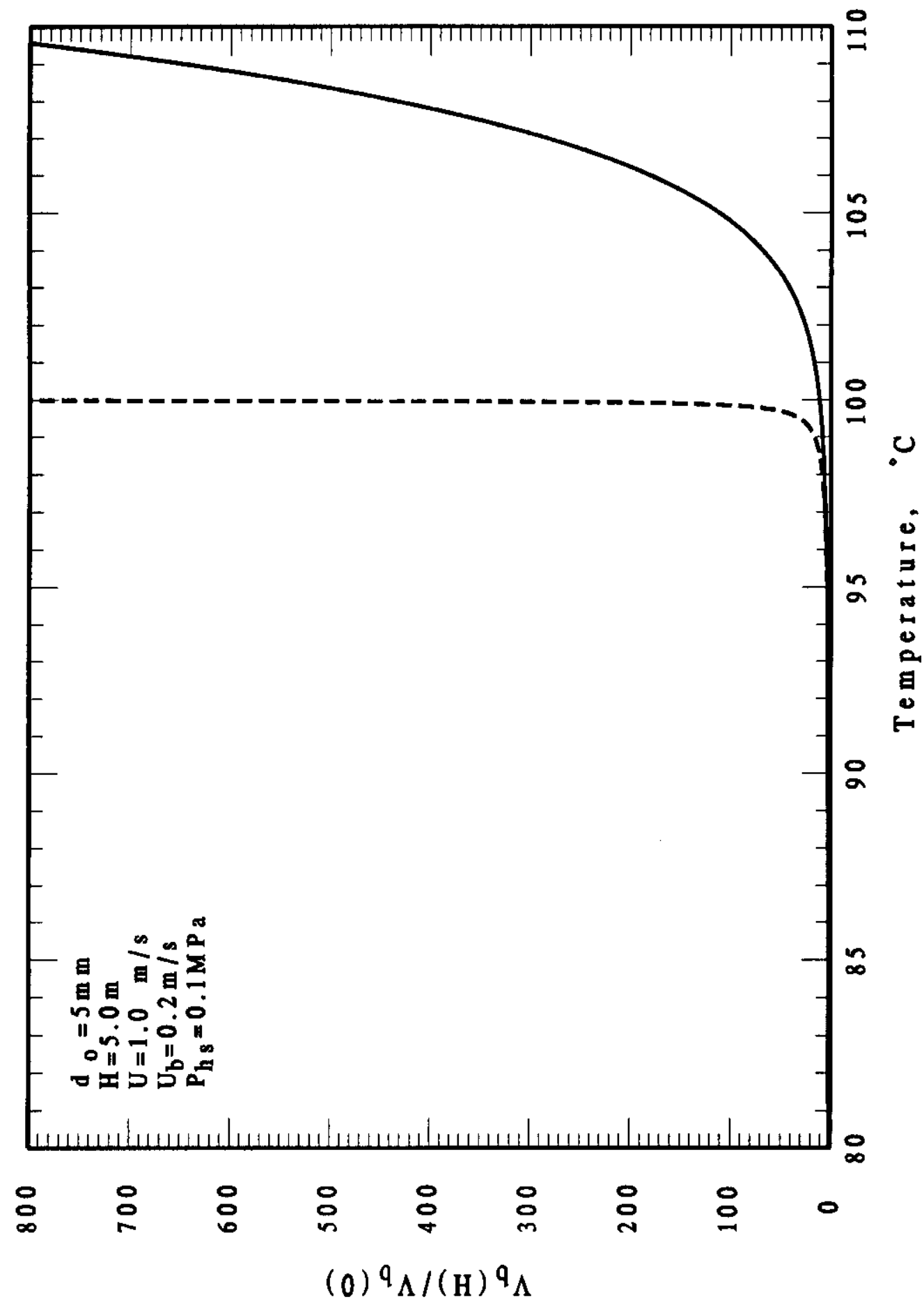


the criteria given by equations (4-16) and (4-17). Equation (4-17) provides much more conservative results than equation (4-16). However, equation (4-16) yields a better representation of double-shell tank buoyant displacement behavior (Stewart 2000) and is chosen here for application to tanks 241-AZ-101, 241-AZ-102, and 241-AY-102. The value of the constant in equation (4-16) based on the most recent tank data is $C_{1}=18.5$ (Stewart 2000).

The gas generation rate originally used in equation (4-16) is noncondensable gas generation by radiolysis and thermal decomposition. It is of interest to evaluate equation (4-16) when $G$ is based on steam generation only, and then, when $\mathrm{G}$ is based on noncondensable gas generation only. In both evaluations, the non-convective layer is presumed to be at its boiling temperature at the local static pressure. The steam production rate $\mathrm{G}_{\mathrm{v}}$ may be approximated by

$$
\mathrm{G}_{\mathrm{v}}=\frac{\dot{\mathrm{Q}}_{\mathrm{NCL}}^{m}}{\mathrm{~h}_{\mathrm{fv}} \mathrm{M}_{\mathrm{v}}}
$$

where $\dot{\mathrm{Q}}_{\mathrm{NCL}}^{m}$ is the heat generation rate per unit volume of non-convective layer, $\mathrm{h}_{\mathrm{fv}}$ is the latent heat of evaporation of water, and $\mathrm{M}_{\mathrm{v}}$ is the molecular weight of water. The noncondensable gas generation rate at the boiling point $\mathrm{T}_{\mathrm{bp}}$ (evaluated at the mid-plane of the non-convective layer) is obtained by using the Arrhenius equation to extrapolate the known gas generation rate at the prevailing non-convective layer temperature $T_{0}$ to its value at $T_{b p}$ :

$$
G_{g}\left(T_{b p}\right)=G_{g}\left(T_{0}\right) \exp \left(-\frac{T_{a c t}}{T_{b p}}+\frac{T_{a c t}}{T_{0}}\right)
$$

where the activation temperature $\mathrm{T}_{\mathrm{act}}(=\mathrm{E} / \mathrm{R})$ is $1.074 \times 10^{4}(\mathrm{Hu} 1999)$.

Table 4-1 lists the predicted values of $G_{v}$ and $G_{g}$ for tanks 241-AZ-101, 241-AZ-102, and 241-AY-102. It is obvious from the table that the steam production rate is much larger than the noncondensable gas generation rate. While the boiling temperature may be increased by a few degrees due to dissolved salt, this has only a minor effect.

\begin{tabular}{|c|c|c|c|c|c|c|}
\hline $\begin{array}{c}\text { Tank, } \\
241-\end{array}$ & $\begin{array}{l}\text { Prevailing } \\
\text { Temp. } T_{0} \\
\text { (K) }\end{array}$ & $\begin{array}{c}\text { Boiling } \\
\text { Temp. } \\
T_{\text {bp }}(K)\end{array}$ & $\begin{array}{l}\dot{\mathbf{Q}}_{\mathrm{WCL}}^{m} \\
\left(\mathbf{W} / \mathbf{m}^{3}\right)\end{array}$ & $\begin{array}{c}G_{g}\left(T_{0}\right) \\
\left(\frac{g-m o l e}{m^{3} \text { day }}\right)\end{array}$ & $\begin{array}{c}G_{g}\left(T_{b p}\right) \\
\left(\frac{g-\text { mole }}{m^{3} \text { day }}\right)\end{array}$ & $\begin{array}{c}G_{v} \\
\left(\frac{g-\text { mole }}{m^{3} \text { day }}\right)\end{array}$ \\
\hline$A Z-101$ & 345 & 392 & 260 & $6.12 \times 10^{-3}$ & 0.26 & 567 \\
\hline AZ-102 & 355 & 390 & 99 & $1.26 \times 10^{-2}$ & 0.19 & 216 \\
\hline$A Y-102$ & 350 & 384 & 31 & $1.80 \times 10^{-2}$ & 0.27 & 68 \\
\hline
\end{tabular}

Table 4-1. Gas and Steam Generation Rates in the Non-Convective Layer. 
The results of the buoyant displacement criterion calculations from equation (4-16) are listed in Table 4-2.

Table 4-2. Buoyant Displacement Model (equation (4-16)) Predictions for the High-Power Double-Shell Tanks.

\begin{tabular}{|c|c|c|c|c|c|c|}
\hline Tank & $\begin{array}{c}\text { PNCL } \\
\left(\mathrm{kg} \mathrm{m}^{-3}\right)\end{array}$ & $\begin{array}{c}\rho_{C L} \\
\left(\mathrm{~kg} \mathrm{~m}^{-3}\right)\end{array}$ & $\begin{array}{c}h_{N C L} \\
(\mathrm{~m})\end{array}$ & $\begin{array}{l}\text { P NCL }_{\text {(atm) }} \\
\text { (atm }\end{array}$ & $\begin{array}{c}\text { LHS}^{\star} \\
\text { equation } \\
(4-16): \\
G=G_{V}\end{array}$ & $\begin{array}{c}\text { LHS* }^{*} \\
\text { equation } \\
(4-16) \text { : } \\
G=G_{g}\end{array}$ \\
\hline 241-AZ-101 & 1690 & 1190 & 0.43 & 1.91 & 0.33 & 0.026 \\
\hline $241-A Z-102$ & 1490 & 1100 & 0.96 & 1.79 & 1.60 & 0.15 \\
\hline 241-AY-102 & 1480 & 1080 & 1.72 & 1.50 & 3.55 & 0.56 \\
\hline
\end{tabular}

An examination of the last column of Table 4-2 indicates that even in the presence of steam generation 241-AZ-101 fails to satisfy the buoyant displacement criterion, owing to its shallow non-convective layer. It is recognized that the Meyer and Wells (2000) criterion is valid at nonconvective layer boiling conditions only if the gas retention and gas migration properties of the non-convective layer remain the same during boiling of the layer. The criterion is probably valid during the period when the layer is heated from its initial, steady-state temperature to its boiling temperature and noncondensable gas generation is the dominant mode of void production. The last two columns of Table 4-2 show that buoyant displacements in the subject tanks can only occur by steam generation. Noncondensable gas generation alone cannot bring the nonconvective layer to a buoyant condition. However, steam condensation brought on by mixing of the supernatant with the buoyant materials (see below) will prevent the buoyant displacement from developing into a steam bump.

A postulated buoyant displacement in tanks 241-AZ-101, 241-AZ-102, or 241-AY-102 implies a weak (low-yield-strength) non-convective layer. In these tanks, the density difference between the non-convective layer and the overlying supernatant is large owing to the absence of significant quantities of dissolved salt. Consequently, the neutral buoyancy void fraction is high $(\sim 0.3)$. The experimental evidence (Gauglitz et al. 1996) suggests that if the waste has a high yield strength, the gas bubbles will connect and form a continuous path at void fractions below the neutral buoyancy void fraction. If the waste in tanks 241-AZ-101, 241-AZ-102, or 241-AY-102 is stiff, these tanks do not pose a buoyant-displacement-steam bump concern. Thus, a prerequisite for a steam bump is a low non-convective-layer-yield strength of the order of the known yield strengths of the non-convective layers in the six DSTs that exhibit buoyant displacement, say $\tau_{y} ; 100 \mathrm{~Pa}$. The available buoyant energy is more than sufficient to overcome the $100 \mathrm{~Pa}$ yield strength and rapidly transform the non-convective material in the rising parcel into a Newtonian fluid (Meyer et al. 1997). This transformation is immediately followed by the mixing of the buoyant parcel material with the surrounding sub-cooled supernatant. 
Intense mixing of the structurally weak, steam-void containing buoyant parcel with the surrounding supernatant will begin just as the parcel rises from the non-convective layer (see Appendix C). Mixing is caused by a vertical buoyancy-dominated, turbulent diffusion mechanism (Epstein and Burelbach 2000a). The density difference between the buoyant parcel and the surrounding supernatant, combined with the very low initial momentum of the buoyant release, causes the inward flow of supernatant so that an unstable density gradient persists above the release area. Consequently, a density-gradient driven vertical mixing zone is quickly established between the rising parcel of previously non-convective material and the overlying supernatant. It is pertinent to note here that the top 10 to $50 \mathrm{~cm}$ of the parcel is sub-cooled and has a low void fraction. This parcel "cover" is non-buoyant and stiff with respect to the convective layer. However, it is reasonable to believe that the cover will move some distance to the side to allow the underlying, buoyant, and flowing portion of the parcel to rise into the supernatant. Thus, vertical mixing between the steam-bearing material and supernatant will still occur, although over an area that is smaller than the horizontal extent of the parcel.

The temperature at the top of the mixing layer $T_{\text {mix }}$ as a function of the supernatant temperature $T_{C L}$ and the temperature $T_{b p}$ of the non-convective layer may be determined from Epstein and Burelbachs' (2000b) formula for mixing above a circular source of buoyancy. For the miscible liquid-liquid (supernatant-non-convective layer) system of interest here, their formula takes the form

$$
\frac{\mathrm{T}_{\mathrm{CL}}-\mathrm{T}_{\mathrm{mix}}}{\mathrm{T}_{\mathrm{NCL}}-\mathrm{T}_{\mathrm{CL}}}=2.8\left[\frac{\mathrm{v}_{0}^{2}\left(\frac{\rho_{\mathrm{CL}}}{\rho_{\mathrm{NCL}}^{\prime}}\right)^{2}}{\mathrm{~g}\left(\frac{\rho_{\mathrm{CL}}}{\rho_{\mathrm{NCL}}^{\prime}}-1\right) \mathrm{R}_{0}}\right]^{1 / 3}
$$

where $\rho_{\mathrm{NCL}}^{\prime}$ is the two-phase density of the void-containing non-convective layer $\left(\rho_{\mathrm{CL}}>\rho_{\mathrm{NCL}}^{\prime}\right)$, $R_{0}$ is the effective horizontal extent of the mixing zone, and $v_{0}$ is the velocity (initial) of the buoyant parcel as it rises from and passes by the "surface" of the non-convective layer. Equation (4-20) is based on theoretical analysis and experimental data obtained specifically to address fluid mixing in Hanford waste tanks (Epstein and Burelbach 1998), and it is currently applied in the flammable gas safety basis (Slezak et al. 1998).

The rise velocity $v_{0}$ of the buoyant parcel is proportional to the density difference $\rho_{\mathrm{CL}}-\rho_{\mathrm{NCL}}^{\prime}$. The parcel cannot rise from the non-convective layer faster than the supernatant can flow into the region (cavity) vacated by the departing buoyant parcel. Thus, the velocity $v_{0}$ in equation (4-20) is less than or equal to the so-called exchange flow velocity $v_{0}$ across an opening of radius $\mathbf{R}_{0}$; namely (see Brown [1962] or Epstein [1988]),

$$
\mathrm{v}_{0}=0.1\left[\mathrm{R}_{0} \mathrm{~g}\left(\frac{\rho_{\mathrm{CL}}}{\rho_{\mathrm{NCL}}^{\prime}}-1\right)\right]^{1 / 2}
$$


Eliminating $v_{0}$ between equations (4-20) and (4-21) and assuming $\rho_{\mathrm{CL}} \square \rho_{\mathrm{NCL}}^{\prime}$ in the numerator of equation (4-20), gives the following simple result for the temperature of the mixing zone above the buoyant release area:

$$
\mathrm{T}_{\text {mix }}=\mathrm{T}_{\mathrm{CL}}+0.6\left(\mathrm{~T}_{\mathrm{NCL}}-\mathrm{T}_{\mathrm{CL}}\right)
$$

Inserting the typical values $\mathrm{T}_{\mathrm{NCL}}=118^{\circ} \mathrm{C}$ and $\mathrm{T}_{\mathrm{CL}}=100^{\circ} \mathrm{C}$ for the high self-heat tanks, gives $\mathrm{T}_{\text {mix }}=111^{\circ} \mathrm{C}$ or $\mathrm{T}_{\mathrm{NCL}}-\mathrm{T}_{\text {mix }}=7^{\circ} \mathrm{C}$. Such a large and sudden drop in temperature will collapse the steam voids within the initially buoyant parcel. The void collapse results in the withdrawal of the released material's buoyancy and the material settles back into the non-convective layer.

As the parcel slumps and spreads out over the top of the non-convective layer, its residual energy relative to the surrounding supernatant is transported upward through the supernatant to the surface where it is "absorbed" by surface evaporation (boiling). It is of interest to estimate the pressure rise in the headspace due to the upward energy flux from the spreading and cooling parcel. Heat flows from the parcel by turbulent natural convection in accord with equation (4-15):

$$
Q_{u}=k A\left(\frac{g \beta}{\alpha v}\right)^{\frac{1}{3}}(\Delta T)^{4 / 3}
$$

where $Q_{\mathfrak{u}}$ is the upward total heat flow, $A$ is the heat transfer area, and $\Delta T$ is the temperature difference between the parcel and the overlying supernatant. The maximum possible upward heat flow occurs from a parcel that spreads out over the entire non-convective layer, i.e., to the tank wall, so that $A=411 \mathrm{~m}^{2}$. The mixing calculation presented in the foregoing indicates that $\Delta \mathrm{T} ; 10^{\circ} \mathrm{C}$. Inserting these estimates into equation (4-23), together with the physical properties of water given below equation (4-15), yields a maximum upward heat flow $\mathrm{Q}_{\mathrm{u}}=1.12 \mathrm{MW}$. The volumetric flow of steam $\dot{V}_{v}$ at the surface of the supernatant in response to the upward heat flow is

$$
\dot{\mathrm{V}}_{\mathrm{v}}=\frac{\mathrm{Q}_{\mathrm{u}}}{\rho_{\mathrm{v}} \mathrm{h}_{\mathrm{fv}}}
$$

where $\rho_{v}$ is the density of saturated steam at one atmosphere $\left(0.6 \mathrm{~kg} \mathrm{~m}^{-3}\right)$. Assuming that the major resistance to steam flow from the tank headspace is exerted by the HEPA filters, the headspace pressure rise $\Delta \mathrm{P}$ above ambient required to accommodate the heat flow from the aborted steam bump is

$$
\Delta P=R \dot{V}_{v}=\frac{R Q_{u}}{\rho_{v} h_{f v}}
$$


where $\mathrm{R}$ is an empirical (filter) resistance coefficient equal to $2.34 \times 10^{3} \mathrm{~Pa} \mathrm{~s} \mathrm{~m}^{-3}$. The predicted pressure rise is $\Delta \mathrm{P}=2 \mathrm{kPa}(0.29 \mathrm{psi})$, which is negligible in comparison with the filter failure pressure of $36 \mathrm{kPa}$.

In summary, the buoyant displacement cannot be completed if the buoyant condition arises as a result of buildup of condensable vapor within the non-convective layer. A steam bump requires a sustained buoyant displacement, and this can only occur if noncondensable gas lifts a portion of the non-convective layer. Since noncondensable gas is not capable of performing this task in tanks 241-AZ-101, 241-AZ-102, and 241-AY-102, these tanks are not susceptible to steam bumps.

With regard to the historical bumping events, it is clear that the events occurred in tanks with deep supernatant layers and with sufficient powers to bring the non-convective layers to a boil and the supernatant layers up to their one-atmosphere boiling point. Many of the events were initiated by shutdown and restart of air lift circulators and may be classified as steam bumps by gas injection, as discussed in Section 4.1. Some of the events occurred naturally and periodically, most likely in tanks subject to episodic buoyant displacements driven by noncondensable gas generation. 
RPP-6213 REV 0

This page intentionally left blank.

FAI/00-14, Rev. 0 


\subsection{DESCRIPTION OF STEAM BUMP \& LIQUID WASTE RELEASE MODELS}

\subsection{STEAM BUMP SEQUENCE OF EVENTS}

In this section, we consider a hypothetical tank with a non-convective layer that is capable of self-heating to its boiling point and within which noncondensable gas generation may lead to a buoyant displacement event. The major assumption underlying the models presented in this section is that the near-boiling buoyant displacement parcel becomes fluid as soon as it starts to move. In Appendix C, the Meyer et al. (1997) energy criterion is used to justify this assumption.

The envisioned sequence of events that result in liquid waste release from a tank during a steam bump is illustrated in Figure 5-1. Gas released from the non-convective layer forms a cluster of fine bubbles at the bottom of the convective layer or supernatant (Figure 5-1a). The cluster rises and grows due to the growth of the numerous individual bubbles that comprise the cluster. The bubbles' demand for volume causes the liquid surface to rise and the headspace gas to compress (Figure 5-1b). If the waste surface rises above the location of an open vent, the combination of a high waste level relative to the elevation of the vent opening and a pressurized headspace causes liquid to flow from the tank to the outside. Steam bump model results show that this mode of waste release to the outside does not occur because the vent openings are located close to the tank dome. Ultimately, the bubbles reach the surface of the waste. As the bubbles break through the surface, the release of bubble gas and vapor to the headspace is accompanied by the ejection of a spray of liquid waste (Figure 5-1c). Simultaneous bubble breakthrough and aerosol generation are assumed to occur instantaneously at the waste surface and result in a spatially uniform aerosol concentration within the headspace (Figure 5-1d). Finally, the venting pressurized headspace gas carries waste aerosol to the vent where it is released to the outside (Figure 5-1e). The addition of steam to the headspace via bubble breakthrough causes the headspace to expand to a saturated state before the depressurization process is over. Consequently, fog formation occurs during depressurization which contributes to the aerosol loading within the headspace. Subsequent aerosol transport to the outside may occur via natural ventilation flow over a relatively long time scale compared with the duration of the depressurization stage of the steam bump (Figure 5-1e).

\subsection{BUBBLE AND WASTE ASCENT MODEL}

The heart of the steam bump model are the equations for the rate at which the gas bubbles grow as they rise through the convective layer. Recall that equations (4-7) and (4-12) describe the bubble-interior diffusion-limited growth of a representative bubble. They are rewritten below in terms of the bubble volume normalized by the initial bubble volume and time $t$; that is,

$$
\frac{d\left[P_{v} V_{b} / V_{b}(0)\right]}{d t}=\left[\frac{24 D U_{b}}{V_{b}(0)} \cdot \frac{V_{b}}{V_{b}(0)}\right]^{1 / 2} P_{\text {mix }} \ln \left[1+\frac{P_{e q}\left(T_{\ell}\right)-P_{v}}{P_{\text {mix }}-P_{e q}\left(T_{\ell}\right)}\right]
$$


Figure 5-1. Sequence of Events in Model of Steam Bump and Liquid Waste Release.
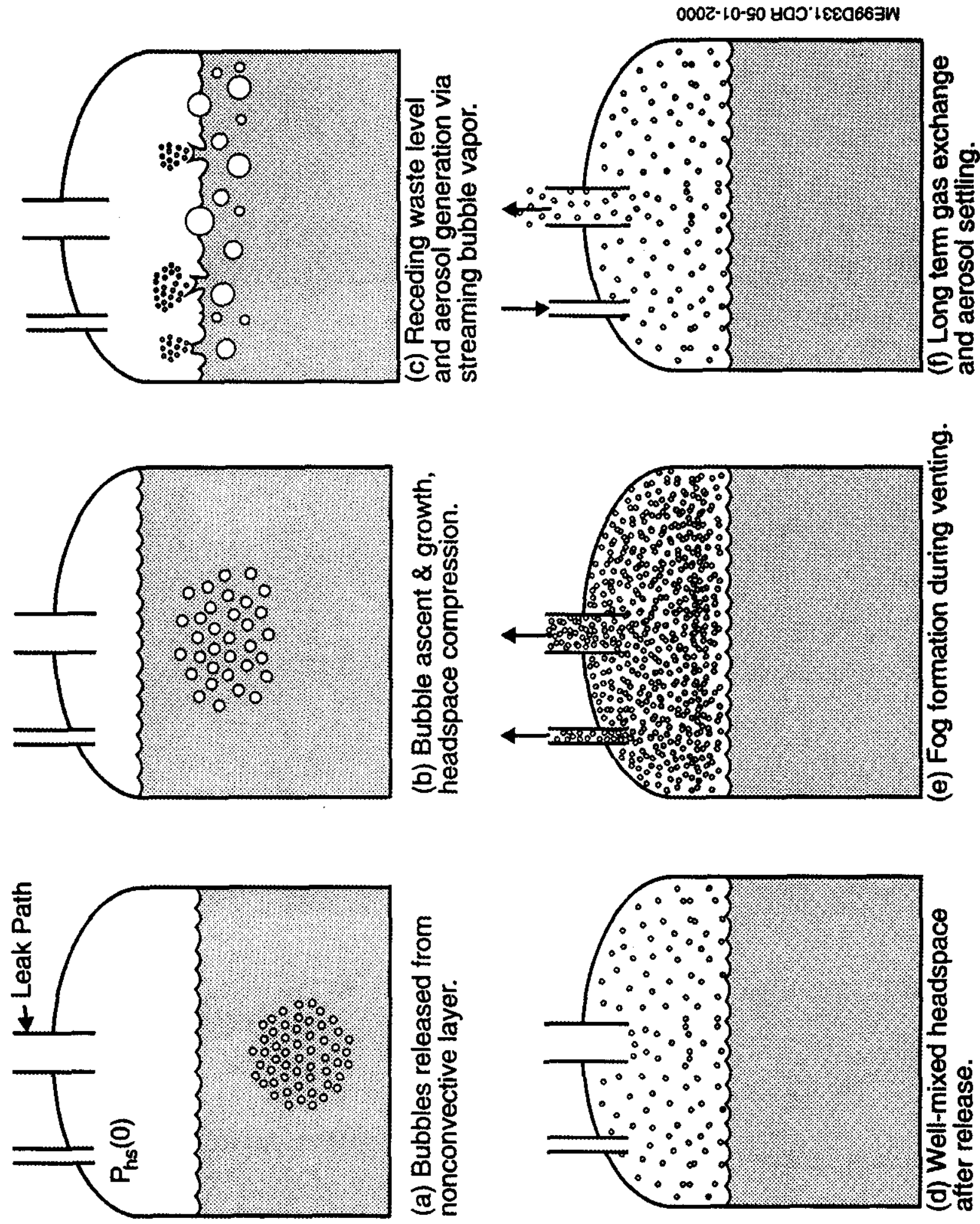


$$
\frac{\mathrm{V}_{\mathrm{b}}}{\mathrm{V}_{\mathrm{b}}(0)}=\frac{\mathrm{P}_{\mathrm{hs}}+\rho_{\ell} \mathrm{gH}-\mathrm{P}_{\mathrm{eq}}\left(\mathrm{T}_{\ell}\right)}{\mathrm{P}_{\text {mix }}-\mathrm{P}_{\mathrm{v}}}
$$

where $P_{\text {mix }}=P_{h s}+\rho_{\ell} g\left(H-z_{b c}\right)$

The symbol $V_{b}(0)$ in the group 24DU / $V_{b}(0)$ is the volume of a representative bubble. As already mentioned, equations (5-1) and (5-2) are valid for both an isolated bubble and a cluster of bubbles, i.e., $V_{b c}(z) / V_{b c}(0)=V_{b}(z) / V_{b}(0)$ where $V_{b c}$ is the bubble cluster volume and $V_{b c}(z) / V_{b c}(0)$ represents the volume expansion ratio of the bubble cluster at time $t$ or at location $z$ above the bottom of the convective layer (at $z=0$ ). The instantaneous location $z_{b c}$ of the center of the bubble cluster is related to time by the differential equation

$$
\frac{d z_{b c}}{d t}=U
$$

where $U$ is given by equation (4-13) for the rise velocity of a bubble cluster. In terms of the total volume of the bubbles in the cluster, this equation becomes:

$$
\mathrm{U}=0.76\left(\mathrm{~g}^{3} \mathrm{~V}_{\mathrm{bc}}\right)^{1 / 6}
$$

The value of $P_{e q}\left(T_{\ell}\right)$ in equations (5-1) and (5-2) depends on whether the bubbles are surrounded by supernatant liquid or, in the event of a buoyant displacement, by buoyant sludge (nonconvective) interstitial liquid. In the former case, $T_{\ell}$ is limited by the boiling point of the supernatant at essentially atmospheric pressure; while in the latter case, $T_{l}$ may exceed this limit. For the buoyant displacement case, $T_{l}$ is a time-varying function which is determined by the mixing process which takes place between the buoyant parcel and the surrounding supernatant. This mixing process is different than the mixing process discussed at the end of Section 4.2, which occurs via a vertical buoyancy-dominated turbulent diffusion mechanism just as the parcel tries to emerge from the non-convective layer. In what follows, the mixing process is driven by the momentum carried by the buoyant parcel after it separates completely from the nonconvective layer and while it rises to the surface of the waste.

To calculate the rate of mixing of the buoyant parcel with the supernatant, we adopt the nowclassical entrainment assumption (Morton et al. 1956) which states that the mean supernatant inflow (entrainment) velocity $v_{\text {en }}$ across the edge of the parcel is proportional to the instantaneous rise velocity $U$ of the parcel; that is,

$$
\mathrm{v}_{\mathrm{en}}=\mathrm{E}_{\mathrm{o}} \mathrm{U}
$$

where $E_{0}$ is the so-called entrainment coefficient with an experimentally determined value of approximately 0.1 . Equation (5-6) has proved enormously successful as an effective way of quantifying gas-phase and liquid-phase mixing problems involving jets, plumes, or buoyant puffs over a very wide range of scales (see, e.g., Briggs [1969] and Turner [1973]). 
The instantaneous liquid mass $m_{\ell}$ within the buoyant parcel may be determined from the continuity equation

$$
\frac{\mathrm{d} \mathrm{m}_{\ell}}{\mathrm{dt}}=\rho_{\ell} \mathrm{v}_{\mathrm{en}} A_{\mathrm{bp}}-\dot{\mathrm{m}}_{\mathrm{v}}
$$

where $\rho_{\ell}$ is the liquid density, $\dot{\mathrm{m}}_{\mathrm{v}}$ is the mass rate at which liquid is converted to vapor by bubble growth within the buoyant parcel, and $A_{b p}$ is the instantaneous area of the boundary through which supernatant is entrained by the buoyant parcel:

$$
A_{b p}=4.84\left(V_{b}+\frac{m_{\ell}}{\rho_{\ell}}\right)^{2 / 3}
$$

The instantaneous mean temperature $T_{\ell}$ of the liquid mass within the buoyant parcel is predicted with the energy equation

$$
\mathrm{m}_{\ell} \mathrm{c}_{\ell} \frac{\mathrm{d} \mathrm{T}_{\ell}}{\mathrm{dt}}=-\rho_{\ell} \mathrm{v}_{\mathrm{en}} A_{\mathrm{bp}} \mathrm{c}_{\ell}\left(\mathrm{T}_{\ell}-\mathrm{T}_{\mathrm{CL}}\right)-\dot{\mathrm{m}}_{\mathrm{v}} \mathrm{h}_{\mathrm{fv}}
$$

where $T_{C L}$ is the constant temperature of the non-convective layer and $c_{\ell}$ is the specific heat of the liquid. Note that in integrating equations (5-7) and (5-9), the mass of the solid component of the material released from the non-convective layer is included in the initial volume of $m_{\ell}$. For the sake of simplicity, the differences between the liquid and solid densities and specific heats are not included in equations (5-8) and (5-9).

The important initial conditions for the numerical simulation of a buoyant displacement-induced steam bump are the total volume $V_{b}(0)$ of the bubbles and the mass $m_{l}(0)$ of liquid that participates in the buoyant displacement. Meyer et al. (1997) recommend the following formula for the volume of gas released to the headspace during a buoyant displacement:

$$
V_{b}(0)=750 \frac{\alpha_{N B} h_{N C L} \tau_{y}}{P_{N C L} \rho_{C L}}
$$

where $\tau_{\mathrm{y}}$ is the yield stress of the non-convective material in $\mathrm{Pa}$ and $\alpha_{\mathrm{NB}}$ is the neutral buoyant void fraction. Equation (5-10) is an approximate result that Meyer et al. (1997) derived from a more cumbersome set of equations; it is a dimensional equation that requires the units $h_{\mathrm{NCL}}$ in $\mathrm{m}$, $\rho C L$ in $\mathrm{kg} \mathrm{m}^{-3}$, and $\mathrm{P}_{\mathrm{NCL}}$ in atm. Actually equation (5-10) is the Meyer et al. (1997) expression divided by $\left(\mathrm{P}_{\mathrm{NCL}}\right.$ - 1) $\mathrm{P}_{\mathrm{NCL}}$, since it is the volume of the gas in the just released buoyant parcel that is of interest here rather than the volume of gas released to the headspace. The leading coefficient in equation (5-10) was chosen to best match the gas release volumes from the tank buoyant displacement data. Equation (5-10) is applicable only to the relatively weak nonconvective layers in the double-shell tanks for which $\tau_{y} \sim 100 \mathrm{~Pa}$. Once $V_{b}(0)$ is determined from equation (5-10), the initial void-free mass of the buoyant parcel is estimated using 


$$
\mathrm{m}_{\ell}(0)=\rho_{\mathrm{NCL}} \mathrm{V}_{\mathrm{b}}(0)\left(\frac{1}{\alpha_{\mathrm{NB}}}-1\right)
$$

Another parameter of interest to tank bump consequence analysis is the frequency of a buoyant displacement event. The average time $t_{\mathrm{BD}}$ between any two buoyant displacement events can be shown to be (Meyer et al. 1997):

$$
t_{\mathrm{BD}}=\frac{V_{\mathrm{b}}(0)}{\mathrm{G}_{\mathrm{g}} \mathrm{A}_{\mathrm{T}} \mathrm{h}_{\mathrm{NCL}}}
$$

where $A_{T}$ is the tank cross-sectional area. Equation (5-12) is based on the Meyer et al. (1997) formulation for a right circular cylindrical displacement parcel; it may be derived as follows. Referring to the equation numbers in the Meyer et al. (1997) report, divide equation (4.5.11) by equation (4.5.3) and use equation (4.5.5) to eliminate the waste level rise rate $\mathrm{dh} / \mathrm{dt}$ from the result. Regardless of whether or not the non-convective layer is at its boiling point, $G_{g}$ in equation (5-12) is the volume of noncondensable gas generated per unit volume of nonconvective material per unit time, since the noncondensable gas is responsible for the buoyant displacement (see Section 4.2).

The headspace pressure $P_{h s}$ in equation (5-2) is as yet an unknown quantity. Early on, the dynamic interaction between cluster bubble growth and the simultaneous compression of the headspace atmosphere determines the instantaneous value of $\mathrm{P}_{\mathrm{hs}}$. Later, fog formation and open ventilation paths determine the value of $P_{\text {hs. }}$. The steam bump simulations are carried out by inserting equations (5-1) to (5-9) into the HADCRT waste tank source term computer model. The HADCRT model has been described by Malinovic et al. (2000) and will not be belabored here. Suffice it to say that the model is capable of tracking in-leakage and vent-path flows, simultaneous fog formation and deposition within the headspace, waste aerosol released from the headspace to the outside, and waste aerosol that enters the headspace from below. With respect to waste aerosol that enters the headspace from below, it remains to write the equations that describe the rate of generation of liquid aerosol by gas bubble breakthrough.

\subsection{AEROSOL RELEASE MODEL}

To simplify the calculation of droplet generation by the mechanism of gas bubble breakthrough at the liquid surface, all the bubbles are assumed to pass through the surface simultaneously and, correspondingly, the supernatant pool suddenly collapses to its initial depth $H(0)$. The volume of liquid waste aerosol $\mathrm{V}_{\mathrm{a}}$ that enters the headspace air (hereafter referred to as entrained liquid) is given by the definition of the entrainment coefficient E (see e.g., Ginsberg [1983]; or Kataoka and Ishii [1984]):

$$
\mathrm{V}_{\mathrm{a}}=\mathrm{E}\left(\frac{\rho_{\mathrm{hs}}}{\rho_{\ell}}\right) \mathrm{V}_{\mathrm{b}}(\mathrm{H})
$$


where $\rho_{h s}$ is the density of the compressed headspace air and $\mathrm{V}_{\mathrm{b}}(\mathrm{H})$ is the volume of the bubble cluster, both evaluated at the instant the cluster arrives at the liquid surface, that is when $z_{b c}=H$. The mass $m_{a}$ of aerosol entrained is

$$
\mathrm{m}_{\mathrm{a}}=\mathrm{V}_{\mathrm{a}} \rho_{\mathrm{l}}=\mathrm{E} \rho_{\mathrm{hs}} \mathrm{V}_{\mathrm{b}}(\mathrm{H})
$$

The droplets produced just above the waste surface will not all be carried off into the core of the headspace atmosphere. Only those droplets whose terminal velocities are less than the gas velocity generated by bubble break through will be carried off. Kataoka and Ishii (1984) have developed the following correlation of the data of Golub (1970) and Garner et al. (1954) for the efficiency of stable aerosol production above a liquid pool with gas sparging:

$$
E=7.13 \times 10^{-4}\left[\frac{\mu_{g}^{4} \rho_{g}^{2}\left(\rho_{\ell}-\rho_{g}\right)^{3}}{\sigma^{9} g^{5}}\right]^{1 / 8} j_{g}^{3}
$$

where $\mathrm{j}_{\mathrm{g}}$ is the superficial velocity of the sparging gas, $\mu_{\mathrm{g}}$ and $\rho_{\mathrm{g}}$ are the viscosity and density of the bubble gas, respectively, and $\sigma$ is the interfacial tension of the waste liquid. For the present application, $\mathrm{j}_{\mathrm{g}}$ must be related to the velocity $\mathrm{U}(\mathrm{H})$ of the bubble cluster when it arrives at the waste surface. The void fraction $\alpha$ of bubble clusters, estimated from slow motion photography (Marble 1983), appears to be about 0.5. Moody (1986) opines that a better estimate is $\alpha=2 / 3$, a value which is more consistent with bubble cluster rise velocities. It follows that

$$
\mathrm{j}_{\mathrm{g}}=\alpha \mathrm{U}(\mathrm{H})=\frac{2}{3} \mathrm{U}(\mathrm{H})
$$

Once the bubble cluster velocity $\mathrm{U}(\mathrm{H})$ at the waste surface is calculated, the mass of the entrained liquid can be determined from equations $(5-14)$ to $(5-16)$. This is the theoretical maximum quantity that can enter the environment.

A portion of the entrained liquid (aerosol) will escape from the tank early on by flow of pressurized headspace air through vents and leakage paths. Subsequently, over a much longer time span, a portion of the remaining aerosol may be released by free-convection-driven exchange flow between the outside and the tank headspace. If unfiltered leak paths are small or have high flow resistance, substantial aerosol release can only occur if the headspace pressure exceeds the HEPA filter failure pressure. The major results of the steam bump simulations are postponed until Section 8.0. However, it is useful at this point to discuss the predicted buoyant parcel temperature versus time trends because of their potential importance to aerosol production at the waste surface.

\subsection{EXAMPLE CALCULATION}

Due to the mixing of the buoyant parcel with the supernatant, the liquid component of the parcel is predicted to remain sub-cooled during its rise to the surface of the waste. Figure 5-2 shows the calculated temperature history (solid curve) of the parcel for a buoyant release of initial liquid 
Figure 5-2. Temperature History of Buoyant Parcel Compared With its Boiling Temperature; Open Headspace.

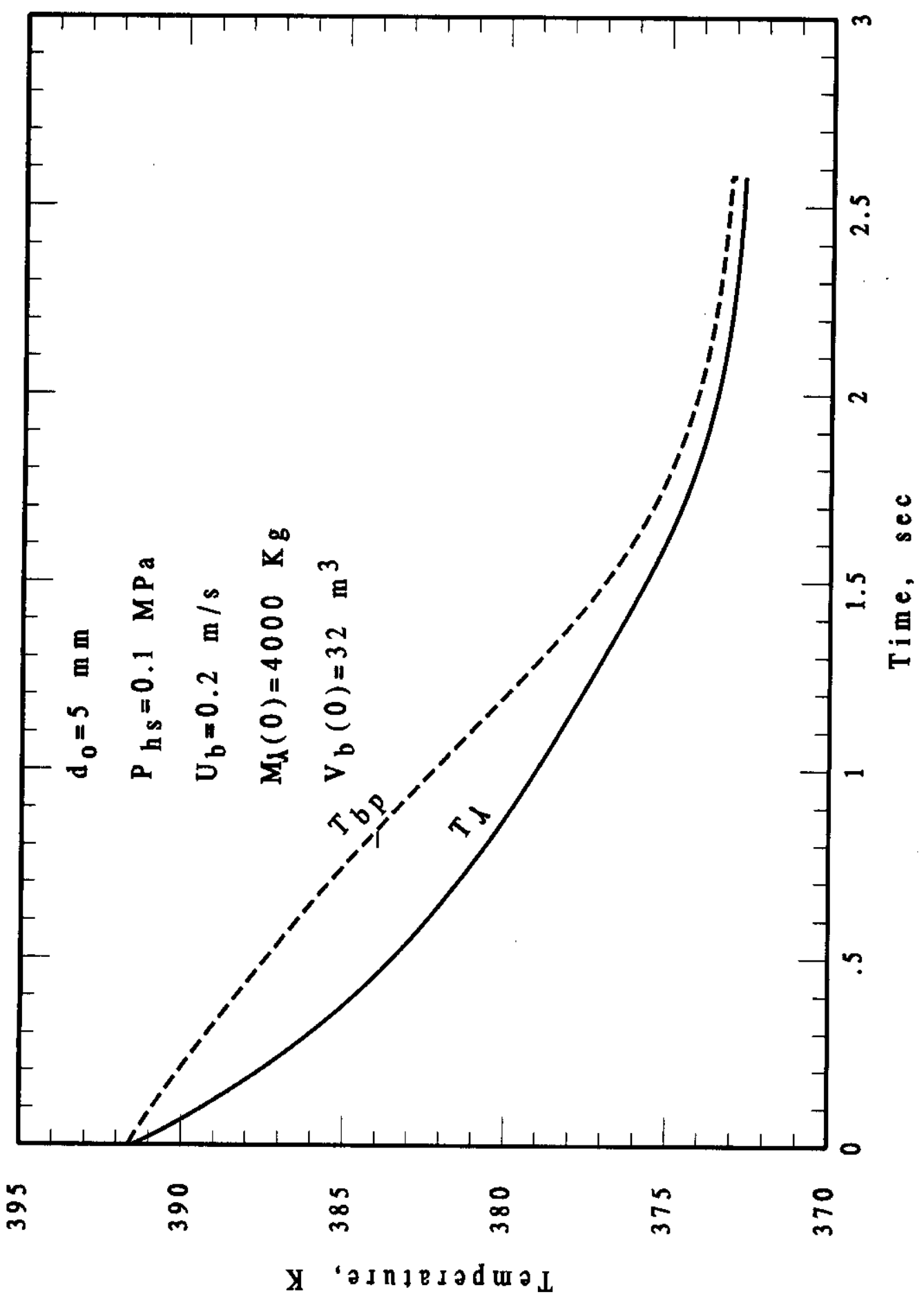


mass $4000 \mathrm{~kg}$, initial void volume $32 \mathrm{~m}^{3}$, and initial near-saturation temperature $391.5 \mathrm{~K}$ into an 8.0-m deep supernatant at $373 \mathrm{~K}$ and density $1210 \mathrm{~kg} \mathrm{~m}^{-3}$. The headspace pressure is constant and equal to atmospheric pressure. That is, the tank is assumed to be wide open to the outside. The dashed curve in Figure 5-2 represents the boiling point of the liquid in the buoyant parcel at the instantaneous location of the parcel. Clearly, since the boiling point always exceeds the parcel temperature, the only mechanism of vapor generation is evaporation at the surface of the bubbles that were originally trapped in the non-convective layer.

Suppose that the vessel remains effectively sealed during the period of buoyant parcel rise, and the temperature of the supernatant is assumed to be at its one-atmosphere boiling point. In this case, the liquid component of the parcel is predicted to be slightly superheated by several degrees relative to the atmospheric boiling point $373 \mathrm{~K}$ when the parcel reaches the surface, but subcooled relative to the boiling point at the peak tank pressure (see Figure 5-3). As the tank gradually depressurizes, the warm parcel liquid will spread out beneath the surface and ultimately it will begin to boil and produce steam at a rate dictated by the depressurization rate of the tank. Additional mixing may occur between the parcel liquid and the supernatant as the parcel spreads. Even if no credit is given for the additional mixing and an adiabatic evaporation process is assumed for the stratified parcel liquid, the steam flux across the waste surface is found to be small $\left(<0.1 \mathrm{~ms}^{-1}\right)$. Thus, the liquid aerosol above the waste surface is created by the busting of bubbles and the volume of the aerosol is found to be small compared with that of the aerosol produced earlier by the breakthrough of the bubbles transported to the free surface within the buoyant parcel.

\subsection{NUMERICAL EVALUATION NOTE}

To couple integration of bubble volume and vapor pressure with other quantities, the $P_{v}$ term of equation (5-1) is expanded as follows. State variables describing bubble rise are the bubble cluster volume expansion ratio $v=V_{b c}(z) / V_{b c}(0)$ and vapor pressure in the bubble $P_{v}$ which appear in equations $(5-1)$ and (5-2), are recast as:

$$
\begin{aligned}
& P_{v} \frac{d v}{d t}+v \frac{d P_{v}}{d t}=\sqrt{\frac{24 D U_{b} v}{V_{b 0}}} P_{\text {mix }} \ln \left[1+\frac{P_{e q}\left(T_{\ell}\right)-P_{v}}{P_{\text {mix }}-P_{e q}\left(T_{\ell}\right)}\right] \\
& \frac{d v}{d t}=\frac{d}{d t}\left[\frac{P_{h s}+\rho_{\ell} g H-P_{e q}}{P_{h s}+\rho_{\ell} g(H-z)-P_{v}}\right] \\
& \frac{\mathrm{d} v}{\mathrm{dt}}=\left(\frac{1-\mathrm{v}}{\Delta \mathrm{P}_{\mathrm{g}}}\right) \frac{\mathrm{d} \mathrm{P}_{\mathrm{hs}}}{\mathrm{dt}}+\rho_{\ell} \mathrm{g}\left(\frac{1-\mathrm{v}}{\Delta \mathrm{P}_{\mathrm{g}}}\right) \frac{\mathrm{dH}}{\mathrm{dt}}+\frac{\rho_{\ell} \mathrm{g} \mathrm{vU}}{\Delta \mathrm{P}_{\mathrm{g}}}+\frac{\mathrm{v}}{\Delta \mathrm{P}_{\mathrm{g}}} \frac{\mathrm{d} \mathrm{P}_{\mathrm{v}}}{\mathrm{dt}}
\end{aligned}
$$


Figure 5-3. Temperature History of Buoyant Parcel Compared With its Boiling Temperature; Sealed Headspace.

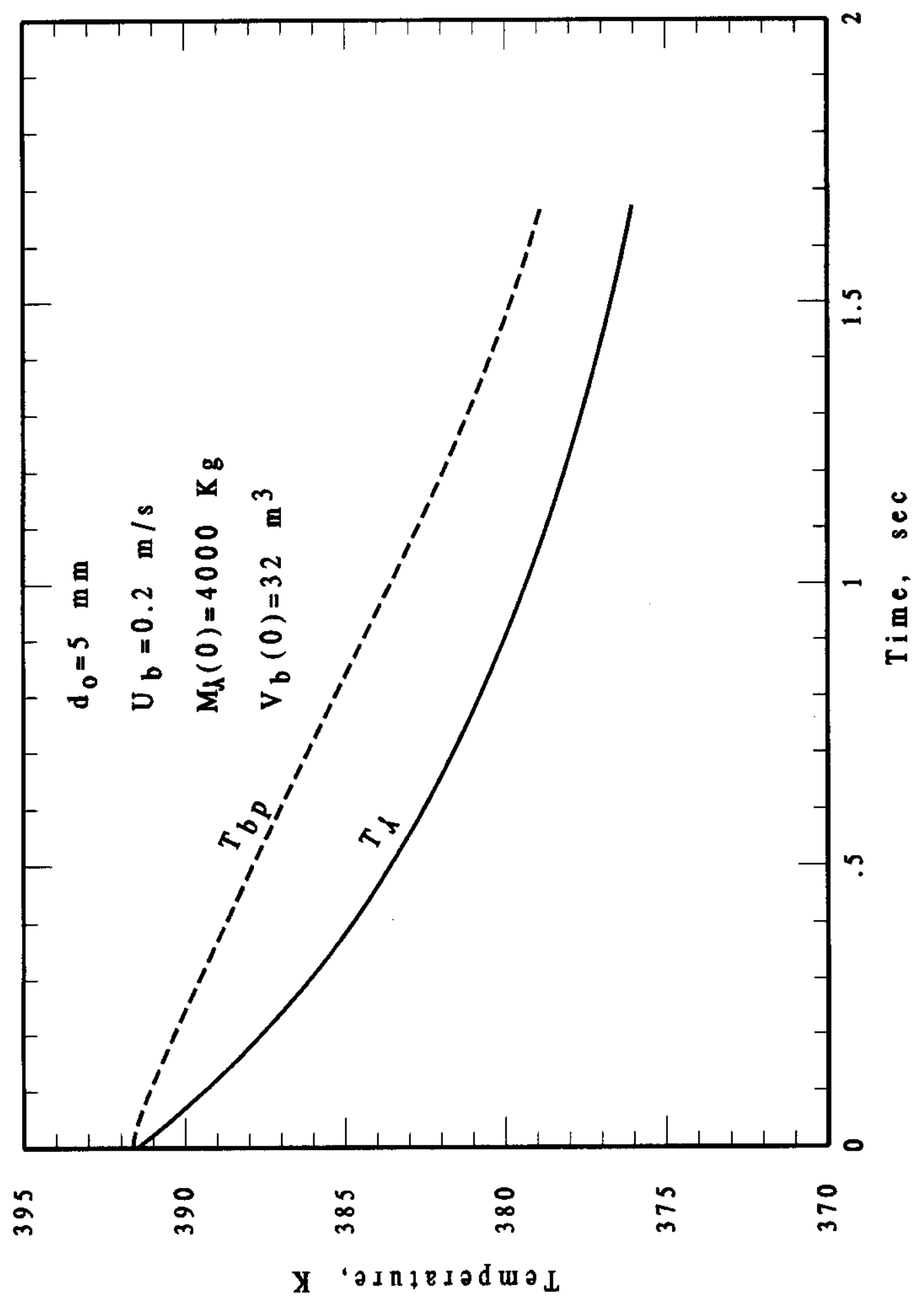


Derivatives other than $\mathrm{dP}_{\mathrm{v}} / \mathrm{dt}$ in equation (5-18) may be eliminated through auxiliary relations as follows. The headspace pressure derivative is given by

$$
\frac{d P_{h s}}{d t}=\frac{P_{h s}}{m_{h s}} \frac{d m_{h s}}{d t}-\frac{P_{h s}}{V_{h s}} \frac{d V_{h s}}{d t}+\frac{P_{h s}}{T_{h s}} \frac{d T_{h s}}{d t}
$$

The mass flowrate leaving the headspace is comprised of HEPA and leakage flow paths that can be quantified later:

$$
\begin{aligned}
& \frac{d m_{h s}}{d t}=-W_{h s} \\
& W_{h s}=W_{\text {HEPA }}+W_{\text {leak }} \\
& W_{\text {HEPA }}=\rho_{h s}\left(\frac{P_{h s}-P_{\text {amb }}}{R_{\text {HEPA }}}\right)
\end{aligned}
$$

The HEPA flowrate is directly proportional to pressure difference, and the standard compressible flow relation is used for leakage paths or in case of HEPA failure. The headspace volume derivative is simply

$$
\frac{d V_{h s}}{d t}=-V_{b c o} \frac{d v}{d t}
$$

The headspace temperature derivative is found from the energy equation

$$
\begin{aligned}
& m_{h s} c_{v h s} \frac{d T_{h s}}{d t}=-W_{h s}\left(c_{P h s}-c_{v h s}\right) T_{h s}+P_{h s} V_{b c o} \frac{d v}{d t} \\
& \frac{d T_{h s}}{d t}=-(\gamma-1) T_{h s} \frac{W_{h s}}{m_{h s}}+(\gamma-1) T_{h s} \frac{d v}{d t}
\end{aligned}
$$

which allows the pressure derivative to be recast as

$$
\frac{d P_{h s}}{d t}=-\frac{\gamma W_{h s} P_{h s}}{m_{h s}}+\frac{\gamma P_{h s} V_{b c o}}{V_{h s}} \frac{d v}{d t}
$$

Remaining derivatives required by equation (5-18) are

$$
\frac{\mathrm{d} z}{\mathrm{dt}}=\mathrm{U}
$$




$$
\frac{\mathrm{dH}}{\mathrm{dt}}=\mathrm{H}_{\mathrm{o}} \frac{\mathrm{V}_{\mathrm{bco}}}{\mathrm{V}_{\ell}+\mathrm{V}_{\mathrm{bco}}} \frac{\mathrm{d} \mathrm{v}}{\mathrm{dt}}
$$

when equations (5-18) through (5-24) are combined, this yields an equation of the form

$$
\begin{aligned}
& A_{12} \frac{d v}{d t}-\frac{v}{\Delta P_{g}} \frac{d P_{v}}{d t}=b_{2} \\
& A_{12}=1-\frac{1-v}{\Delta P_{g}}\left[P_{h s} \gamma_{h s} \frac{V_{b c o}}{V_{h s}}+\rho_{\ell} g H_{o} \frac{V_{b c o}}{V_{b c o}+V_{\ell}}\right] \\
& b_{2}=\frac{\rho_{\ell} g U v}{\Delta P_{g}}-\left(\frac{1-v}{\Delta P_{g}}\right) \gamma_{h s} P_{h s} \frac{W_{h s}}{M_{h s}}
\end{aligned}
$$

so that equations (5-17) and (5-25) may be solved simultaneously to calculate bubble cluster rise. 
RPP-6213 REV 0

This page intentionally left blank. 


\subsection{BUMP CRITERIA MODEL APPLICATION}

Current safe-storage conditions are first considered to find a short list of tanks that would be susceptible to bumps after a prolonged duration without active heat removal. This consideration is repeated assuming that there are no ventilation system controls. In practice, this means that initial conditions for waste temperature are the limiting conditions for operation (LCO) rather than the current waste temperatures. The criteria developed in the previous sections are then applied to rationalize historic events shown in Table 3-1. Note that the criteria may be continually re-applied when reference tank conditions change.

\subsection{APPLICATION OF TANK BUMP CRITERIA TO CURRENT TANK WASTE}

This section applies the criteria developed in Section 4.0 to the Hanford SSTs and DSTs under a safe-storage off-normal scenario. This scenario assumes that the waste is undisturbed but there is no active system (ALC, primary system ventilation or annular ventilation system) for heat removal.

Criteria are presented and ordered to create a graded approach from the easiest to most difficult to apply, and to leave successively fewer remaining tanks to screen. Individual tanks are excluded from further consideration if any of the following are true:

1. There is an insignificant non-convective layer,

2. Supernatant depth does not exceed $1 \mathrm{~m}$,

3. Total tank heat load can be removed by steady-state conduction through the soil overburden (total tank heat load is less than $8500 \mathrm{~W}$ ),

4. The time to saturated tank conditions exceeds the time needed for ventilation system repairs using a lumped capacitance (single waste temperature) approach,

5. The time to saturated tank conditions exceeds the time needed for ventilation system repairs using separate energy balances for supernatant and non-convective layers, and

6. Neutral buoyancy cannot be attained in the non-convective layer through buildup of noncondensible gases.

The first two criteria are obvious from the discussion in Sections 3.0 and 4.0. The third criterion is derived from the one-dimensional, steady-state conduction equation:

$$
\dot{\mathrm{Q}}=\frac{\mathrm{kA} \Delta \mathrm{T}}{\delta}
$$


where $\dot{Q}$ is the total heat removal in $\mathrm{W}, \mathrm{k}$ is the soil thermal conductivity $(1.0 \mathrm{~W} / \mathrm{m}-\mathrm{K}), \mathrm{A}$ is the heat transfer area $\left(411 \mathrm{~m}^{2}\right), \Delta \mathrm{T}$ is the difference between the maximum allowable tank dome temperature and the Hanford environment average temperature, and $\delta$ is the soil overburden, which has a nominal thickness of $4.0 \mathrm{~m}$ (Kummerer 1994). Assuming that the supernatant temperature and dome temperature are the same, the maximum allowable temperature is just a few degrees below the boiling point. With a site average temperature of $14^{\circ} \mathrm{C}$ (Kummerer 1994), $\Delta \mathrm{T} \approx 80^{\circ} \mathrm{C}$ is justifiable. These assumptions result in $\dot{Q}=8500 \mathrm{~W}$ after rounding down. This calculation conservatively ignores downward or sideways conduction, and yields an easily applied screening criterion.

Considering SSTs, all but the four tanks listed in Table 3-5 fail criterion \#2, and are excluded on that basis. Table 6-1 lists DSTs with trivial non-convective layer depths, while Table 6-2 lists tanks that are excluded from further consideration based on small heat load $(<8500 \mathrm{~kW})$ and/or small supernatant depth. SSTs 241-A-101 and 241-AX-101 are evaluated here as if they have the waste configuration for a tank bump. Tanks not on either list are next evaluated for the time needed to reach saturated conditions, criteria 4 and 5 , in that order.

Table 6-1. DSTs With Negligible Non-Convective Layer (Hu et al. 2000).

\begin{tabular}{|c|}
\hline $241-A P-101$ \\
\hline $241-A P-102$ \\
\hline $241-A P-103$ \\
\hline $241-A P-104$ \\
\hline $241-A P-106$ \\
\hline $241-A P-107$ \\
\hline $241-A P-108$ \\
\hline
\end{tabular}

Table 6-2. SSTs and DSTs With Small Heat Load and/or Small Supernatant Depth (Hu et al. 2000).

\begin{tabular}{|c|c|c|c|c|}
\hline Tank & $\begin{array}{c}\text { Waste Depth } \\
\text { (m) }\end{array}$ & $\begin{array}{l}\text { Non-Convective } \\
\text { Layer Depth (m) }\end{array}$ & $\begin{array}{c}\text { Supernatant Depth } \\
(\mathrm{m})\end{array}$ & $\begin{array}{l}\text { Heat Load } \\
\text { (W) }\end{array}$ \\
\hline \multicolumn{5}{|c|}{ SSTS } \\
\hline 241-A-101 & 8.78 & 4.10 & 4.67 & 7340 \\
\hline 241-AX-101 & 6.90 & 3.34 & 3.56 & 5890 \\
\hline 241-S-111 & 4.98 & 3.96 & 1.02 & 6990 \\
\hline 241-SX-102 & 4.74 & 3.51 & 1.24 & 5020 \\
\hline
\end{tabular}




\begin{tabular}{|c|c|c|c|c|}
\hline Tank & $\begin{array}{c}\text { Waste Depth } \\
(\mathbf{m})\end{array}$ & $\begin{array}{c}\text { Non-Convective } \\
\text { Layer Depth }(\mathbf{m})\end{array}$ & $\begin{array}{c}\text { Supernatant Depth } \\
(\mathbf{m})\end{array}$ & $\begin{array}{c}\text { Heat Load } \\
(\mathbf{W})\end{array}$ \\
\hline 241-AN-101 & 1.48 & 0.30 & 1.17 & 2790 \\
\hline 241-AN-106 & 0.36 & 0.16 & 0.20 & 138 \\
\hline 241-AP-105 & 7.06 & 0.82 & 6.24 & 2780 \\
\hline 241-AW-102 & 0.74 & 0.37 & 0.37 & 1530 \\
\hline 241-AW-103 & 4.70 & 3.21 & 1.49 & 674 \\
\hline 241-AW-104 & 10.32 & 2.13 & 8.19 & 2530 \\
\hline 241-AW-105 & 3.96 & 2.58 & 1.37 & 827 \\
\hline 241-AW-106 & 4.36 & 2.10 & 2.26 & 2800 \\
\hline 241-AY-101 & 1.42 & 1.00 & 0.42 & 14400 \\
\hline 241-SY-102 & 5.64 & 0.81 & 4.83 & 1580 \\
\hline 241-SY-103 & 6.86 & 3.34 & 3.53 & 4760 \\
\hline
\end{tabular}

Note that tanks 241-AN-106 and 241-AW-102 fail on the account of supernatant depth and total heat load (criterion \#3). From an operational standpoint, these DSTs can be excluded on the basis of total tank power alone, which means that variable supernatant level is not a concern. Tank 241-AY-101 cannot be excluded on the basis of criterion \#3, but as shown below, a DST with $14.4 \mathrm{~kW}$ would take so long to self-heat to bump conditions that $241-\mathrm{AY}-101$ can also be excluded on criterion \#4 if need be.

The time to saturated conditions can be estimated using a lumped capacitance solution for the transient waste temperature:

$$
\left(V_{n c} \rho_{n c} c_{n c}+V_{c l} \rho_{c l} c_{c l}\right) \frac{d T}{d t}=\dot{Q}-\frac{k_{s} A\left(T-T_{\infty}\right)}{\delta}-\frac{k_{s} A\left(T-T_{\infty}\right)}{R}
$$

where $\mathrm{V}, \rho$, and $\mathrm{c}$ are volume, density and specific heat, respectively, subscript nc denotes the non-convective layer, subscript cl denotes convective layer, $\mathrm{T}$ is the waste average temperature, $\dot{Q}$ is the tank heat load, $k_{s}$ is soil thermal conductivity $(1.0 \mathrm{~W} / \mathrm{m}-\mathrm{K}), \mathbf{A}$ is the tank heat transfer area $\left(411 \mathrm{~m}^{2}\right), \delta$ is the soil thickness $(4 \mathrm{~m}), R$ is the tank radius $(11 \mathrm{~m})$, and $T_{\infty}$ is the average ambient temperature of $14^{\circ} \mathrm{C}$. Solving this first-order, linear differential equation gives the time to saturated conditions, $t_{b p}$, subject to the initial condition that $T=T_{0}$ :

$$
\mathrm{t}_{\mathrm{bp}}=\frac{1}{\mathrm{~K}} \ln \left[\frac{\mathrm{T}_{\infty}+\dot{\mathrm{q}} / \mathrm{K}-\mathrm{T}_{0}}{\mathrm{~T}_{\infty}+\dot{\mathrm{q}} / \mathrm{K}-\mathrm{T}_{\mathrm{bp}}}\right]
$$


where $\dot{\mathrm{q}}=\frac{\dot{\mathrm{Q}}}{\mathrm{V}_{\mathrm{nc}} \rho_{\mathrm{nc}} \mathrm{c}_{\mathrm{nc}}+\mathrm{V}_{\mathrm{cl}} \rho_{\mathrm{cl}} \mathrm{c}_{\mathrm{cl}}}$

and $\quad K=\frac{k_{s} A\left(\frac{1}{\delta}+\frac{1}{R}\right)}{V_{n c} \rho_{n c} c_{n c}+V_{c l} \rho_{c l} c_{c l}}$

Initial temperature $T_{0}$ is assumed to be the peak non-convective layer temperature, which conservatively overstates the initial waste average temperature. Final temperature, $T_{b p}$, is the supernatant boiling point, accounting for vapor suppression by dissolved salts. Tank-specific data is not available for supernatant boiling point, but a convenient expression stating that the supernatant vapor pressure is $85 \%$ of the vapor pressure of pure water is used instead (Crea et al. 2000). Supernatant boiling point is then $104^{\circ} \mathrm{C}$.

The method described here updates Kummerer's (1994) calculation by using recent tank-specific data and incorporating approximate expressions for conduction losses. The approximate expression for heat loss through the boil overburden is simply the steady-state expression for one-dimensional conduction through a planar slab held at the average waste temperature, $T$, on one side, and the average ambient air temperature, $\mathrm{T}_{\infty}$, on the other side. The expression for heat loss to underlying soil is the steady-state solution to the conduction equation for a circular region of radius $R$, at temperature $T$, in contact with a semi-infinite medium at temperature, $T_{\infty}$. The closed form solution assumes that the average air temperature and soil temperature are equal, when they actually differ by a degree $\mathrm{C}$ or so. A quasi-steady state formulation is an excellent approximation for times greater than the time constant for conduction, which is 106 days $\left(\mathrm{R}^{2} / \pi \alpha=10^{8}\right.$ seconds) for the underlying soil.

Table 6-3 shows the results of this calculation for DSTs that have not been screened already (i.e., not appearing in Tables 6-1 nor 6-2), but excluding the AZ / AY tank farm. In Table 6-3, initial temperature and one set of tank heat load inputs are from the Barton and Bingham (1999) database; the other set of tank heat load inputs is from $\mathrm{Hu}$ et al. (2000). Excluding the AZ and AY DSTs, the tank heat loads shown in Hu et al. (2000) are smaller than those in the Barton and Bingham (1999) database. Clearly, the calculated DST time to saturated condition values greatly exceed any of the corrective maintenance times mentioned in Section 3.5.3. An "Inf." entry means that the DST reaches a steady-state condition below $\mathrm{T}_{\mathrm{bp}}$.

AZ and AY DSTs require a more detailed calculation because there is a significant temperature difference between the convective and non-convective layers. An energy balance can be written for each layer:

$$
\begin{aligned}
& \rho_{\mathrm{CL}} \mathrm{V}_{\mathrm{CL}} \mathrm{c}_{\mathrm{CL}} \frac{\mathrm{d} \mathrm{T}_{\mathrm{CL}}}{\mathrm{dt}}=\dot{\mathrm{Q}}_{\mathrm{CL}}-\frac{\mathrm{k}_{\mathrm{s}} \mathrm{A}}{\delta}\left(\mathrm{T}_{\mathrm{CL}}-\mathrm{T}_{\infty}\right)+\mathrm{h}_{\mathrm{ex}} \mathrm{A}\left(\mathrm{T}_{\mathrm{NCL}}-\mathrm{T}_{\mathrm{CL}}\right) \\
& \rho_{\mathrm{NCL}} \mathrm{V}_{\mathrm{NCL}} \mathrm{c}_{\mathrm{NCL}} \frac{\mathrm{d} \mathrm{T}_{\mathrm{NCL}}}{\mathrm{dt}}=\dot{\mathrm{Q}}_{\mathrm{NCL}}-\frac{\mathrm{k}_{\mathrm{s}} \mathrm{A}}{\mathrm{R}}\left(\mathrm{T}_{\mathrm{NCL}}-\mathrm{T}_{\mathrm{s}}\right)-\mathrm{h}_{\mathrm{ex}} A\left(\mathrm{~T}_{\mathrm{NCL}}-\mathrm{T}_{\mathrm{CL}}\right)
\end{aligned}
$$


Table 6-3. Time to Saturated Conditions for DSTs With Significant Supernatant Depth \& Heat Load.

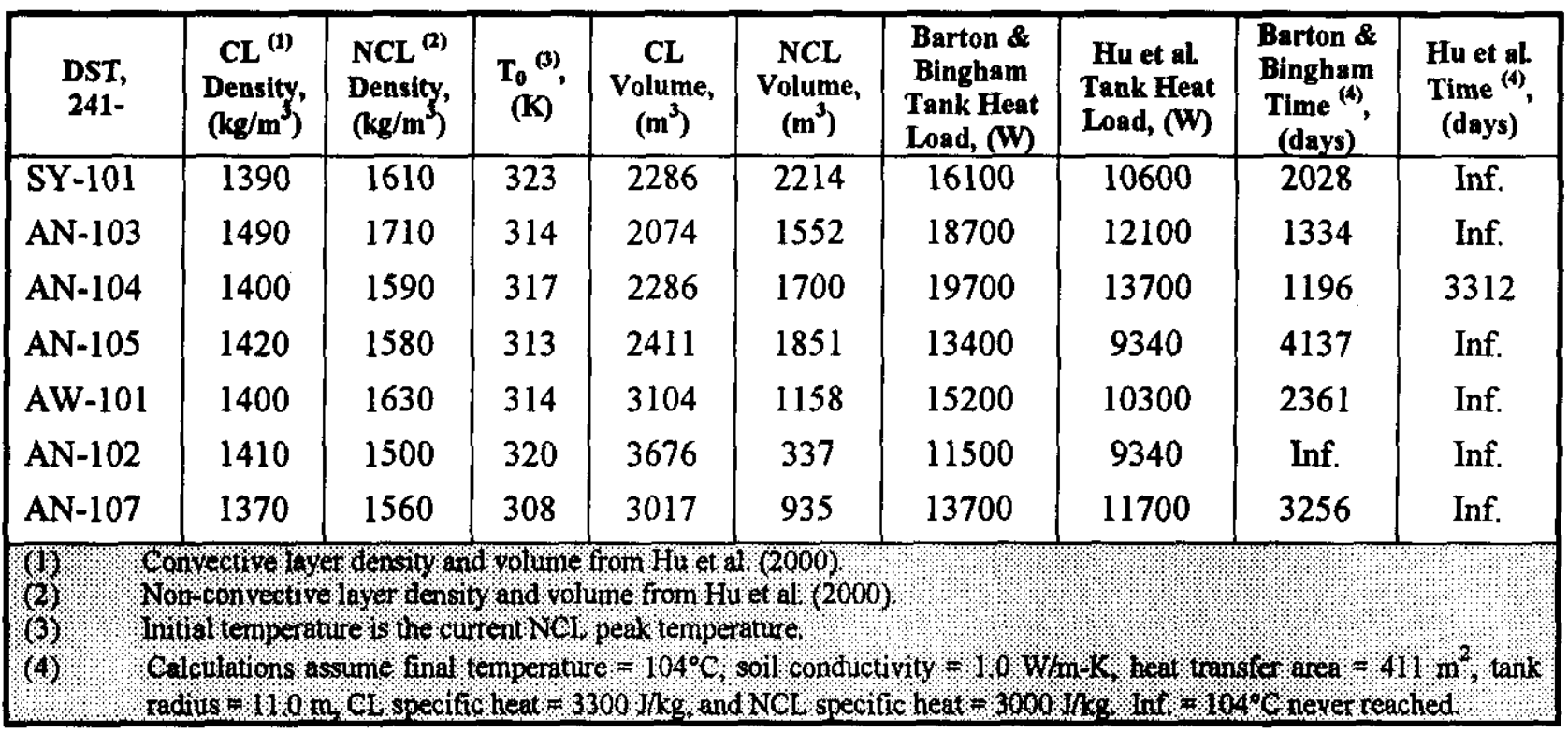

where symbols and subscripts are defined as above. A heat transfer coefficient, $h_{e x}$, is defined for exchange between the two layers. This heat transfer coefficient is sized so that in the quasisteady approximation,

$$
\frac{\mathrm{dT}_{\mathrm{CL}}}{\mathrm{dt}}=\frac{\mathrm{dT}_{\mathrm{NCL}}}{\mathrm{dt}}
$$

For DSTs 241-AZ-101 and -102, this system of equations was integrated under the initial condition that the supernatant temperature is the same as the headspace temperature, which is available from the Barton and Bingham (1999) database. Initial temperature, $\mathbf{T}_{\mathbf{N C L}}$, was assumed to be the peak non-convective layer temperature. Total heat loads are based on the Bingham and Barton (1999) database and the heat load split between convective and nonconvective layers is defined (Crea et al. 2000). Bump conditions exist when the supernatant reaches the boiling point $\left(104^{\circ} \mathrm{C}\right.$, as described above) or the non-convective layer reaches saturated conditions at the non-convective layer mid-height. Accounting for vapor suppression by dissolved salts, boiling point at the non-convective layer mid-height would be 398 and $396 \mathrm{~K}$, respectively, for tanks 241-AZ-101 and 241-AZ-102.

For tanks 241-AZ-101 and 241-AZ-102, calculation inputs and the time to bump are listed in Table 6-4. Figure 6-1 shows temperature histories for the 241-AZ-101 non-convective and supernatant layers. At 111 days, the supernatant temperature is just $104^{\circ} \mathrm{C}$ and the nonconvective layer is approaching $398 \mathrm{~K}$. Figure 6-2 shows that for 241-AZ-102, the nonconvective layer reaches the limiting temperature of $396 \mathrm{~K}$ at 118 days, at which time the supernatant temperature is still well below $377 \mathrm{~K}$. If the $\mathrm{Hu}$ et al. (2000) heat loads are considered, the time to bump values reported in Table 6-4 will decrease. Hu et al. (2000) report a total tank head load of $91,200 \mathrm{~W}$ for 241-AZ-101 and 79,800 for 241-AZ-102. Because the heat load is the dominant term in either energy balance (equations (6-6) and (6-7)), the time to bump is proportional to the inverse of tank heat load. A good estimate is that the 241-AZ-101 


\section{RPP-6213 REV 0}

Figure 6-1. 241-AZ-101 Heatup After Loss of Ventilation for Current Conditions.

\section{AZ-101 Heatup After Loss of Ventilation}

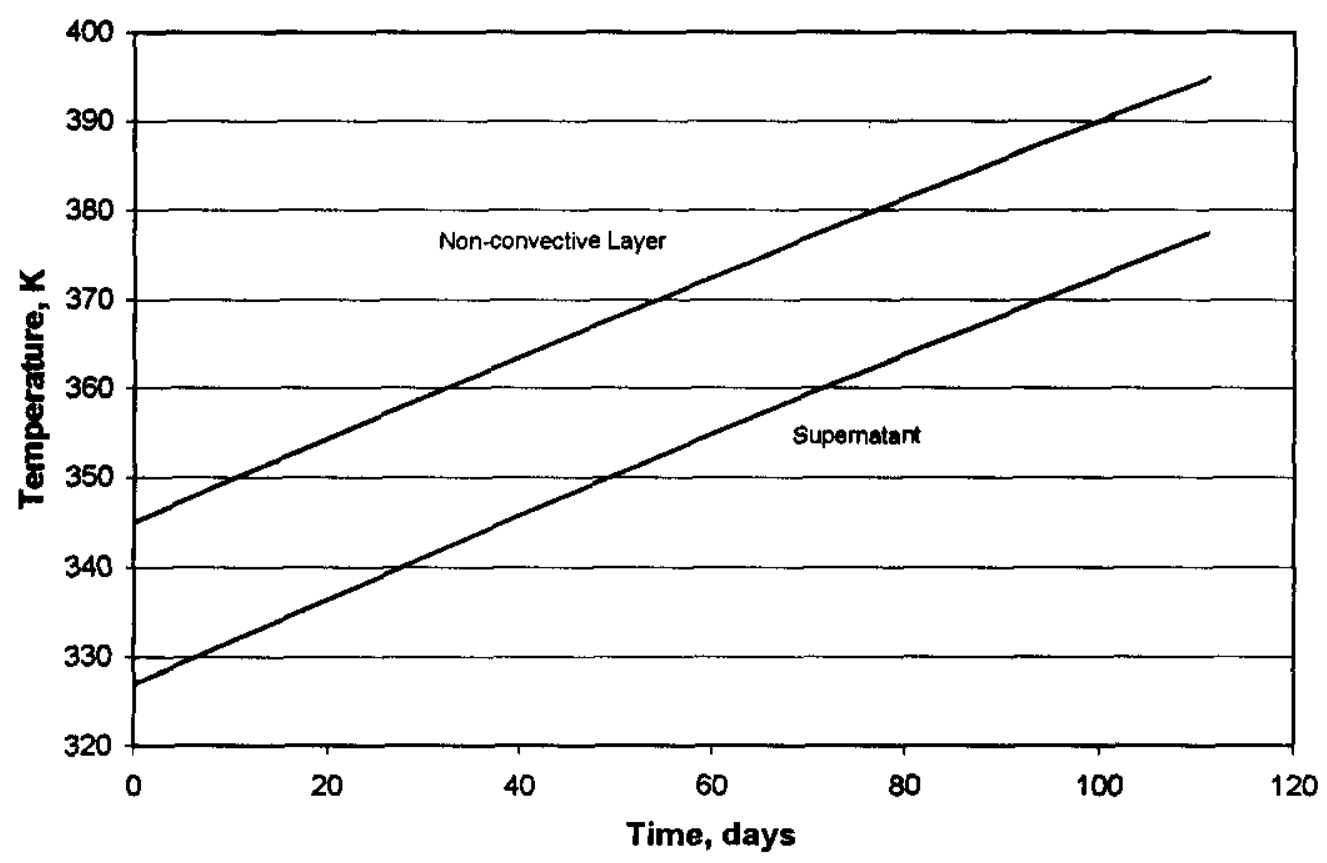


Figure 6-2. 241-AZ-102 Heatup After Loss of Ventilation for Current Conditions.

\section{AZ-102 Heatup After Loss of Ventilation}

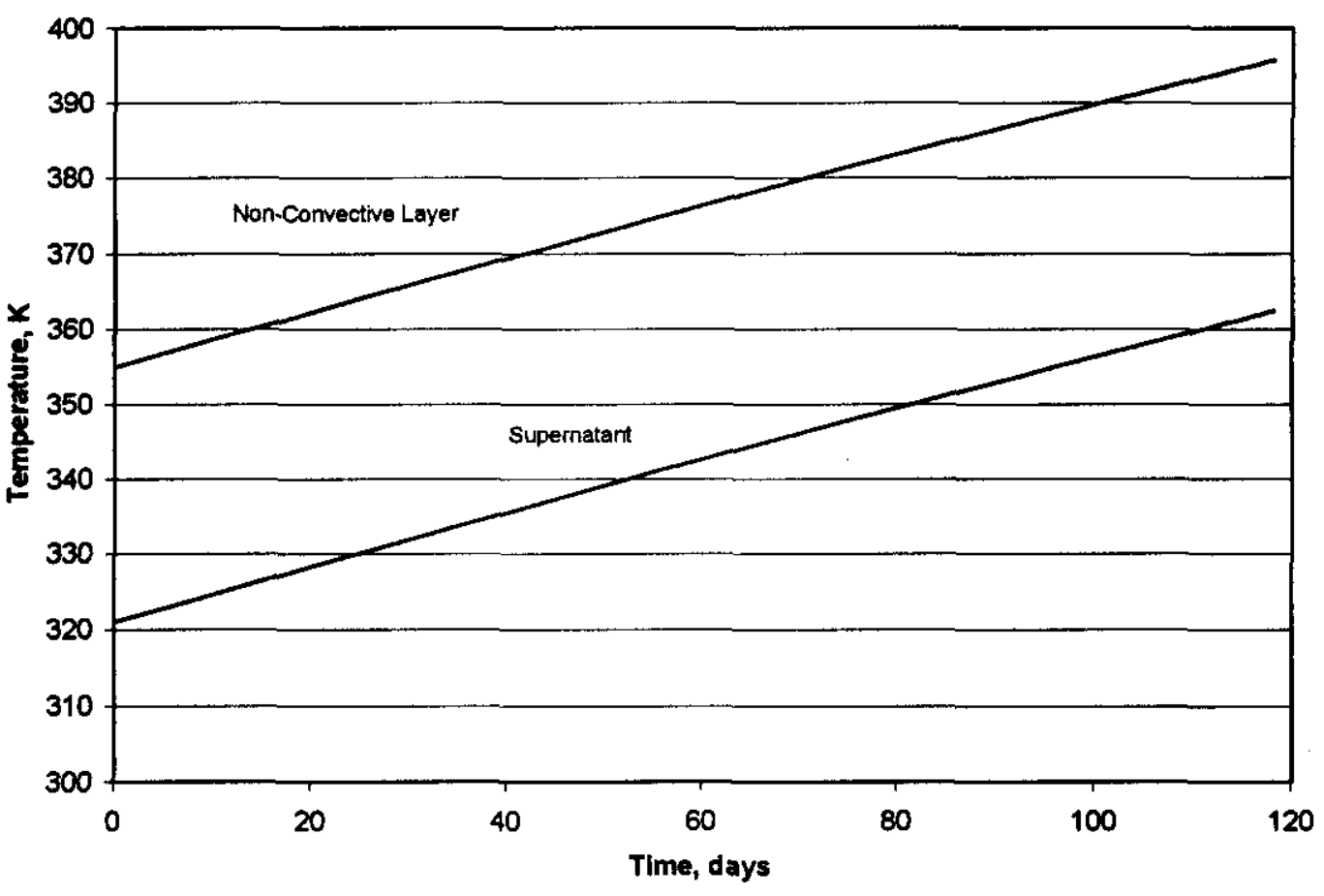


Table 6-4. Time to Bump Conditions for AZ DSTs.

\begin{tabular}{|c|c|c|c|c|c|c|c|c|c|}
\hline DST & $\begin{array}{c}\mathrm{CL}^{(1)} \\
\text { Density, } \\
\left(\mathrm{kg} / \mathrm{m}^{3}\right)\end{array}$ & $\begin{array}{l}\text { NCL } \\
\text { Density, } \\
\text { (kg/m) }\end{array}$ & $\begin{array}{l}T_{\text {N(BCL }} \\
\text { (K)' }\end{array}$ & $\begin{array}{l}T_{C L} \\
(4) \\
\text { (K) }\end{array}$ & $\begin{array}{c}\text { CL } \\
\text { Volume, } \\
\left(\mathrm{m}^{3}\right)\end{array}$ & $\begin{array}{c}\text { NCL } \\
\text { Volume, } \\
\left(\mathrm{m}^{3}\right)\end{array}$ & $\begin{array}{l}\text { NCL Heat } \\
\text { Load, (W) } \\
\text { (Crea et al } \\
\text { 2000) }\end{array}$ & $\begin{array}{c}\text { Tank Heat } \\
\text { Load, (W) } \\
\text { (Barton \& } \\
\text { Bingham, 1999) }\end{array}$ & $\begin{array}{c}\text { Time to } \\
\text { Bump (s), } \\
\text { (days) }\end{array}$ \\
\hline $241-A Z-10$ & 1190 & 1670 & 345 & 327 & 3021 & 178 & 45949 & 77300 & 111 \\
\hline $241-A Z-10$ & 1100 & 1490 & 355 & 321 & 3131 & 394 & 38894 & 63000 & 118 \\
\hline \multicolumn{10}{|c|}{ 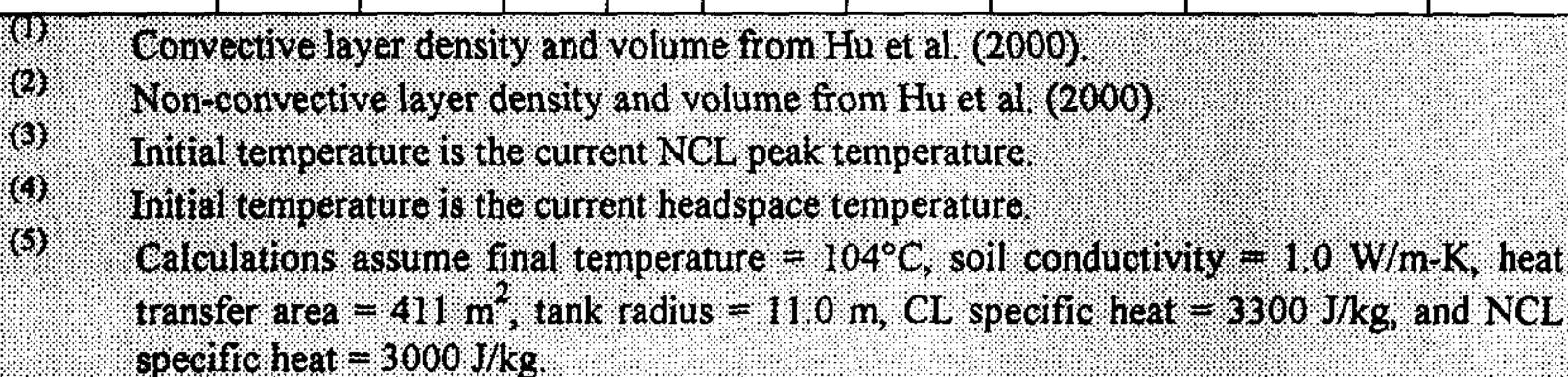 } \\
\hline
\end{tabular}

time to bump value would be about 94 days $(77,300 / 91,200 \cdot 111$ days $)$ and the 241-AZ-102 time to bump value would be about 93 days.

DST 241-AY-102 was not considered here because the time to bump for either 241-AZ-101 or 241-AZ-102 would bound the time to bump for 241-AY-102. 241-AY-102 has a 40,700 W total tank heat load (after the waste transfer from 241-C-106), and initial peak non-convective layer temperature of $350 \mathrm{~K}$. The time to bump for $241-\mathrm{AY}-102$ is therefore roughly $50 \%$ larger than the time to bump for 241-AZ-101 or 241-AZ-102.

Tanks 241-AZ-101, 241-AZ-102, and 241-AY-102 pass screening criteria 1 through 5, and can reach saturation conditions under the loss of cooling scenario. However, none of these tanks can attain neutral buoyancy based on noncondensible gas generation, as shown in Table 4-2, and therefore, none pass screening criterion 6 . Therefore, our best judgement is that no SSTs and DSTs are susceptible to tank bump under safe storage conditions, even for the off-normal loss of cooling scenario.

For one of these tanks, 241-AY-102, this conclusion is weaker than for the others (see Table 4-2, value of criterion). Therefore in Section 7.0, the credibility of such an extended loss-of-cooling scenario is calculated in terms of annual frequency or probability for AZ / AY tank farm. The most important variable in determining the annual frequency of a tank bump is the time to bump.

\subsection{APPLICATION OF TANK BUMP CRITERIA TO WASTE AT LCO CONDITIONS}

The method of Section 6.1 is repeated, except that ventilation controls are not credited. This new assumption is irrelevant to the DSTs listed in Tables 6-1 and 6-2. It is also irrelevant to the calculations in Table 6-3 if the resulting time to saturated conditions value is listed as "Inf." on the far right hand side; i.e., the DST reaches a steady-state temperature below saturation and the choice of initial conditions does not matter. If the time to reach saturated conditions is not infinite, removing ventilation system controls will substantially reduce the time to saturated conditions. 
In this section, Tables 6-3 and 6-4 are generated anew, assuming that the initial conditions are the LCO rather than the current, ventilated DST conditions. LCO conditions are stated in the DST and AWF Tank Waste Temperature Controls LCS/LCO 3.3.2:

The WASTE temperature shall be either:

a. $\quad \leq 195^{\circ} \mathrm{F}$ in all levels of the WASTE,

OR

b. $\leq 195^{\circ} \mathrm{F}$ in the top $15 \mathrm{ft}$. of the WASTE

AND

$\leq 215^{\circ} \mathrm{F}$ in the WASTE below $15 \mathrm{ft}$.

Therefore, calculations in Table 6-3 are repeated assuming the initial condition is $195^{\circ} \mathrm{F}(364 \mathrm{~K})$. Calculations in Table 6-4 are repeated assuming that the supernatant temperature is $195^{\circ} \mathrm{F}$ $(364 \mathrm{~K})$ and the non-convective layer temperature is $215^{\circ} \mathrm{F}(375 \mathrm{~K})$. Results are presented in Tables 6-5 and 6-6.

Table 6-5. Time to Saturated Conditions for DSTs Under LCO.

\begin{tabular}{|c|c|c|c|c|c|c|c|c|c|}
\hline $\begin{array}{l}\text { DST, } \\
\text { 241- }\end{array}$ & $\begin{array}{c}\mathrm{CL}^{(\mathbf{1})} \\
\text { Density, } \\
\left(\mathrm{kg} / \mathrm{m}^{3}\right)\end{array}$ & $\begin{array}{l}\text { NCL }{ }^{(2)} \\
\text { Density, } \\
\left(\mathrm{kg} / \mathrm{m}^{3}\right)\end{array}$ & $\stackrel{\mathbf{T}_{0}{ }^{(3)},}{(\mathrm{B})}$ & $\begin{array}{c}\text { CL } \\
\text { Volume, } \\
\left(\mathrm{m}^{3}\right)\end{array}$ & $\begin{array}{c}\text { NCL } \\
\text { Volume, } \\
\left(\mathrm{m}^{3}\right)\end{array}$ & $\begin{array}{l}\text { Barton \& } \\
\text { Bingham } \\
\text { Tank Heat } \\
\text { Load, (W) }\end{array}$ & $\begin{array}{l}\text { Hu et al. } \\
\text { Tank Heat } \\
\text { Load, (W) }\end{array}$ & $\begin{array}{c}\text { Barton \& } \\
\text { Bingham } \\
\text { Time }{ }^{(4)} \text {, } \\
\text { (days) }\end{array}$ & $\begin{array}{c}\text { Hu et al. } \\
\text { Time }{ }^{(4)} \text {, } \\
\text { (days) }\end{array}$ \\
\hline SY-101 & 1390 & 1610 & 364 & 2286 & 2214 & 16100 & 10600 & 739 & Inf. \\
\hline AN-103 & 1490 & 1710 & 364 & 2074 & 1552 & 18700 & 12100 & 390 & Inf. \\
\hline AN-104 & 1400 & 1590 & 364 & 2286 & 1700 & 19700 & 13700 & 350 & 1503 \\
\hline AN-105 & 1420 & 1580 & 364 & 2411 & 1851 & 13400 & 9340 & 1968 & Inf. \\
\hline$A W-101$ & 1400 & 1630 & 364 & 3104 & 1158 & 15200 & 10300 & 848 & Inf. \\
\hline $\mathrm{AN}-102$ & 1410 & 1500 & 364 & 3676 & 337 & 11500 & 9340 & Inf. & Inf. \\
\hline AN-107 & 1370 & 1560 & 364 & 3017 & 935 & 13700 & 11700 & 1397 & Inf. \\
\hline (2) & $\mathrm{ln}^{2} \mathrm{r}$ & acher & 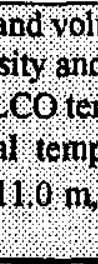 & 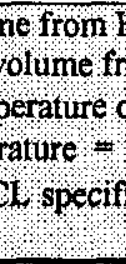 & $95 \%$ & $=00$, & \% & & $\mathrm{fer}$ area $\mathrm{lnf}$ \\
\hline
\end{tabular}


Table 6-6. Time to Saturated Conditions for AZ DSTs Under LCO Conditions.

\begin{tabular}{|c|c|c|c|c|c|c|c|c|c|}
\hline DST & $\begin{array}{c}\text { CL }^{(1)} \\
\text { Density, } \\
\text { (kg/m) }\end{array}$ & $\begin{array}{l}\text { NCL (2) } \\
\text { Density, } \\
\left(\mathrm{kg} / \mathrm{m}^{3}\right)\end{array}$ & $\begin{array}{l}\mathbf{T}_{\mathrm{NCL}} \\
\text { (K) } \\
\text { (K) }\end{array}$ & $\begin{array}{l}T_{\text {CI. }} \\
(4) \\
(K)\end{array}$ & $\begin{array}{c}\text { CL } \\
\text { Volume, } \\
\left(\mathrm{m}^{3}\right)\end{array}$ & $\begin{array}{c}\text { NCL } \\
\text { Volume, } \\
\left(\mathrm{m}^{3}\right)\end{array}$ & $\begin{array}{c}\text { NCL Heat } \\
\text { Load, (W) } \\
\text { (Crea et al } \\
2000) \\
\end{array}$ & $\begin{array}{c}\text { Tank Heat } \\
\text { Load, (W) } \\
\text { (Barton \& } \\
\text { Bingham, } \\
\text { 1999) } \\
\end{array}$ & $\begin{array}{l}\text { Time to } \\
\text { Saturated } \\
\text { Conditions } \\
\text { (s), (days) }\end{array}$ \\
\hline 241-AZ-10 & 1190 & 1670 & 375 & 364 & 3021 & 178 & 45949 & 77300 & 30 \\
\hline 241-AZ-10 & 1100 & 1490 & 375 & 364 & 3131 & 394 & 38894 & 63000 & 38 \\
\hline \multicolumn{10}{|c|}{ 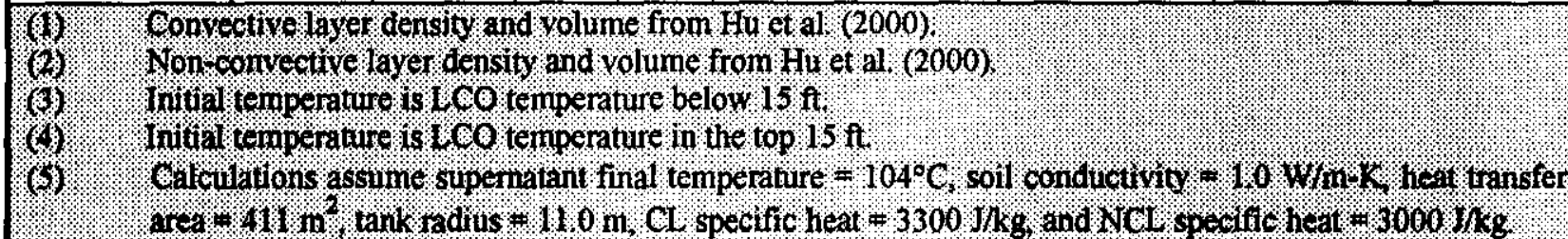 } \\
\hline
\end{tabular}

Table 6-5 can be compared against Table 6-3. Using the LCO assumption reduces the time to saturated conditions by $50 \%$ to $70 \%$ relative to the values in Table $6-3$, because the temperature rise from initial to final conditions is much smaller. For instance, in the calculation summarized by Table 6-3, the 241-SY-101 waste temperature must rise from $323 \mathrm{~K}$ to $377 \mathrm{~K}$. In the calculation summarized by Table 6-5, the 241-SY-101 waste temperature must rise from $364 \mathrm{~K}$ to $377 \mathrm{~K}$, or about $25 \%$ of the temperature rise assumed for first calculation (Table 6-3). The time to saturated conditions then falls from 2028 days to 739 days, which is a $64 \%$ drop in the number of required days. The percentage drop in the number of days (64\%) corresponds roughly to percentage drop in the number of degrees from the initial temperature to saturated conditions $(75 \%)$. Agreement would be perfect given negligible upward and downward conduction losses, but this is not the case for 241-SY-101.

For the DSTs listed in Table 6-5, applying the bump criteria under the LCO assumption does not change conclusions. The times listed in Table 6-5 still exceed any conceivable repair times and tank bumps in these DSTs can be neglected as a practical matter.

Time to bump conditions was re-calculated for the AZ DSTs, assuming LCO conditions. Results are listed in Table 6-6, which can be compared to Table 6-4. Temperature histories are shown in Figures 6-3 and 6-4.

Time to bump condition values listed in Table 6-6 are about 70\% smaller than the values listed in Table 6-4, which is expected because the required supernatant temperature rise has been reduced by about $70 \%$ (from roughly $50^{\circ} \mathrm{C}$ to $14^{\circ} \mathrm{C}$ ). At these high tank heat loads, the time to bump conditions is about proportional to the difference between final and initial temperatures, and conduction heat losses can be neglected to a good approximation. Also, the time to tank bump is proportional to the inverse of tank heat load. Time to bump for 241-AY-102 would be about 50 days, or 50\% larger than the values shown in Table 6-6. Using the Hu et al. (2000) values, gives 26 days for $241-\mathrm{AZ}-101$ and 29 days for $241-\mathrm{AZ}-102$. 


\section{RPP-6213 REV 0}

Figure 6-3. 241-AZ-101 Heatup After Loss of Ventilation - LCO Conditions.

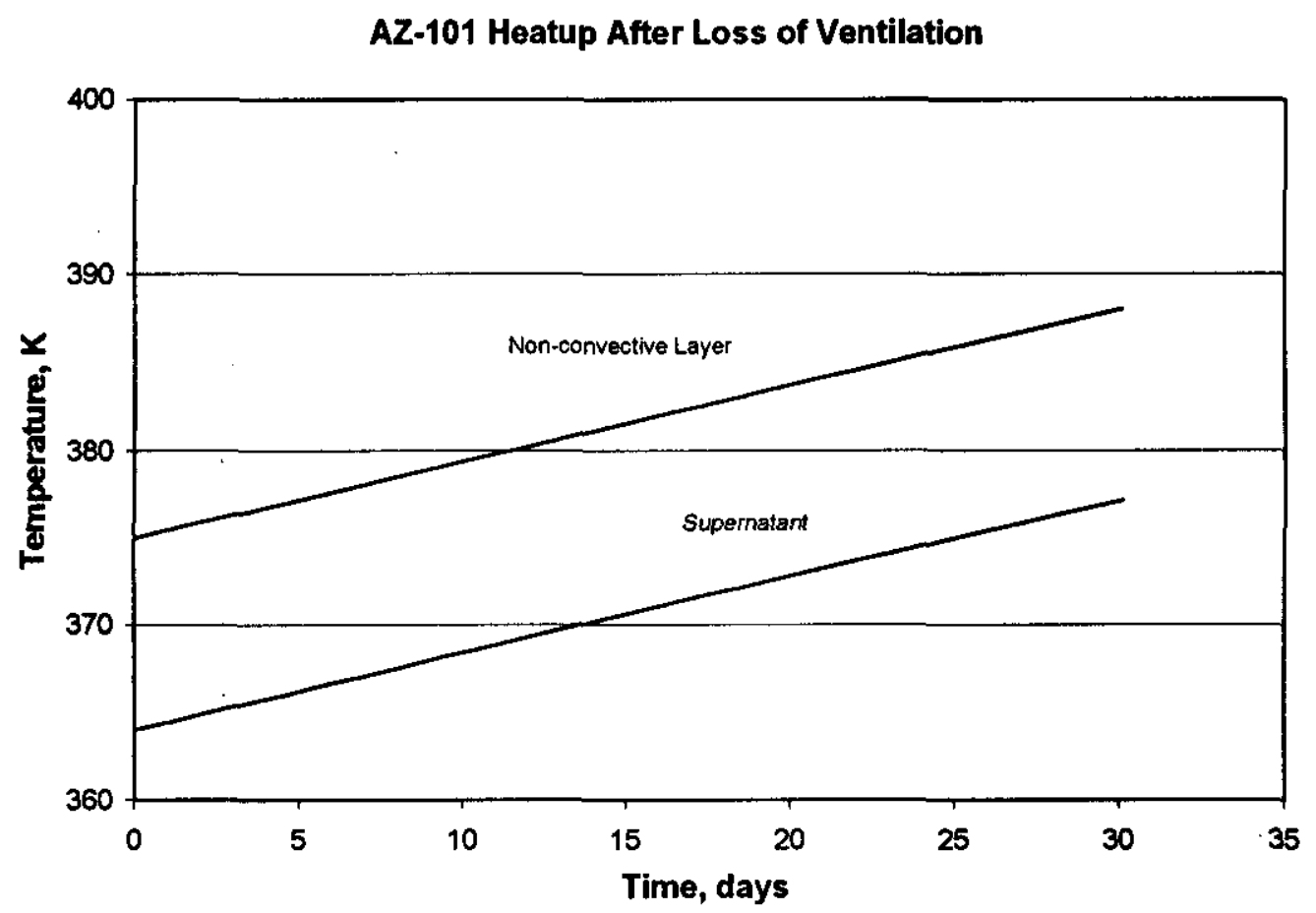




\section{RPP-6213 REV 0}

Figure 6-4. 241-AZ-102 Heatup After Loss of Ventilation - LCO Conditions.

\section{AZ-102 Heatup After Loss of Ventilation}

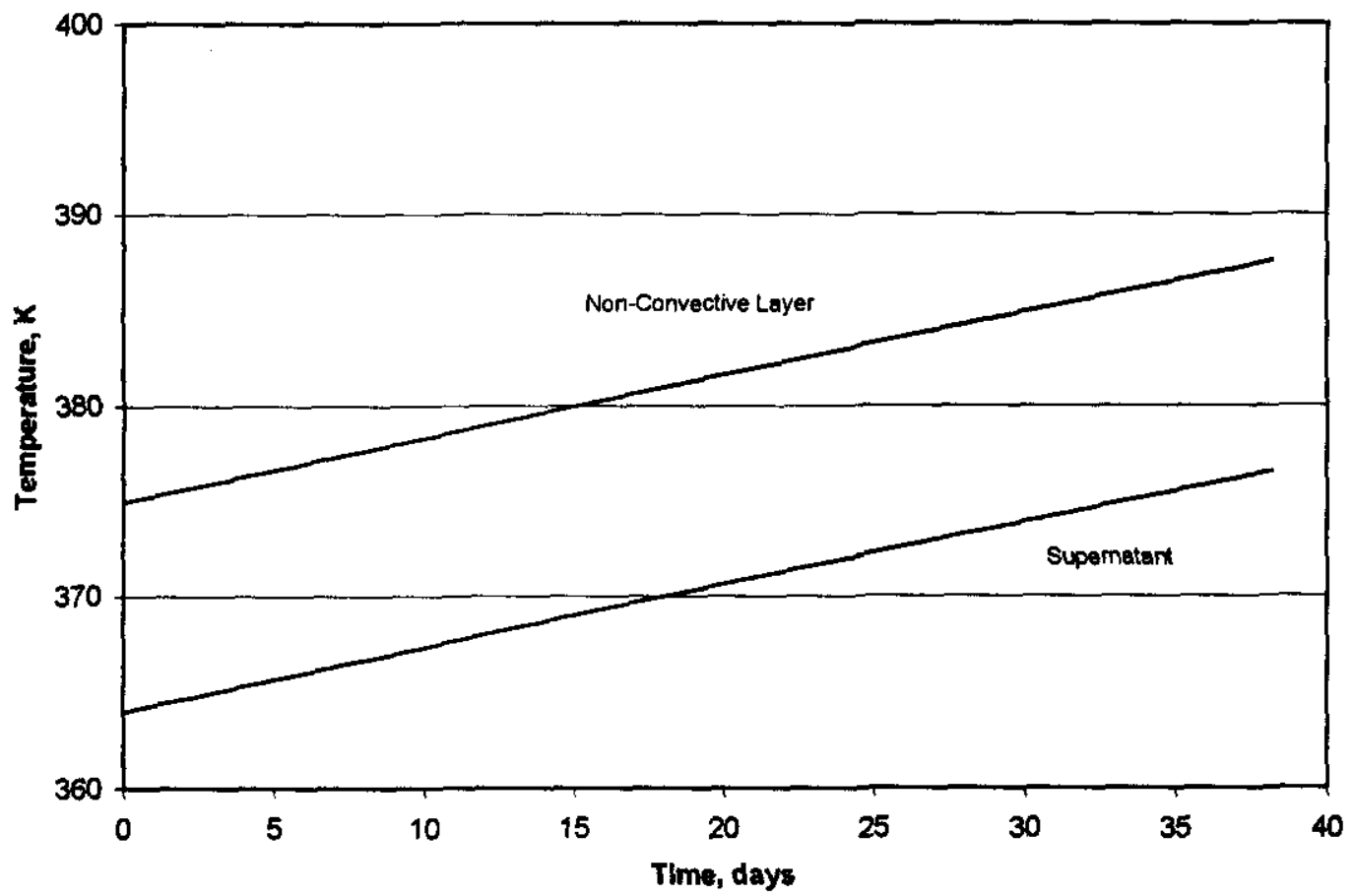




\subsection{APPLICATION TO HISTORIC EVENTS}

The historic events shown in Table 3-1 can be better understood in light of the discussion in this section. The most important aspect of Table 3-1 is that the tank heat rates greatly exceed even the highest current tank heat loads. For the historic tanks, the time to bump conditions would have been much less than the 100 days or so predicted for the present AZ DSTs, and without a sufficient ventilation flowrate, bumping could have been expected shortly after the tank was filled, even without an initiator. For example, tank 241-S-104 was filled in July 1953, and began bumping in October of 1953, despite the installation of auxiliary water-cooled condensers (Tomlinson 1955). In tank 241-S-101, bumping began in January 1954. In both cases, the waste temperature at the bottom probably exceeded the saturation temperature at the bottom hydrostatic pressure. This was definitely the case in the other events, where the temperature at the bottom was anywhere between $260^{\circ} \mathrm{F}$ and $357^{\circ} \mathrm{F}$. This can be compared to the value used in this section as the bump criterion for, say, tank $241-\mathrm{AZ}-101$, which is $398 \mathrm{~K}$, or $257^{\circ} \mathrm{F}$.

Finally, we note that a number of later bump events were initiated by ALC restart after shutdown, and observed behavior is in qualitative according to expectations based on Section 4.1 .

\subsection{NOTE ON SCREENING TANK BUMP POTENTIAL}

The criteria described and applied above may be used continually as reference waste tank conditions change, for example, if tank power or layer inventory estimates change due to new characterization data or as a result of waste transfers. However, operations which change the waste temperature can change the calculated time to attain saturation conditions used by criteria 4 and 5. New times to saturation must be calculated and correctly compared with time to restore tank ventilation. The methodology for consideration of repair or restoration is discussed in Section 7. 
RPP-6213 REV 0

This page intentionally left blank. 


\subsection{SCENARIO QUANTIFICATION}

A tank bump requires loss of active waste cooling functions for an extended period, given the requisite conditions of waste volumetric heat generation, supernatant depth, etc., described in the previous section. An extended loss of cooling has four major initiating events: (1) random component failures or common cause failures, (2) loss of off-site power events (LOSP), (3) seismic events, and (4) lightning strikes. The purpose of this section is to estimate the frequency of tank bump conditions due to each of these contributors, accounting for potential recovery within the available interval. For the random component and common cause failures, the existing reliability, availability, and maintainability (RAM) studies for waste feed delivery will be used for failure frequencies and repair times. The goal here is not necessarily to provide a RAM of safe storage operations, but rather to estimate whether tank bumps are anticipated $\left(10^{-2} / \mathrm{yr}\right.$ to $\left.10^{0} / \mathrm{yr}\right)$, unlikely $\left(10^{-4} / \mathrm{yr}\right.$ to $\left.10^{-2} / \mathrm{yr}\right)$, or extremely unlikely $\left(10^{-6} / \mathrm{yr}\right.$ to $\left.10^{-4} / \mathrm{yr}\right)$.

The annual frequency of a tank bump event is expressed as a function of the ventilation system annual failure frequency and the conditional probability that the ventilation system cannot be recovered (restored or repaired) within the available time interval. A general expression for the annual tank bump frequency, $F$, is given by:

$$
F=\sum_{i=1}^{4} F_{i}=\sum_{i=1}^{4} \int_{0}^{\infty} p_{i}\left(\lambda_{i}\right) P_{n r i}\left(t_{a}, \lambda_{i}\right) d \lambda_{i}
$$

where $i$ is an index for one of the four initiators listed above, $p(\lambda)$ is the annual failure or hazard probability distribution as a function of some parameter $\lambda$, and $P_{n r}\left(t_{a}, \lambda\right)$ is the conditional probability that ventilation cannot be recovered as a function of the available time $t_{a}$ and $\lambda$. Recovery from random failures or lightning strikes is independent of the initiator, which is not the case for a LOSP or seismic event. For LOSP, the conditioning parameter is the duration of the event; and for seismic events, the conditioning parameter is the magnitude of the event, which is typically expressed in terms of peak horizontal acceleration. For random failure and lightning strikes, the failure to recover probability is independent of conditions related to the initiating event, in which case,

$$
F_{i}=f_{i} P_{n r i}\left(t_{a}\right)
$$

Each of the initiating events listed above is evaluated in the balance of this section.

\subsection{VENTILATION SYSTEM FAILURE AND FAILURE TO RECOVER}

The frequency analysis considers the LCO assumption first and then the current waste conditions. The LCO assumption must be considered bounding because it results in a much shorter time to bump conditions, which is key to the time available for recovery. Recovery times are considered first and then used to evaluate mean ventilation system failure rate. 
In a study that presents the probability of schedule delays for delivery of $\mathrm{HLW}$ feed batches to the customer, the reliabilities of the primary HVAC, annular HVAC, HVAC ancillary systems, air lift circulators, and miscellaneous support systems have been considered for 241-AZ-101 waste transfer operations (Carlson 1999 and 1999a). Schedule delays were estimated by creating individual RAM models for each of the following contributors: primary HVAC, annulus HVAC, service air, transfer system, monitoring system, recirculation condenser, human errors, ventilation condenser system, and external events. In the RAM, the ALC system is modeled within the service air system. The primary HVAC system consists of individual air inlets, filters, an exhaust condenser, a high efficiency mist eliminator, redundant exhaust fans and filter banks, and an exhaust stack with radiological sampling equipment. Individual RAM models were built for the annular HVAC, ventilation condenser cooling, and recirculation condenser cooling.

By using 10,000 simulated waste transfer operations, Carlson's (1999a) studies found the number of "off-normal" events that occurred and the delay due to each event. In summary, out of 10,000 simulated batch transfer events, 8 delay events can be expected on average, with a mean delay per event of 43 hours. The breakdown by system contribution is shown in Table 7-1. The results of this study will be adapted to find recovery times for loss of ventilation under safe storage conditions. A key assumption is that the individual recovery times for restoration and/or corrective maintenance as determined by the WFD RAM can be applied to the safe storage scenario.

Table 7-1. Critical Items List for the High Level Waste Transfer RAM B, Ranked by the Contribution of System Type to Expected Schedule (Carlson 1999a).

\begin{tabular}{|l|c|c|c|c|c|}
\hline \multicolumn{1}{|c|}{ System Type } & $\begin{array}{c}\text { Expected } \\
\text { No. of } \\
\text { Delay } \\
\text { Events }\end{array}$ & $\begin{array}{c}\text { Expected } \\
\text { Delay per } \\
\text { Event } \\
\text { (hrs) }\end{array}$ & $\begin{array}{c}\text { Total } \\
\text { Expected } \\
\text { Delay } \\
\text { Time (hrs) }\end{array}$ & $\begin{array}{c}\text { Percent } \\
\text { Contribution } \\
\text { to No. of } \\
\text { Events }\end{array}$ & $\begin{array}{c}\text { Percent } \\
\text { Contribution } \\
\text { to Total } \\
\text { Delay }\end{array}$ \\
\hline Total & 8.0 & 43 & 341 & $100 \%$ & $100 \%$ \\
\hline HVAC Primary & 2.32 & 37 & 85 & $29.0 \%$ & $25.0 \%$ \\
\hline HVAC Annulus & 1.22 & 52 & 64 & $15.3 \%$ & $18.7 \%$ \\
\hline Transfer & 2.23 & 28 & 62 & $28.0 \%$ & $18.2 \%$ \\
\hline Service Air & 0.61 & 88 & 54 & $7.7 \%$ & $15.9 \%$ \\
\hline Monitoring & 0.22 & 167 & 37 & $2.8 \%$ & $10.9 \%$ \\
\hline Vent Cond. Cooling System & 0.38 & 27 & 10 & $4.8 \%$ & $3.0 \%$ \\
\hline Raw Water & 0.10 & 31 & 3 & $1.2 \%$ & $0.9 \%$ \\
\hline Dilution/Flush & 0.01 & 168 & 2 & $0.1 \%$ & $0.6 \%$ \\
\hline Nitrogen & 0.00 & 1006 & 2 & $0.02 \%$ & $0.6 \%$ \\
\hline Instrument Air & 0.06 & 30 & 2 & $0.7 \%$ & $0.5 \%$ \\
\hline Process Air & 0.04 & 29 & 1 & $0.5 \%$ & $0.4 \%$ \\
\hline Service Water & 0.003 & 131 & 0.4 & $0.04 \%$ & $0.1 \%$ \\
\hline Instrumentation & 0.01 & 34 & 0.3 & $0.1 \%$ & $0.1 \%$ \\
\hline Electric Power & 0.01 & 6 & 0.03 & $0.1 \%$ & $0.01 \%$ \\
\hline
\end{tabular}




\section{RPP-6213 REV 0}

\subsubsection{Failure to Recover Probability}

Failure to recover probability consists of two components: failure to restore ventilation within the available time and failure to enact corrective maintenance within the available time. Restoration considers that ventilation system functions can be recovered without repair in many instances by manual switchover for functions with redundant hardware. This was recognized and accounted for in the waste transfer RAM by developing probability density functions for restoration times and determining whether recovery from an off-normal event required a restoration function or a corrective maintenance function. A key assumption was that if the first component failed, the backup component would run with negligible failure rate until the main component could be repaired. For the most part, restoration functions inside a tank farm fence required an expected value (mode) of one day, with a maximum time of one week.

From Table 7-1, it is clear that most, but not all, recoveries involved restoration actions rather than corrective maintenance actions. This is because expected delays were on the order of two days, which is much less than the expected corrective maintenance times. A description of corrective maintenance activities is given in Table 7-2, and $\log$-normal distributions (low $=5^{\text {th }}$ percentile and high $=95^{\text {th }}$ percentile) for clock hours to complete these activities are shown in Table 7-3. From Table 7-2, activities requiring tank farm radiological access are considered to estimate corrective maintenance times; in response to ventilation system failures, activities required pit or tanks access are not expected, as are activities not requiring radiological access. Activities requiring tank farm radiological access have identifiers TRISA, TRIEL, TCIEL, TCNSA, and TCNEL. For these activities, $200 \mathrm{hrs}$. is representative of a mean time for corrective maintenance. To estimate the fraction of recovery activities that are restoration functions rather than corrective maintenance activities, use the following expression:

$$
t_{d}=x_{r s} t_{r s}+\left(1-x_{r s}\right) t_{C M}
$$

where $t_{d}$ is the mean delay time, $x_{r s}$ is the fraction of events that requires restoration rather than corrective maintenance, $t_{\mathrm{rs}}$ is the mean restoration time, and $\mathrm{t}_{\mathrm{CM}}$ is the mean corrective maintenance time. Assuming the mean delay time is 37 hours, the mean restoration time is 24 hours and the mean corrective maintenance time is 200 hours, $x_{r s}$ equals $93 \%$. Assuming the mean restoration time is very small, but the mean corrective maintenance time is still $200 \mathrm{hrs}$, $\mathrm{x}_{\mathrm{rs}}$ equals $82 \%$. In application, $\mathrm{x}_{\mathrm{rs}}$, will be treated as a uniform random variable on the interval from 80 to $90 \%$.

The probability that ventilation is not recovered in the available time is then:

$$
P_{n r R}=\left(1-x_{r s}\right) \sum_{n=1}^{N} x_{n}\left[1-\log -\operatorname{normal}\left(t_{a}, \alpha_{n}, \beta_{n}\right)\right]
$$

where $\mathrm{N}$ is the number of corrective maintenance activities, $\mathrm{x}$ is the fraction of all $\mathrm{CM}$ activities for the $n^{\text {th }} \mathrm{CM}$ activity (the relative frequency such that the sum of all $x_{n}$ values is equal to one), $\log$-normal is the cumulative log-normal distribution function evaluated at the available time, $t_{a}$, for the $\log$-mean $\alpha_{n}$ and $\log$-standard deviation, $\beta_{n}$, of the $n^{\text {th }} C M$ activity. This expression 
Table 7-2. Component Types and Recovery Conditions (Carlson 1999).

\begin{tabular}{|c|c|c|c|c|c|c|c|c|c|c|}
\hline \multirow{2}{*}{$\begin{array}{l}\text { Recovery } \\
\text { ID }\end{array}$} & \multirow{2}{*}{ Basic Description } & \multicolumn{9}{|c|}{ Component Repair Effort Category } \\
\hline & & 1 & 2 & 3 & 4 & 5 & 6 & 7 & 8 & 9 \\
\hline PRIML & $\begin{array}{l}\text { Definition: Pit or Tank Access, Remote Handled, Moderate } \\
\text { Planning, Long Lead Spares. Examples: new or a replacement } \\
\text { jumper, initial installation of a mixer pump. }\end{array}$ & & & & & $\mathbf{X}$ & & & & \\
\hline PRIEL & $\begin{array}{l}\text { Definition: Pit or Tank Access, Remote Handled, Extensive } \\
\text { Planning. Long Lead Spares. Examples: Pump removal } \\
\text { /replacement, thermocouple tree removal/replacement. }\end{array}$ & $\mathbf{X}$ & & & & $\mathbf{X}$ & & & $\mathbf{X}$ & \\
\hline PCIML & $\begin{array}{l}\text { Definition: Pit or Tank Access, Contact Handled, Moderate } \\
\text { Planning, Long Lead Spares. Example: Replace valve/piping } \\
\text { components (waste contacted surfaces) on an existing jumper } \\
\text { that has been removed and is intended to be reinstalled. }\end{array}$ & & & & & $\mathbf{X}$ & & & $\mathbf{X}$ & \\
\hline PCIEL & $\begin{array}{l}\text { Definition: Pit Access Only (no tank access), Contact } \\
\text { Handled, Extensive Plasning. Long Lead Spares. Examples. } \\
\text { This includes, for example, the investigation and repair of } \\
\text { frozen or non-rotating pump impellers, the replacement of } \\
\text { valve actuator components. }\end{array}$ & $X$ & & $\mathbf{X}$ & & $\mathbf{X}$ & & & $\mathbf{X}$ & \\
\hline TRISA & $\begin{array}{l}\text { Definition: Tank Farm Rad area access, Remote Handied } \\
\text { /ntrusive, Simple Planning, Spares Available. Examples: pre- } \\
\text { approved maintenance procedure, semi-routine actions. }\end{array}$ & & & & & & & & & \\
\hline TRIEL & $\begin{array}{l}\text { Definition: Tank Farm Rad area access, Remote Handled, } \\
\text { Moderator to Extensive Planning, Long Lead Spares. } \\
\text { Examples: Removal/replacement of vent system de-entrainer } \\
\text { or mist eliminator elements. }\end{array}$ & & & & & $\mathbf{X}$ & & & & \\
\hline TCISA & $\begin{array}{l}\text { Definition: Tank Farm Rad area access, Contact Handled } \\
\text { Intrusive, Simple Planning, Spares Available. Examples: } \\
\text { Routine removal/replacement of consumable components, } \\
\text { process instrument (pressure, temperature, and flow device) } \\
\text { calibrations. }\end{array}$ & $\mathbf{X}$ & & & & $X$ & $\mathbf{X}$ & & & \\
\hline TCIEL & $\begin{array}{l}\text { Definition: Tank Farm Rad area, Contact Handled/Intrusive, } \\
\text { Mod to Extensive Planning, Long Lead Spares. Examples: } \\
\text { Remove/replace ENRAF, contaminated piping/ducting weld } \\
\text { repairs, primary vent heater element replacement. }\end{array}$ & & & $X$ & & $X$ & & & & \\
\hline TCNSA & $\begin{array}{l}\text { Definition: Tank Farm Rad area access, Non-intrusive (non- } \\
\text { Contact), Simple Planning, Spares Available. Examples: } \\
\text { replacing roughing (pre) filters, CAM vacuum pump } \\
\text { replacement. }\end{array}$ & $X$ & $X$ & $X$ & & $X$ & $X$ & $\mathbf{X}$ & & $\mathbf{X}$ \\
\hline TCNEL & $\begin{array}{l}\text { Definition: Tank Farm Rad area access, Non-intrusive, Mod to } \\
\text { Extensive Planning, Long Lead Spares. Examples: Non- } \\
\text { routine maintenance on electrical feeds/motor control centers, } \\
\text { primary vent system fan wheel replacement. }\end{array}$ & $X$ & $X$ & $\mathbf{X}$ & & $X$ & & & & \\
\hline NCNNA & $\begin{array}{l}\text { Definition: Non-Radiological area access, Minimum Planning, } \\
\text { Spares Available. Examples: Infrastructurc repairs, site utility, } \\
\text { and Refrigeration and Equipment Services (RES) team support. }\end{array}$ & $X$ & $\mathbf{X}$ & $\mathbf{X}$ & $\mathbf{X}$ & $\mathbf{X}$ & $\mathbf{X}$ & & & $\mathbf{X}$ \\
\hline NCNEL & $\begin{array}{l}\text { Definition: Non-Radiological area access, Moderate to } \\
\text { Extensive Planning, Long Lead Spares. Examples: major } \\
\text { component rebuilds/replacements. }\end{array}$ & $X$ & & $\mathbf{X}$ & $\mathbf{X}$ & $\mathbf{X}$ & & $\mathbf{X}$ & & \\
\hline \multicolumn{11}{|c|}{ Component Repair Effort Categories: $\quad 5=$ Mechanical General } \\
\hline \multicolumn{4}{|c|}{$1=I \& C$ General } & \multicolumn{7}{|c|}{$6=$ HVAC Special } \\
\hline \multicolumn{4}{|c|}{$2=I \& C$ Special } & & $=$ & Com & pute & & & \\
\hline \multirow{2}{*}{\multicolumn{4}{|c|}{$\begin{array}{l}3=\text { Electrical On-Site } \\
4=\text { Electrical Off-Site }\end{array}$}} & \multicolumn{7}{|c|}{$8=$ Mechanical Major } \\
\hline & \multicolumn{3}{|c|}{4 = Electrical Off-Site } & \multicolumn{7}{|c|}{9} \\
\hline
\end{tabular}


Table 7-3. Summary of Corrective Maintenance (CM) Requirements (Clock Hours and Effort) (Carlson 1999).

\begin{tabular}{|c|c|c|c|c|c|}
\hline \multirow{2}{*}{ Recovery Time Description } & \multirow{2}{*}{$\begin{array}{l}\text { Recovery } \\
\text { Time ID }\end{array}$} & \multirow{2}{*}{$\begin{array}{l}\text { Range } \\
\text { Factor }\end{array}$} & \multicolumn{3}{|c|}{$\begin{array}{l}\text { Clock Hours Required to Complete } \\
\text { a CM }\end{array}$} \\
\hline & & & Low & Mean & High \\
\hline I\&C COMP, NCNEL & 1NCNEL & 2 & 44 & 96 & 176 \\
\hline I\&C COMP, NCNNA & 1NCNNA & 1.5 & 23 & 36 & 52 \\
\hline I\&C COMP, PCIEL & 1PCIEL & 3.5 & 185 & 865 & 2,266 \\
\hline I\&C COMP, PRIEL & 1PRIEL & 3 & 158 & 592 & 1,422 \\
\hline I\&C COMP, TCISA & 1TCISA & 2 & 46 & 101 & 184 \\
\hline I\&C COMP, TCNEL & 1TCNEL & 2.5 & 54 & 158 & 338 \\
\hline I\&C COMP, TCNSA & 1TCNSA & 2 & 31 & 68 & 124 \\
\hline I\&C SPECIAL, NCNNA & 2NCNNA & 1.5 & 26 & 40 & 59 \\
\hline I\&C SPECIAL, TCNEL & 2TCNEL & 2.5 & 56 & 163 & 350 \\
\hline I\&C SPECIAL, TCNSA & 2TCNSA & 2 & 34 & 74 & 136 \\
\hline ELEC ONSITE, NCNEL & 3NCNEL & 2 & 52 & 114 & 208 \\
\hline ELEC ONSITE, NCNNA & 3NCNNA & 1.5 & 25 & 39 & 56 \\
\hline ELEC ONSITE, PCIEL & 3PCIEL & 3.5 & 155 & 725 & 1,899 \\
\hline ELEC ONSITE, TCIEL & $3 \mathrm{TCIEL}$ & 2.5 & 123 & 359 & 769 \\
\hline ELEC ONSITE, TCNEL & 3TCNEL & 2.5 & 64 & 187 & 400 \\
\hline ELEC ONSITE, TCNSA & 3TCNSA & 2 & 30 & 66 & 120 \\
\hline ELEC OFFSITE, NCNEL & 4NCNEL & 2 & 8 & 17 & 32 \\
\hline ELEC OFFSITE, NCNNA & 4NCNNA & 1.5 & 6 & 9 & 14 \\
\hline MECH COMP, NCNEL & 5NCNEL & 2 & 41 & 90 & 164 \\
\hline MECH COMP, NCNNA & 5NCNNA & 1.5 & 26 & 40 & 59 \\
\hline MECH COMP, PCIEL & SPCIEL & 3.5 & 223 & 1,043 & 2,732 \\
\hline MECH COMP, PCIML & 5PCIML & 3.5 & 212 & 992 & 2,597 \\
\hline MECH COMP, PRIEL & SPRIEL & 3 & 207 & 776 & 1,863 \\
\hline MECH COMP, PRIML & SPRIML & 3 & 186 & 697 & 1,674 \\
\hline MECH COMP, TCIEL & 5TCIEL & 2.5 & 102 & 298 & 638 \\
\hline MECH COMP, TCISA & 5TCISA & 2 & 71 & 155 & 284 \\
\hline MECH COMP, TCNEL & 5TCNEL & 2.5 & 53 & 155 & 331 \\
\hline MECH COMP, TCNSA & 5TCNSA & 2 & 30 & 66 & 120 \\
\hline MECH COMP, TRIEL & 5TRIEL & 2 & 74 & 162 & 296 \\
\hline HVAC SPECIAL, NCNNA & 6NCNNA & 1.5 & 28 & 43 & 63 \\
\hline HVAC SPECIAL, TCISA & 6TCISA & 2 & 45 & 98 & 180 \\
\hline HVAC SPECIAL, TCNSA & 6TCNSA & 2 & 35 & 76 & 140 \\
\hline COMPUTER, NCNEL & 7NCNEL & 2 & 47 & 103 & 188 \\
\hline COMPUTER, TNTSA & 7TCNSA & 2 & 38 & 83 & 152 \\
\hline MECH MAJOR, PCIEL & 8PCIEL & 3.5 & 660 & 3,087 & 8,085 \\
\hline MECH MAJOR, PCIML & 8PCIML & 3.5 & 441 & 2,063 & 5,402 \\
\hline MECH MAJOR, PRIEL & 8PRIEL & 3 & 532 & 1,995 & 4,788 \\
\hline SUP FACILITY, NCNNA & 9NCNNA & 1.5 & 16 & 25 & 36 \\
\hline SUP FACILITY, TCNSA & 9TCNSA & 2 & 33 & 72 & 132 \\
\hline
\end{tabular}


assumes that restoration activities succeed during the available time, which is justifiable because the restoration time is about one day while the available time is about one month. This expression states that the probability that CM fails is the sum of the probabilities that individual $\mathrm{CM}$ activities fail. The probability that an individual $\mathrm{CM}$ activity will fail in the available time is just the exceedance probability for the log-normal distribution of repair time. Exceedance probability for each individual $\mathrm{CM}$ activity is weighted by the relative frequency of each $\mathrm{CM}$ activity.

On the basis of Table 6-5 and the discussion in Section 6.2, the time to heatup to bump conditions, $t_{b}$ is assumed to be anywhere between 25 and 35 days. The available time $t_{a}$ is taken to be the time to heatup to saturated conditions minus the time needed to recognize the loss of ventilation and initiate corrective maintenance, $t_{s}$. For the $\mathrm{LCO}$ assumption, waste temperature surveillance occurs at a ten-day frequency and the time $t_{s}$ is assumed to be uniform on the interval between 1 and 10 days. Based on Table H-6 of the Carlson (1999) reference, only the 3TCNEL, 5TCISA, and 5TCIEL CM activities are relevant and log-normal distribution for these repair times have the following input values:

Table 7-4. Corrective Maintenance Activity Clock Time Distributions.

\begin{tabular}{|l|c|c|c|c|c|}
\hline CM Activity & $\begin{array}{c}\text { Relative Frequency } \\
\text { (Mean Value) } \\
\left(x_{n}\right)\end{array}$ & $\begin{array}{c}\text { Log-Mean } \\
\alpha\end{array}$ & $\begin{array}{c}\text { Log-S. D. } \\
\beta\end{array}$ & $\begin{array}{c}5^{\text {th }} \\
\text { Percentile } \\
(\text { hrs.) }\end{array}$ & $\begin{array}{c}\mathbf{9 5}^{\text {th }} \\
\text { Percentile } \\
(\text { hrs. })\end{array}$ \\
\hline 3TCNEL $(n=1)$ & $48.5 \%$ & 5.08 & 0.56 & 64 & 400 \\
\hline 5TCISA $(n=2)$ & $48.5 \%$ & 4.89 & 0.56 & 71 & 284 \\
\hline 5TCIEL $(n=3)$ & $3 \%$ & 5.56 & 0.56 & 102 & 638 \\
\hline
\end{tabular}

\subsubsection{Ventilation System Failure Frequency}

Ventilation system failure frequency is estimated from the Barton and Bingham (1999) database which states the 1997 ventilation system availability for the AY / AZ tank farm is $99.997 \%$. In the pedigreed database, availability is defined as (total time - down time) / total time $\times 100$, where total time is the length of time tracked for the ventilation system exhauster fans and down time represents all periods when none of the exhauster fans are running. Using this value assumes that ventilation system failure is equivalent to primary ventilation system failure, and primary system ventilation failure is determined by the exhauster fan's status. This neglects failures in the annulus ventilation system or ancillary components, such as the condensate cooling systems. This is a best-estimate assumption justified by the discussion in Section 3-1, which shows that the supernatant can be cooled effectively by evaporation if the DST headspace temperature can be maintained (Figure 3-1).

Availability can be written in terms of failure rate, $\lambda$, and repair rate, $\mu$ :

$$
A=\frac{\mu}{\lambda+\mu}
$$


where the failure rate is the inverse of the mean time to failure and the repair rate is the inverse of the mean time to repair. This expression neglects down time due to preventative maintenance. Solving for the failure rate $\lambda$ gives

$$
\lambda=\mu\left(\frac{1}{\mathrm{~A}}-1\right)
$$

This equation was evaluated using the following assumptions:

- The mean time to repair $(1 / \mu)$ is uniform on the interval between $4 \mathrm{hrs}$ and 24 hours, and

- The unavailability $(1-\mathrm{A})$ is equal to $10^{\alpha}$, where $\alpha$ is uniform on the interval between -4.0 and -5.0 .

The mean time to repair is reflective of the restoration times listed in the WFD RAM study. The distribution for system unavailability incorporates the 1997 value $(1-0.99997)$ as the expected value, but considers a factor of 3 uncertainty about the expected value. In effect, the system unavailability is somewhere between $1.0 \times 10^{-4}$ and $1.0 \times 10^{-5}$ to consider the uncertainty associated with applying a lone data point to calculate an annual frequency. A Monte Carlo simulation was performed to obtain a failure rate output distribution. Results show a median value of $0.02 / \mathrm{yr}$ and $90 \%$ of the 1000 trials falling between $0.006 / \mathrm{yr}$ and $0.09 / \mathrm{yr}$. A continuous distribution for $\lambda$ was found by curve fitting to 30 random deviates from the simulation. A satisfactory fit per the chi-square test $(p$-value $=0.49$ ) was found to be a log-normal distribution with log-mean equal to -3.61 and $\log$-standard deviation equal to 0.782 .

\subsubsection{Tank Bump Conditions Frequency for LCO and Current Conditions}

Finally, the annual tank bump frequency due to random failures is calculated by substituting equation (7-4) into equation (7-2):

$$
F_{R}=f_{R}\left(1-x_{r s}\right) \sum_{n=1}^{3} x_{n}\left[1-\log \text {-normal }\left(t_{a}, \alpha_{n}, \beta_{n}\right)\right]
$$

Because many of the variables in this equation are either stochastic or uncertain, a distribution for $F_{R}$ was calculated assuming the following input distributions:

- The relative frequency of the CM activity 5 TCIEL is uniform between $3 \%$ and $10 \%$ and the other two $\mathrm{CM}$ activities are equally likely,

- The time to heat up to saturated conditions, $t_{h}$, is uniform between 25 and 35 days,

- The time to discover and initiate recovery from loss of ventilation, $t_{s}$, is uniform on the interval between 1 and 10 days, 
- The fraction of events that require corrective maintenance rather than restoration is uniform between $10 \%$ and $20 \%$, and

- $\quad$ Per the discussion in the previous section, $f_{R}$ is log-normal with log-mean equal to -3.61 and $\log$-standard deviation equal to 0.782 . This distribution was obtained assuming the current conditions, but it is applied to LCO conditions nevertheless.

After 1,000 Monte Carlo trials, the resulting distribution for the annual frequency of tank bump conditions due to random failures shown mean equal to $8 \times 10^{-5} / \mathrm{yr}$, with $78 \%$ of the output trials falling below $1 \times 10^{-4} / \mathrm{yr}$. This range can be attributed almost entirely to uncertainty in the time to self-heat to saturated conditions. The calculated reverse cumulative distribution function for tank bump frequency is shown in Figure 7-1. As an intermediate step in the tank bump frequency calculation, the failure to repair probability was calculated as well. The mean value for the failure to repair probability (or frequency per demand) was $2.2 \times 10^{-3} / \mathrm{d}$.

This calculation was repeated for the current waste conditions. That is, the time to heat up to saturated condition was changed to uniform on the interval between 80 and 100 days, with all other input distribution held constant. After 1,000 Monte Carlo trials, the resulting distribution had a mean of $6 \times 10^{-8} / \mathrm{yr}$ and no trials fell above $1 \times 10^{-6} / \mathrm{yr}$, which indicates that tank bumps are beyond extremely unlikely for the current conditions.

\subsection{LOSS OF OFF-SITE POWER FREQUENCY}

Loss of off-site power (LOSP) events at the tank farms have been considered previously. Existing work is cited here to show that a LOSP event lasting long enough to cause a tank bump is beyond extremely unlikely. In particular, the TWRS FSAR Sections 3.3.2.1.5 and 4.5.1.4 conclude that loss of electrical power to TWRS for 30 days is beyond extremely unlikely.

In support of the K Basin SAR, Shultz (1994) analyzed power loss data for the 200 Area Tank Farms. Shultz (1994) reviewed site unusual occurrence reports covering the years 1972 to 1992 and generated an extreme value plot of 200 Area power loss data. This plot showed the relationship between power outage duration and annual frequency. Specifically, the following values were reported for annual frequency: 0.25 for 1 hour, 0.067 for 4 hours, 0.01 for 24 hours, and 0.005 for 48 hours. On a cumulative probability scale for an extreme value distribution, Shultz (1994) drew a straight line through these data points and extrapolated to 1000 hours, where the annual frequency is $1.25 \times 10^{-4} / \mathrm{yr}$. Shultz (1994) does not endorse the use of this value, however, and goes on to state it would erroneous to do so because no effort was made to account for repairs that would limit the frequency of long duration events.

LOSP events have also been described for the Hanford site by combining Hanford tank farm data with typical nuclear power plant data from NUREG/CR-5496. Since generic, long-duration LOOP event frequencies are dominated by severe weather events, their application to the Hanford site is undoubtedly conservative, given the relatively placid conditions in the Columbia River Valley. In roughly 1500 site-years of commercial nuclear power operation, only the 1992 Turkey Point LOOP event following Hurricane Andrew lasted over five days. In rough terms, 
Figure 7-1. Calculated Reverse Cumulative Distribution for Tank Bump Frequency.

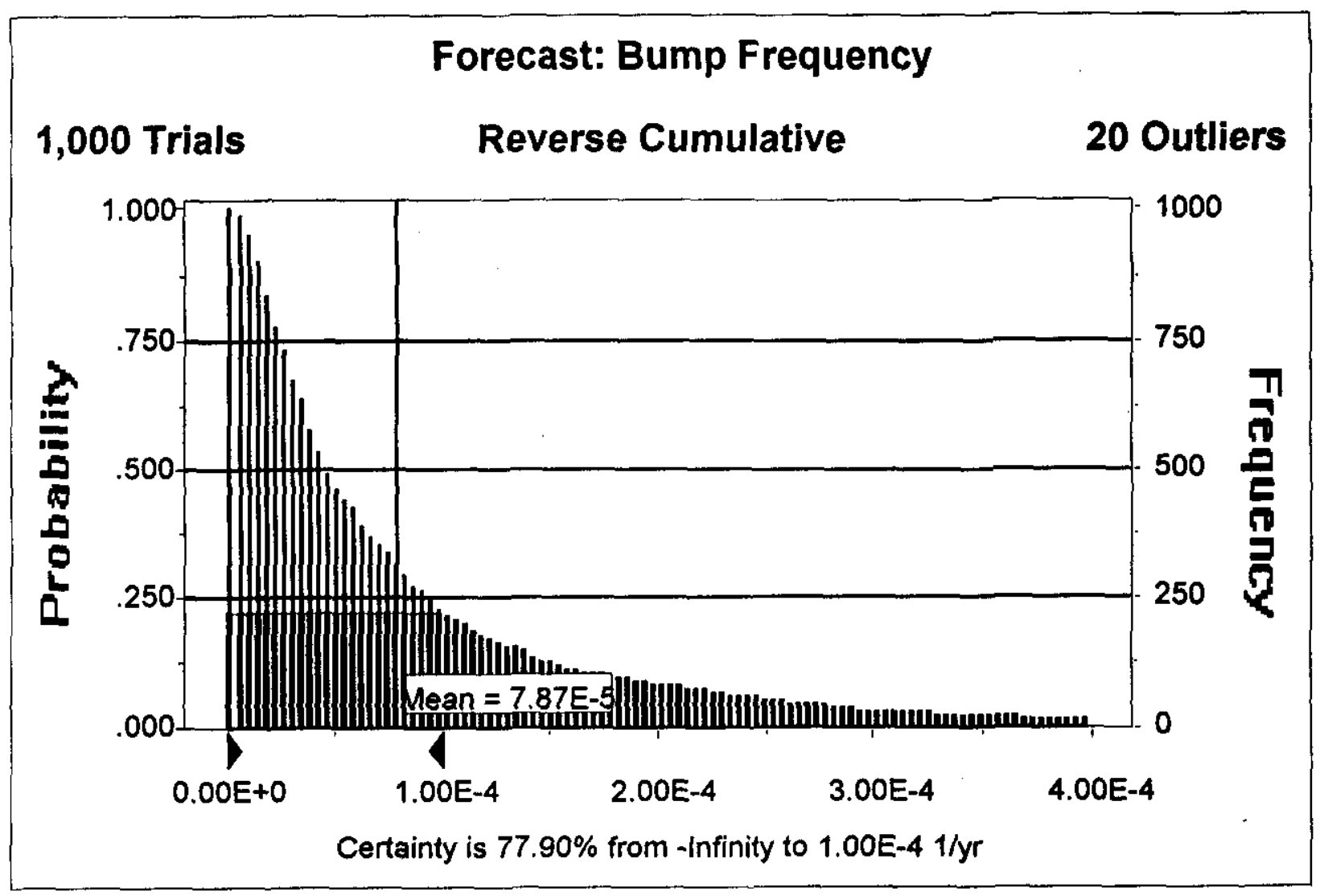


this is a $7 \times 10^{-4}(1 / 1500)$ annual frequency of a five day LOSP. Recently, LOSP frequency estimates were presented to the DOE, based on the data of NUREG/CR-5496 (Hunt 2000) and tank farm data. LOSP frequency as a function of duration is shown in Figure 7-2. Hunt (2000) states that this curve should be used with caution for events past 24 hours, but also notes that the Turkey Point event is in reasonable agreement with the other data points. The site-specific LOSP frequency for long duration events is less than can be expected for the nuclear power industry as a whole because severe weather is much less of an issue at the Hanford site. The frequencies shown by Hunt are generally smaller than those reported by Shultz (1994).

Although the LOSP curve does not extend out to the time available for recovery (30 days), a value at 7 days of $8 \mathrm{E}-5$ per year is adopted here for conservatism. Within a period of 30 days, recovery actions in response to an extended LOSP are plausible, and the LOSP frequency should be adjusted by the likelihood of initiating and maintaining emergency AC power supplies. For example, a diesel generator with a failure rate of $0.005 / \mathrm{hr}$ (Carlson 1999) and mean time to repair of 8 hours has a long term availability of $96 \%$, thereby making the LOSP contribution to tank bump frequency not credible.

\subsection{LIGHTNING STRIKES}

Lightning strikes have been considered previously in Cowley and Stepnewski (1994), Buck (1993), and Zach (1996). Cowley and Stepnewski (1994) considered the probability of a lightning strike in a given space during a given time interval at the site. An empirical formula for this parameter is

$$
\mathrm{LS}=10^{-6} \mathrm{~A} \Delta \mathrm{t}\left(1 / \mathrm{m}^{2}-\mathrm{yr}\right)
$$

where LS is the number of lightning strikes per square meter per year, $\mathrm{A}$ is area in square meters, and $\Delta t$ is the time in years. For example, because the surface area above a DST is $500 \mathrm{~m}^{2}$, the annual lightning strike frequency is about once every 2000 years. Assuming there are 161 large tanks (17 smaller tanks can be neglected), one tank is struck every 13 years or so. Total tank farm area is roughly 10 times the total tank area, so lightning strikes a single tank farm roughly once a year; anecdotes from operations personal support this estimate.

In Zach (1996), a strike frequency of $0.06 / \mathrm{km}^{2} / \mathrm{yr}$ is reported, which is about $6 \%$ of the value reported by Cowley and Stepnewski (1994).

To evaluate the annual frequency of loss ventilation due to a lightning strike, the relevant area of the ventilation system and its ancillary functions needs to be determined. In Cowley and Stepnewski (1994), ventilation systems are identified as farm-specific, along with control panels/enclosures, power supplies, and buildings/structures. A conservative assumption is that a strike leads to a loss of that piece of equipment. Only the strike area for the AZ / AY farm is considered here, per the results of Section 6.0 which show that these are the DST susceptible tank bumps. Because ventilation system components provide a strike area much smaller than the area above the DST, the strike area is estimated to roughly $10 \mathrm{~m}^{2}$, which means that annual loss of HVAC in either tank due to a lightning strike is $1 \times 10^{-5}$ using the 
Figure 7-2. Loss of Off-Site Power (LOSP) Frequency of Duration.

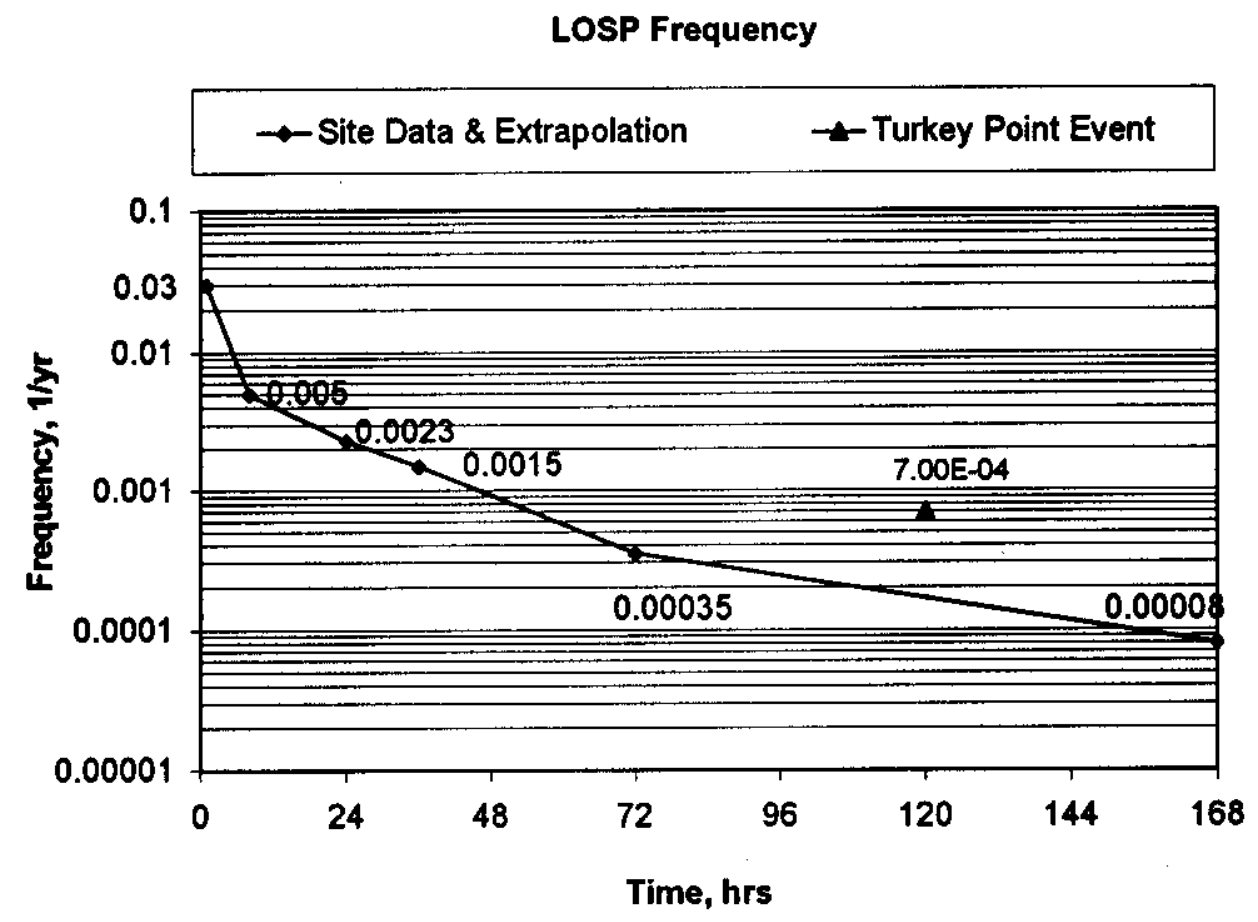


Cowley and Stepnewski (1994) annual strike frequency. Of course, this exceeds the value predicted by using the Zach (1996) strike frequency by a factor of 16.

Unlike LOSP or seismic events, however, recovery actions, restoration times and repair times are independent of the initiator. If a lightning strike leads to a ventilation outage, the failure to recover probability during the available interval is about the same as that computed for random failures. Therefore, lightning strikes can be neglected as an initiator because random failures occur with much greater frequency $\left(0.1\right.$ to $0.01 / \mathrm{yr}$ vs. $\left.1 \times 10^{-5} / \mathrm{yr}\right)$, yet lightning strikes and random failures result in comparable failure to recover probabilities.

\subsection{SEISMIC EVENTS}

A seismic performance goal is $10^{-4}$ annual probability that the tanks are damaged to the extent that containment is compromised (DOE 1994). Seismic fragility analyses of both SSTs and DSTs indicate that this performance goal is met with some margin.

The annual frequency of tank bump conditions caused by seismic events can be calculated by:

$$
F_{S}=\int_{g_{\text {low }}}^{g_{\text {up }}} p_{s}(g) P_{\text {trs }}\left(t_{a}, g\right) d g
$$

where $p_{s}(g)$ is the probability density function for the seismic hazard curve as a function of peak horizontal acceleration $\mathrm{g}$ (in units of $981 \mathrm{~cm} / \mathrm{s}^{2}$ ), and $P_{n r S}\left(t_{g}, g\right)$ is the failure to recover probability as a function of available time and the peak horizontal acceleration $\mathrm{g}$. The limits of integration are practical upper and lower bounds defined as follows: the lower limit is some acceleration below which the consequences of a seismic event are trivial, and the upper limit is some acceleration above which the annual frequency is negligible. The seismic hazard curve for the Hanford site given in Han (1996b) extends from about $0.05 \mathrm{~g}$ to $0.9 \mathrm{~g}$ and shows the following data:

Table 7-5. Seismic Hazard Curve Data for the Hanford Site.

\begin{tabular}{|c|c|}
\hline Acceleration (g) & Annual Frequency \\
\hline 0.05 & $1.00 \mathrm{E}-02$ \\
0.1 & $2.00 \mathrm{E}-03$ \\
0.2 & $5.30 \mathrm{E}-04$ \\
0.3 & $1.80 \mathrm{E}-04$ \\
0.4 & $1.00 \mathrm{E}-04$ \\
0.5 & $5.00 \mathrm{E}-05$ \\
0.6 & $3.00 \mathrm{E}-05$ \\
0.7 & $2.00 \mathrm{E}-05$ \\
0.8 & $1.50 \mathrm{E}-05$ \\
0.9 & $1.00 \mathrm{E}-05$ \\
\hline
\end{tabular}


The annual frequency data shown here can be interpreted as a probability density function by noting that the data is really the reverse cumulative distribution function (one minus the cumulative distribution function) for the occurrence of some horizontal acceleration $\mathrm{g}$ during the course of a year. An excellent fit to the data $\left(r^{2}=0.995\right)$ is given by $F=b g^{a}$, where $\mathrm{b}=9.53 \times 10^{-6} / \mathrm{yr}$ and "a" equals -2.367 ; in this expression, the peak horizontal acceleration, $\mathrm{g}$, must be dimensionless in terms of some fraction of the acceleration of gravity. On the interval between $g=0.05$ and $g=0.90$, the cumulative distribution, $P_{s}$, is

$$
P_{s}=1-F=1-b g^{2}
$$

and the probability density function, $\mathrm{p}_{\mathrm{s}}$, can be written,

$$
\mathrm{p}_{\mathrm{s}}=\frac{\mathrm{d} \mathbf{P}_{\mathrm{s}}}{\mathrm{dg}}=-\mathrm{ba} \mathrm{g}^{(\mathrm{a}-1)}
$$

In the interval of interest, a good approximation to the probability density function for the seismic hazard curve is given $p_{s}=2.256 \times 10^{-5} \mathrm{~g}^{-3.367}$. Note that this is not a general p.d.f. for the seismic hazard curve, but is an approximation that is valid only in the limited range of interest.

The failure to recover probability as a function of $g$ must now be specified. In general, this is a lengthy process that requires estimates of the seismic fragility of the individual components in the ventilation system. This approach is not warranted here and a simple failure to recover probability curve is developed based on two assumptions: first, the failure to recover probability at relatively small peak accelerations is no worse than the failure to recover probability for random failures (roughly $2.2 \times 10^{-3}$ per demand for $\mathrm{LCO}$ ), and second, at large peak acceleration values, the failure to recover probability approaches one because the necessary repairs would take months, for reasons explained below.

With respect to the hazard curve shown above, a reasonable assumption is that as $\mathrm{g}$ approaches the high end of the range (say above $0.6 \mathrm{~g}$ ), the damage is extensive enough that repairs would require pit and/or tank access and take many months to enact (see "Major Mechanical Repairs" in Table 7-3). Various estimates for DST fragility have consistently placed the onset of DST failure at or slightly above $0.8 \mathrm{~g}$ (Han 1996a and 1996b). According to the DELPHI expert panel of Han (1996b), at an acceleration level of $0.8 \mathrm{~g}$, wall shear damage, or so-called "walking", dome cracking and leakage can be expected. The same panel deemed a $1.5 \mathrm{~g}$ acceleration limiting for the dome. A probabilistic fragility analysis developed for tank 241-SY-101 gross leakage from the tank could be expected at a mean value of $2.7 \mathrm{~g}$ and high confidence, a low probability (HCLPF) value of $1.05 \mathrm{~g}$ (Wesley and Nakiki 1992). Wesley and Nakiki (1992) used a somewhat less conservative definition of tank failure than did the DELPHI panel of Han (1996b) (gross leakage vs. onset of failure), but the two results are essentially consistent.

The failure to recover probability curve is then some S-shaped function with the following characteristics: 
- At low values of $\mathrm{g}$, in the range between 0.05 and $0.2 \mathrm{~g}, \mathrm{P}_{\mathrm{nr}}$ is on the order of $2.2 \times 10^{-3}$ because seismic events will not result in repair times any different than random failure events,

- At intermediate values, in the range between 0.2 and $0.6 \mathrm{~g}, P_{\mathrm{nrS}}$ rapidly increases from $2.2 \times 10^{-3}$ to values somewhere between 0.1 and 0.5 , as the probability of extensive damage that prevents recovery becomes credible but not necessarily likely, and

- At high values, in the range between 0.6 and $0.9, P_{\text {nrs }}$ approaches unity.

Any number of functions fulfill these requirements, but the following one is used here:

$$
P_{n r S}(g)=\operatorname{Max}\left[2.2 \times 10^{-3}, \operatorname{Normal}(g, \mu, \sigma)\right]
$$

where normal is the cumulative probability for a normal distribution with mean $\mu$ and standard deviation $\sigma$. This function is plotted below in Figure 7-3 with mean equal to 0.6 and standard deviation equal to 0.05 .

The tank bump frequency due to seismic events is then given the integral,

$$
F_{S}=\int_{g=0.05}^{g=0.90}-(\mathrm{b} \mathrm{a}) \mathrm{g}^{\mathrm{a}-1} \operatorname{Max}\left[2.2 \times 10^{-3}, \operatorname{Normal}(\mathrm{g}, \mu, \sigma)\right] \mathrm{d} \mathrm{g}
$$

which can be evaluated by any convenient numerical scheme. An uncertainty analysis was performed with the following input distributions:

- To reflect typical uncertainties associated with seismic hazard curves, the parameter $\left(-\mathrm{b}\right.$ a) was triangular on the interval between $1.00 \times 10^{-5} / \mathrm{yr}$ and $5 \times 10^{-5} / \mathrm{yr}$, with $2.25 \times 10^{-5} / \mathrm{yr}$ as the most likely value,

- To reflect the uncertain nature of the failure to recover probability, the mean of the cumulative normal distribution was uniform between 0.4 and $0.6 \mathrm{~g}$, and

- The standard deviation of the cumulative normal distribution was uniform between 0.05 and $0.10 \mathrm{~g}$.

The resulting distribution for $F_{S}$, the frequency of tank bumps induced by seismic events, is shown below in Figure 7.4. The distribution has mean equal to $7 \times 10^{-5} / \mathrm{yr}$ with $82 \%$ of the trials falling between $1 \times 10^{-4} / \mathrm{yr}$ and $2 \times 10^{-5} / \mathrm{yr}$. The mean value of this distribution is hardly effected by changes in the failure to repair frequency, which means that the large magnitude earthquakes determine the seismic bump frequency. 
Figure 7-3. Failure to Repair Function, $\mathbf{P}_{\mathrm{nrS}}(\mathrm{g})$.

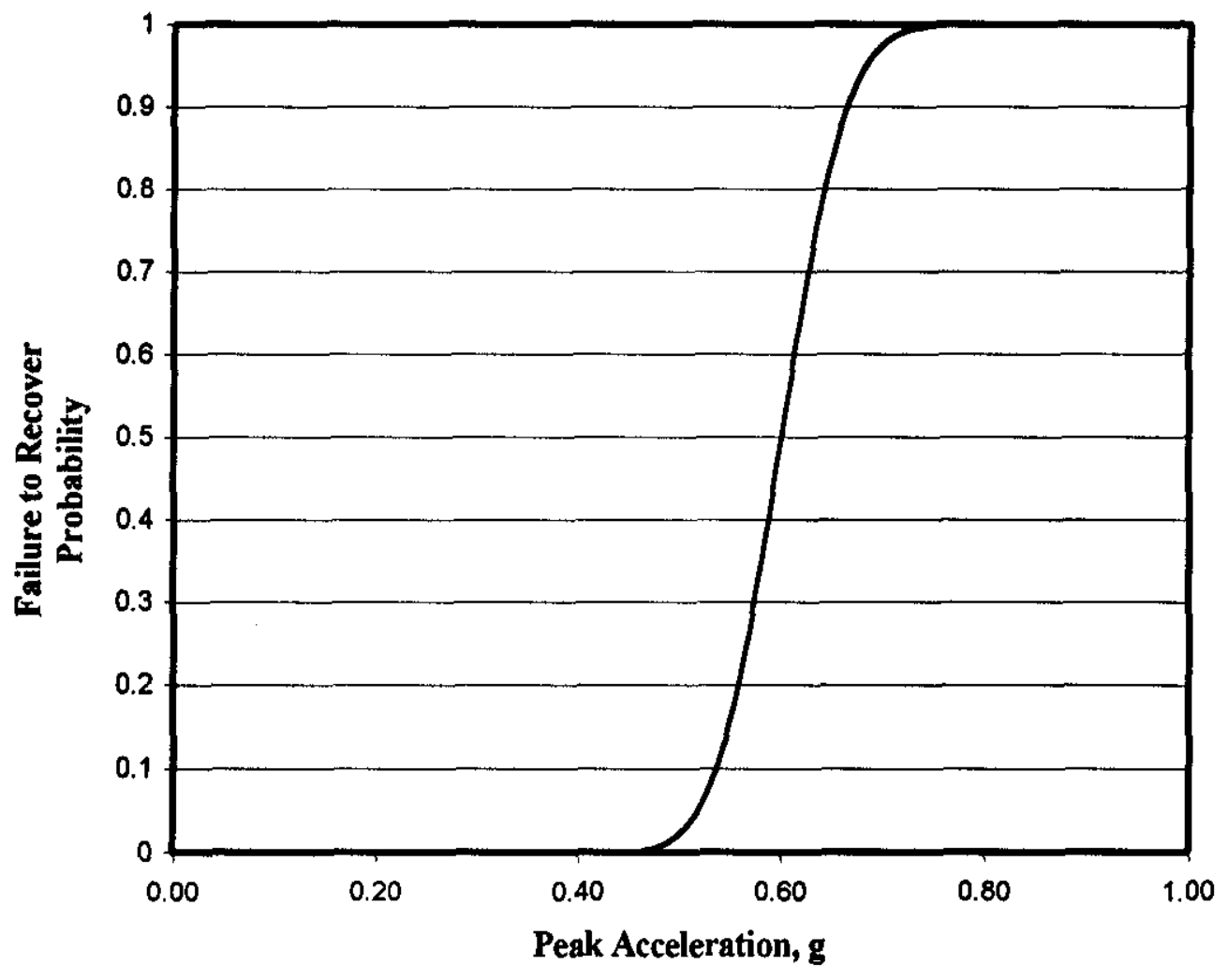




\section{RPP-6213 REV 0}

Figure 7-4. Distribution for the Frequency of Tank Bumps Induced by Seismic Events.

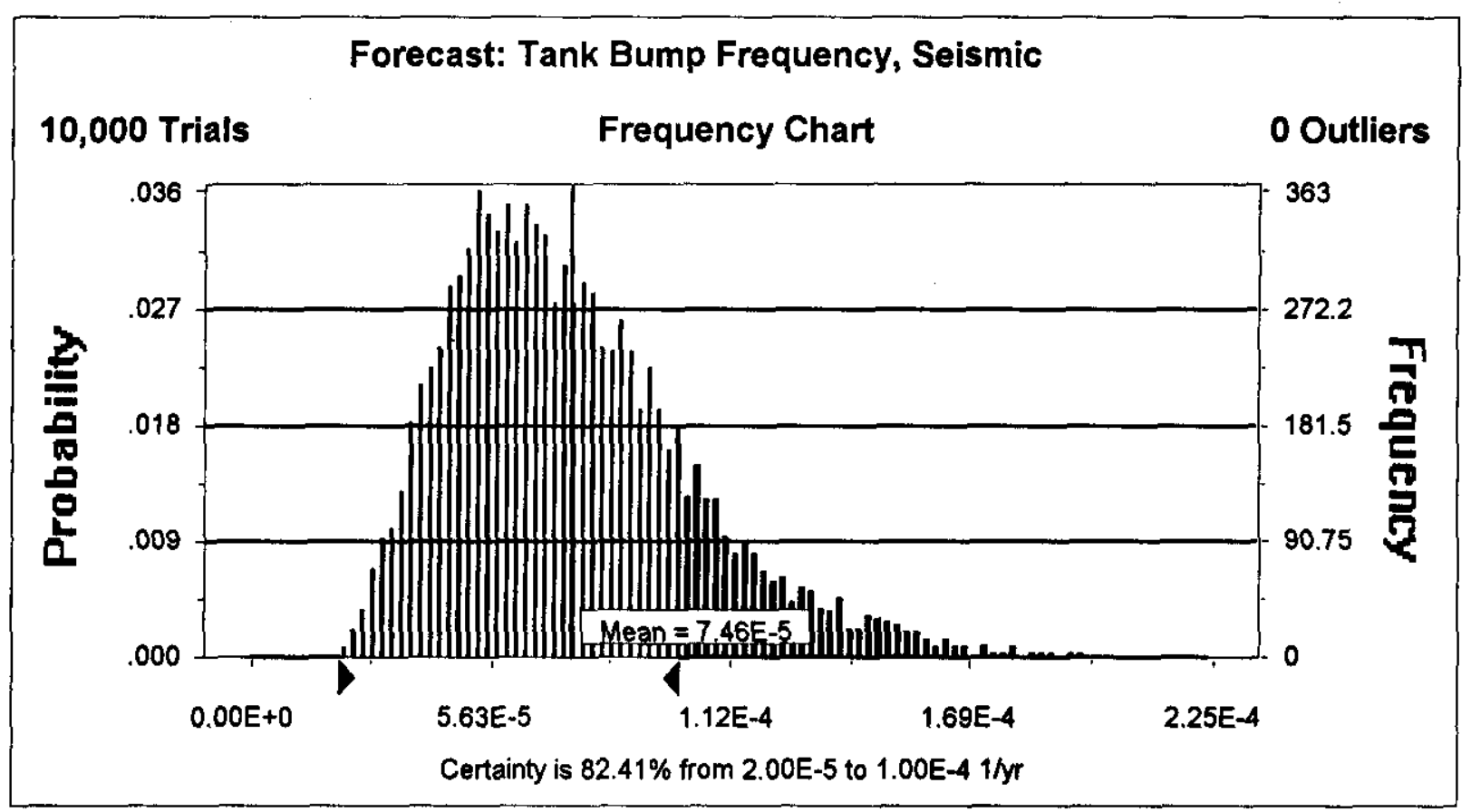




\subsection{CONCLUSIONS}

Tank bump annual frequency is best described as "extremely unlikely", based on the foregoing calculations. Random failures were considered, along with three external initiating events: loss of off-site power, seismic events, and lightning strikes. With respect to each of these four contributors to tank bump frequency, the following can be noted:

- Under the current conditions, tank bumps be can categorized as beyond extremely unlikely; the estimated mean frequency is much less than $1 \times 10^{-6} / \mathrm{yr}$ because the available recovery times far exceed,

- Without ventilation controls, i.e., for tanks operating at the LCO limit, tank bump condition frequency would be about $1 \times 10^{-4}$, which is the dividing line between unlikely and extremely unlikely; a consequence calculation is performed in the next section to determine if this frequency is unacceptably high,

- The contribution from seismic events must also be categorized as extremely unlikely: the mean frequency of a bump due to this initiator is $7 \times 10^{-5} / \mathrm{yr}$, with a $82 \%$ confidence that the frequency is between $1 \times 10^{-4} / \mathrm{yr}$ and $2 \times 10^{-5} / \mathrm{yr}$,

- The loss of off-site power initiator has a frequency that is beyond extremely unlikely, and

- The lightning strike initiator does not result in recovery times any different that random failure, but has a much lower frequency.

Tank bumps are not an important contributor to current tank farm risk. Tank bump conditions develop only after a very long period. Equipment failures due to random causes or small, relatively frequent seismic events can be remedied in much less time than 80 to 100 days expected as a time to bump for the AZ / AY tank farm. The major contribution to tank bump frequency as calculated here stems from large magnitude seismic events, but these events are likely to create site consequences that are much worse than a tank bump. 
RPP-6213 REV 0

This page intentionally left blank.

FAI/00-14, Rev. 0 


\subsection{CONSEQUENCE EVALUATION}

Here we consider consequences of a tank bump that occurs during off-normal conditions of storage, i.e., without retrieval actions. Due to the long time for waste heatup after loss of power in such circumstances, such events are expected to be extremely unlikely, as shown in Section 7. Although screening in Section 6 ruled out all tanks, tank 241-AZ-102 is chosen as a representative tank for a single consequence evaluation because it has a combination of shortest time to saturation and a displacement figure-of-merit (equation (4-16)) greater than unity.

The consequence model employed is essentially that of Section 5 with modification to consider gas flows from the tank headspace. This allows the conservative adiabatic headspace compression assumption to be relaxed, and ultimately allows attenuation in the headspace to be considered. Also, aerosol models are added, as are representations of pump and sluice pit regions. This is accomplished by incorporating the model from Section 5 into the HADCRT code, as described next.

\subsection{THE HADCRT CODE}

The HADCRT code (Malinovic et al. 2000) is an integrated model for considering storage tank thermal-hydraulic and accident phenomena, such as deflagration, detonation, and the potential for fission product release. HADCRT accounts for generic phenomena such as gas and aerosol transport between regions, and heat transfer to structures, including evaporation/condensation. Generic phenomenological capabilities include:

- Multiple compartment representation,

- Pressure-driven and counter-current gas flows,

- Gas and aerosol transport between compartments,

- Vapor-aerosol equilibrium,

- Aerosol deposition due to gravitational sedimentation, impaction, etc., and

- Heat transfer to structures.

Routines containing phenomenology specific to an accident or thermal-hydraulic scenario are used to provide rates-of-change for the generic routines listed above, for example, vapor-phase combustion followed by blowdown and entrainment of aerosols. Accident-specific routines provides sources of mass, such as products of combustion or entrained aerosols from liquid surfaces and deposited solid particulate, or energy, as in the heat of reaction by hydrogen combustion.

The tank bump phenomena described in this section were coded and added to the HADCRT code. HADCRT integrates the rates-of-change over the course of the bubble cluster rise to obtain position, volume of gas and liquid, vapor pressure, and temperature as a function of time, 
as described in equations (5-1) through (5-9) and (5-17) and (5-25). Temperature and pressure in the headspace are tracked by generic models during bubble cluster rise, so as to capture the feedback between bubble cluster dynamics and the headspace conditions during compression.

Vapor and entrained aerosols passing through the top of the supernatant are a source of mass and energy to the headspace. When the bubble cluster center reaches the supernatant surface, which has swollen per equation (5-6), the aerosol release equations (5-12) through (5-14) define the mass of material released to the headspace. These sources are used in turn to determine pressure, temperature, and airborne aerosol mass as a function of time.

Effects of headspace inflow/outflows and heat transfer to structures during and after the bump. Coding details are left for Appendix F.

\subsection{HADCRT INPUT FOR 241-AZ-102 CASE}

Best-estimate consequences of a series of tank bumps in 241-AZ-102 are considered here to exemplify consequences. The HADCRT model for this case contains four volumes or regions: the tank headspace, a region representing four sluice pits, the center pump pit, and the environment. Normal flowpaths between the environment and the headspace are the filtered 8" diameter inlet and the filtered 20" diameter. Tank configuration is given in Section 3.4. Sluice pit covers lift at a pressure differential of $13.8 \mathrm{kPa}$ and pump pit covers lift at a pressure differential of $17 \mathrm{kPa}$. Other inputs are shown in Table 8-1.

For the tank bump model, 241-AZ-102 inputs are as follows: convective layer height $=7.0 \mathrm{~m}$, non-convective waste temperature $=385 \mathrm{~K}$, supernatant temperature $=100^{\circ} \mathrm{C}$, and supernatant volume $=2859 \mathrm{~m}^{3}$. Other parameters important to bump size are the volume of gas releases during the buoyant displacement, the initial mass of liquid in the buoyant parcel, the time between bumps, and the number of bumps. The volume of gas for 241-AZ-102 is computed using equation (5-10), assuming $P_{\mathrm{NCL}}=1.8 \mathrm{~atm}, \alpha_{\mathrm{NB}}=0.262, \mathrm{~h}_{\mathrm{NCL}}=96 \mathrm{~cm}, \rho_{\mathrm{CL}}=1100 \mathrm{~kg} / \mathrm{m}^{3}$, and $\tau_{\mathrm{y}}=100 \mathrm{~Pa}$. This results in a gas volume of $\mathrm{V}_{\mathrm{b}}(0)=8 \mathrm{~m}^{3}$. With a non-convective layer density of $1490 \mathrm{~kg} / \mathrm{m}^{3}$, the initial amount of liquid plus suspended solids in the buoyant parcel is $34,000 \mathrm{~kg}$. Average time between bumps is given by equation (5-12):

$$
t_{B D}=\frac{V_{b}(0)}{G_{g} A_{T} h_{N C L}}
$$

The volume of noncondensable gas generated per unit volume of non-convective material per unit time, $\mathrm{G}_{\mathrm{g}}$ is given by:

$$
\frac{G_{g}}{G_{0}}=\left(\frac{1}{0.15}\right)^{3}
$$

where the 0.15 value is the buoyant displacement model prediction in Table $4-2$ and $G_{0}$ is the value of $T_{b p}$ from Table 4-1, $0.19 \mathrm{gmol} / \mathrm{m}^{3} /$ day. This results in $G_{g}=1.02 \mathrm{~m}^{3} / \mathrm{m}^{3} /$ day after 
Table 8-1. Parameter Values for Consequence Analysis Example for Tank 241-AZ-102.

\begin{tabular}{|c|c|}
\hline \multicolumn{2}{|c|}{ Waste and Headspace rarameters } \\
\hline$V_{b}(0)=6.54 \times 10^{-8} \mathrm{~m}^{3}$ & $\begin{array}{l}\text { Initial volume of representative bubble based on } 5.0 \mathrm{~mm} \\
\text { bubble diameter. }\end{array}$ \\
\hline$V_{b}(0)=8 m^{3}$ & $\begin{array}{l}\text { Initial total volume of bubbles released from sludge and used } \\
\text { in ratio } V_{b} / V_{b}(0) \text {, parameter variation. } 8 \mathrm{~m}^{3} \text { is a best-estimate } \\
\text { case. }\end{array}$ \\
\hline$V_{l}=2859 \mathrm{~m}^{3}$ & Volume of convective layer. \\
\hline$V_{\mathrm{hs}}(0)=1800 \mathrm{~m}^{3}$ & Initial volume of headspace. \\
\hline$H(0)=7.0 \mathrm{~m}$ & Initial depth of convective layer. \\
\hline$m_{\ell}(0)=34,000 \mathrm{~kg}$ & Initial non-convective layer mass in parcel. \\
\hline $\mathrm{T}_{\ell}(0)=115^{\circ} \mathrm{C}$ & Initial parcel temperature. \\
\hline $\mathrm{P}_{\mathrm{hs}}(0)=1.012 \times 10^{5} \mathrm{~Pa}$ & Initial headspace pressure. \\
\hline$T_{h s}(0)=100^{\circ} \mathrm{C}$ & Headspace initial temperature. \\
\hline$X_{\text {st }}(0)=95 \%$ & Headspace steam mole fraction. \\
\hline \multicolumn{2}{|c|}{ Flow Path Parameters } \\
\hline$A_{\text {in }}=0.03 \mathrm{~m}^{2}$ & $8 "$ inlet line. \\
\hline$A_{\text {out }}=0.20 \mathrm{~m}^{2}$ & $20^{\prime \prime}$ outlet line. \\
\hline$A_{\text {leak }}=5 \times 0.00133 \mathrm{~m}^{2}$ & Leakage around $42^{\prime \prime}$ risers. \\
\hline$\Delta \mathrm{P}_{\text {HEPA }}=35,600 \mathrm{~Pa}$ & HEPA failure $\Delta \mathrm{P}$, crediting vent path pressure losses. \\
\hline$C_{e}=0.5$ & Flow coefficient for inlet and outlet paths. \\
\hline $\begin{aligned} V_{\text {pit }}= & 4 \times 10 \mathrm{~m}^{3} \\
& +1 \times 20 \mathrm{~m}^{3}\end{aligned}$ & Sluice pit and center pit volumes. \\
\hline $\mathrm{T}_{\text {pit }}(0)=75^{\circ} \mathrm{C}$ & Pit initial temperature. \\
\hline $\mathrm{P}_{\text {pit }}(0)=1.012 \times 10^{5} \mathrm{~Pa}$ & Pit initial pressure. \\
\hline \multicolumn{2}{|l|}{ (1. Property Values. } \\
\hline$\rho_{\ell}=1100 \mathrm{~kg} \mathrm{~m}^{-3}$ & Density of convective layer. \\
\hline$\mu_{\mathrm{g}}=1.2 \times 10^{-5} \mathrm{~kg} \mathrm{~m}^{-1} \mathrm{~s}^{-1}$ & Viscosity of bubble gas (vapor). \\
\hline$\rho_{g}=0.6 \mathrm{~kg} \mathrm{~m}^{-3}$ & Density of bubble gas (vapor). \\
\hline$\sigma=0.059 \mathrm{~kg} \mathrm{~s}^{-2}$ & Surface tension of supernatant. \\
\hline $\mathrm{D}=9.2 \times 10^{-5} \mathrm{~m}^{2} \mathrm{~s}^{-1}$ & Bubble gas/water vapor diffusion coefficient. \\
\hline
\end{tabular}


converting units. Substituting into the equation for average time between bumps gives

0.48 hours. Since there are about $100 \mathrm{~m}^{3}\left(\alpha_{\mathrm{NB}} \mathrm{A}_{\mathrm{r}} \mathrm{h}_{\mathrm{NCL}}\right)$ of gas in the non-convective layer, there will be 12 bumps spaced at roughly half-hour intervals.

\subsection{1-AZ-102 TANK BUMP CONSEQUENCE PHYSICAL RESULTS}

Results of the 241-AZ-102 tank bump calculation are shown in Figures 8-1 and 8-2, which provide short- and long-term histories for headspace temperature and pressure, and the aerosol distribution. Looking at short-term results, the pressure attained during a bump is about 3 psig, which is sufficiently large to not credit the HEPA filters as assumed. Temperature and pressure decay immediately following a bump due to forced flow and natural circulation flow to the environment. The natural circulation flow path is down through the damaged inlet line and up through the damaged outlet line.

In the first bump, about $1.5 \mathrm{~kg}$ of aerosol are released, of which somewhat more than $10 \%$, about $0.17 \mathrm{~kg}$, is forced into the environment, so that $1.3 \mathrm{~kg}$ are retained. During the half-hour interval between bumps, sedimentation in the tank accounts for about $0.5 \mathrm{~kg}$ depletion from the headspace and natural circulation flow removes about $0.05 \mathrm{~kg}$, so that about $0.75 \mathrm{~kg}$ aerosol remain when the second bump occurs. In the second and all successive bumps, about the same amount of aerosol is produced, but more aerosol is available in the headspace for release during short-term blowdown. Therefore, the cumulative release to the environment increases faster than linearly with the number of bumps, for this chosen bump interval of one-half hour. Also, as the aerosol concentration builds up, after each bump, the amount of sedimentation between bumps increases. Due to property variations, the average amount of aerosol released per bump is about $1.8 \mathrm{~kg}$.

After 12 bumps in this calculation, the entire non-convective layer has undergone buoyant displacement. Immediately following this last bump, the aerosol inventory is as follows: about $13.5 \mathrm{~kg}$ are settled in the tank, $3 \mathrm{~kg}$ are airborne in the headspace, and $5 \mathrm{~kg}$ are released to the environment, from a total source of about $21.4 \mathrm{~kg}$. Long-term natural circulation competes with settling, but only an additional $0.25 \mathrm{~kg}$ are released while the remainder of the $3 \mathrm{~kg}$ airborne settle. Overall, $5.2 \mathrm{~kg}$ are released to the environment and $16.2 \mathrm{~kg}$ are retained in the tank.

The present calculation considers one sequence of bumps that eventually displaces all nonconvective material. Sedimentation, noncondensable gas retention, and attainment of neutral buoyancy are required for a subsequent bump sequence. Another sequence of successive bumps can therefore repeat later in time after neutral buoyancy is again achieved, on a timescale consistent with buoyant displacement events.

\subsection{1-AZ-102 TANK BUMP RADIOLOGICAL AND TOXIC CHEMICAL CONSEQUENCES}

Mass of entrained material is converted to dose using the following method from Cowley et al. (2000): 
Figure 8-1. 241-AZ-102 Tank Bump Short-Term Results.
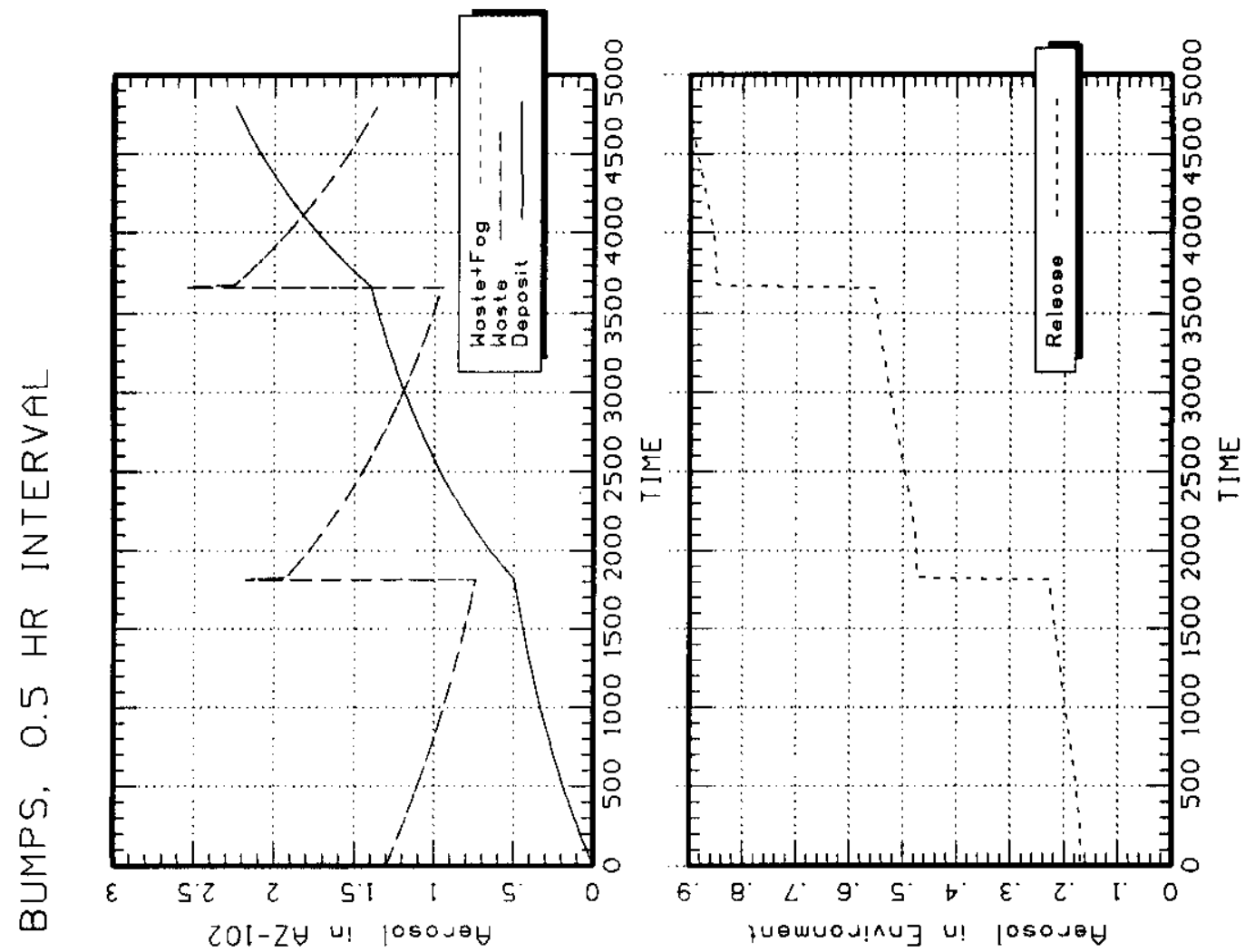

$\simeq$
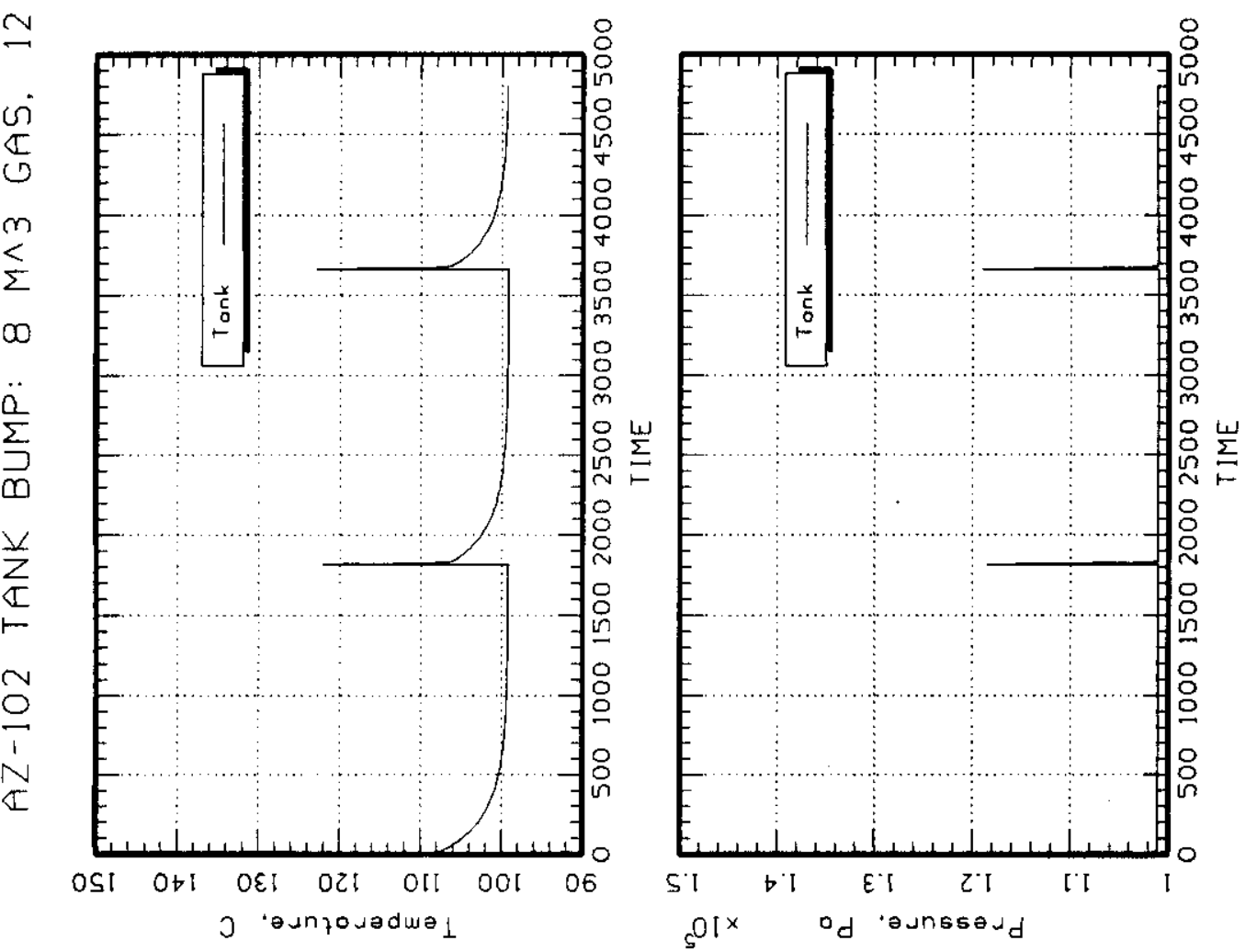


\section{RPP-6213 REV 0}

Figure 8-2. 241-AZ-102 Tank Bump Long-Term Results.

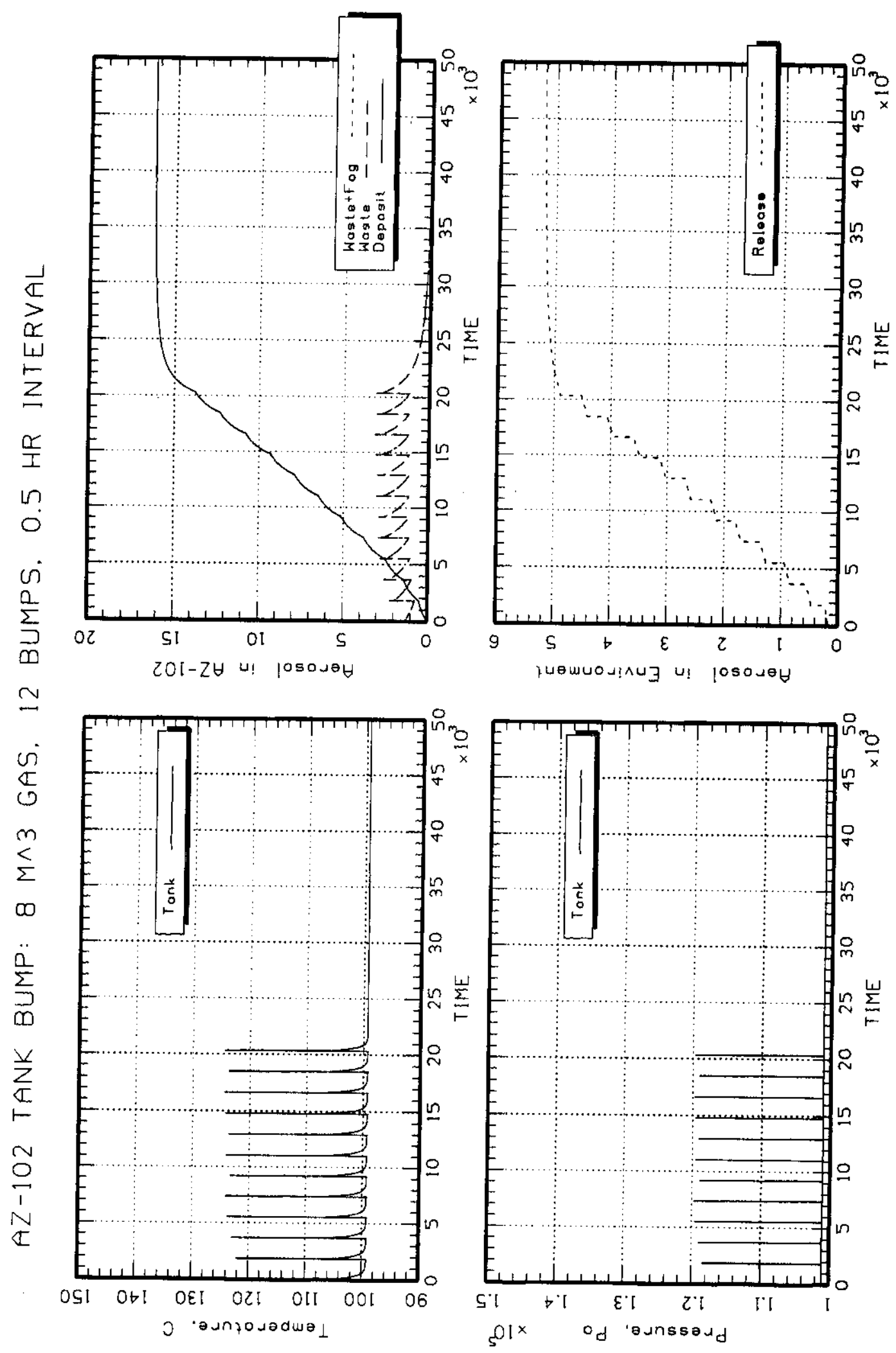




$$
\mathrm{D}=(\mathrm{Q})(\mathrm{ULD})\left(\frac{\mathrm{X}}{\mathrm{Q}}\right)(\mathrm{BR})
$$

where $\mathrm{D} \quad=$ Dose, Sv,

$\mathrm{Q} \quad=\quad$ Released quantity, $\mathrm{L}$,

ULD = Unit Liter Dose, Sv/L,

$\mathrm{X} / \mathrm{Q}=$ Atmospheric dispersion coefficient, $\mathrm{s} / \mathrm{m}^{3}$, and

$\mathrm{BR}=$ Breathing rate, $\mathrm{m}^{3} / \mathrm{s}$.

The released mass, $\mathrm{kg}$, calculated above can be converted into volume, $\mathrm{L}$, for waste solids and liquids. Then, parameter values from Cowley et al. (2000) as summarized in Table 8-2 may be applied.

Table 8-2. Radiological Consequence Factors (Cowley et al. 2000).

\begin{tabular}{|l|c|c|}
\hline \multicolumn{1}{|c|}{ Factor } & On-Site & Off-Site \\
\hline ULD, Sv/L & & \\
DST Solids & $1.07 \times 10^{5}$ & $1.84 \times 10^{5}$ \\
DST Liquids & $7.97 \times 10^{2}$ & $8.45 \times 10^{2}$ \\
\hline $\mathrm{X} / \mathrm{Q}, \mathrm{s} / \mathrm{m}^{3}$ & $5.58 \times 10^{-3}$ & $8.0 \times 10^{-6}(1)$ \\
\hline $\mathrm{BR}, \mathrm{m}^{3} / \mathrm{s}$ & $3.33 \times 10^{-4}$ & $3.33 \times 10^{-4}$ \\
\hline & (1) Calculated in equation (8-4).
\end{tabular}

For on-site dose, worker exposure at $100 \mathrm{~m}$ is taken for an 8-hour shift, so the $\mathrm{X} / \mathrm{Q}$ value of $5.58 \times 10^{-3} \mathrm{~s} / \mathrm{m}^{3}$ from Section 5.2 .3 of Cowley et al. (2000) applies. Similarly, the breathing rate is specified in Section 3.7 of the reference for light work.

For off-site dose, the methods of Section 5.2.3 of Cowley et al. (2000) are applied using values from Table 5-2 of the reference and approximating the release as an 8-hour event because $90 \%$ of the release occurs over a 6 -hour interval. Performing this evaluation,

$$
\frac{\log \left(1.74 \times 10^{-5}\right)-\log \left(\mathrm{X} / \mathrm{Q}^{8 \mathrm{hrs} .}\right)}{\log \left(1.74 \times 10^{-5}\right)-\log \left(1.47 \times 10^{-7}\right)}=\frac{\log (2 \mathrm{hrs} .)-\log (8 \mathrm{hrs} .)}{\log (2 \mathrm{hrs} .)-\log (8,760 \mathrm{hrs} .)}
$$

yielding $X / Q=8 \times 10^{-6} \mathrm{~s} / \mathrm{m}^{3}$. The light work breathing rate also applies off-site due to the short release duration.

Next, liters of solid and liquids released must be derived. In the first bump, non-convective solids are substantially diluted by supernatant: an initial release of $34,000 \mathrm{~kg}$ (liquid + solid) is increased to about $120,000 \mathrm{~kg}$ total in the rising parcel after entrainment (a calculation detail not plotted). But in later bumps, some solids now present in the supernatant are entrained as well, so 
that in the last bump of the series, the tank mixed-mean solid fraction applies. From Hu et al. (2000), waste properties are:

$\begin{array}{lccc} & \text { Density (kg/l) } & \text { Volume (kL) } & \mathbf{H}_{2} \mathbf{O}(\mathbf{w t} . \%) \\ \text { Convective } & 1.10 & 3,131 & 84 \% \\ \text { Non-Convective } & 1.49 & 394 & 56 \%\end{array}$

where dissolved salts are responsible for an $\mathrm{H}_{2} \mathrm{O} \%<100 \%$ in the convective layer. From Crea's et al. (2000) Table B3-1, the solids volume fraction in the non-convective layer is $17 \%$. Thus, the overall mixed mean solids volume fraction is $(17)(394) /(394+3131)=1.9 \%$. Noting that the released non-convective volume is $34,000 \mathrm{~kg} / 1.49(\mathrm{~kg} / \mathrm{L})=22.8 \mathrm{~kL}$ and the entrained volume is $86,000 \mathrm{~kg} /(1.1 \mathrm{~kg} / \mathrm{L})=78 \mathrm{~kL}$, the maximum fraction of entrained solids at the final bump is approximately

$$
\frac{(1.9)(78)+(17)(23)}{78+23}=5.3 \%
$$

The average density of released material is simply $(34,000+86,000) /(22,800+78,000)$

$=1.19 \mathrm{~kg} / \mathrm{L}$. Therefore, the $5.2 \mathrm{~kg}$ release is equivalent to $4.37 \mathrm{~L}$ total, of which $5.3 \%$ or $0.23 \mathrm{~L}$ are solid, and $4.14 \mathrm{~L}$ are liquid. Note that exact volume fractions and densities could be derived for conversions above, but the amount of solids entrained varies with each bump, so a somewhat conservative approach was taken.

The overall $Q^{*}$ ULD for waste is found by weighting Table 8-2 values by the volume released,

$$
\begin{array}{ll}
\text { On-Site } & Q^{*} \mathrm{ULD}=\left(1.07 \times 10^{5}\right)(0.23)+(797)(4.14)=27,900 \mathrm{~Sv} \\
\text { Off-Site } & Q^{*} \mathrm{ULD}=\left(1.84 \times 10^{5}\right)(0.23)+(845)(4.14)=45,800 \mathrm{~Sv}
\end{array}
$$

Therefore, total on-site worker dose is

$$
\text { On-Site } \quad \mathrm{D}=(27,900 \mathrm{~Sv})\left(5.58 \times 10^{-3} \mathrm{~s} / \mathrm{m}^{3}\right)\left(3.33 \times 10^{-4} \mathrm{~m}^{3} / \mathrm{s}\right)=0.05 \mathrm{~Sv}
$$

and off-site receiver dose is:

$$
\text { Off-Site } \quad D=(45,800)\left(8 \times 10^{-6} \mathrm{~s}^{3} \mathrm{~m}^{3}\right)\left(3.33 \times 10^{-4} \mathrm{~m}^{3} / \mathrm{s}\right)=1.2 \times 10^{-4} \mathrm{~Sv}
$$

Toxic chemical consequences are found using the methods of WHC-SD-WM-SARR-011 (1996). Table 3-8 of the reference provides the toxic limit sum-of-fractions for toxic chemical dose relative to allowed thresholds. Pertinent sum-of-fraction data from Table 3-8 of the reference are summarized in Table 8-3. Values are given for continuous release consistent with the radiological evaluation. When viewed as a continuous release, values in the table are applied to the released volumes of $0.23 \mathrm{~L}$ solid and $4.14 \mathrm{~L}$ liquid over a duration of 21,600 seconds. A puff evaluation is made for a single worst release which is $1 / 10$ the total, with a duration of 60 seconds per Cowley et al. (2000). This means the puff release result is a factor of $1 / 10 * 21,600 / 60=36$ times larger than the continuous release result. The puff evaluation is made because the release history for the tank bump accident presented earlier has aspects of both 
Table 8-3. Sum-of-Fraction of Risk Guidelines for a Unit Release of Chemicals (WHC-SD-WM-SARR-011 1996).

\begin{tabular}{|l|c|c|c|}
\hline \multicolumn{4}{|c|}{ Continuous Release, units s/L } \\
\hline \multirow{2}{*}{ Waste Type } & Receptor Location & \multicolumn{2}{|c|}{ Accident Frequency 1/yr. } \\
\cline { 3 - 4 } & & $\mathbf{1 0}^{-2}$ to $\mathbf{1 0}^{-4}$ & $\mathbf{1 0}^{-4}$ to 10 \\
\hline DST Liquids & On-Site & 750 & 210 \\
DST Solids & On-Site & 3300 & 630 \\
DST Liquids & Off-Site & 8.4 & 0.62 \\
DST Solids & Off-Site & 15 & 2.8 \\
\hline
\end{tabular}

a continuous and a puff release, and because toxic chemical consequences are sensitive to peak concentration. Also, separate evaluations are made for frequency bins of $10^{-4} / \mathrm{yr}$ to $10^{-6} / \mathrm{yr}$, corresponding to current waste temperatures, and level from $10^{-2} / \mathrm{yr}$ to $10^{-4} / \mathrm{yr}$, corresponding to operation at LCO temperatures, given results from Section 6 above.

A release duration of $21,600 \mathrm{~s}$ and volumes of $0.23 \mathrm{~L}$ solid and $4.14 \mathrm{~L}$ liquid are applied for the continuous release. The last bump event produces the largest puff which accounts for about $10 \%$ of the total release, hence, volumes of $0.023 \mathrm{~L}$ solid and 0.414 liquid are applied. The result of the evaluation is given in Table 8-4. Clearly, on-site puff release consequences are not within guidelines, while all other calculated consequences are within guidelines.

Table 8-4. Toxic Consequence Evaluation Results.

\begin{tabular}{|c|c|c|}
\hline \multirow[b]{2}{*}{ Receptor Location } & \multicolumn{2}{|c|}{ Accident Frequency $1 / y r$. } \\
\hline & $10^{-2}$ to $10^{-4}$ & $10^{-4}$ to $10^{-6}$ \\
\hline \multicolumn{3}{|c|}{ Continuous Release } \\
\hline On-Site & 0.18 & 0.047 \\
\hline Off-Site & 0.0018 & 0.00015 \\
\hline \multicolumn{3}{|c|}{ Puff Release } \\
\hline On-Site & 6.5 & 1.7 \\
\hline Off-Site & 0.07 & 0.005 \\
\hline
\end{tabular}

Continuous release results are within guidelines, while puff release results are above guidelines. 
RPP-6213 REV 0

This page intentionally left blank.

FAI/00-14, Rev. 0 


\subsection{SUMMARY AND DISCUSSION OF ASSUMPTIONS AND APPROXIMATIONS}

\subsection{TANK BUMP PHYSICAL MODELS}

The steam bump model is subject to a number of simplifying, realistic, and conservative assumptions. The important assumptions are listed below together with their estimated degree of conservatism.

A1.1 Bubble growth is limited by steam transport within the bubble.

Degree of Conservatism: This assumption is believed to be moderately conservative in that the resistance to growth imposed by the thermal boundary layer on the liquid side of the bubble surface is at least as important as the diffusional resistance to growth on the vapor side.

A1.2 The flow field within the bubble is approximated by a Hill's vortex for the purpose of estimating the resistance to steam mass transfer within the bubble.

Degree of Conservatism: This assumption is regarded as realistic since it has been supported by bubble growth data available in the literature.

A1.3 The rise velocity of the buoyant parcel is estimated by appealing to an available correlation for the rise velocity of gas-bubble ensembles.

Degree of Conservatism: This assumption is realistic as the quasi-steady application of the correlation to growing, but essentially inertialess bubbles is valid.

A1.4 The mixing of the rising buoyant parcel with the surrounding supernatant is well described by the Morton-Taylor-Turner entrainment equation.

Degree of Conservatism: This very realistic assumption is supported by numerous experiments on mixing of jets, plumes, or buoyant parcels with the ambient fluid.

A1.5 The mass of aerosol produced by steam bubble breakthrough is estimated with the Kataoka-Ishii correlation.

Degree of Conservatism: The use of this correlation is regarded as realistic since it is consistent with the available data in the high gas-flow regime.

A1.6 The Meyer et al. (1997) semi-empirical expressions are used to assess the total void volume and the total mass of nonconvective material that are assumed to suddenly appear and participate in the buoyant displacement. 
Degree of Conservatism: This assumption is probably very conservative for several reasons. The evidence from the six DSTs that exhibit buoyant displacements suggests that the buoyant release occurs gradually over time $(\sim 1 \mathrm{~min})$ as opposed to the sudden release of the single, large parcel assumed in the model. The tanks believed to be most susceptible to a bump and chosen for tank bump model evaluation are sludge tanks. It is doubtful that neutral buoyancy can be attained in the non-convective layers in these tanks through buildup of noncondensible gases. The Meyer et al. (1997) energy criterion for gas release indicates that noncondensable gas generation cannot bring the non-convective layers to a buoyant condition, even at their boiling points. Also, there are some reasons to believe that gas-solid or steam-solid attachment is difficult in sludgelike materials so that gas or vapor retention is limited in these materials (Kovach 2000).

\subsection{TANK BUMP FREQUENCY CALCULATION}

For the scenario frequency quantification in Section 7.0, underlying assumptions are revisited and discussed here.

A2.1 Initiating events for ventilation failure are random failures, lightning strikes, loss of off-site power, and seismic events.

Degree of Conservatism: Excluding other potential initiators is non-conservative, strictly speaking, but not important as a practical matter. Other initiating events can be postulated, such as fires, vehicular accidents, extreme weather, sabotage, etc. The frequency analysis is limited in scope and none of the additional initiators mentioned here has the potential to alter the conclusions of Section 7.0. Assuming repair activities are unaffected by the initiating event, any postulated initiator can be compared to the initiating event frequency created by random failures, which has an expected value of 0.03 per year, to gauge its importance. (Note that LOSP and seismic events were given explicit consideration precisely because repair activities are not independent of the initiator.) To significantly alter the conclusions in Section 7.0 then, a new, additional postulated initiating event should have a frequency of 0.1 per year, assuming repairs are independent of the initiator. Such an event is hard to postulate.

A2.2 Repairing the ventilation system is the only way to prevent a tank bump.

Degree of Conservatism: This assumption is consistent with the DST and AWF Tank Waste Temperature Controls. For the long recovery periods available for tank bump, it is conservative because other means of reducing waste temperature can be considered: portable systems, water addition, etc. For the current conditions, this assumption proves to be unimportant because the frequency is readily shown to beyond extremely unlikely with the exception of the large seismic events.

A2.3 Recovery times can be estimated from the WFD RAM. 
Degree of Conservatism: This assumption is necessitated in part by the limited scope of the current work. The WFD RAM serves as the best source of data of restoration times and corrective maintenance times. The WFD corrective maintenance times were described as "generic" in the RAM documents, which means that applying them to the safe storage scenario is reasonable in the absence of better values.

A2.4 Corrective maintenance activities require tank farm radiological area access, but neither pit access nor non-radiological area access.

Degree of Conservatism: This is a best-estimate assumption based on the location and configuration of the ventilation system. Pit access would be relevant for replacing waste contacted components; neglecting large seismic events, the likelihood that this would be necessary to repair the ventilation system is judged to be negligible. On the other hand, repairing the ventilation system without radiological access is not likely neither.

A2.5 Ventilation system failure rate can be estimated from the 1997 availability data, which uses the downtime for the exhaustor fans as a basis.

Degree of Conservatism: This assumption is best-estimate in the sense that the success criteria is the primary ventilation system alone can cool the waste sufficiently to prevent a bump; the annular ventilation system and ancillary components, such as condensers, are not needed. A simplifying assumption used to calculate the ventilation system failure rate is that down time due to preventive maintenance is ignored and treated as corrective maintenance. Uncertainties are treated by using random variables for the availability and mean repair times.

\section{A2.6 The frequency of corrective maintenance activities from the WFD RAM can be applied to safe storage scenarios.}

Degree of Conservatism: This is a best-estimate assumption in the absence of information specific to the safe storage scenario. The corrective maintenance activities defined for the WFD RAM are said to be generic. The relative frequencies are in agreement with the observed high availability of the ventilation system: i.e., (1) the vast majority of ventilation system losses can be recovered by simple restoration activities that take only a few hours, and (2) simple corrective maintenance functions are much more frequent than long duration ones.

Uncertainties are treated by using random variables for the relative frequencies of the various activities.

A2.7 Failure rates and recovery times for the current operating conditions can be applied to the hypothetical conditions of no ventilation controls.

Degree of Conservatism: If no ventilation controls are assumed, the operator uses the ventilation system intermittently to keep waste temperatures below LCO. For this hypothetical scenario, the availability, failure rates, etc., cannot be known. For the sake of comparison, the values derived the current conditions are retained. 
A2.8 For small seismic events, failure to repair probability is based on the hypothetical no ventilation controls case, which has a relatively small time available for recovery.

Degree of Conservatism: When made a priori, this is a conservative assumption. Results are insensitive to this value, however, because the large events dominate the failure frequency calculation.

A2.9 For large seismic events, failure to repair probability approaches one at $0.8 \mathrm{~g}$ or above.

Degree of Conservatism: This is conservative in that there is some probability that a tank can withstand more than $0.8 \mathrm{~g}$ without compromising its function. This assumption is academic, however, because tank bumps prevent few hazards in relation to the initiating event.

\subsection{TANK BUMP CONSEQUENCE EVALUATION}

\section{A3.1 Tank 241-AZ-102 is chosen for a consequence evaluation.}

Degree of Conservatism: As shown in Section 4.2, and found by application of criterion 6 of Section 6.1 , there is insufficient noncondensible gas generation in this tank to cause a buoyant displacement given available information at this time. Therefore, this is an example calculation for a tank that satisfies thermal criterion for steam bump potential. It is conservative to choose tank 241-AZ-102 over tank 241-AY-102 based on application of steam generation in equation (4-16), but realistic to make this choice given time to heat the waste to saturation conditions.

A3.2 Initially released gas volume and time between bumps are found using the Meyer et al. (1997) expressions.

Degree of Conservatism: These are best-estimate models for tanks observed to undergo buoyant displacements today, but per (A1.6) and (A3.1), this is conservative for application to tank 241-AZ-102.

A3.3 Leakage paths from the tank headspace are gaps around the 42 " riser shield plugs, and inlet/outlet HEPA filters are assumed not present.

Degree of Conservatism: Leakage path values follow the Barton and Bingham (1999) methodology, and are best-estimate, although it is unclear whether or not other leakage paths may exist for any given tank. Neglect of HEPA filters is slightly conservative because the headspace pressure attained during a bump is at the threshold for failure, and this is a simplifying assumption. The actual effect of filters would be difficult to justify.

A3.4 Aerosol retention in sluice and pump pits is not credited. 
Degree of Conservatism: This is somewhat conservative, but most aerosols are released via the normal inlet and outlet paths, so the contribution to release from the pits is small.

A3.5 Approximate solid fractions are used to convert released mass to solid and liquid volumes.

Degree of Conservatism: The mass of solids entrained per bump varies throughout the sequence of bumps, so a somewhat conservative approach was taken, whose impact is deemed small.

A3.6 On-site and off-site doses assume an 8-hour release duration.

Degree of Conservatism: $90 \%$ of the waste is released over 6 hours (12 bumps at 30 minute intervals), so the worker shift assumption is reasonable, and the off-site conservatism is small.

A3.7 The effect of further bumps after settling of waste from the original sequence of bumps is not quantified.

Degree of Conservatism: Further sets of bumps would result in the same sources as predicted for the original sequence described here, and this is mentioned in Section 8.3. However, considerable time would elapse for sufficient noncondensible gas generation and accumulation to trigger a new set of bumps. Even though not considering further sets of bumps is non-conservative, this is judged to be an unlikely event following an originally unlikely event. 


\section{RPP-6213 REV 0}

This page intentionally left blank. 


\section{RPP-6213 REV 0}

\subsection{REFERENCES}

Alstad, A. T., 1993, Riser Configuration Document for Single-Shell Waste Tanks, WHC-SD-RE-TI-053, Rev. 8, Westinghouse Hanford Co., Richmond, WA.

Barton, W. B., and Bingham, J. D., 1999, Gas Release Event Safety Analysis Tool Pedigree Database for Hanford Tanks, HNF-SD-WM-TI-806, Rev. 2-A, Lockheed Martin Hanford Corp., Richland, WA.

Bendixsen, R. B., 1989, History of Tank Bumps in Aging Waste Tanks, Letter Report to D. A. Clapp.

Briggs, G. A., 1969, Plume Rise, U.S. Atomic Energy Commission Critical Review Series, TID-25075, NTIS.

Brown, W. G., 1962, Natural Convection Through Rectangular Openings in Partitions-2. Horizontal Partitions, Int'l. J. Heat Mass Transfer, Vol. 5, pp. 869-878.

Brownell, L. E., 1958, Instability of Steel Bottoms in Waste Storage Tanks, WHC-SD-WM-TI-406, Rev. 0, Doc. No. HW-57274, General Electric, Hanford Atomic Products Operation, Richland, WA.

Buck, F. G., 1993, Potential Lightning Strike Events, PNL-9800, Pacific Northwest Laboratory, Richland, WA.

Carlson, A. B., 1999, Waste Feed Delivery Technical Basis, Volume IV: Waste Feed Delivery Operations and Maintenance Concept, HNF-1939-Vol. IV, Rev. 0, Numatec Hanford Corporation, Richland, WA.

Carlson, A. B., 1999a, Waste Feed Delivery System Phase 1 Preliminary Reliability, Availability, Maintainability Analysis, HNF-2863, Rev. 1, Numatec Hanford Corporation, Richland, WA.

Childs, K. W., 1991, Heating 7.1 User's Manual, K/CSD/TM-96, Draft, Martin Marietta, Oak Ridge, TN.

Cowley, W. L., and Stepnewski, D. D., 1994, Evaluation of Hazards from Lightning Strikes to Tank Farm Facilities, WHC-SD-WM-SARR-027, Rev. 0, Westinghouse Hanford Co., Richland, WA.

Cowley, W. L., Sandgren, K. R., and VanKeuren, J. C., 2000, Radiological Source Terms for Tank Farm Safety Analysis, RPP-5294, Rev. 0, CH2M HILL Hanford Group, Inc., Richland, Washington.

Crea, B. A., Sathyanarayana, K., and Ogden, D., 2000, Parametric Analyses of Heat Removal from High-Level Waste Tanks, RPP-5637, Rev. 0, CH2M HILL Hanford Group, Inc., Richland, WA. 
DOE, 1994, Natural Phenomena Hazards Design and Evaluation Criteria for Department of Energy Facilities, DOE-STD-1020, U.S. Dept. of Energy, Washington, DC.

Epstein, M., 1988, Surface Evaporation Model, FAI Internal Memorandum.

Epstein, M., and Burelbach, J. P., 1998, Experimental and Theoretical Turbulent Diffusion Modeling of Light Gas Releases in a Tank Headsepace: 2. Local Releases, FAI/98-50, Fauske \& Associates, Inc., Burr Ridge, IL.

Epstein, M., and Burelbach, J. P., 2000a, Transient Vertical Mixing by Natural Convection in a Wide Mixing Layer, Int'l. J. Heat and Mass Transfer, Vol. 43, pp. 321-325.

Epstein, M., and Burelbach, J. P., 2000b, Vertical Mixing Above a Steady Circular Source of Buoyancy, Int'l. J. Heat and Mass Transfer (in press).

Epstein, M., Fauske, H. K., and Plys, M. G., 1999, Hanford Tank 24I-C-106 Temperature Limit \& Steam Bump Evaluation, FAI/99-18, Fauske \& Associates, Inc., Burr Ridge, IL.

FAI, 1989, Independent Review of Aging Waste Tank "Bump" Phenomena, FAI/89-94, Fauske \& Associates, Inc., Burr Ridge, IL.

Garner, F. H., Ellis, S. R. M., and Lacey, J. A., 1954, The Size Distribution and Entrainment of Droplets, Trans. Instr. Chem. Engrs., Vol. 32, pp. 222-324.

Gauglitz, P. A., Rassat, S. D., Bredt, P. R., Konynenbelt, J. H., Tingey, S. M., and Mendoza, D. P., 1996, Mechanisms of Gas Bubble Retention and Release: Results for Hanford Waste Tanks 241-S-102 and 24I-SY-103 and Single-Shell Tank Simulants, Pacific Northwest National Laboratory Report PNNL-11298, Richland, WA.

Ginsberg, T., 1983, Aerosol Generation from Sparging of Molten Pools of Corium by Gases Released During Core-Concrete Interactions, Proc. Int'l Mtg. Light Water Reactor Severe Accident Evaluation, Vol. 2, Cambridge, MA.

Golub, S. I., 1970, Investigation of Moisture Carryover and Separation in Evaporation Apparatus, Candidates Dissertation, MEI (quoted from Kataoka and Ishii [1984]).

H-2-68304, Rev. 4, Structural Concrete Pump Pit Plans - Sections - Details Tank 241-AZ-102 $\& 101$.

H-2-68305, Rev. 4, Structural Concrete Sluice Pit Plans - Sections - Details Tank 241-AZ-102 $\& 101$.

H-2-68423, Rev. 2, Tank Riser and Airlift Circulator Details.

H-Unknown-1, Air Lift Circulator and Riser Extension Details, Bldg. 241-AZ.

Han, F. C., 1996a, Structural Integrity and Potential Failure Modes of the Hanford High-Level Waste Tanks, WHC-SD-TWR-RPT-002, Rev. 0, Westinghouse Hanford Co., Richland, WA. 
Han, F. C., 1996b, DELPHI Expert Panel Evaluation of Hanford High Level Waste Tank Failure Modes and Release Quantities, WHC-SD-TWR-RPT-003, Rev. 0, Westinghouse Hanford Co., Richland, WA.

Hanson, G. L., 1955, 241-SX-Operation, Internal Letter, General Electric Co.

Harmon, M. K., 1958, Radiation Occurrence, Form found within WHC-SD-WM-TI-106, Rev. 0.

HNF-SD-WM-SAR-067, Rev. 1, 2000, Tank Waste Remediation System Final Safety Analysis Report, Rev. 1-H, CH2M HILL Hanford Group, Inc., Richland, Washington.

Hu, T. A., 1999, Empirical Rate Equation Model and Rate Calculations of Hydrogen Generation for Hanford Tank Waste, HNF-3851, Rev. 0, Lockheed Martin Hanford Corp., Richland, WA.

Hu, T. A., Barker, S. A., Bingham, J. D., and Kufahl, M. A., 2000, Steady State Flammable Gas Release Rate Calculation and Lower Flammability Level Evaluation for Hanford Tank Waste, RPP-5926, Rev. 0, CH2M HILL Hanford Group, Inc., Richland, WA.

Hunt, N., 2000, Presentation at the DOE Regulatory Unit Topical Meeting with BNFL on Sellafield Database/Risk Objectives, Richland, WA.

Jo, J., 1990, The History and Existing Evaluations of the Tank Bump, WHC-SD-WM-TI-406.

Jones, B. L., 1988, Aging Waste Tank Bump Sensitivity to Thermal Conductivity and Heat Capacity, Incorporating the Assumption of N-Reactor Shutdown, Internal Memo to L. A. Mihalik, Westinghouse Hanford Co., found within WHC-SD-WM-TI-406, Rev. 0.

Kataoka, I., and Ishii, M., 1984, Mechanistic Modeling of Pool Entrainment Phenomenon, Int'l J. Heat Mass Transfer, Vol. 27, pp. 1999-2014.

Katsaros, K. B., Liu, W. T., Businger, J. A., and Tillman, J. E., 1977, Heat Transport and Thermal Structure in the Interfacial Boundary Layer Measured in an Open Tank of Water in Turbulent Free Convection, J. Fluid Mech., Vol. 83, pp. 311-355.

Kovach, J. L., 2000, Comments on Gas Solid Attachment in Liquid Phase, Memorandum to SY-101 SubTAP.

Kuhn, W. L., 1988, Independent Review of Aging Waste Tank "Bump" Analyses, Final letter report from Pacific Northwest Laboratory, Richland, WA, found within WHC-SD-WM-TI-406, Rev. 0.

Kummerer, M., 1994, Topical Report on Heat Removal Characteristics of Waste Storage Tanks, WHC-SD-WM-SARR-010, Rev. 0, Westinghouse Hanford Co., Richland, WA.

Malinovic, B., Plys, M. G., and Epstein, M., 2000, Hanford Waste Tank Source Term Model HADCRT 1.1: User's Manual, FAI/00-3, Fauske \& Associates, Inc., Burr Ridge, IL. 


\section{RPP-6213 REV 0}

Marble, W. J., 1983, Preliminary Report on the Fission Product Scrubbing Program, General Electric Report, NEDO-30017.

Meyer, P. A., Brewster, M. E., Bryan, S. A., Chen, G., Pederson, L. R., Stewart, C. W., and Terrones, G., 1997, Gas Retention and Release Behavior in Hanford Double-Shell Waste Tanks, PNNL-11536, Rev. 1, Pacific Northwest National Laboratory, Richland, WA.

Meyer, P., and Wells, B., 2000, Understanding Gas Release Events in Hanford Double Shell Tanks, in WM'00 Proceeding of the Symposium on Waste Management, Tucson, Arizona.

Moody, F. J., 1986, Dynamic and Thermal Behavior of Hot Gas Bubbles Discharged into Water, Nuclear Engng. \& Design, Vol. 95, pp. 47-54.

Morton, B. R., Taylor, G. I., and Turner, J. S., 1956, Turbulent Gravitational Convection From Maintained and Instantaneous Sources, Proc. R. Soc. Lond., Vol. A234, pp. 1-23.

Perry, J. H., 1950, Chemical Engineers' Handbook, 3rd Edition, McGraw-Hill, New York, NY, p. 546.

Reid, R. C., and Sherwood, T. K., 1966, The Properties of Gases and Liquid, Their Estimation and Correlation, 2nd ed., McGraw-Hill, New York.

Ruckenstein, E., Dang, V. D., and Gill, W. H., 1971, Mass Transfer with Chemical Reaction from Spherical One- or Two-Component Bubbles or Drops, Chem. Eng. Sci., Vol. 26, pp. 647-659.

Sathyanarayana, K., 1996, Evaluation of Potential and Consequences of Steam Bump in High Heat Waste Tanks... Validation of GOTH Computer Code, WHC-SD-WM-CN-022, Rev. 0, Westinghouse Hanford Co., Richland, WA.

Shultz, M. V., Jr., 1994, Analysis of Power Loss Data for the 200 Area Tank Farms in Support of $K$ Basin SAR Work, WHC-EP-0811, Westinghouse Hanford Co., Richland, WA.

Slezak, S. E., Williams, D. C., Cheng, W., Gelbard, F., and Bratzel, D. R., 1998, Refined Safety Analysis Methodology for Flammable Gas Risk Assessment in the Hanford Site Tanks, Sandia National Laboratory, HNF-SD-WM-ES-410, Rev. 2, DE\&S Hanford, Richland, WA.

Stewart, C. W., Brewster, M. E., Gauglitz, P. A., Mahoney, L. A., Meyer, P. A., Recknagle, K. P., and Reid, H. C., 1996, Gas Retention and Release Behavior in Hanford Single-Shell Waste Tanks, PNNL-11391, Pacific Northwest National Laboratory, Richland, WA.

Stewart, C. W., 2000, personal communication.

Tomlinson, R. E., 1955, Storage of High Activity Wastes, WHC-SD-WM-TI-406, Rev. 0, Westinghouse Hanford Co., Richland, WA. 
Turner, J. S., 1973, Buoyancy Effects in Fluids, Cambridge University Press, Cambridge.

Waters, E. D., Allen, G. K., and Place, D. E., 1991, Analysis of Tank Bump Potential During InTank Washing Operations Proposed for the 241-AZ Tanks, WHC-SD-WM-ER-114, Rev. 0, Westinghouse Hanford Co., Richland, WA.

Wesley, D. A., and Nakiki, S. D., 1992, A Safety Assessment for Evaluating the Efficacy of Pump Mixing to Mitigate Episonic Gas Release Tank 241-101-SY: Hanford Site, Richland, Washington, Draft.

WHC-SD-WM-SARR-011, 1996, Toxic Chemical Considerations for Tank Farm Releases, Rev. 2., Westinghouse Hanford Co., Richland, WA.

Willingham, C. E., 1994, Thermophysical Properties of Hanford High-Level Tank Wastes - A Preliminary Survey of Recent Data, PNL-9419, Pacific Northwest Laboratory, Richland, WA.

Zach, J. J., 1996, Probability, Consequences, and Mitigation for Lightning Strikes to Hanford Site High-Level Waste Tanks, WHC-SD-WM-ES-387, Rev. 1, Westinghouse Hanford Co., Richland, WA. 
RPP-6213 REV 0

This page intentionally left blank. 


\section{A.0 TANK WASTE CHARACTERIZATION DATA}

\section{A.1 E-MAIL CORRESPONDENCE}

From: Blaine_A_Crea@RL.gov

Sent: Tuesday, April 04, 2000 6:38 PM

To: plyserauske.com

Cc: Thomas_G_Tom_Goetz@apimc01.rl.gov; David_R_Bratzeldapimc01.rl.gov

Subject: RE: ${ }^{-D S T}$ Inlet Information Needs

The following explanation of the ventilation systems in the aging waste facility hopefully will clarify item 8 for you: I'll dig up appropriate reference material for you later.

There are four tanks in this facility. Two in AY tank farm (AY-101 and $A Y-102)$. Two in AZ tank farm (AZ-101 and AZ-102)

First each tank has both a primary (Dome space) and secondary (Annulus) ventilation system.

I'Il discuss the annulus vent systems first. They are in general capable of drawing about 1000 CFM of air through the tank annulus when they're operating this is true for the current configuration of all the systems.

Each tank in the AY tank farm has a similar but totally separate annulus vent system. The two tanks in the AZ farm, however, share a common exhauster i.e. fans filters, stack, monitors.

All four tanks share elements of a common primary vent system. Each tank has, however, a separate recirculation cooling system. The included simplified sketch of the primary system may help the show the component arrangement.

The components in the lower right corner of the sketch are housed in a separate building outside of the farms. This provides tank vacuum and discharge cleanup. It also can provide some cooling, but the original design basis for the chiller driven condenser was to cool the off gas to slightly above $O$ centigrade and so remove almost all the moisture and the associated tritium. It also is service by dedicated UPS's and a backup diesel generator with an automatic start/transfer switch.

The components of the recirculation modules shown next to each tank are designed to remove most of the heat over the long run. There is one of these recirculation modules with associated condensers, fans, cooling towers, coolant loops etc for each tank. 


\section{RPP-6213 REV 0}

From: Sathyanarayana, K

Sent: Wednesday, April 05, 2000 4:36 PM

To: 'plysefauske.com,'; Bratzel, David R; Crea, Blaine A

Cc: Sathyanarayana, $\mathrm{K}$

Subject: Pumps and Sluice Pits Information

Marty and Dave,

Blaine was looking for information on details of Pump and sluice Pits for Tank 241-A2-101. I donot have drawings but I could find the following information in old SAR document (SD-HS-SAR-010, Rev.1).

Above each of the 241-Az tanks is a Pump Pit, located in the center of the tank, and two sluice Pits located opposite to each other. The Pump Pits have approximate inside dimensions of $8 \mathrm{ft}$ by $12 \mathrm{ft}$ by 7 ft deep with 2.5 ft thick concrete cover blocks. The walls are 1 ft thick concrete. The Sluice Pits have approximate inside dimensions of $6 \mathrm{ft}$ by 8 ft by $7 \mathrm{ft}$ deep with $2 \mathrm{ft}$ thick concrete cover blocks. The walls are 1 ft thick concrete.

The pump and sluice pit covers are purposely not air tight to permit some air leakage into the tank. The pits have drains and base plate openings for a pump or sluice nozzle, going directly into the vapor space of the tanks. Air can leak in through the cracks between cover blocks and the penetrations in the blocks ( $1 . e .$, holes for mechanisms to operate the sluice nozzle, valves, etc.).

I hope this information is correct and represen present conditions. Hope this is the information that you are looking for and to make sure Blaine can verify this.

Thanks

Sathya

From: Blaine_A_CrealRL.gov

Sent: Monday, April 10, $20009: 44$ AM

To: plysefauske.com

CC: Thomas G_Tom_Goetz@APIMC01.RL.GOV; David_R_Bratzel@APIMC01.RL.GOV

Subject: $\mathrm{RE}$ : ${ }^{-D S T}$ Inlet Information Needs

The only information that I have been able to come up with on filter failure threshold is from ASME AGA-1. This is the standard that all HEPA filters must meet.

The performance test requires that they withstand a differential pressure of 10 inches of water while subjected to an air stream that contains 1 lbm water droplets $/ 1000$ cubic feet of air for an hour. The airflow rate during this test is adjusted to maintain the 10 inches of water differential pressure throughout the test.

The filter is a $500 \mathrm{SCEM}$ rated filter. That means that it has an initial (clean) pressure drop of 1 inch of water at 500 SCFM. Since the pressurization of the dome will be a back flow it would seem reasonable that this would be the filter characteristic at the initiation of the backflow sequence. 
RPP-6213 REV 0

From: K Sathyanarayana@RL.gov

Sent: Monday, April 10, $200012: 32$ PM

To: plyserauske.com

Cc: David_R_BratzelCapimc01.rl.gov; Blaine_A_Crea@apimc01.rl.gov;

K_Sathyanarāyanaeapime01.rl.gov

subject: RE: Pumps and Sluice Pits Information

Marty-

The primary Tank Ventilation System contains individual controlled air inlets and a common off-gas exhaust for the four $A Y$ and $A Z$ Tank Farm waste tanks. The individual tank air inlet consists of a heater, pre-filter, high-efficiency particulate air (HEPA) filter, flow control valve and a vaccum relief valve.

Inlet Equipment: Heater, Pre-filter and HEPA filter

1. The ouside air inlet has an electric 3-phase heater to protect the filters from excessive humidity or frost. Heaters are sized for an air flow of 100 to $500 \mathrm{cfm}$.

2. The pre-filter protects the HEPA filter from unnecessary particulate loading which would shorten its life. The pre-filter efficiency is about 25 to 308 per ASHRAE standard 52-68. It is 12" by 24" by 2", UL Class 1, fiber glass media with nonflammable frame. The filter surface area is atleast 8.5 ft2.

3. The HEPA filter is necessary to protect the environment against potential backflow from the waste tank inlet. The filter is nuclear grade, 12" by 24" by 11 1/2" with a nominal rating of $500 \mathrm{cfm}$. The operating pressure drop of 3" of water for the nominal flow rate. The maximum pressure for leak is $10 "$ of wate gauge (I need to get confirmation of this value).

The inlet pipe is 6 inches diameter schedule 40.

Thanks

Sathya 
RPP-6213 REV 0

\section{A.2 DRAWINGS}


RPP-6213 REV 0

Figure A-1. System Schematic Diagram.

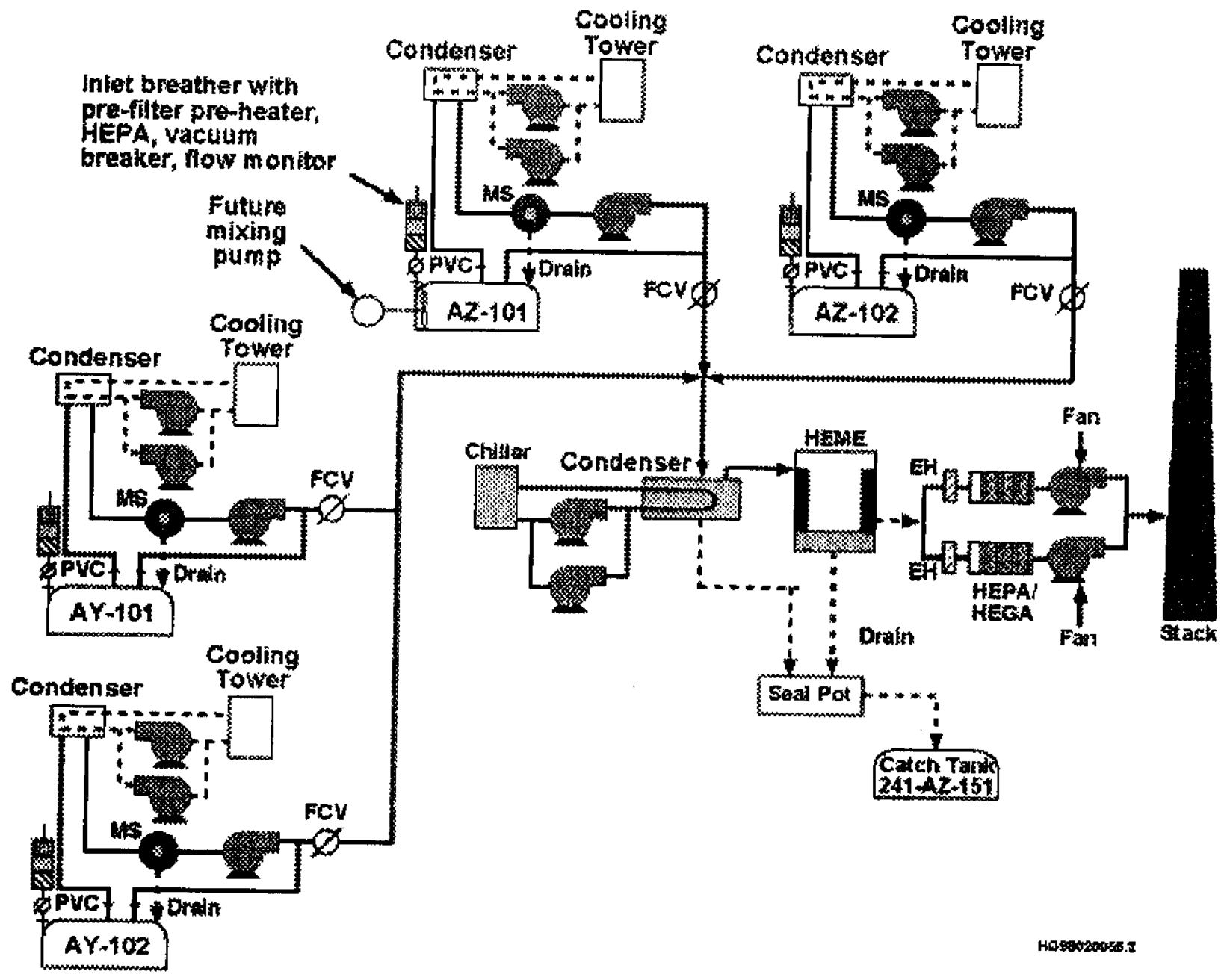


Figure A-2. Structural Concrete Sluicing Pit Plans - Part 1 of 2.

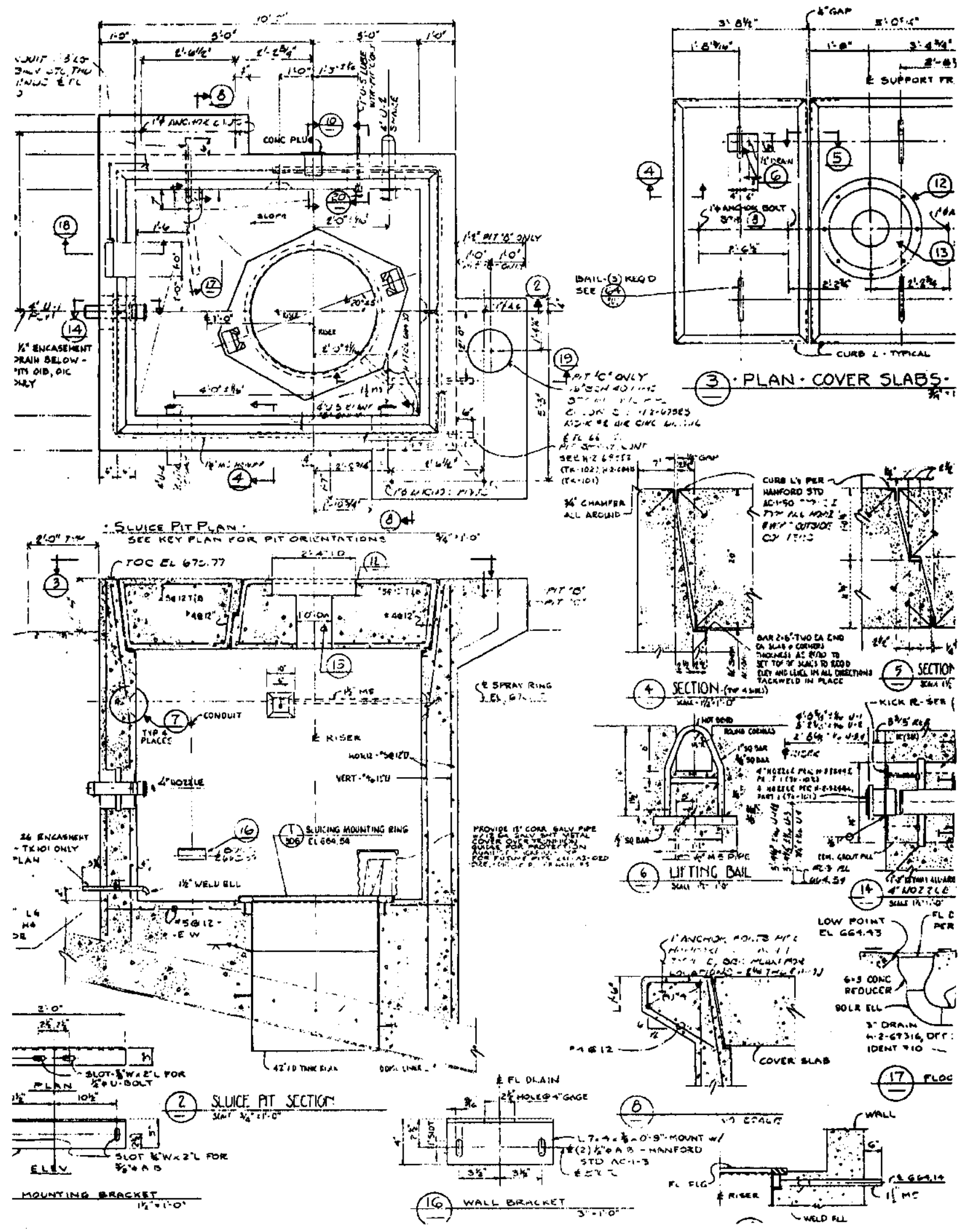

FAI/00-14, Rev. 0 
Figure A-3. Structural Concrete Sluicing Pit Plans - Part 2 of 2.
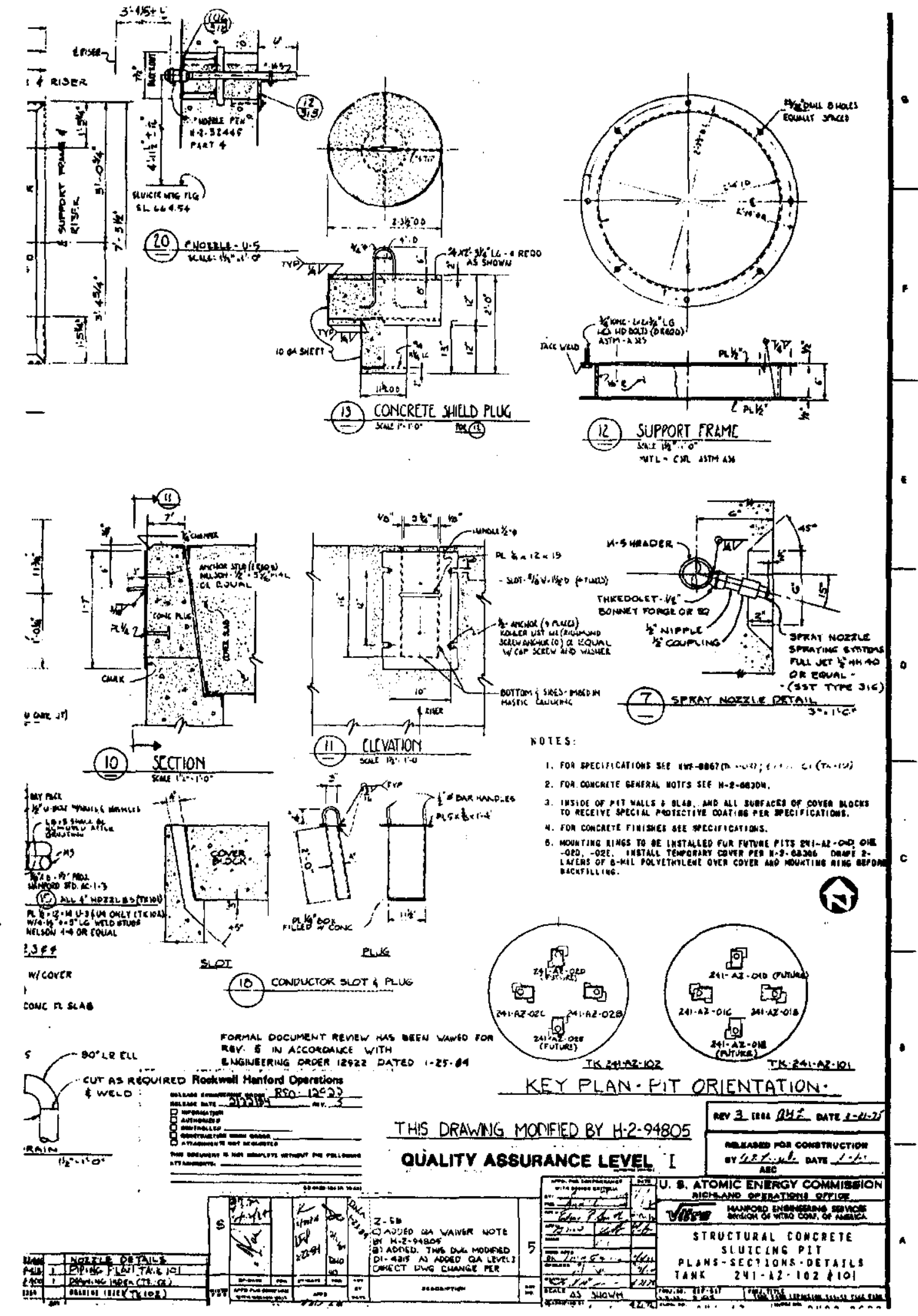

FAI/00-14, Rev. 0 
Figure A-4. Structural Concrete Pump Pit Plans - Part 1 of 2.

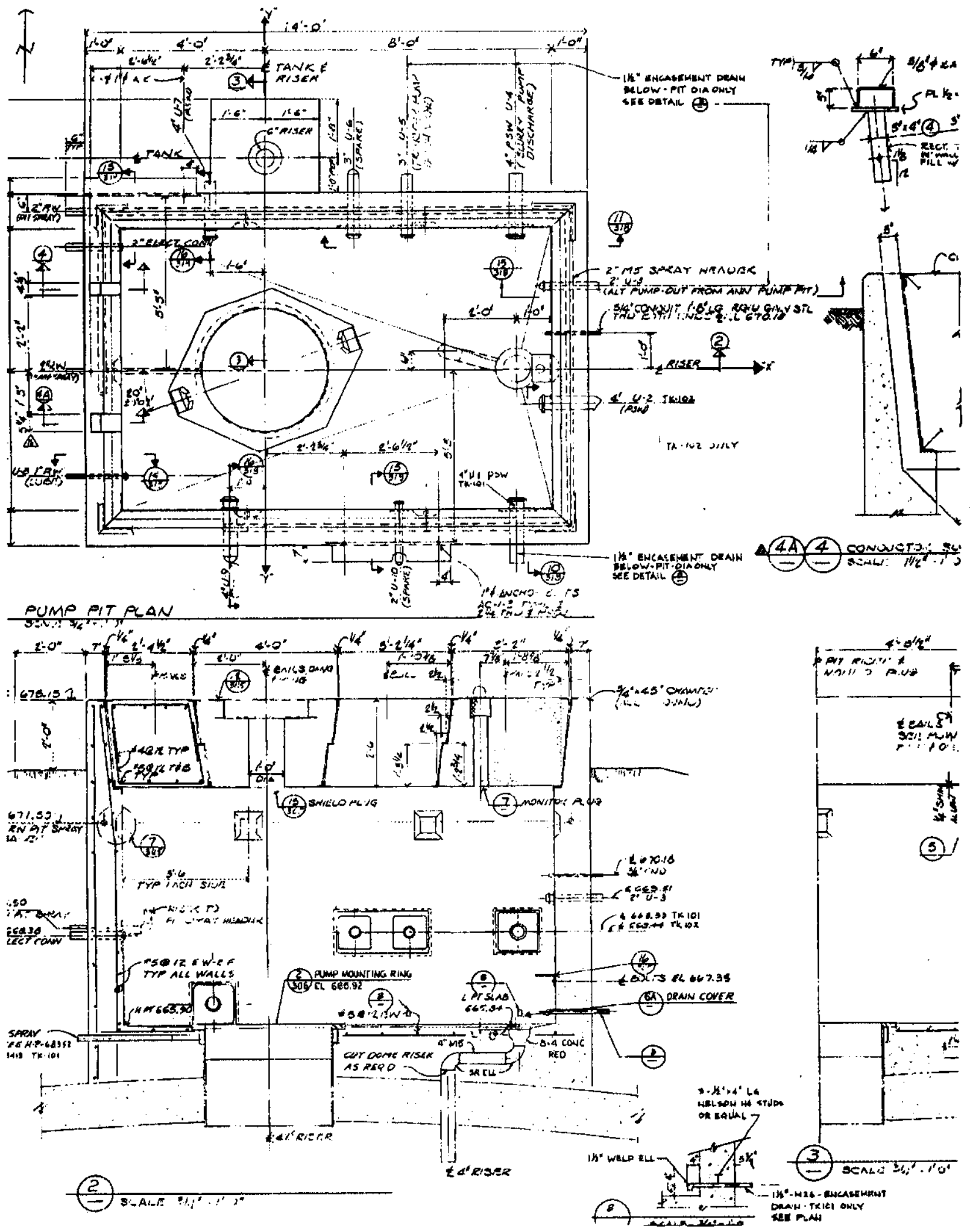


Figure A-5. Structural Concrete Pump Pit Plans - Part 2 of 2.

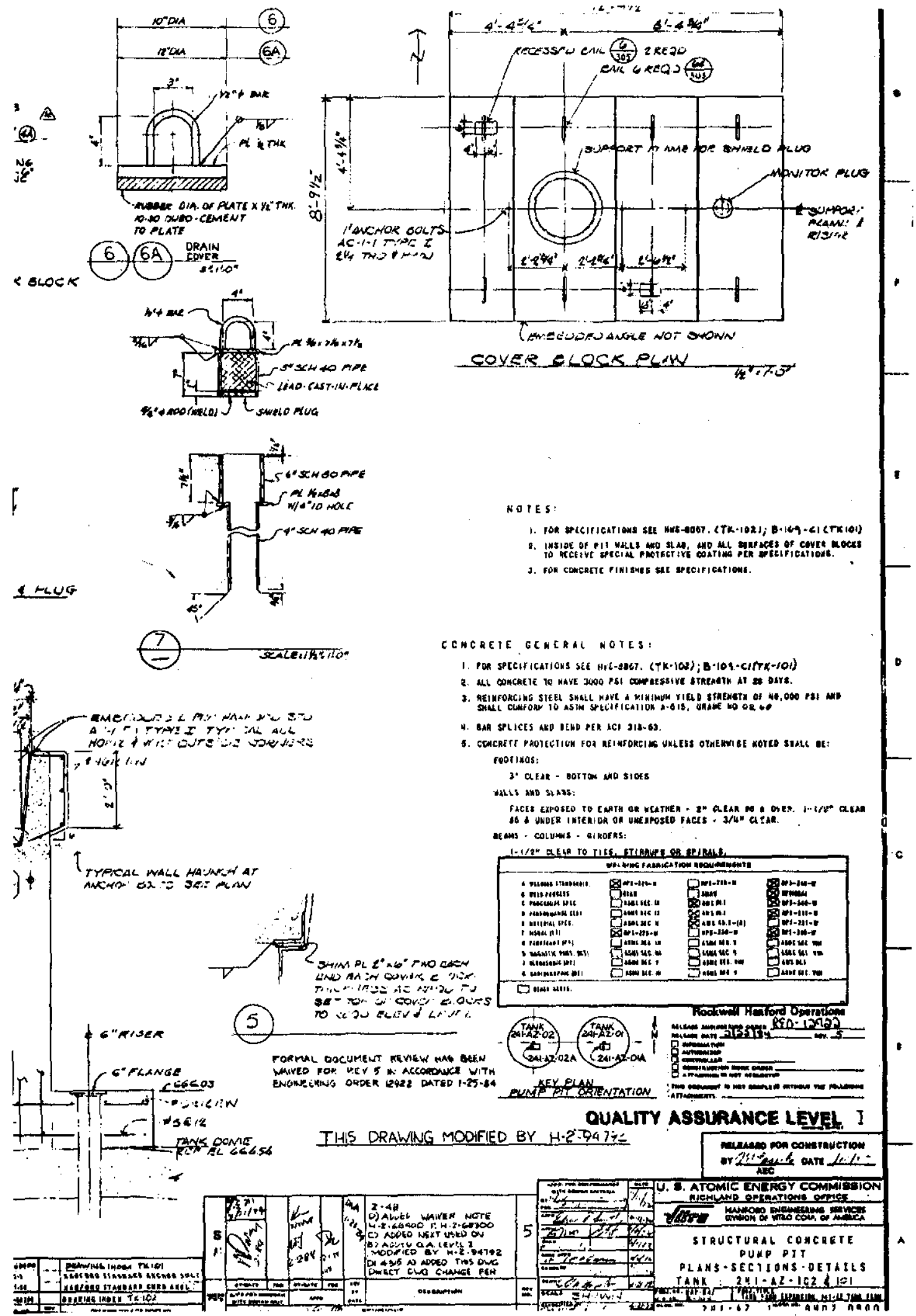


Figure A-6. Air Lift Circulator and Riser Extension Details - Part 1 of 2.

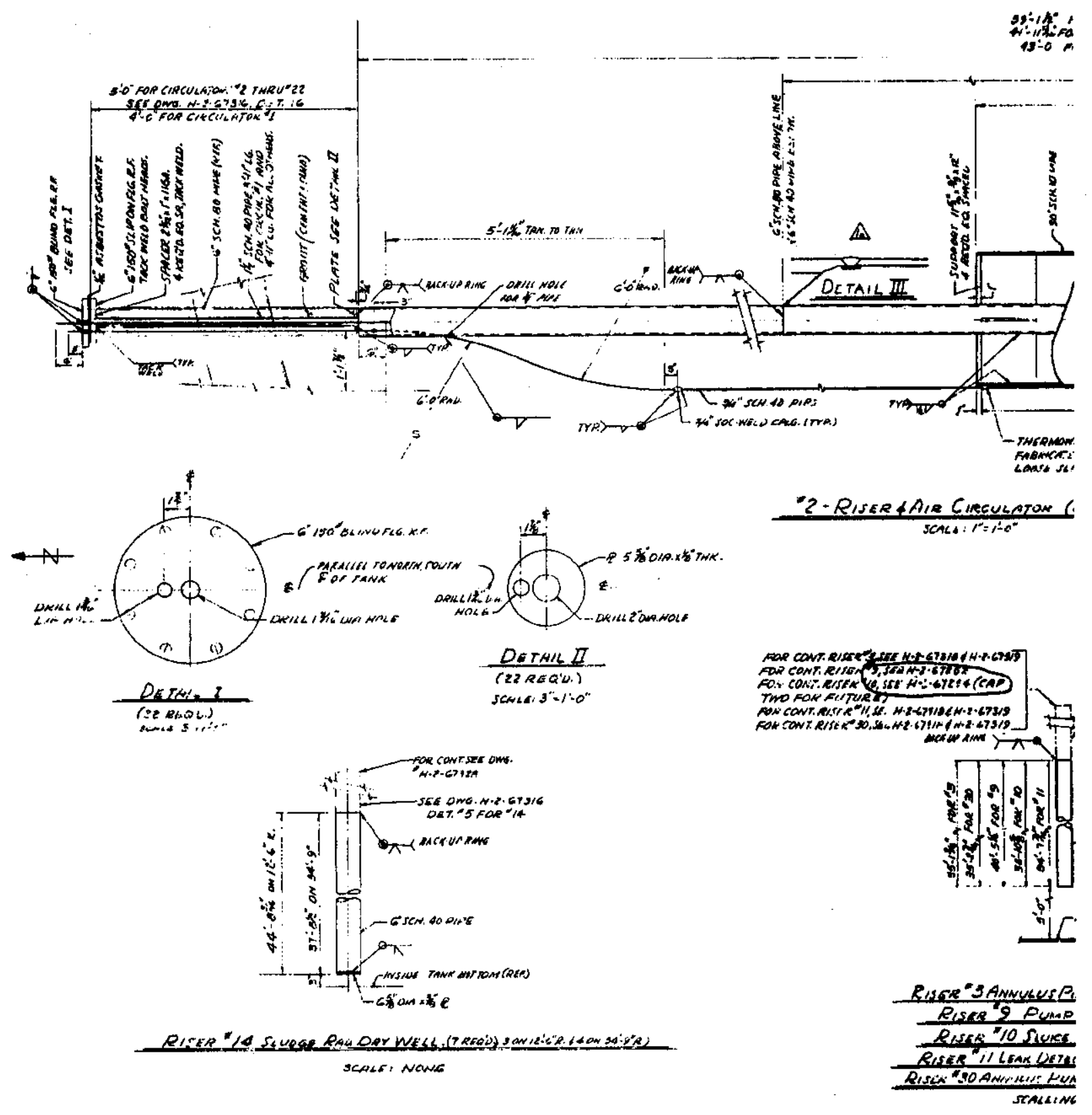




\section{RPP-6213 REV 0}

Figure A-7. Air Lift Circulator and Riser Extension Details - Part 2 of 2.
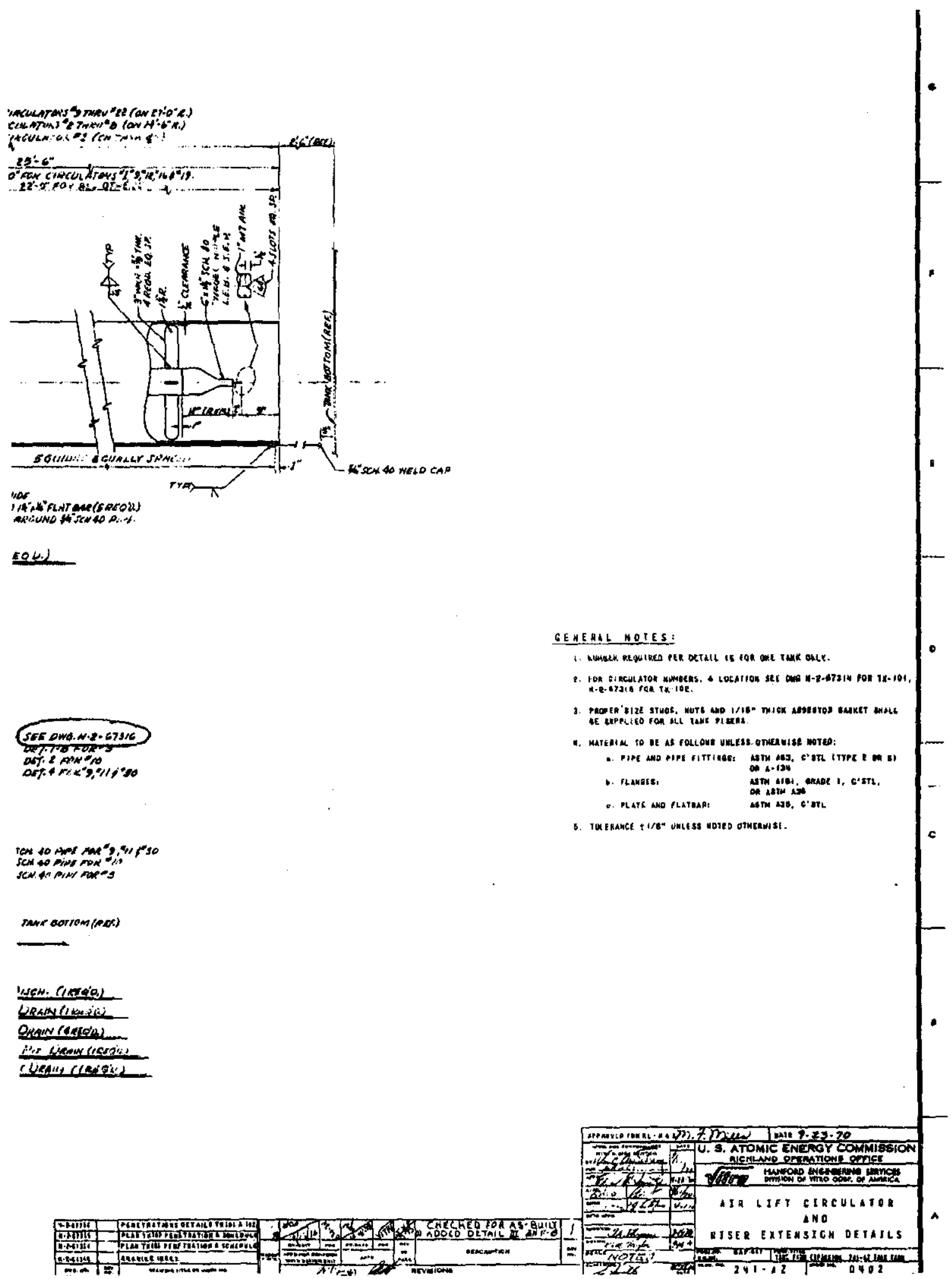

FAI/00-14, Rev. 0 
RPP-6213 REV 0

\section{A.3 TRANSMITTED DATABASE SPREADSHEET}




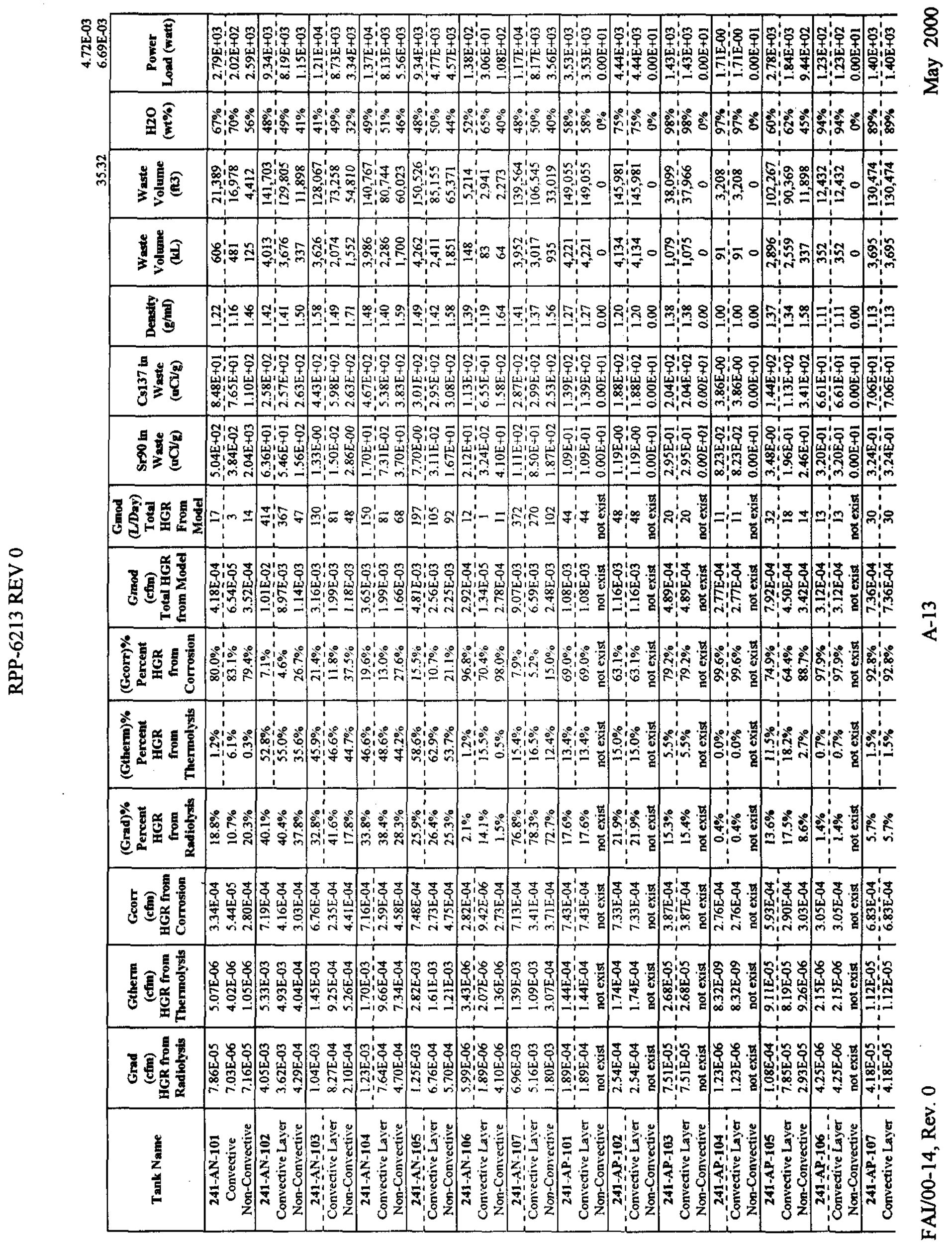




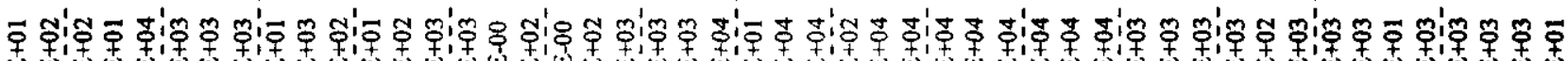

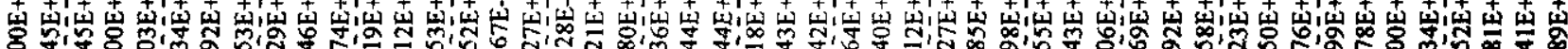
ơं

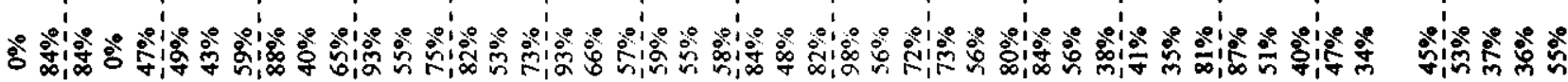

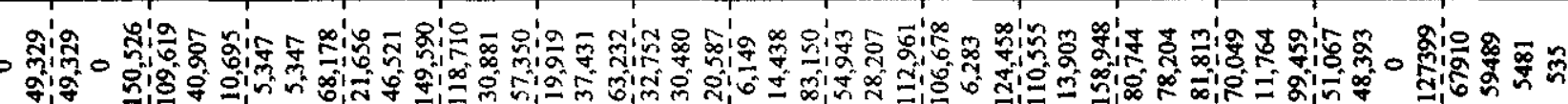
- 1 1

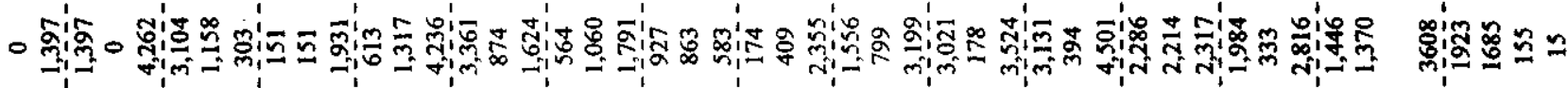

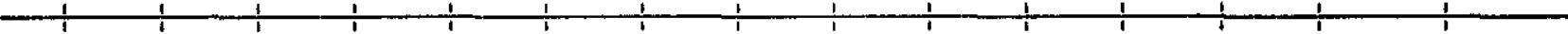

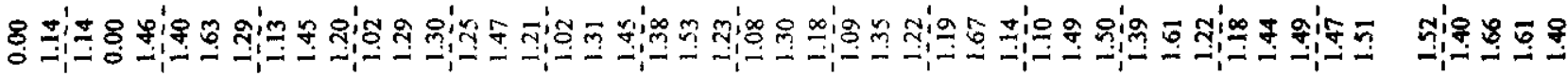

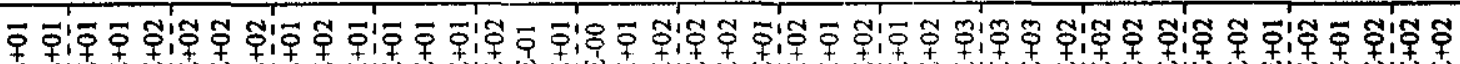

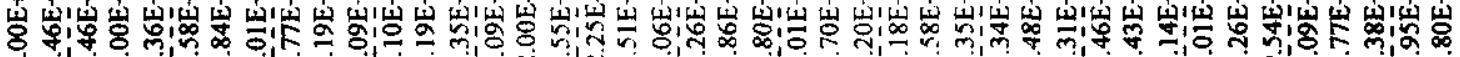

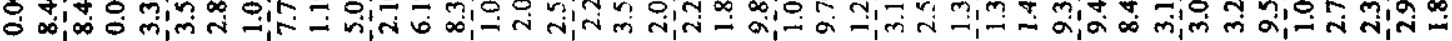

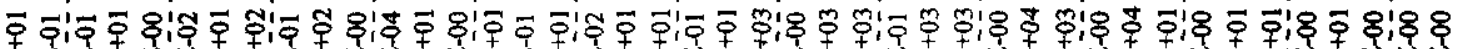

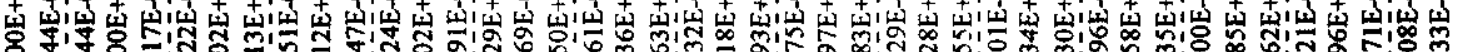
员 $\infty, \infty$ o

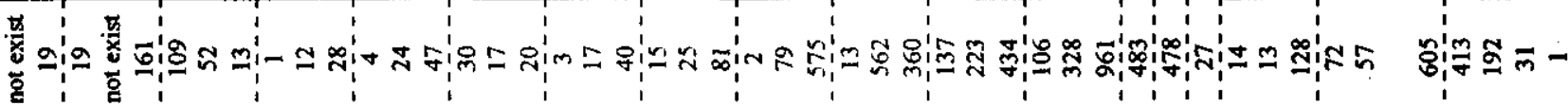

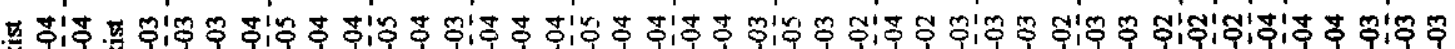

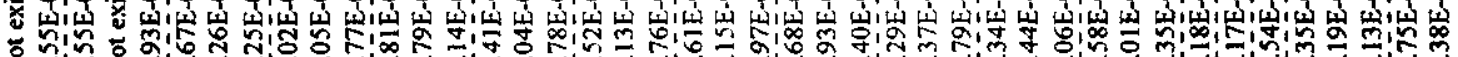

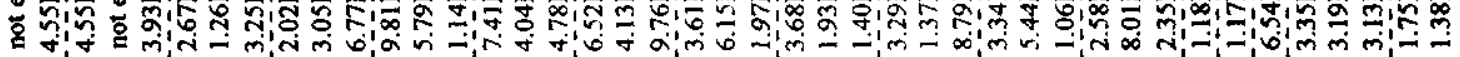

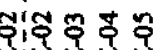

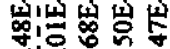

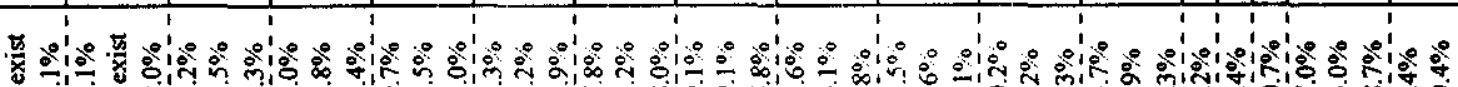

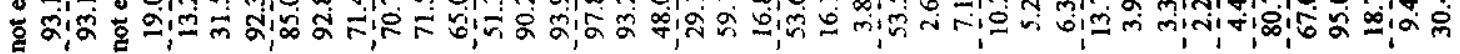

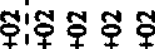
至衰点出

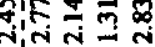

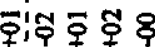

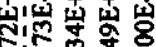

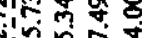
-

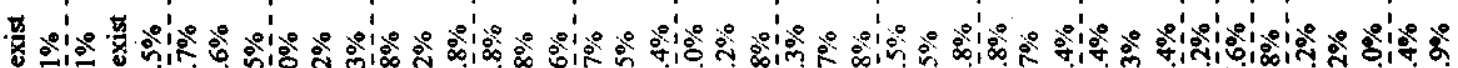

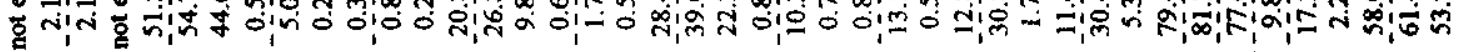

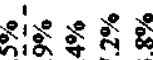
जia $\infty$ N

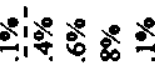

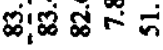

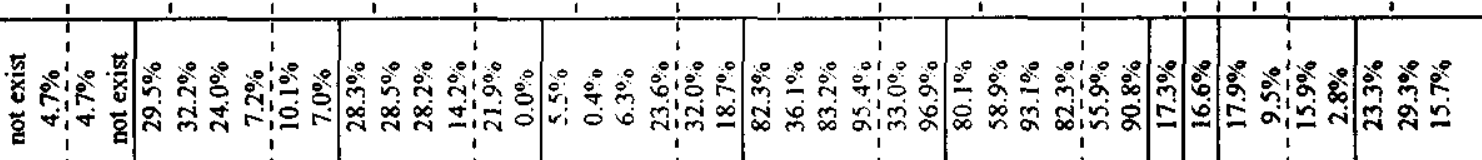

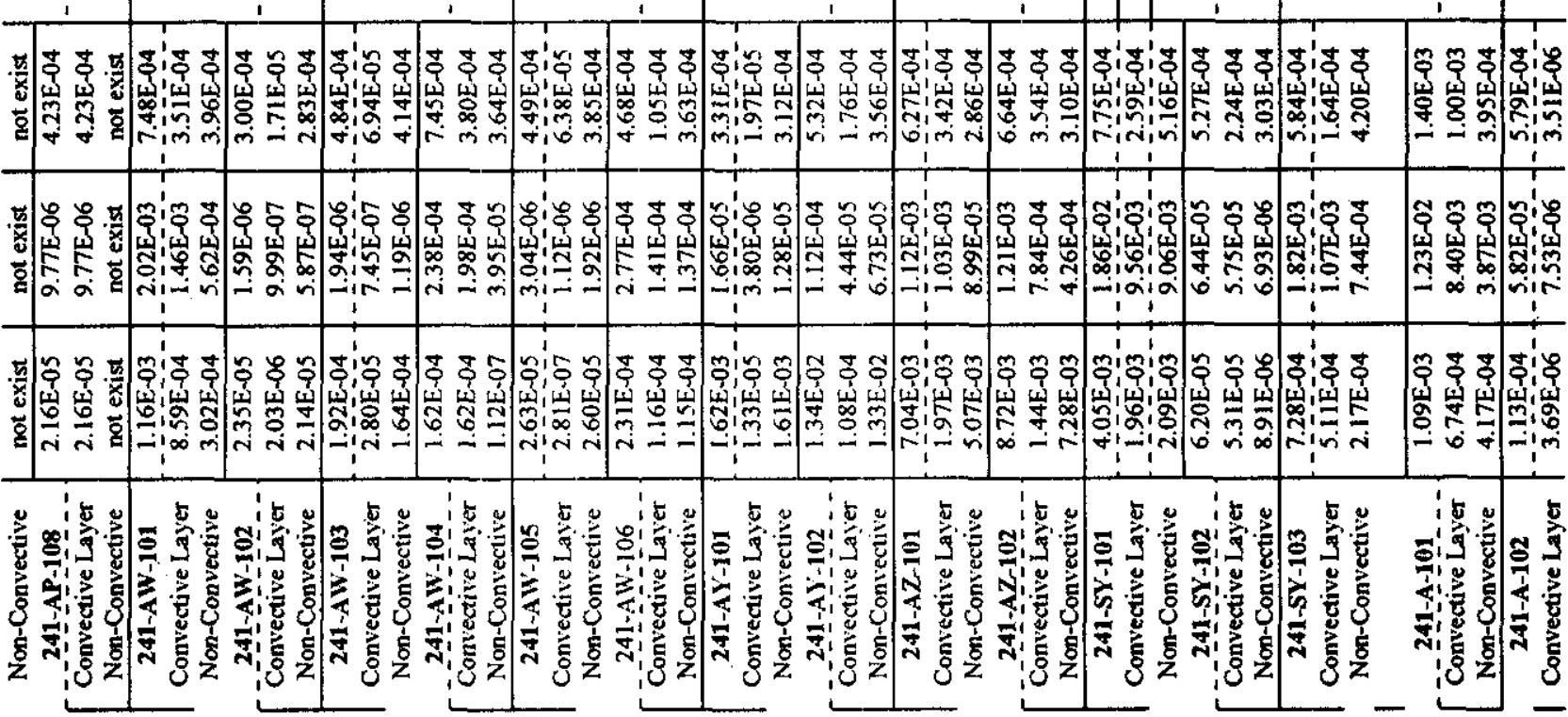

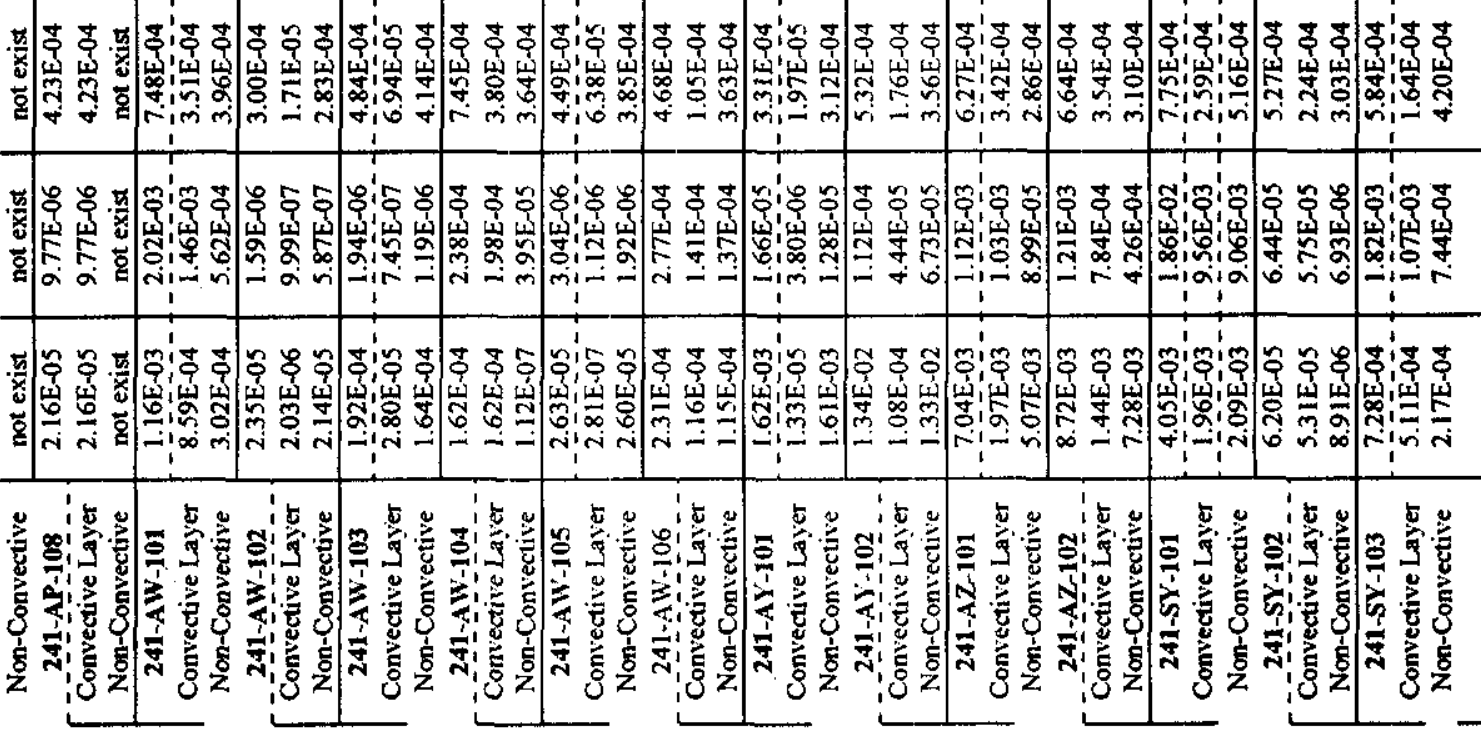




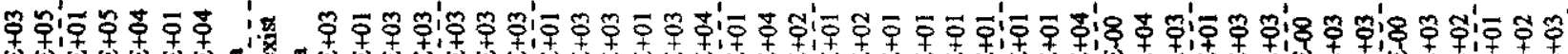

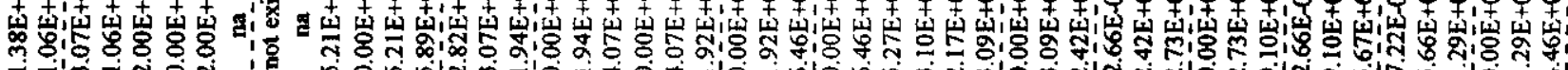
-

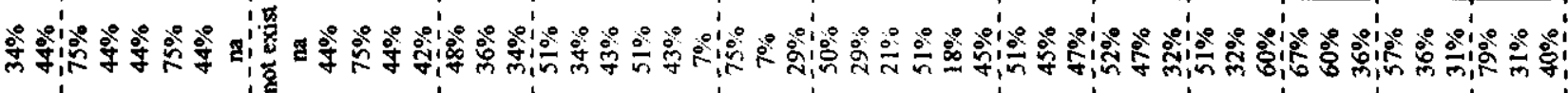

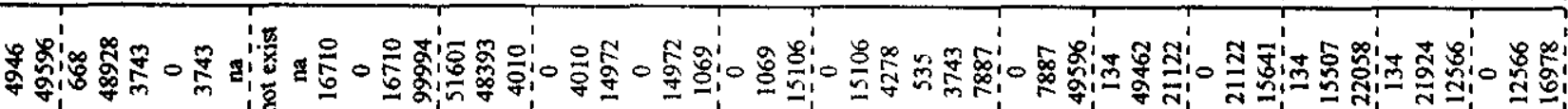

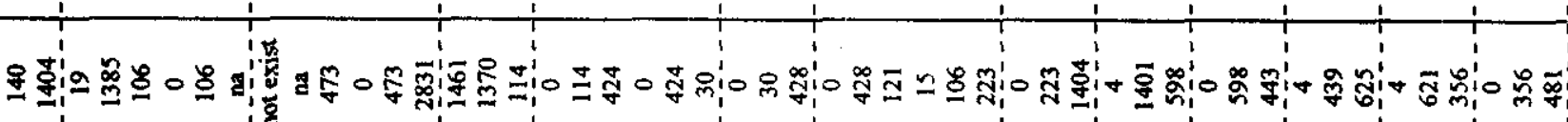

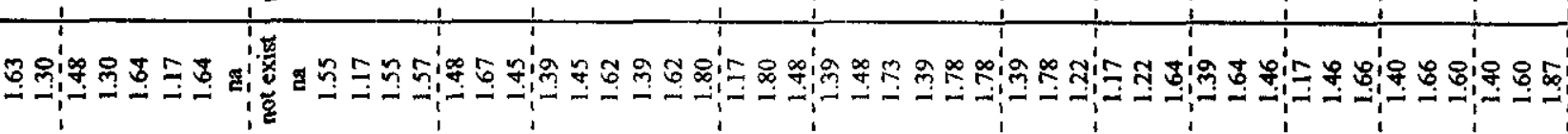

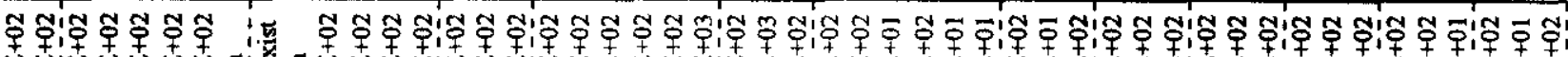

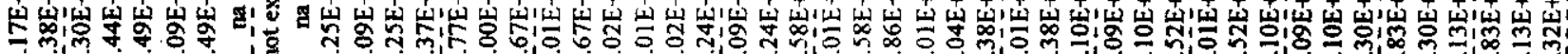
$\exists$ oin

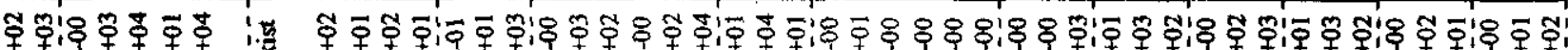

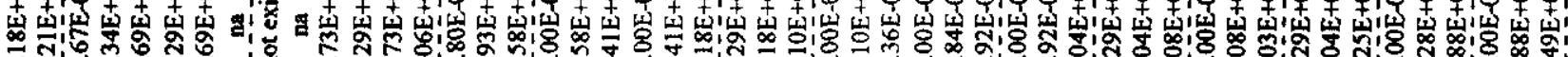
o

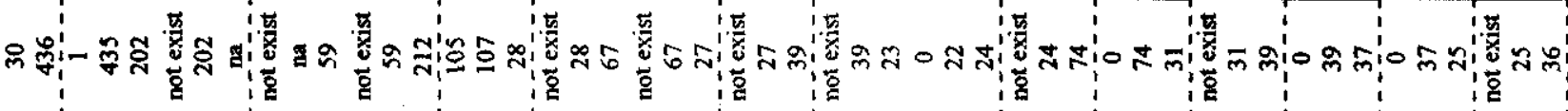

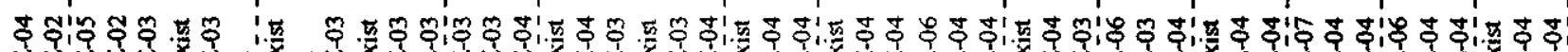

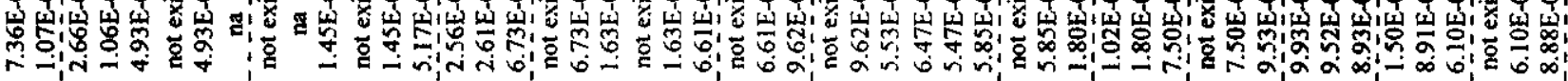

南

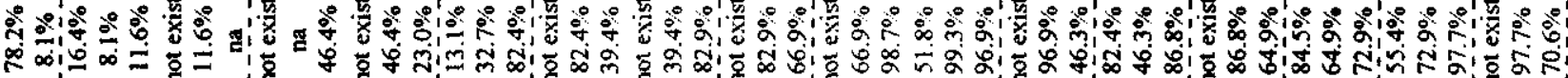

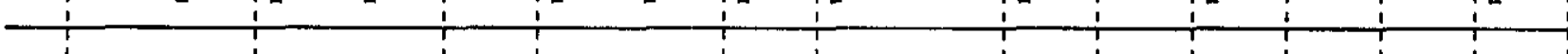
ร้ํำ 人,

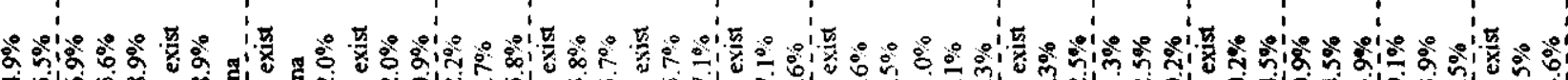

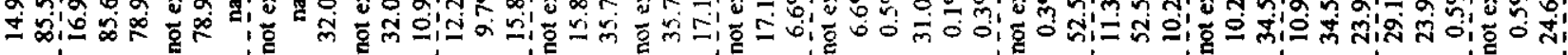

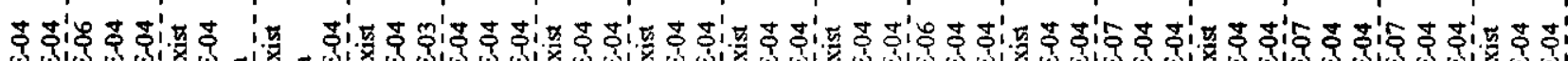

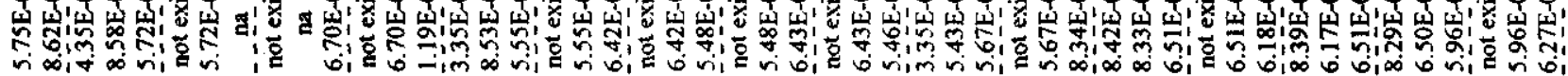

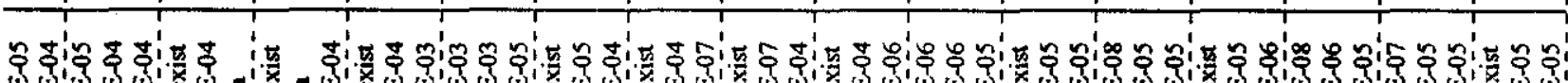

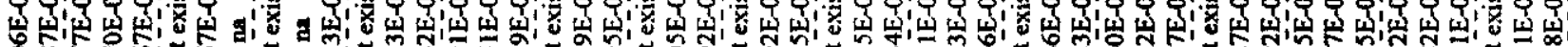

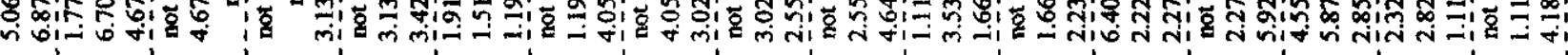

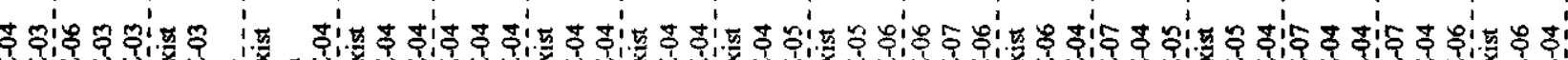

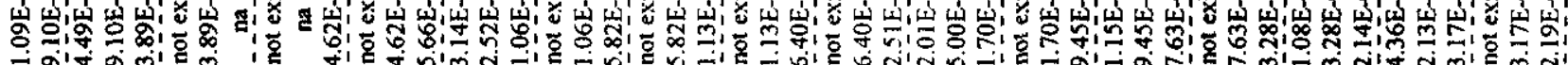
-

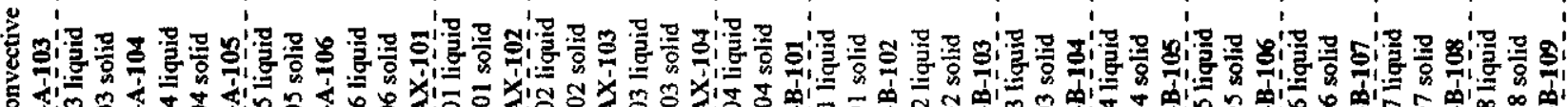

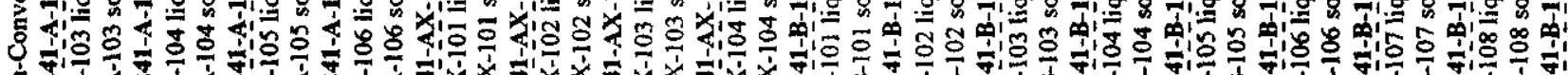

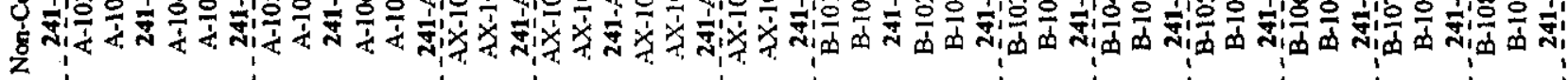




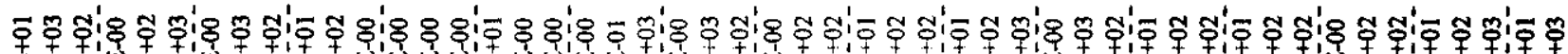

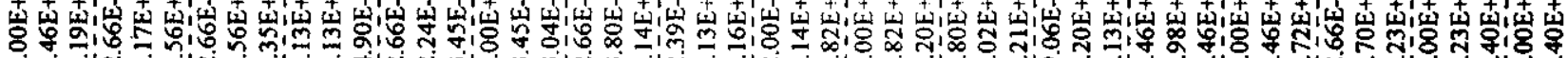

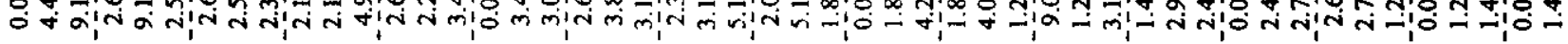

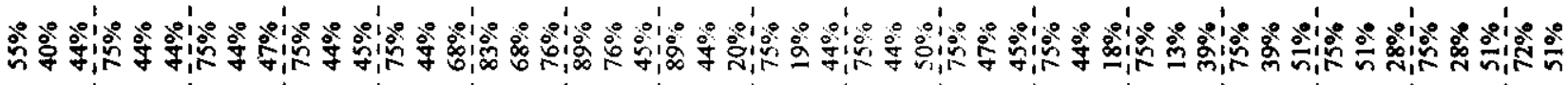

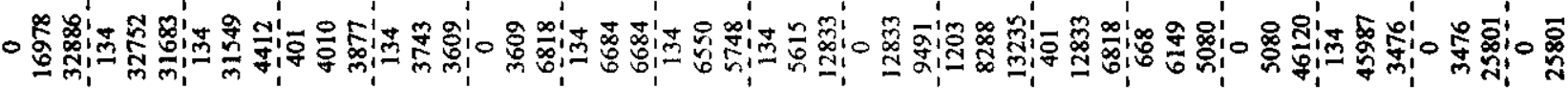

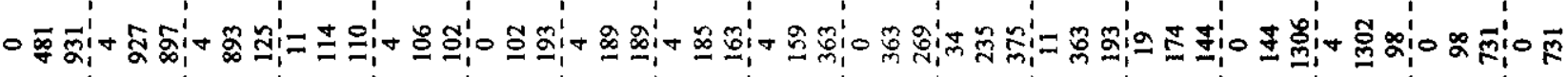

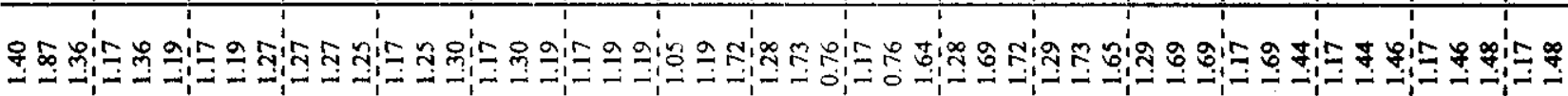

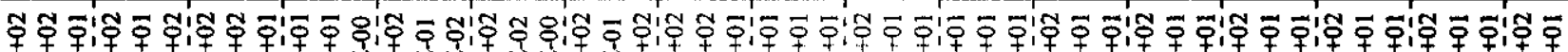

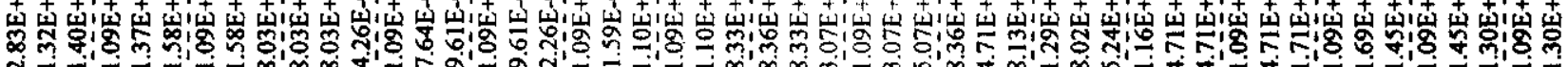

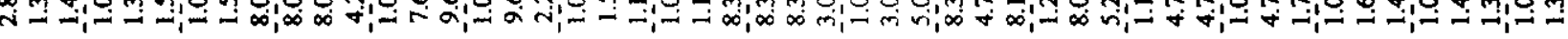

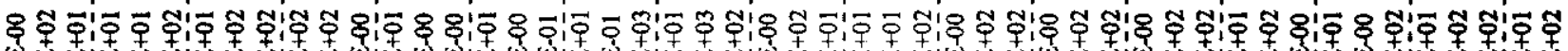

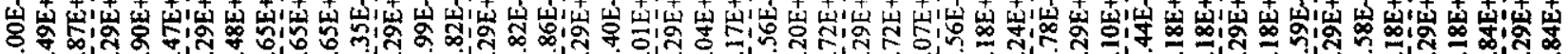
চ

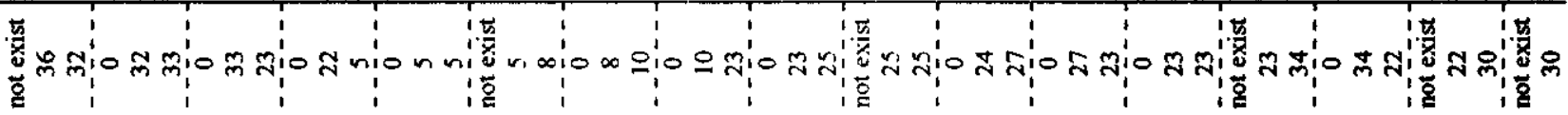

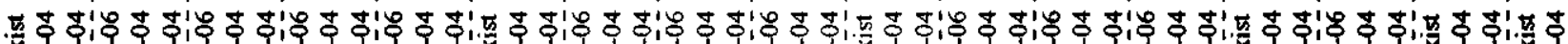

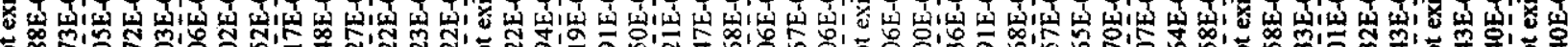
ơ

菨 ठำ

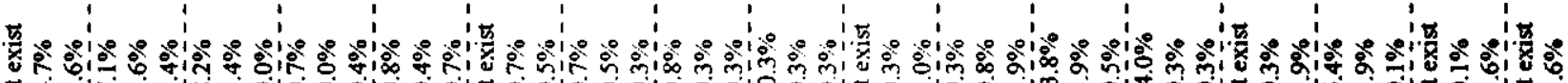
远 -

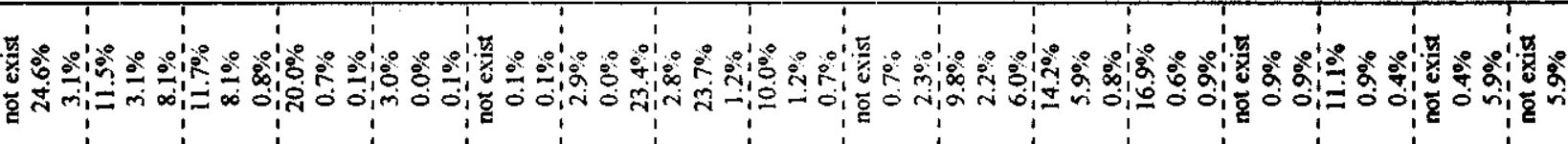

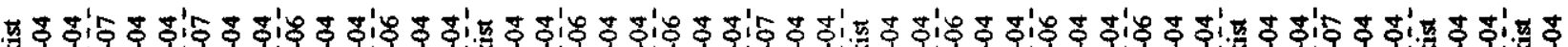

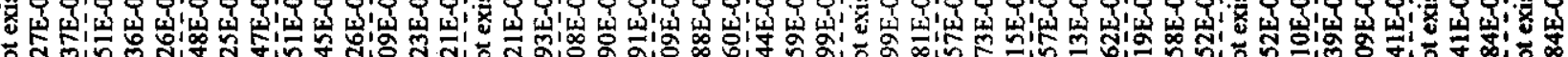

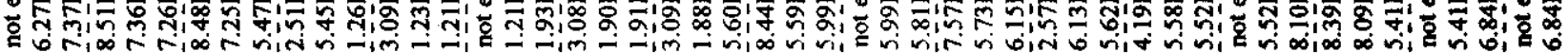

"

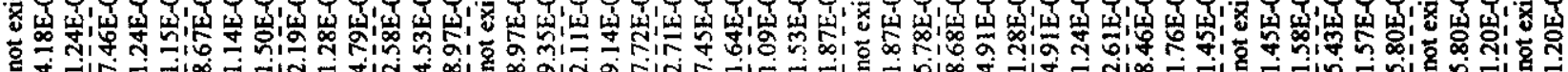

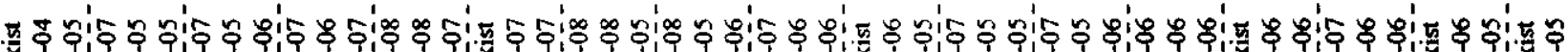

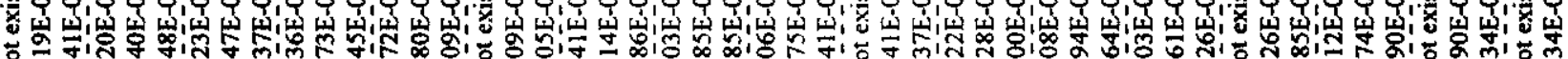

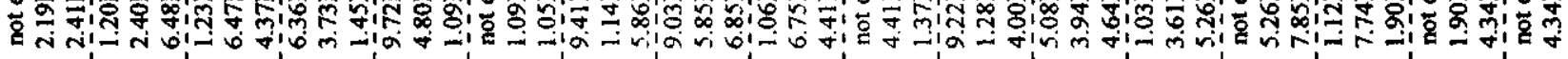

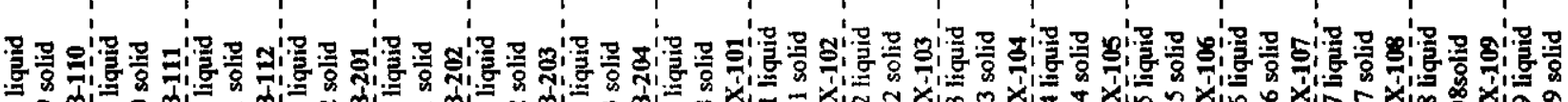

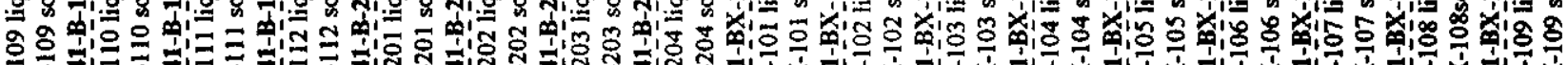

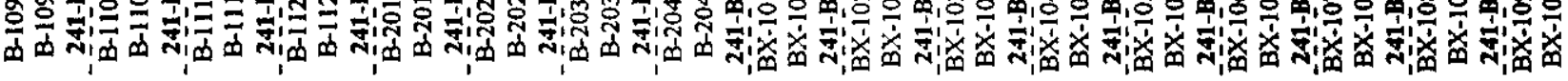




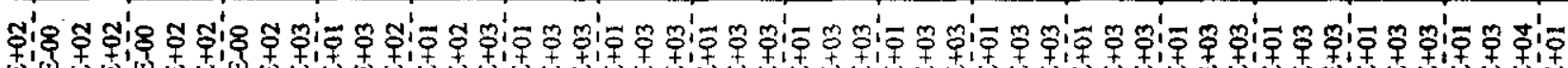

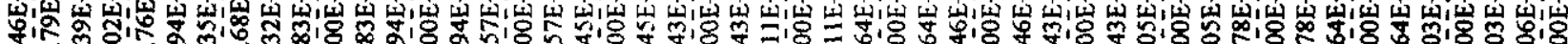
नi, N

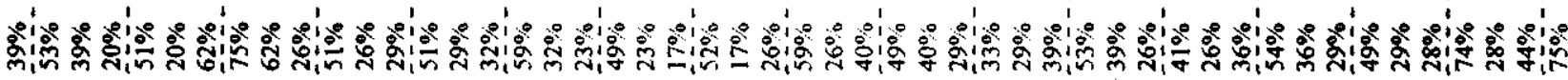

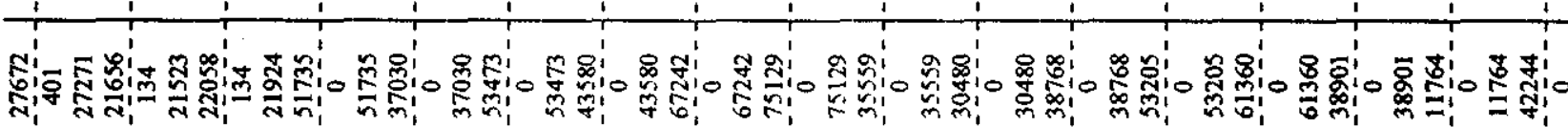

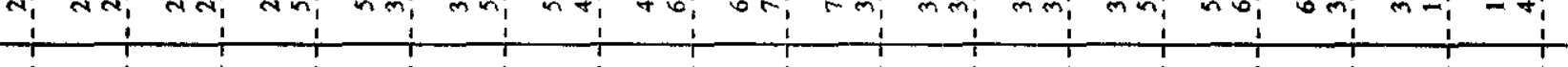

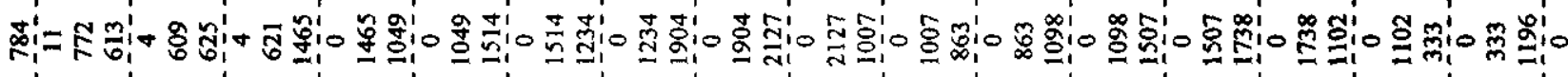

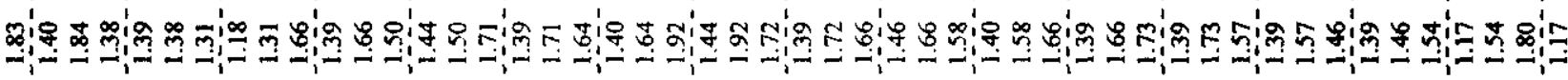

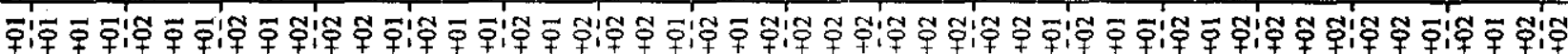

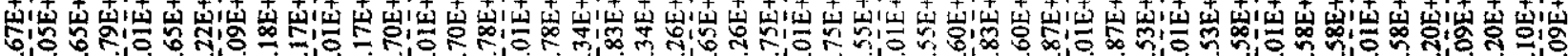

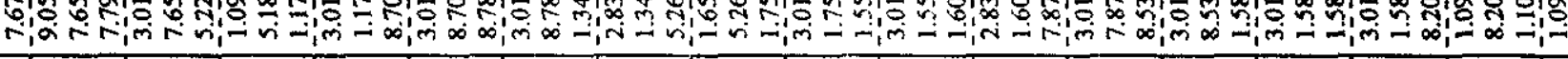

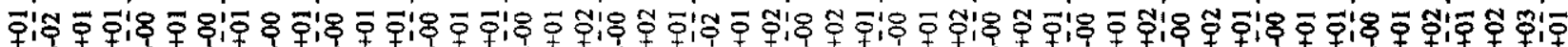

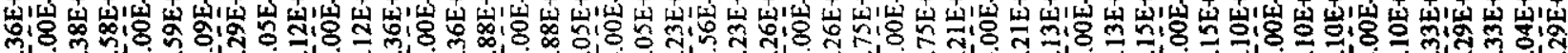

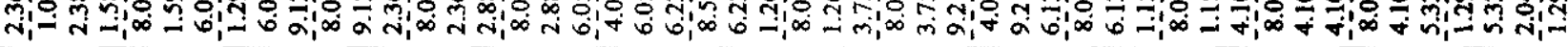

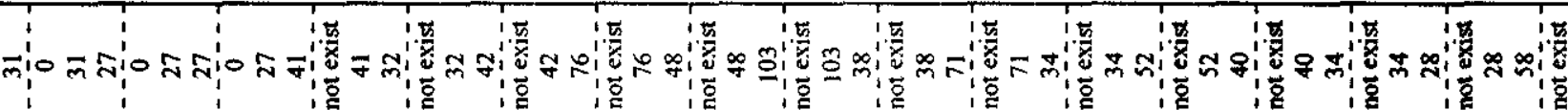

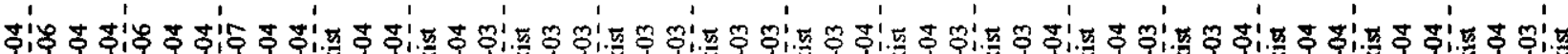

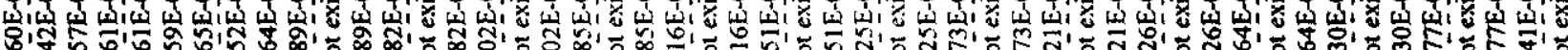

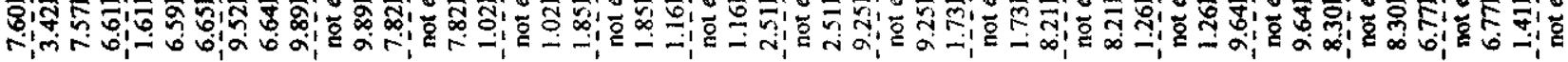

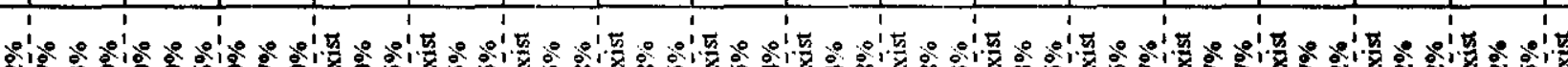
न! !

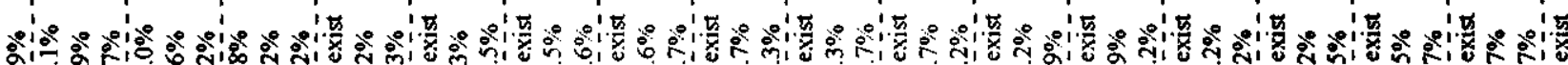

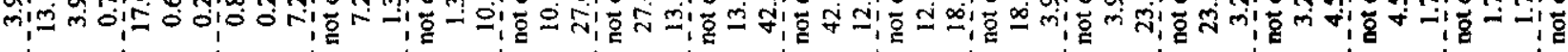

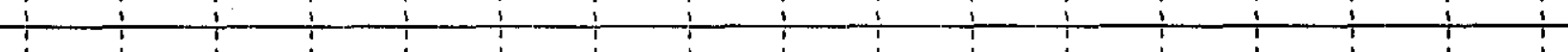

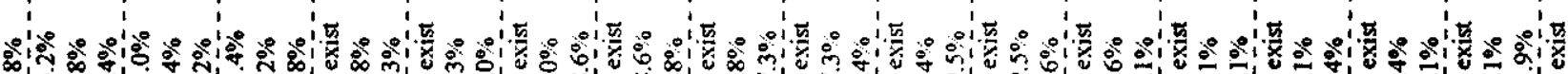

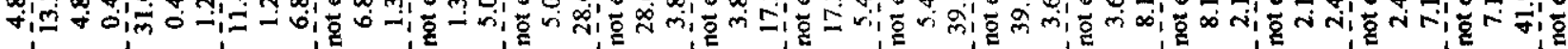

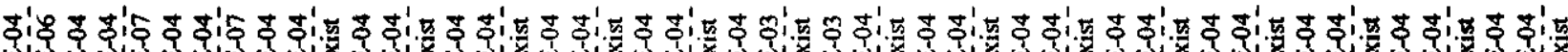

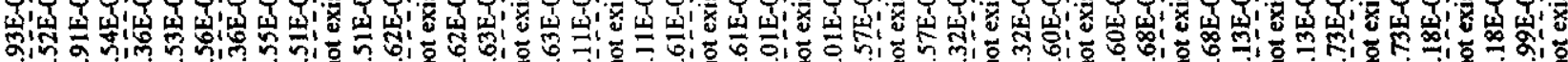
бin

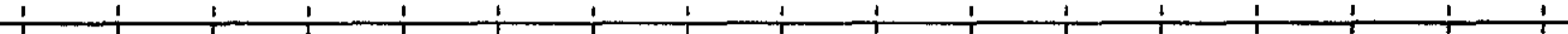

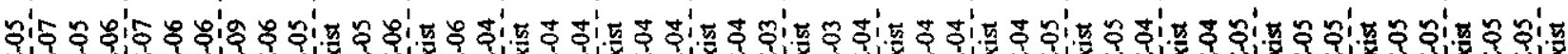

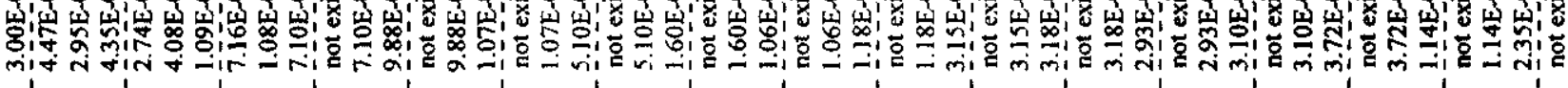

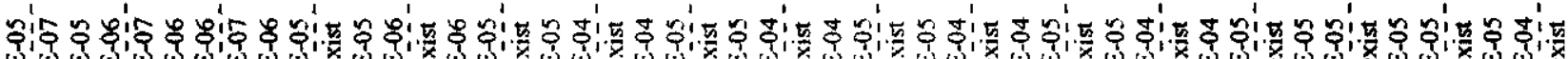

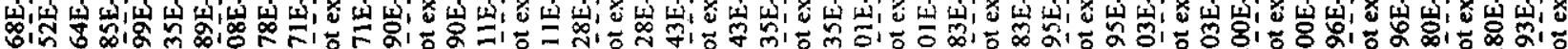

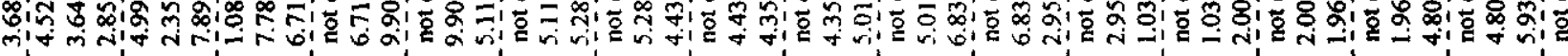

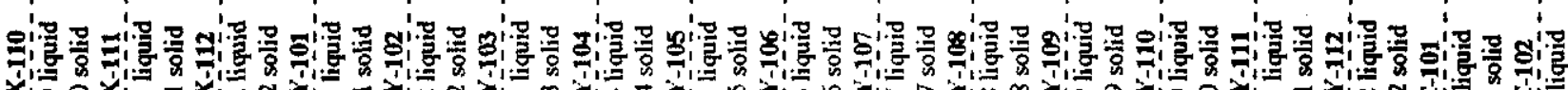

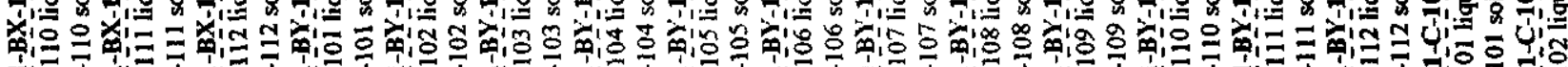

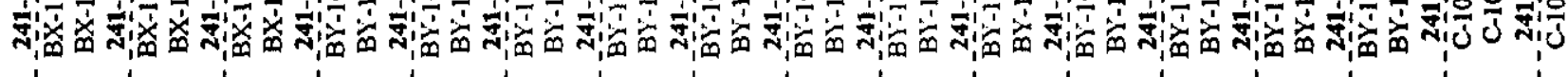




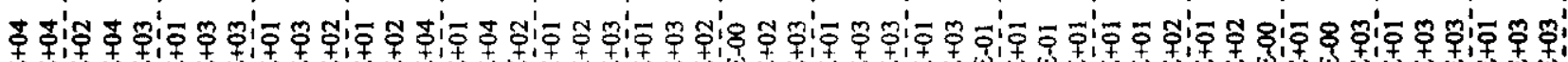

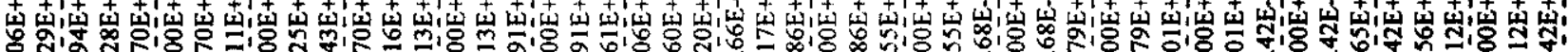

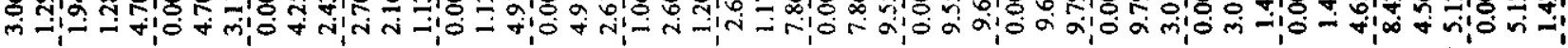

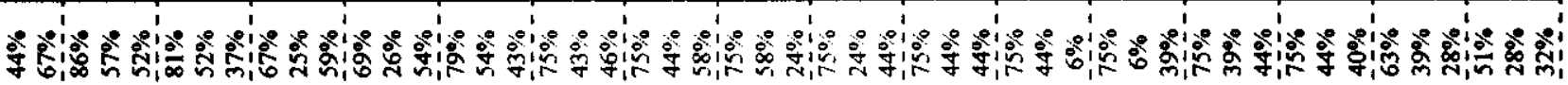

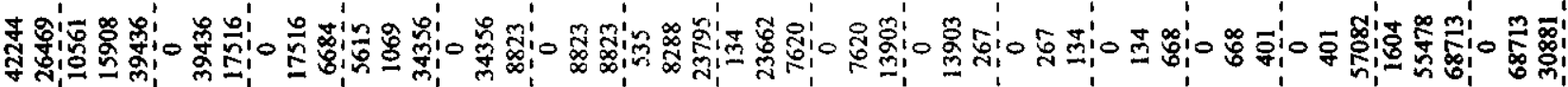

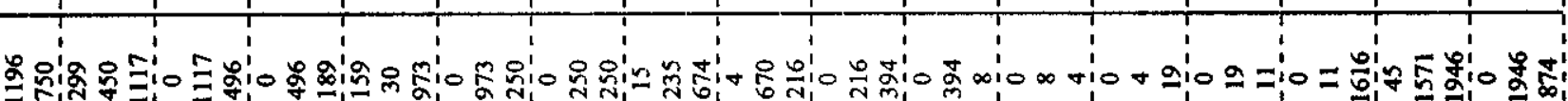

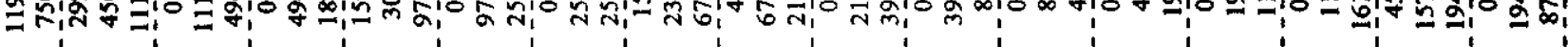

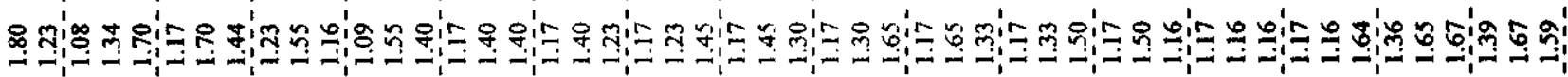

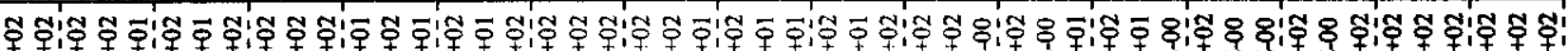

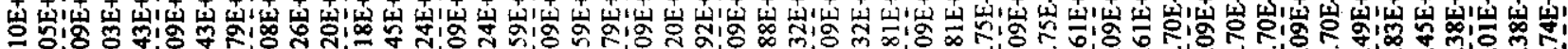

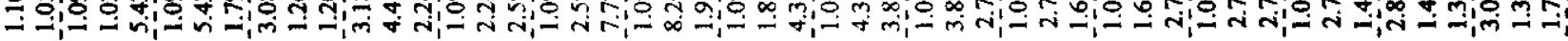

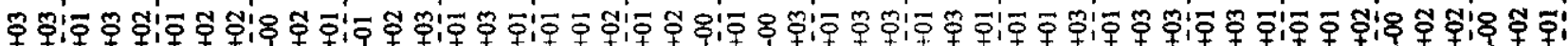

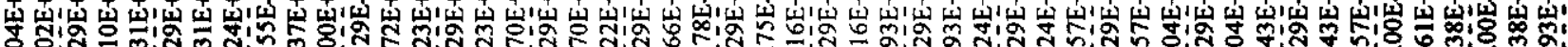

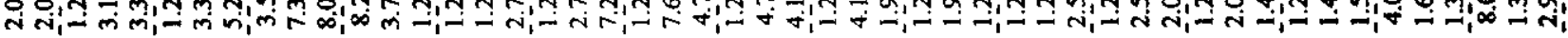

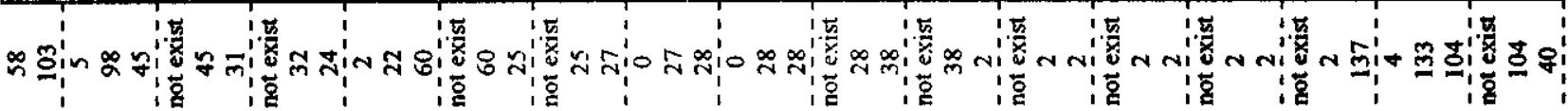

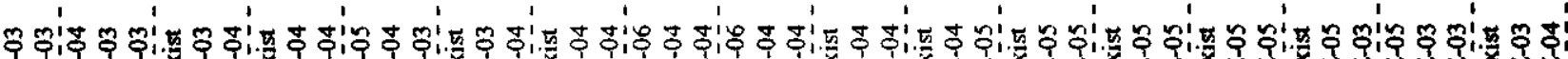

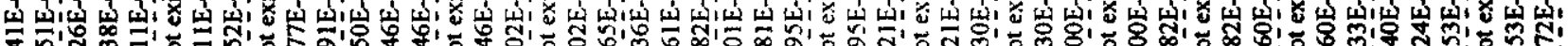

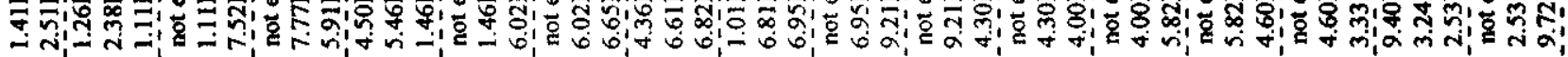

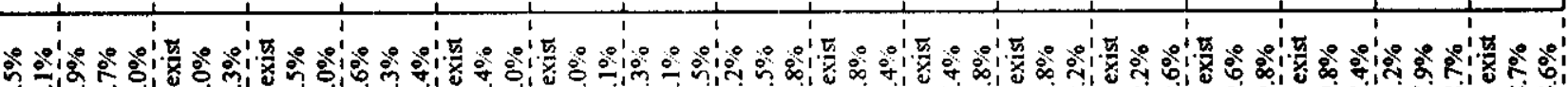

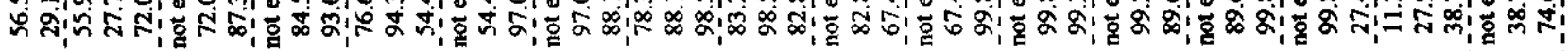

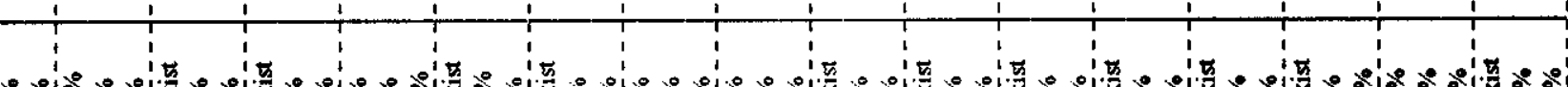

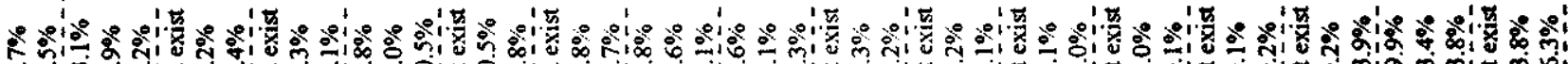
=

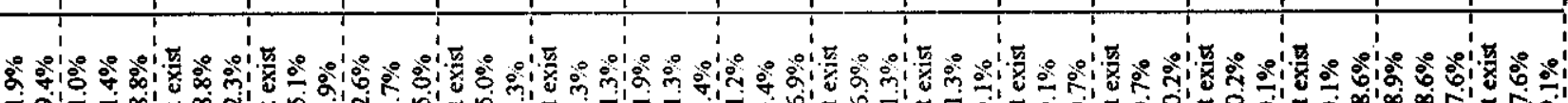

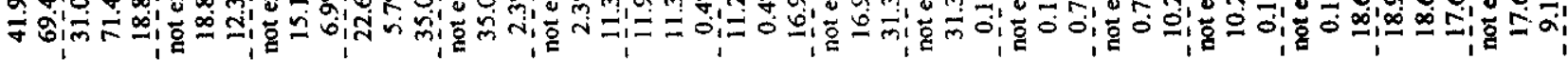

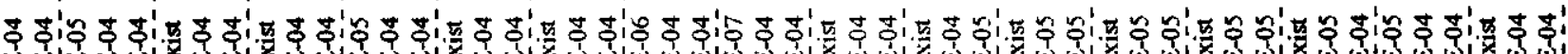

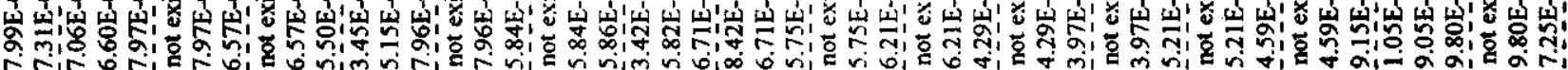

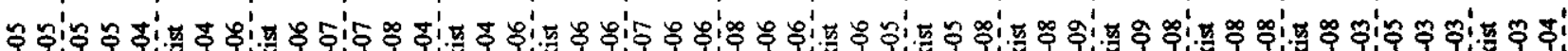

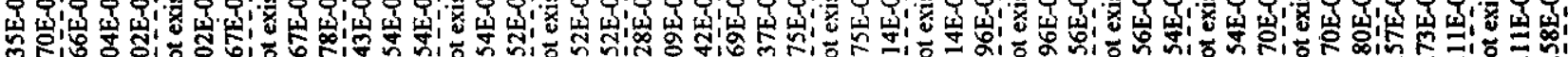

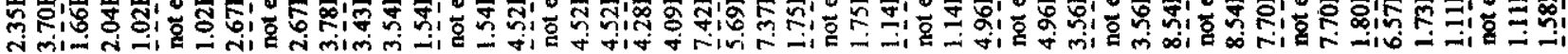

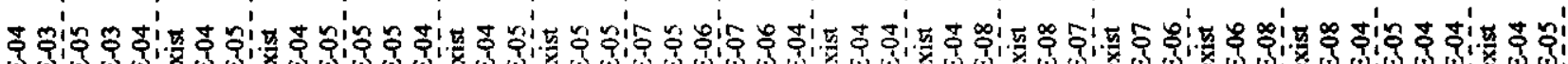

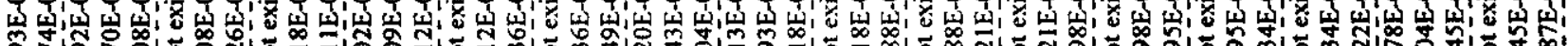

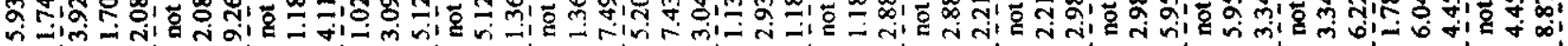

(10

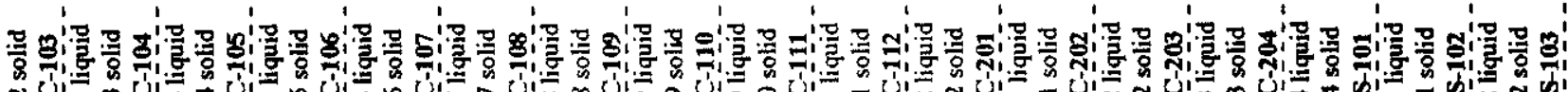

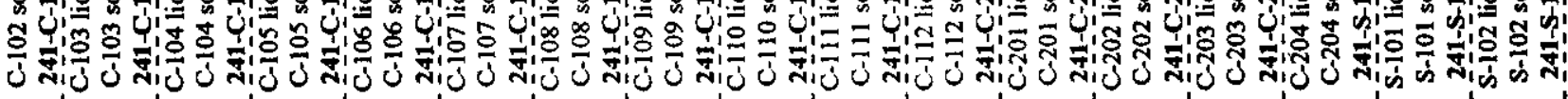




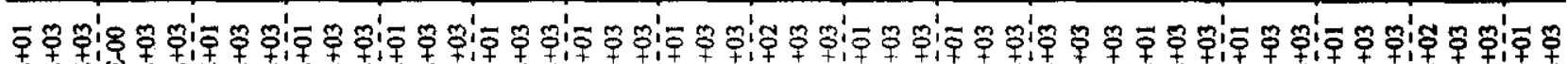

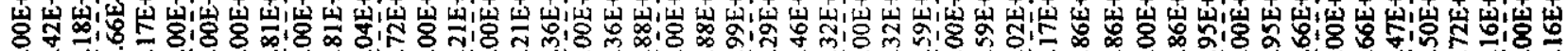
Ö -

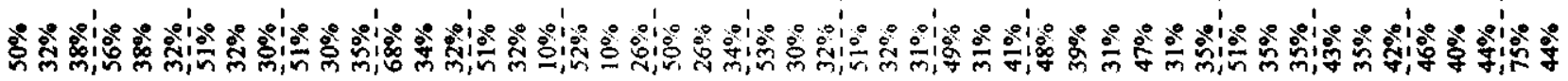

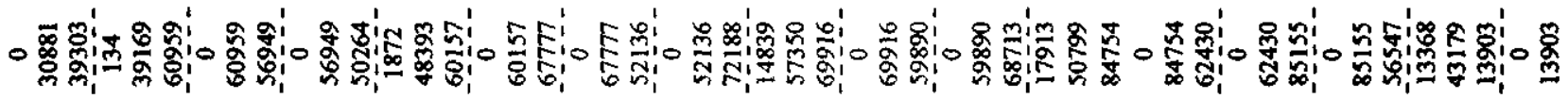

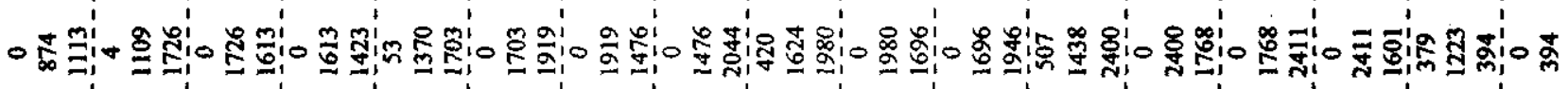

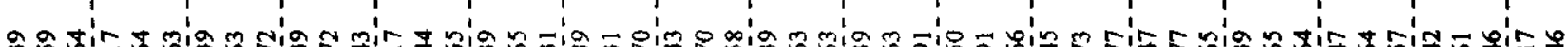

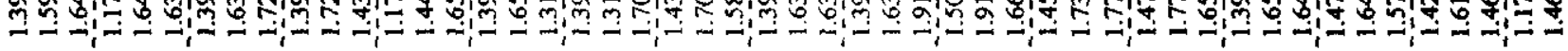

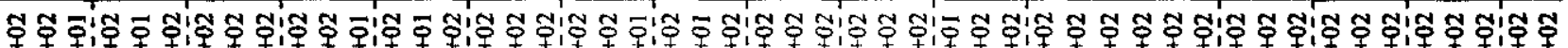

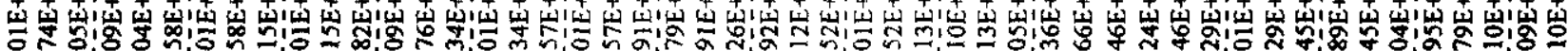

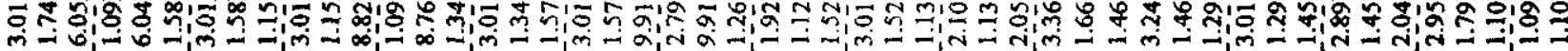

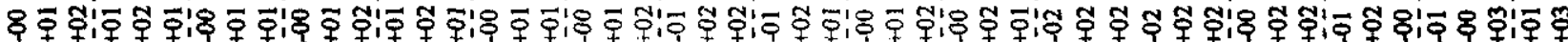

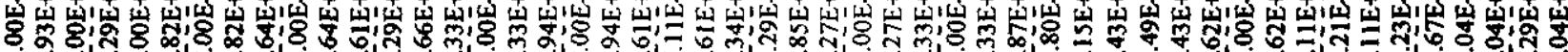

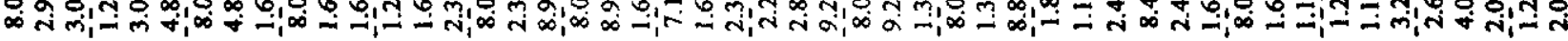

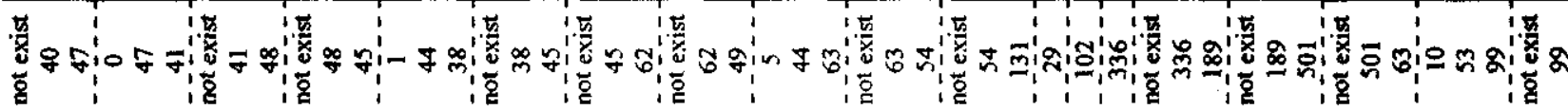

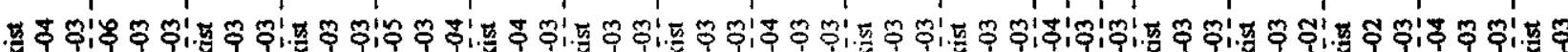

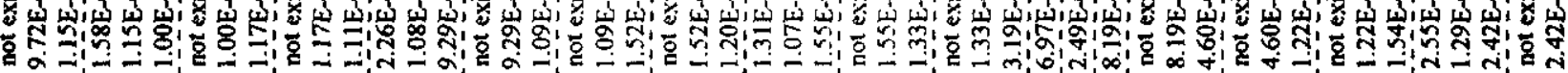

le

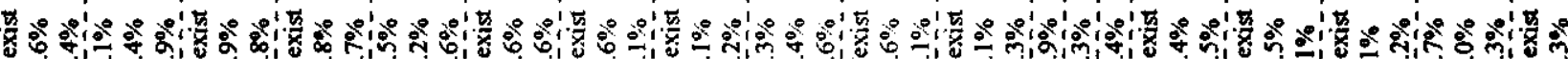

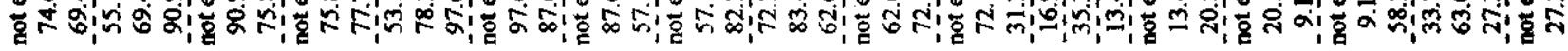

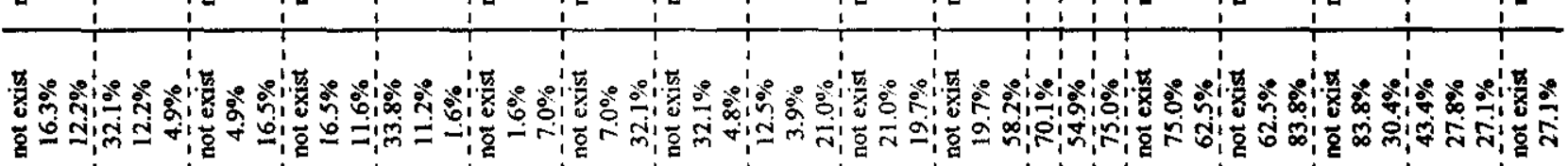

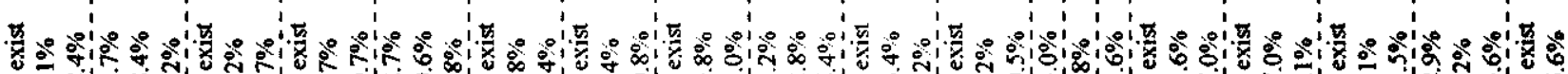

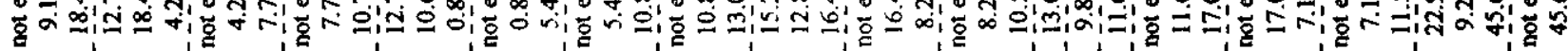

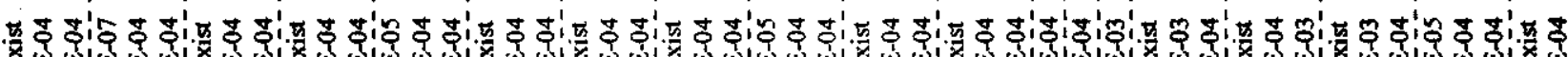

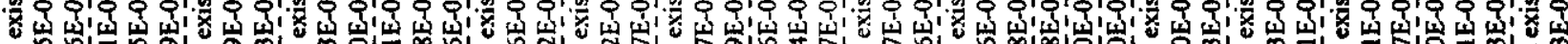

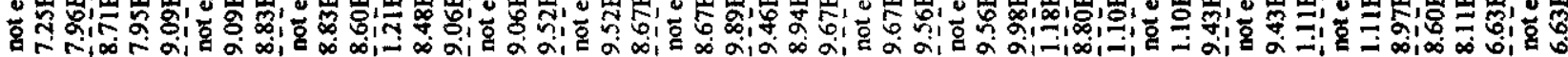

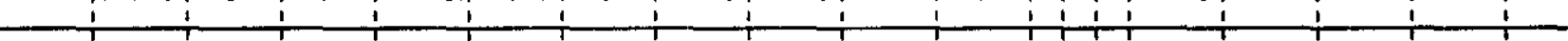

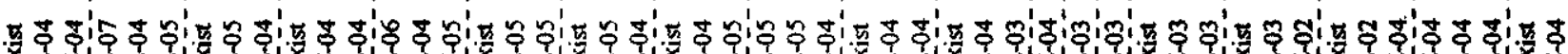

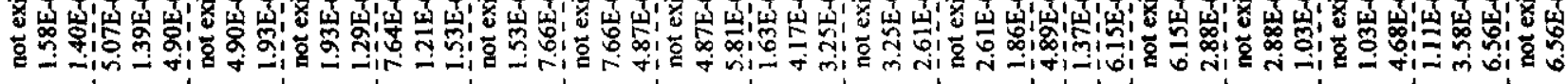

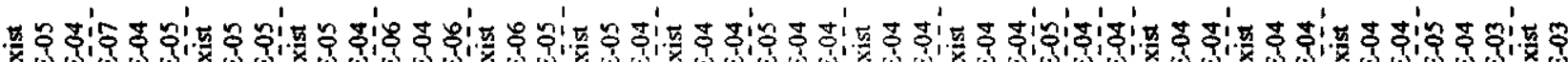

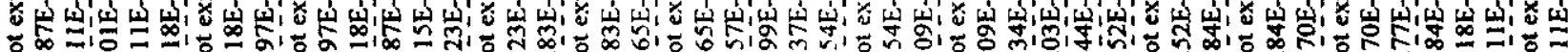
ơ

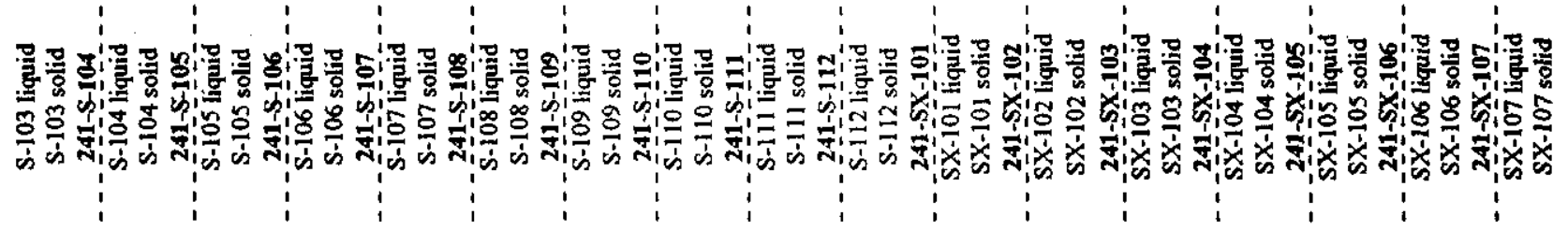


This page cannot be converted.

Please view the native document

for the original page. 


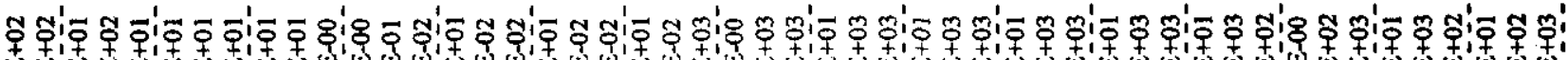

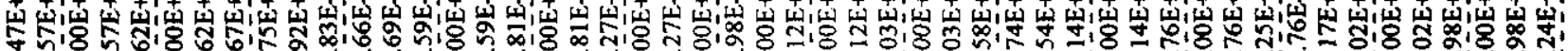
molo

خิ

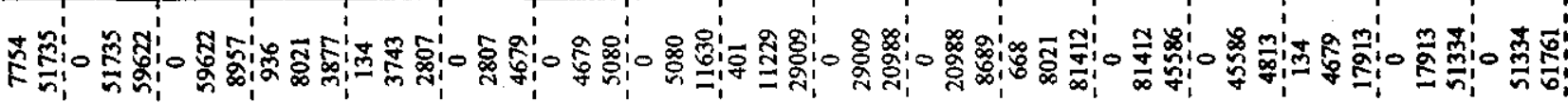

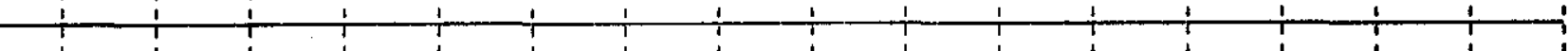

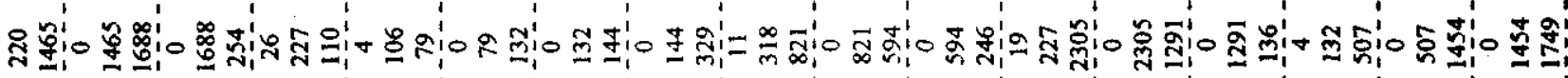

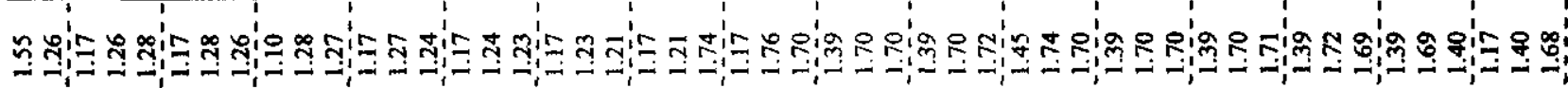

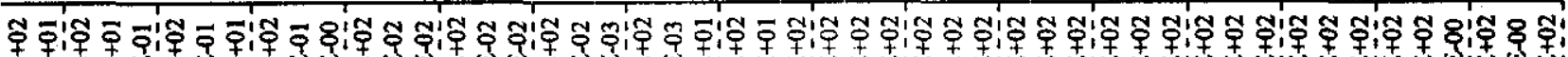

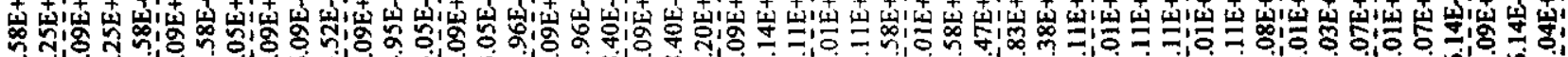

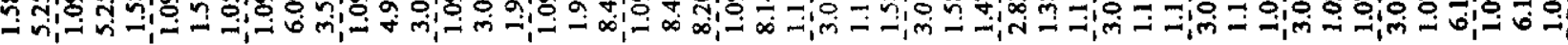

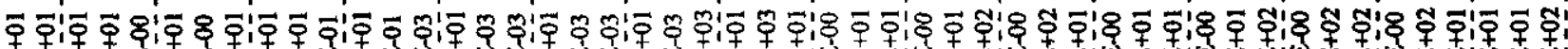

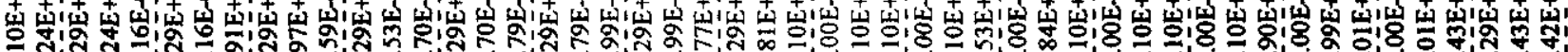

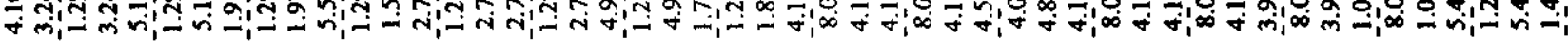

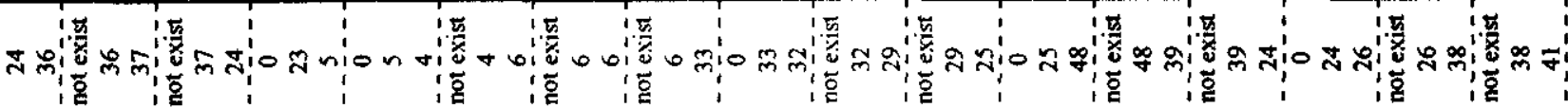

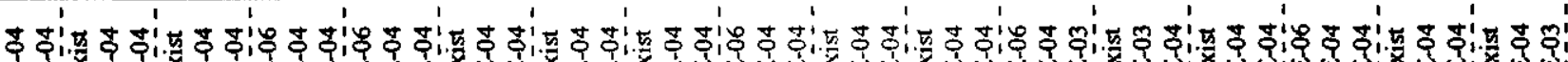

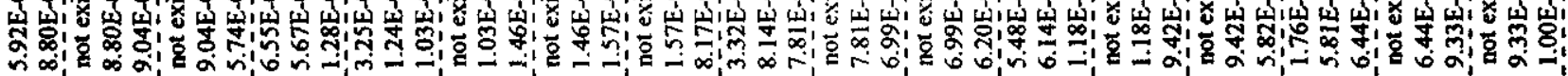

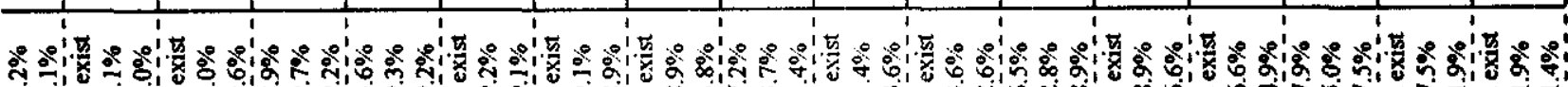

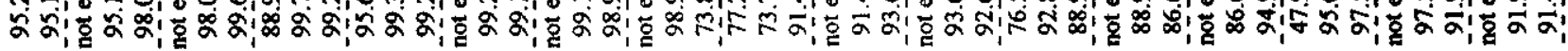

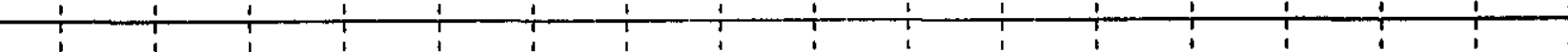

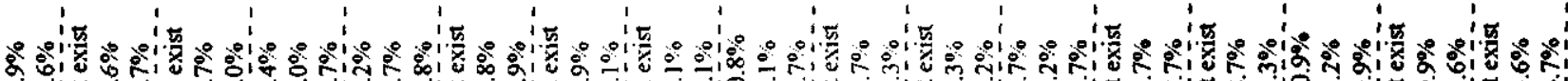

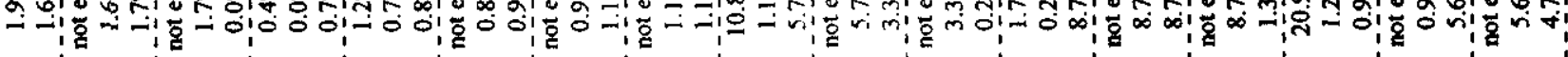

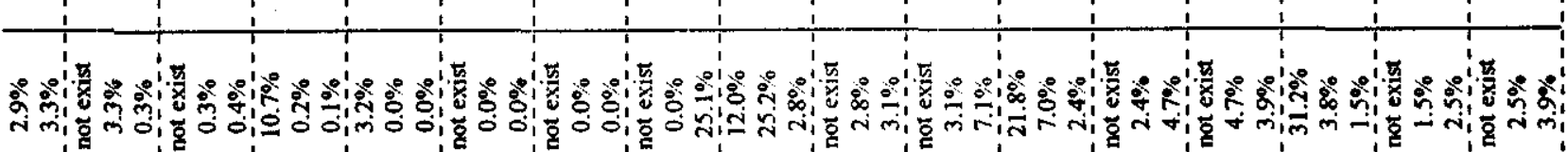

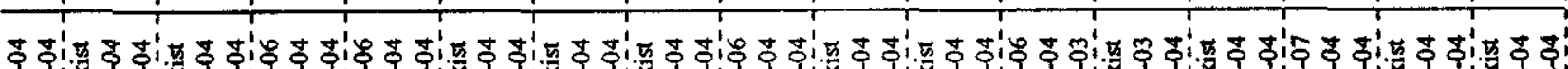

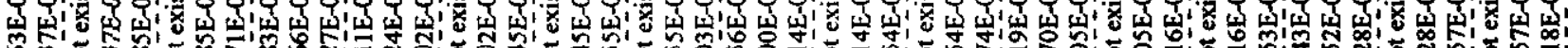

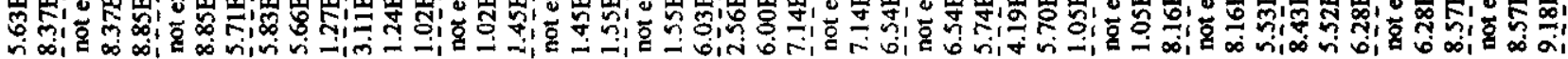

\begin{tabular}{l}
1 \\
\hline
\end{tabular}

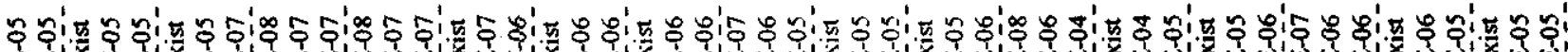

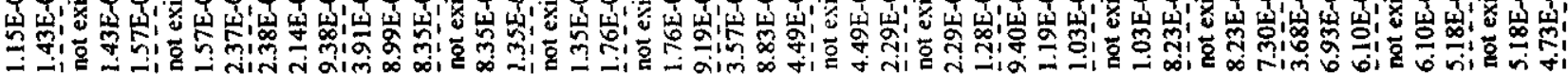

彳 ni.

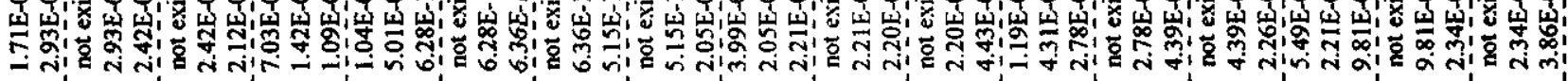

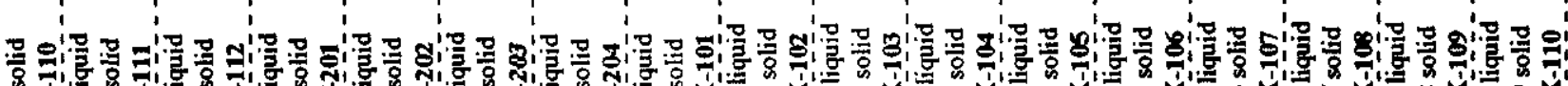

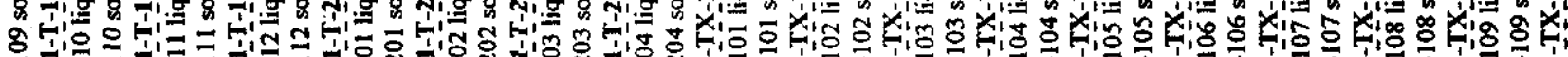

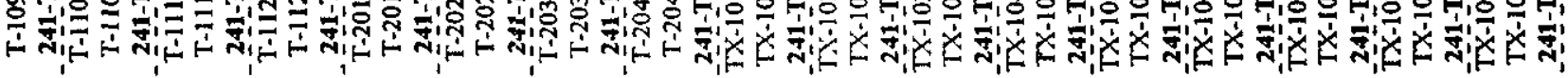




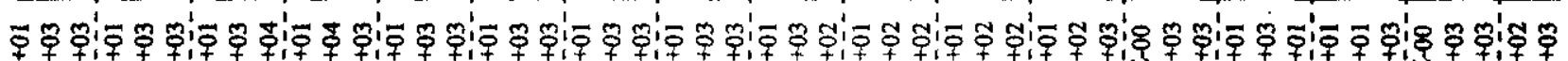

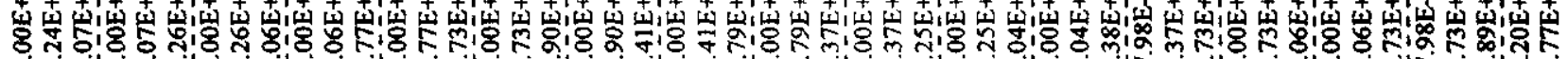

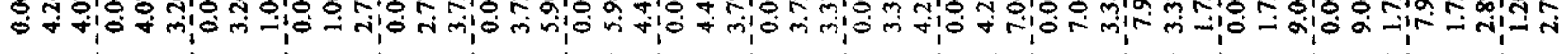

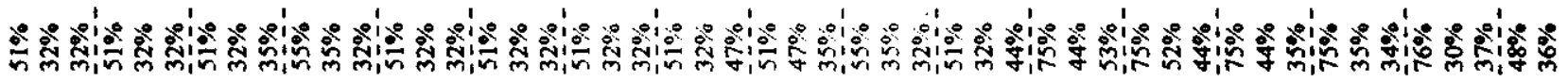

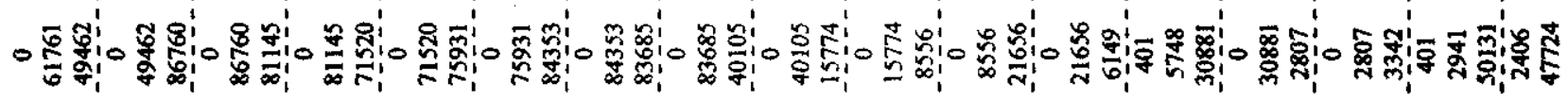
-

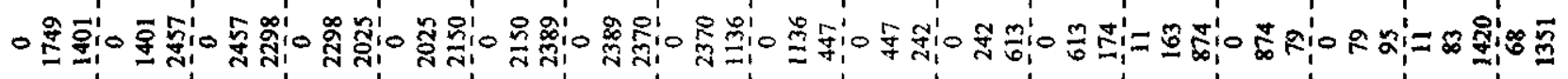
음

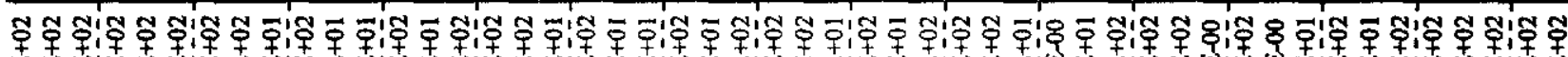

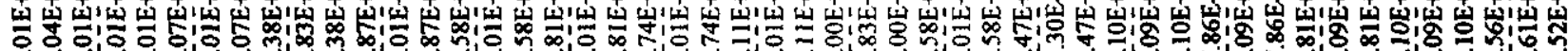

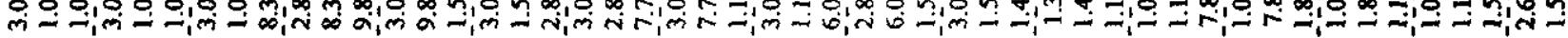

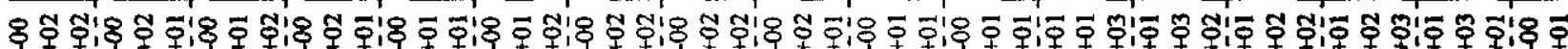

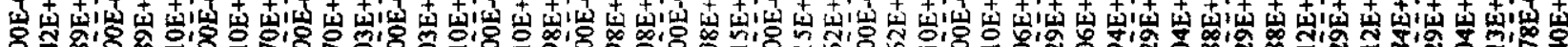

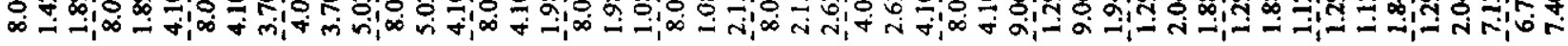

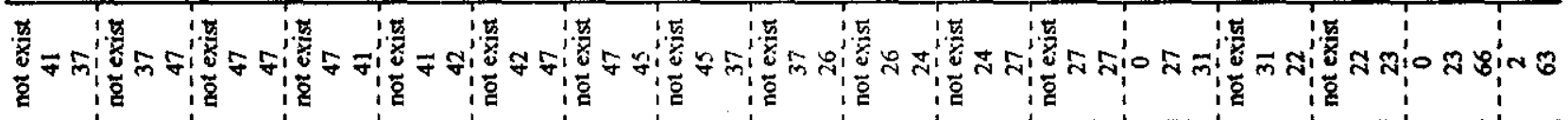

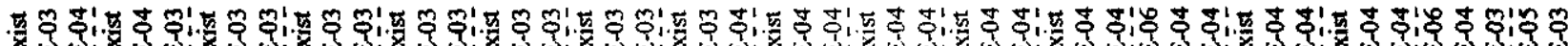
䔁

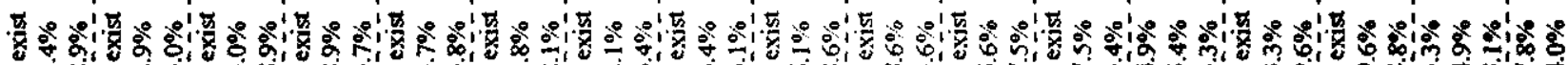

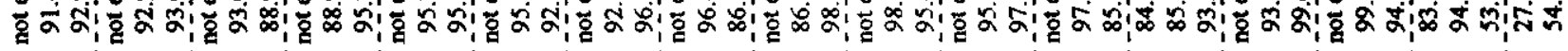

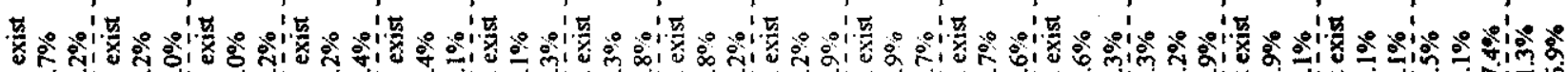

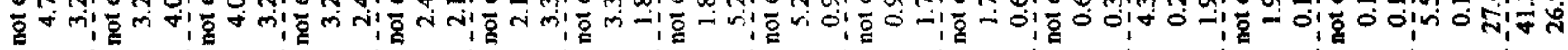

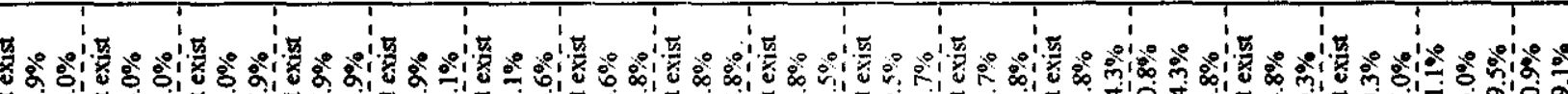

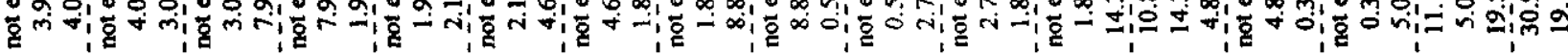

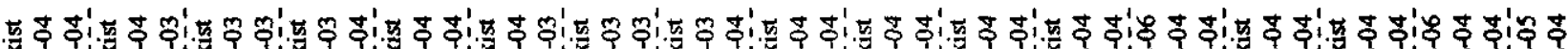

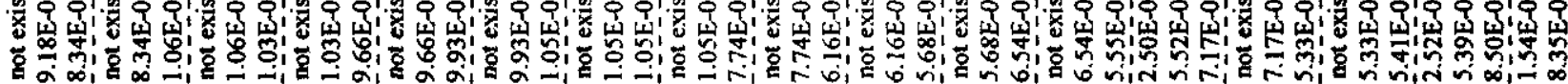

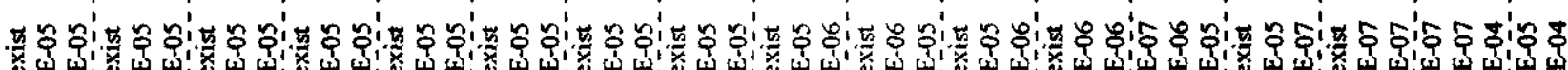

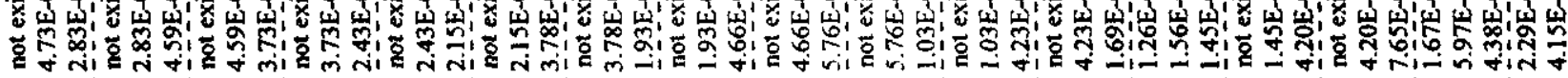

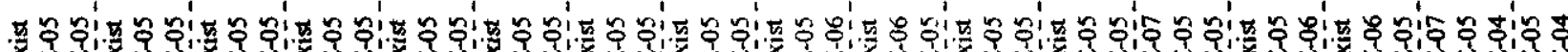

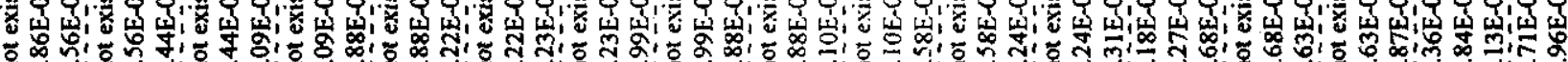
б

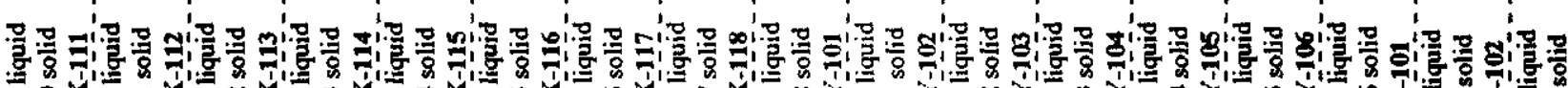
o으의.

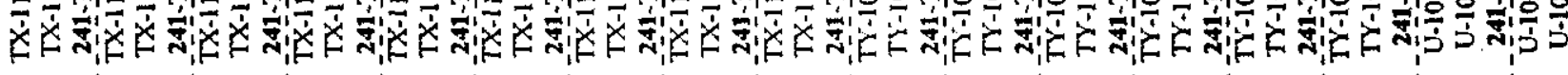




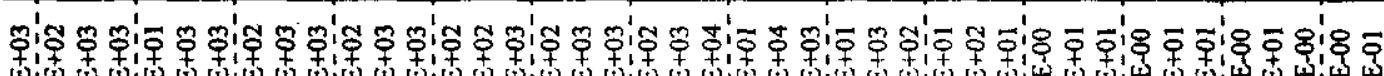

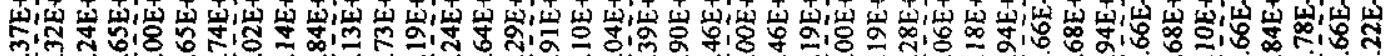
m!-

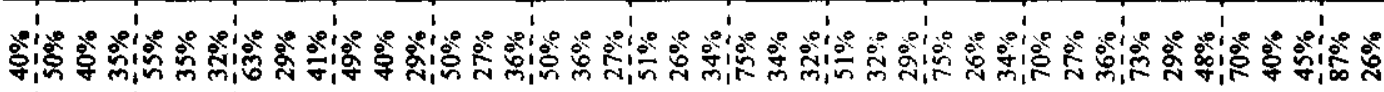
1

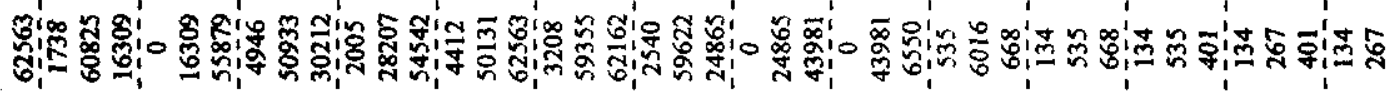
T:

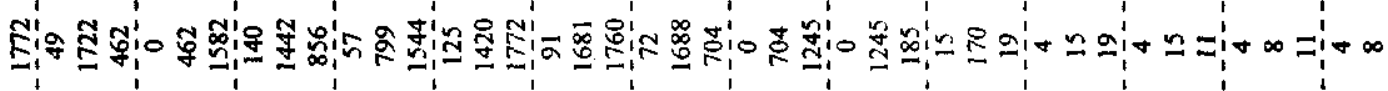

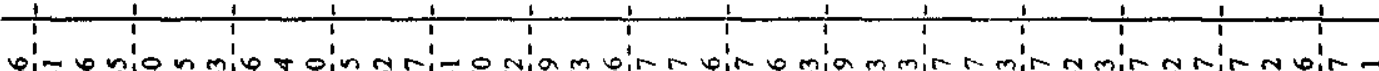

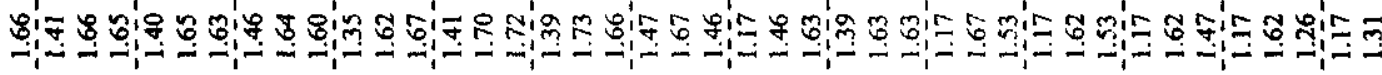

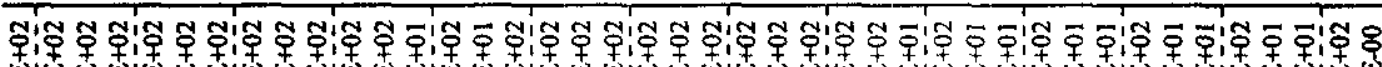

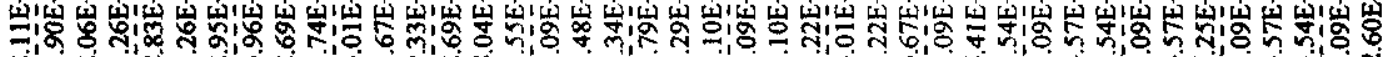

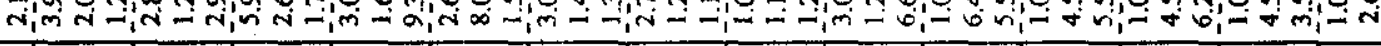

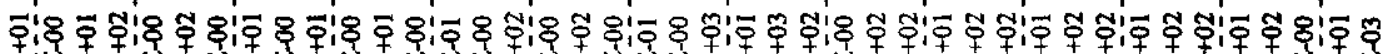

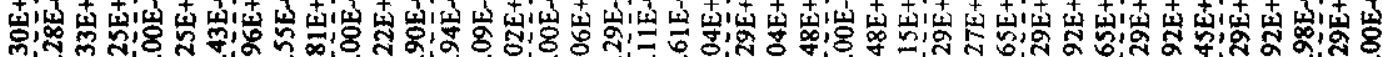

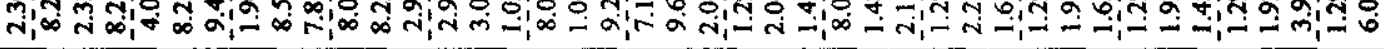
finn

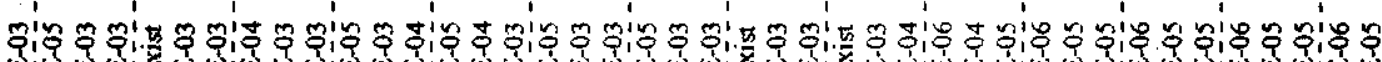

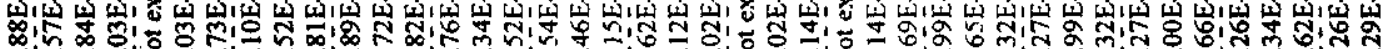

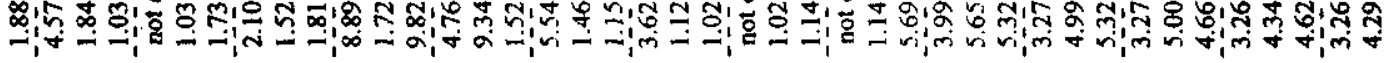

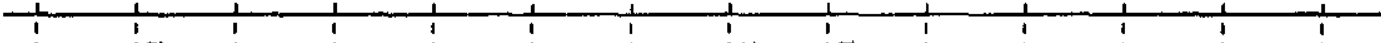

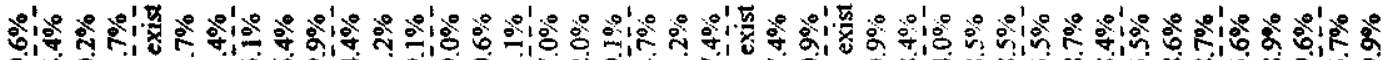

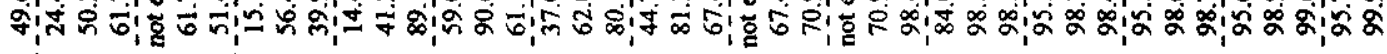

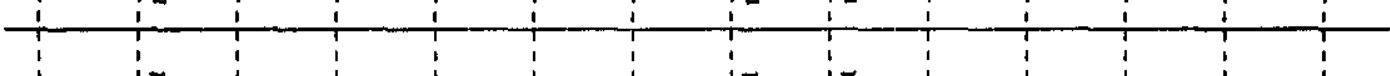

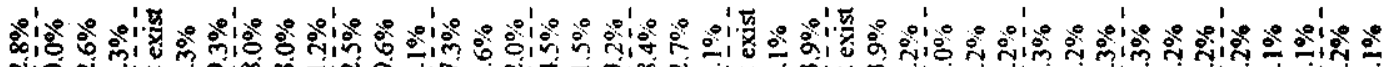

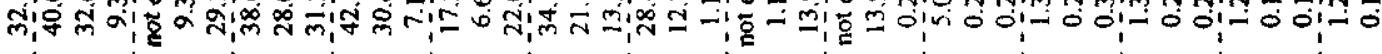
\begin{tabular}{l|l|l|l|l|l|l}
1 & \\
\hline
\end{tabular}

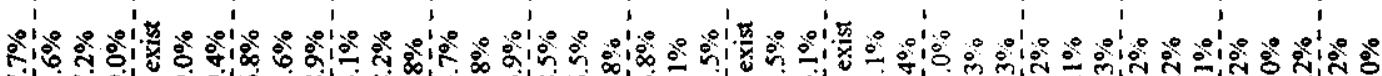

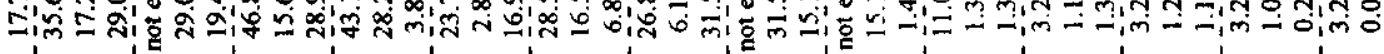

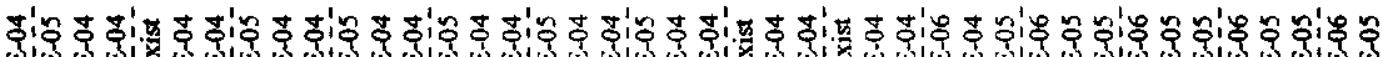

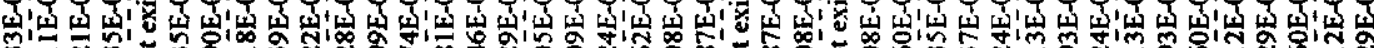

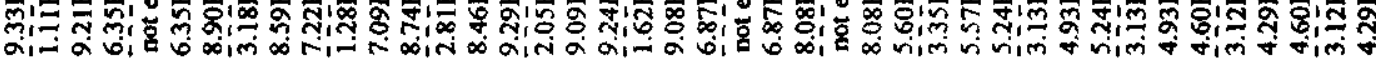

,

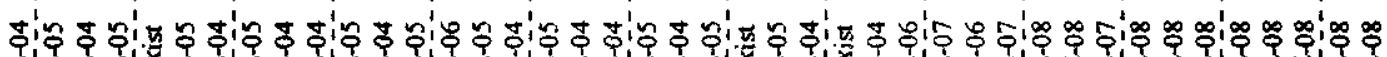

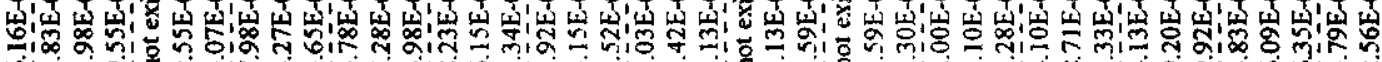
б.

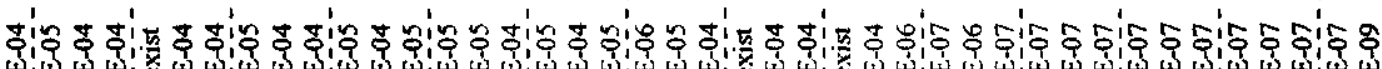

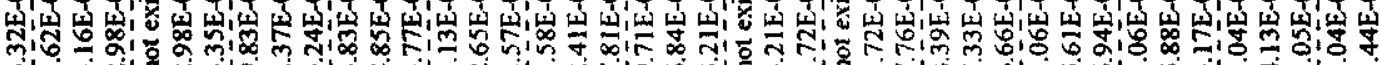
m! m

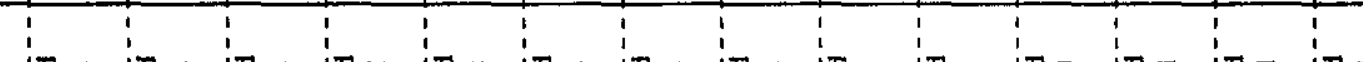

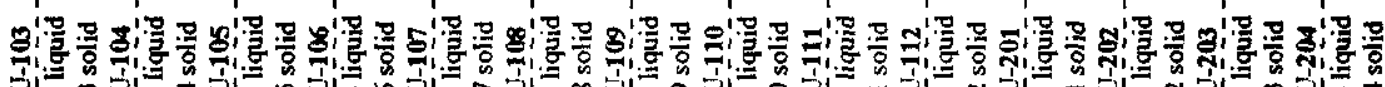

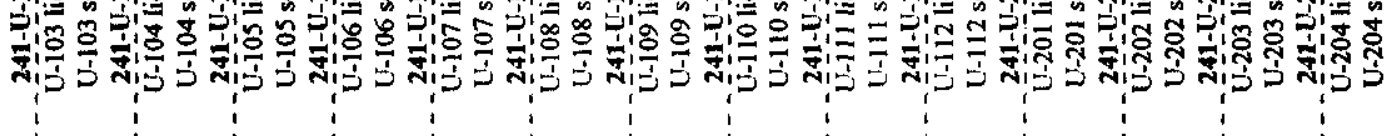


RPP-6213 REV 0

This page intentionally left blank. 


\section{B.0 POSTULATED BUMP SCENARIOS DEEMED NOT PLAUSIBLE}

\section{B.1 STEAM BUMP BY INTERSTITIAL SLUDGE SUPERHEAT}

In Section 4.0, we demonstrated the importance of a deep supernatant layer as a necessary condition for a tank bump. Here we examine the potential for a steam bump within a nonconvective layer during dryout (see Figure B-1). Steam bumps are known to occur in pure water that is rapidly brought to boiling in a vessel with smooth walls. In this case, boiling is started with large liquid water superheatings. The few bubbles that form at large superheats grow very rapidly at a rate controlled largely by heat conduction in the surrounding liquid just outside the bubbles. The process is rather unstable and could result in the ejection of liquid from the vessel (i.e., "bumping"). To prevent the occurrence of bumping, the water is usually seeded with solid particles or gas bubbles that act as nucleation sites and promote boiling at low superheats. In this case, numerous slowly growing bubbles are formed and a more uniform (smooth) boiling activity is realized. Thus, steam bumps in boiling water derive mainly from high liquid superheats. As we shall see below, significant liquid superheating is precluded in sludge by the presence of the solid phase particulate and pre-existing gas bubbles that serve as vapor-bubble nuclei.

In the analysis that follows, it is assumed that the non-convective layer evaporation process is one of bubble production by volumetric (decay) heating. This picture is believed to be conservative because water loss from the overheated layer is likely a dryout phenomenon in which an evaporation front propagates up from the bottom toward the surface of the layer. In this regard, it should be recognized that the effective upward heat flux in the sludge is very low ( $\leq 100 \mathrm{~W} \mathrm{~m}^{-2}$, see Section 3.6) and about two-to-four orders of magnitude less than typical nucleate boiling heat fluxes $\left(10^{4}\right.$ to $\left.10^{6} \mathrm{~W} \mathrm{~m}^{-2}\right)$.

Bubble growth occurs when the internal pressure (vapor pressure plus partial pressure of any additional gas) is large enough to overcome the external bubble pressure due to surface tension and yield stress $\left(2 \sigma / R+2 / \sqrt{3} \tau_{0}\right.$, where $R$ is the bubble radius, $\sigma$ is the surface tension, and $\tau_{0}$ is the yield stress in the Bingham model of the non-convective layer). The bubble is then unstable and grows at a rate governed by the inertia of the surrounding liquid and conduction heat transfer from the surrounding liquid to the bubble "surface". The initial period of inertiallylimited growth is important only in cases involving very high liquid superheats and/or low (subatmospheric) ambient pressures. This gives rise to a "waiting time" before a period of thermallydominated bubble growth. After the waiting time, which is practically non-existent for the conditions of interest here, bubbles grow by the thermally-limited process and the bubble radius after time $t$ is (see, e.g., Scriven [1959]):

$$
R=J a\left(\pi \alpha_{l} t\right)^{1 / 2}
$$

with the Jakob number, Ja, defined as

$$
J a=\frac{\rho_{\ell} c_{p, \ell}\left(T-T_{b p}\right)}{\rho_{g} h_{\ell g}}
$$


Figure B-1. Steam Bump Due to Interstitial Superheat in Non-Convective Layer (Hypothetical).

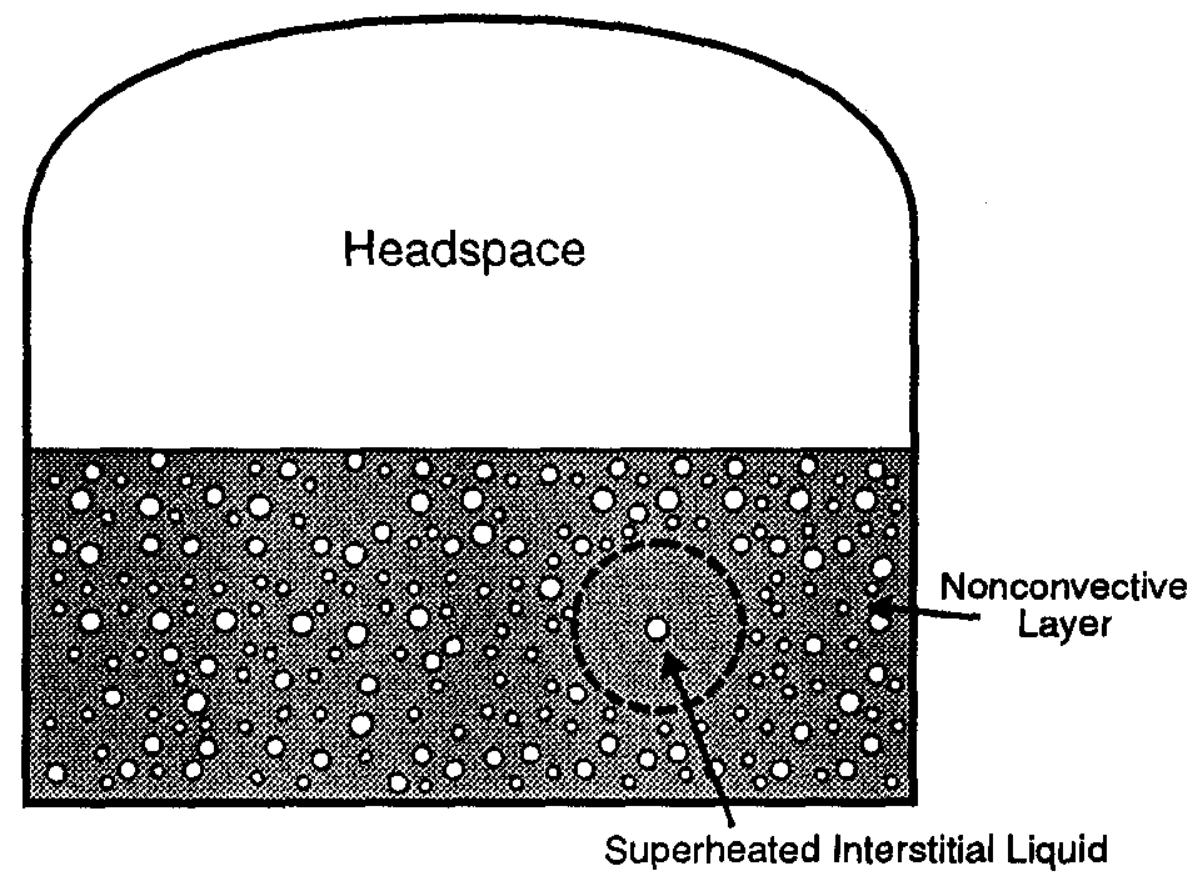

(a) Sludge with region of interstitial liquid heated above its boiling point

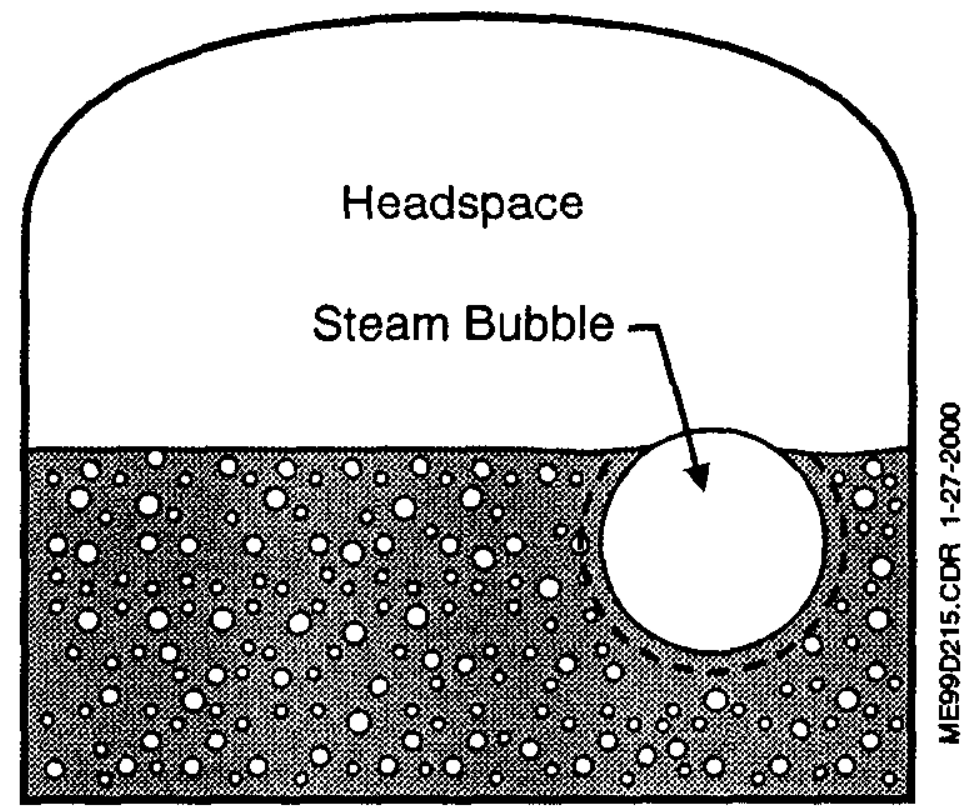

(b) Rapid bubble growth following nucleation of bubble in superheated region 
In equations (B-1) and (B-2) $\mathrm{T}_{\mathrm{bp}}, \rho_{\ell}, \mathrm{c}_{\mathrm{p}, \ell}, \alpha_{\ell}$, and $\mathrm{h}_{\ell \mathrm{g}}$ are, respectively, the boiling point, density, specific heat, thermal diffusivity, and latent heat of evaporation of the liquid (interstitial water), $T$ is the instantaneous temperature of the volumetrically heated waste, and $\rho_{\mathrm{g}}$ is the density of the vapor in the bubble. In writing equation (B-1) it is assumed that the bubble displaces both the precipitate particles and the liquid as it grows, so that the bubble only contains water vapor. If, instead, the precipitate particles are locked in place so that the bubble must push the surrounding liquid through channels in a stationary precipitate matrix, then a different bubble growth equation is required (see Epstein [1994]). For the double-shell tanks of interest, bubbles growing between stationary particles is not possible (Gauglitz et al. 1996; and Stewart et al. 1996a).

As the liquid temperature $T$ rises above the liquid boiling point owing to volumetric decay, heating new bubbles will form from the nuclei present in the liquid if the rate of energy input is higher than that removed by the bubbles nucleated previously at lower superheats. Thus, at any particular time there will be a distribution of bubble sizes within the waste. We will ignore this complication and assume that all the bubble nuclei in the system are activated as soon as the waste is heated to its boiling temperature $T_{b p}$. Subject to this assumption, the distribution of bubble sizes is a uniform one and given by $\mathrm{R}$ in equation (B-1).

By considering an energy balance for the boiling waste, we may write

$$
Q=\rho_{\ell} c_{p, \ell} \frac{d T}{d t}+N h_{\ell g} \rho_{g} 4 \pi R^{2} \frac{d R}{d t}
$$

where $\mathrm{N}$ is the number of active nuclei (precipitate + gas bubbles) per unit volume of nonconvective layer and $\mathrm{Q}$ is the volumetric decay heating rate (in $\mathrm{W} \mathrm{m}^{-3}$ ). Equation (B-3) simply states that the rate of energy input is equal to the rate of increase in liquid waste sensible heat plus the heat removed volumetrically by the growing bubbles. For small temperature increases, equation (B-3) may be integrated from $T=T_{b p}$ and $R=0$ at $t=0$ to get

$$
T-T_{b p}=\frac{Q t}{\rho_{\ell} c_{p, \ell}}-\frac{h_{\ell g} N \rho_{g}}{\rho_{\ell} c_{p, \ell}} \frac{4}{3} \pi R^{3}
$$

Eliminating $R$ between equations (B-1) and (B-4) gives

$$
\mathrm{T}-\mathrm{T}_{\mathrm{bp}}=\frac{\mathrm{Q} \mathrm{t}}{\rho_{\ell} \mathrm{c}_{\mathrm{p}, \ell}}-\frac{4 \pi \mathrm{h}_{\ell \mathrm{g}} \mathrm{N} \rho_{\mathrm{g}}}{3 \rho_{\ell} \mathrm{c}_{\mathrm{p}, \ell}}\left(\frac{\rho_{\ell} \mathrm{c}_{\mathrm{p}, \ell}}{\rho_{\mathrm{g}} \mathrm{h}_{\ell \mathrm{g}}}\right)^{3}\left(\pi \alpha_{\ell} \mathrm{t}\right)^{\frac{3}{2}}\left(\mathrm{~T}-\mathrm{T}_{\mathrm{bp}}\right)^{3}
$$

Equation (B-5) is an implicit relationship between the instantaneous liquid superheat ( $\left.T-T_{b p}\right)$ and time $(t)$. A careful examination of equation (B-5) reveals the important behavior that $T-T_{b p}$ achieves a maximum value. This value can be determined by differentiating equation (B-5) with respect to time and setting the derivatives equal to zero. To facilitate this mathematical operation, it is convenient to introduce the following dimensionless variables: 


$$
\begin{aligned}
& \tau=\left(\frac{16 \pi^{5} Q^{4} \alpha_{\ell}^{3} N^{2}}{9 \rho_{g}^{4} h_{\ell g}^{4}}\right)^{\frac{1}{7}} t \\
& \theta=\left(\frac{16 \pi^{5} k_{\ell}^{3} N^{2}}{9 Q^{3}}\right)^{\frac{1}{7}}\left(\frac{\rho_{\ell} c_{p, \ell}}{\rho_{g} h_{\ell g}}\right)^{\frac{4}{7}}\left(T-T_{b p}\right)
\end{aligned}
$$

Then, equation (B-5) reduces to the compact and universal form

$$
\theta=\tau-\tau^{\frac{3}{2}} \theta^{3}
$$

For any chosen value of dimensionless time $\tau$, the dimensionless superheat is readily seen to be the solution of a cubic equation. Equation (B-8) is plotted in Figure B-2.

To obtain the peak superheat value $\theta_{\max }$, we seek the condition $\mathrm{d} \theta / \mathrm{d} \tau=0$. From equation (B-8), we find that this condition is satisfied at all points $\left(\theta_{\max }, \tau_{\max }\right)$ that lie along the curve

$$
\frac{3}{2} \theta_{\max }^{3} \frac{\tau_{\max }^{\frac{1}{2}}}{=1.0}
$$

Finally, solving equations (B-8) and (B-9) gives the maximum dimensionless superheat

$$
\theta_{\max }=\left(\frac{4}{27}\right)^{\frac{1}{7}}=0.761
$$

which occurs at the dimensionless time

$$
\tau_{\max }=(324)^{\frac{1}{7}}=2.28
$$

Using equations (B-6) and (B-7) to convert back to physical variables, we find that the liquid superheat attains its peak value after an elapsed time

$$
t_{\max }=\left(\frac{182 \rho_{g}^{4} h_{\ell g}^{4}}{\pi^{5} Q^{4} \alpha_{\ell}^{3} N^{2}}\right)^{\frac{1}{7}}
$$

and the peak liquid superheat is 


\section{RPP-6213 REV 0}

Figure B-2. Dimensionless Liquid Superheat Versus Dimensionless Time.

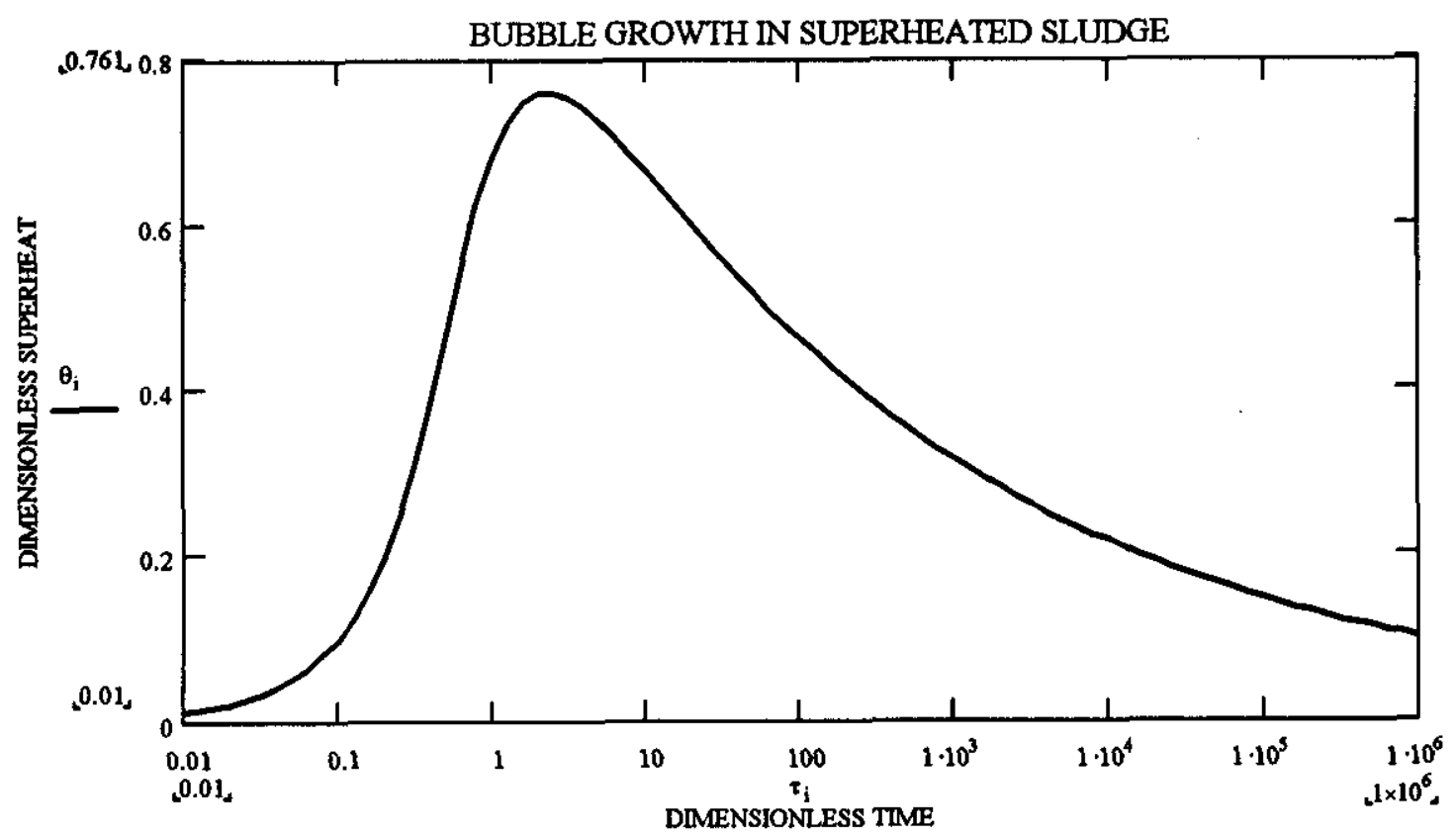




$$
T_{\max }-T_{b p}=\left(\frac{Q^{3}}{12 \pi^{5} k_{l}^{3} N^{2}}\right)^{\frac{1}{7}}\left(\frac{\rho_{g} h_{l g}}{\rho_{l} c_{p, 1}}\right)^{\frac{4}{7}}
$$

We note from equation (B-13) that the maximum liquid superheat increases with increasing decay power $\mathrm{Q}$ and decreases with increasing bubble nucleation site density $\mathrm{N}$. All the parameters in equation (B-13) are known, except for the nucleation site density $N$. The potential number density of nucleation sites in waste can be bounded from above by assuming that each precipitate particle of radius $r_{p}$ is an active site. This results in the estimate

$$
\mathrm{N}=\frac{\varepsilon_{\mathrm{s}}}{\left(\frac{4}{3}\right) \pi \mathrm{r}_{\mathrm{p}}^{3}}
$$

where $\varepsilon_{\mathrm{s}}$ is the volume fraction occupied by the solid precipitate $\left(\varepsilon_{\mathrm{s}} \approx 0.5\right)$ and $\mathrm{r}_{\mathrm{p}}$ is the effective (average) radius of the precipitate particles $\left(\mathrm{r}_{\mathrm{p}} \approx 1.0 \mu \mathrm{m}\right)$. Equation (B-14) results in $\mathrm{N} \approx 10^{17} / \mathrm{m}^{3}$, an estimate which is probably unrealistically high. The value of $\mathrm{N}$ for pre-existing gas bubbles is obtained from the relation

$$
N=\frac{\alpha}{\left(\frac{4}{3} \pi r_{b}^{3}\right)}
$$

where $\alpha$ is the volume fraction occupied by the gas bubbles $(\alpha ; 0.2)$ and $\mathfrak{r}_{\mathrm{b}}$ is the average bubble radius $\left(\mathrm{r}_{\mathrm{b}} ; 1.0 \mathrm{~mm}\right.$ ). Equation (B-15) yields $\mathrm{N} ; 5 \times 10^{7} / \mathrm{m}^{3}$ for the number density of preexisting gas-bubbles. Coincidentally, this value of $\mathrm{N}$ falls within the range of values for ordinary water (no precipitate particles) containing natural impurities (dust, etc.), namely $10^{6}$ to $10^{11} / \mathrm{m}^{3}$ (Richter 1981; Abdollahian et al. 1982; Ardon 1978; and Rivard and Travis 1980).

Some indication of the validity of equation (B-13) can be given by considering the Lipkis et al. (1956) experimental study of boiling in a volumetrically-heated aqueous $\mathrm{KOH}$ solution pool. In order to prevent bumpiness, a sheet of teflon ${ }^{T M}$ with embedded aluminum chips was placed on the bottom of the test vessel. The aluminum $/ \mathrm{KOH}$ reaction produced very many tiny hydrogen bubbles which acted as vapor-bubble nuclei. The volumetric power applied to the pool was nominally $1.5 \times 10^{7} \mathrm{~W} \mathrm{~m}^{-3}$. The corresponding measured superheat was $\Delta \mathrm{T}=0.5^{\circ} \mathrm{C}$. Substituting these measured values into equation (B-13), together with the water physical properties $\mathrm{h}_{\mathrm{lg}}=2.3 \times 10^{6} \mathrm{~J} \mathrm{~kg}^{-1}, \mathrm{k}_{\mathrm{f}}=0.68 \mathrm{~W} \mathrm{~m}^{-1} \mathrm{~K}^{-1}, \mathrm{c}_{\mathrm{p}, \ell}=4200 \mathrm{~J} \mathrm{~kg}^{-1} \mathrm{~K}^{-1}, \rho_{\mathrm{g}}=0.6 \mathrm{~kg} \mathrm{~m}^{-3}$, and $\rho_{\ell}=960 \mathrm{~kg} \mathrm{~m}^{-3}$, and solving for $\mathrm{N}$ gives

$$
\mathrm{N}=2.3 \times 10^{9} / \mathrm{m}^{3}
$$

This is an encouraging result because it falls in the middle of the expected range of site densities $10^{6}$ to $10^{11} / \mathrm{m}^{3}$. 
Inserting our previous estimate $\mathrm{N} ; 5 \times 10^{7} / \mathrm{m}^{3}$ for $1.0 \mathrm{~mm}$ pre-existing gas bubbles together with the decay heat rate $\mathrm{Q}=200 \mathrm{~W} \mathrm{~m}^{-3}$ into equation (B-13) gives a maximum waste-liquid superheat of only $T_{\max }-T_{b p}=1.2 \times 10^{-2}{ }^{\circ} \mathrm{C}$. Even if one adopts the lower end of the site density range for gas bubble, namely $\mathrm{N}=4 \times 10^{5} / \mathrm{m}^{3}$ for $\mathrm{r}_{\mathrm{b}}=5 \mathrm{~mm}$, the liquid superheat in boiling waste is still small and equal to $T_{\max }-\mathrm{T}_{\mathrm{bp}}=4.8 \times 10^{-2}{ }^{\circ} \mathrm{C}$. Laboratory experience with boiling liquids indicate that these superheats are far too small to produce bumpy boiling action. For example, smooth volumetric boiling activity was achieved in the experiments of Lipkis et al. (1956) with measured water superheats as high as $0.5^{\circ} \mathrm{C}$. We conclude by stating that the volume-heating rate in the non-convective layer is too low to produce a steam bump.

\section{B.2 STEAM BUMP BY LOCAL CONVECTION IN SLUDGE (FUMAROLES)}

Tank bumps have been postulated to occur via a buoyant displacement within a hot, otherwise non-convective layer (see Figure B-3). The mechanism that might cause a buoyant displacement within the non-convective layer is as follows. Generally, the waste material near the mid-plane of the non-convective layer retains more gas than the overlying layer that extends to the surface. A Taylor instability develops in which the central waste material is presumed buoyant enough with respect to the overlying layer to rise and mix with the overlying layer. During an overheated condition, the temperature of the sludge near the mid-plane of the non-convective layer is close to the boiling point of the interstitial liquid. As the initially, centrally located hot sludge rises through the non-convective layer, it experiences a reduction in hydrostatic pressure and its interstitial liquid may boil and produce steam. We emphasize that this mechanism differs from the rollover mechanism for steam production in that an overlying convective layer is not required - the buoyant displacement is postulated to occur within the non-convective layer itself. However, in the absence of a convective layer, it is difficult to envision significant waste release from the tank.

In order to rigorously apply the Taylor instability theory to Hanford sludge, it is necessary to take into account the viscoplastic nature of the sludge material. Construction of the equations and their solution for this case would be very difficult and no analysis of this problem is available. Here, we content ourselves with an analysis that considers the waste material to be a viscous Newtonian material. That is, the waste gel is assumed to be shocked into a liquefied state. The same waste rheology was assumed by Meyer et al. (1997) in their Taylor-instability model of buoyant displacements in DSTs. We are concerned with the waste tank situation in which a central horizontal waste layer of thickness $h$ and low density lies below a relatively thick, dense layer that extends to the surface of the waste. The lower layer is less dense than the upper layer owing to a higher degree of gas retention in the lower layer. The two layers are assumed to have the same kinematic viscosity.

An approximate dispersion relation for the problem described above has been derived by Plesset and Whipple (1974) and is 
Figure B-3. Steam Bump Due to Local Convection in Non-Convective Layer (Hypothetical, "Fumarole").

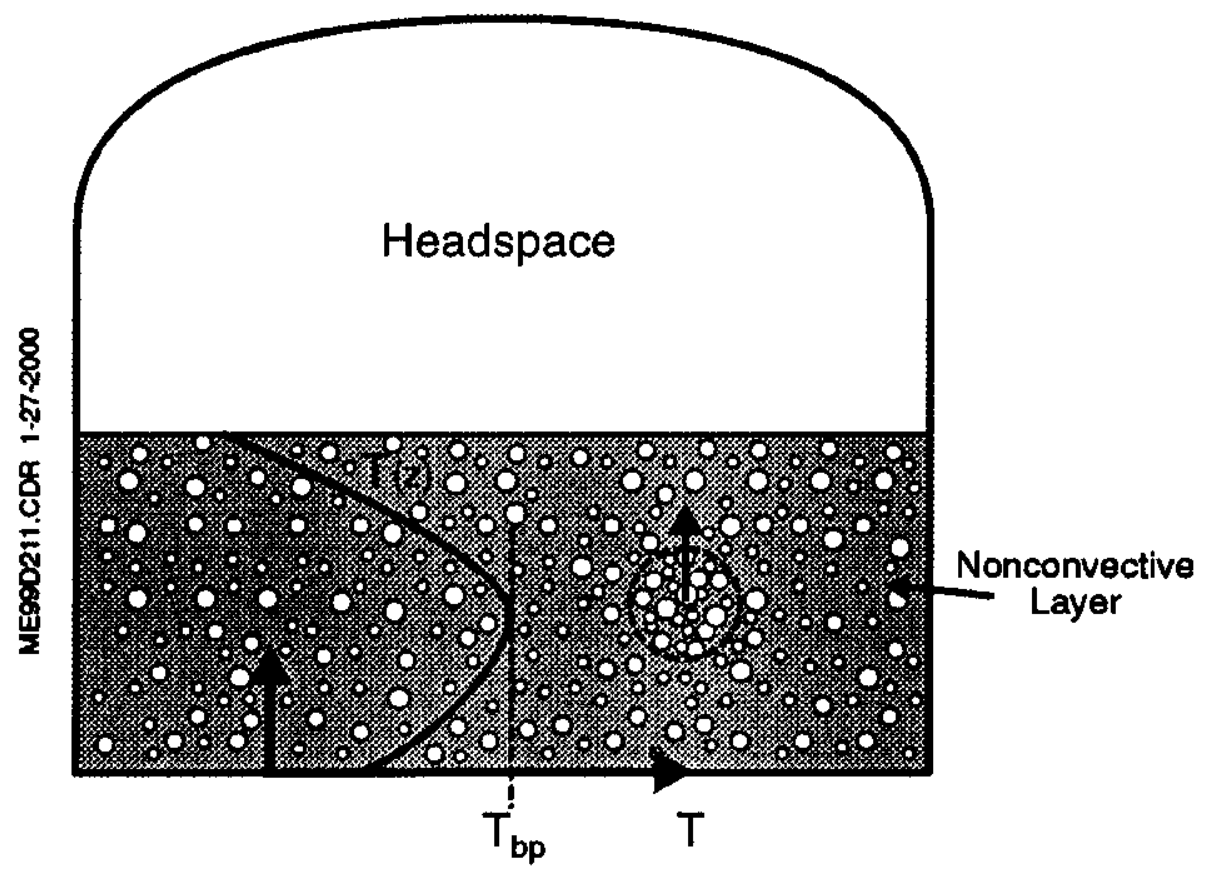

(a) Hot sludge parcel begins to rise and superheat.

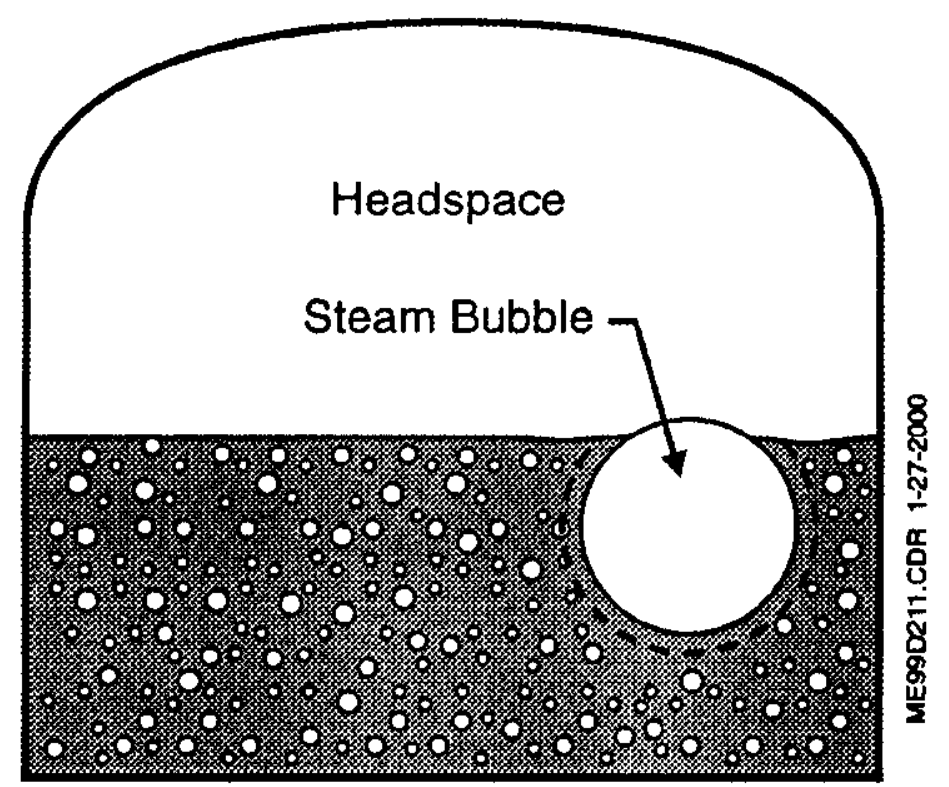

(b) Steam bubble growth within hot sludge parcel 


$$
h=v k^{2}\left[1+\frac{g k \Delta \rho / \rho}{[1+\operatorname{coth}(k h)]\left(v k^{2}\right)^{2}}\right]^{1 / 2}-v k^{2}
$$

where $v$ and $\rho$ are the kinematic viscosity and the density of the waste material, respectively, $\Delta \rho$ is the density difference between the upper and lower layers, $\mathrm{g}$ is the gravitational constant, $\mathrm{k}$ is the wave number, and $n$ is the growth rate of the unstable waves that develop at the boundary that separates the two layers. The wave number is related to the disturbance wavelength by the definition

$$
\mathbf{k}=\frac{2 \pi}{\lambda}
$$

and the characteristic time for the waves to grow to an amplitude of the order of the wavelength is

$$
\tau \quad \square \frac{1}{n}
$$

A typical in situ measurement in DSTs shows a void fraction increment in the lower layer over the upper layer of $10 \%$ (Stewart et al. 1996b). The void fraction in a hypothetical tank without a supernate layer could be much higher than $10 \%$. Assuming $30 \%$ for the void fraction, $\Delta \rho / \rho ; 0.3$. The waste viscosity $\mu$ is assigned the value $10^{4} \mathrm{~Pa}$ s which is the peak measured value at the bottom of undisturbed DST waste, that is first-pass ball rheometer measurements reported by Stewart et al. (1996b) and Stewart (1998). The waste material density is approximately $\rho ; 1600 \mathrm{~kg} \mathrm{~m}^{-3}$ so that $v=6.25 \mathrm{~m}^{2} \mathrm{~s}^{-1}$. Figure B-4 shows the characteristic time for the buoyant displacement to develop as a function of the wavelength of the disturbance that caused the instability for several relevant values of the thickness $h$ of the central layer. The minimums in the curves correspond to the most unstable disturbance wavelengths. These waves cannot materialize within the Hanford waste tanks because they exceed the lateral dimensions of the tanks $(\sim 20 \mathrm{~m})$. However, smaller wavelengths are also unstable and may occur with lower probability. The most probable wavelength coincides with the tank diameter. Despite the high viscosity assigned to the material, seven orders of magnitude larger than the viscosity of water, these waves will develop and reach the surface of the waste less than about $10 \mathrm{~s}$.

Obviously, the Taylor instability has the potential of producing significant quantities of steam by transporting massive quantities of waste material from the hot mid-plane region to the surface in a relatively short period of time compared with, say, bubble rise times in the assumed Newtonian waste layer. When the sludge temperature is low and not conducive to steam production, the Taylor instability should still be operative and result in very efficient and dangerous gas release events. However, this has not been observed in waste tanks that lack a convective layer (e.g., SSTs). Field measurements indicate gas release times of at least 1.0 hour in duration. The release efficiencies are less than $10 \%$ with most of the releases in the 0.1 to $1.0 \%$ range. The actual small, lethargic releases are consistent with a mechanism of gas percolation to the surface through bubble inter-connectivity. Apparently, the strength of the material, which enables it to 


\section{RPP-6213 REV 0}

Figure B-4. Wave Growth Time Versus Disturbance Wave Length; Depth of Less-Dense Layer as a Parameter. $\left(\Delta \rho / \rho=0.3, \mu / \rho=6.3 \mathrm{Kg} \mathrm{m}^{-3}\right)$

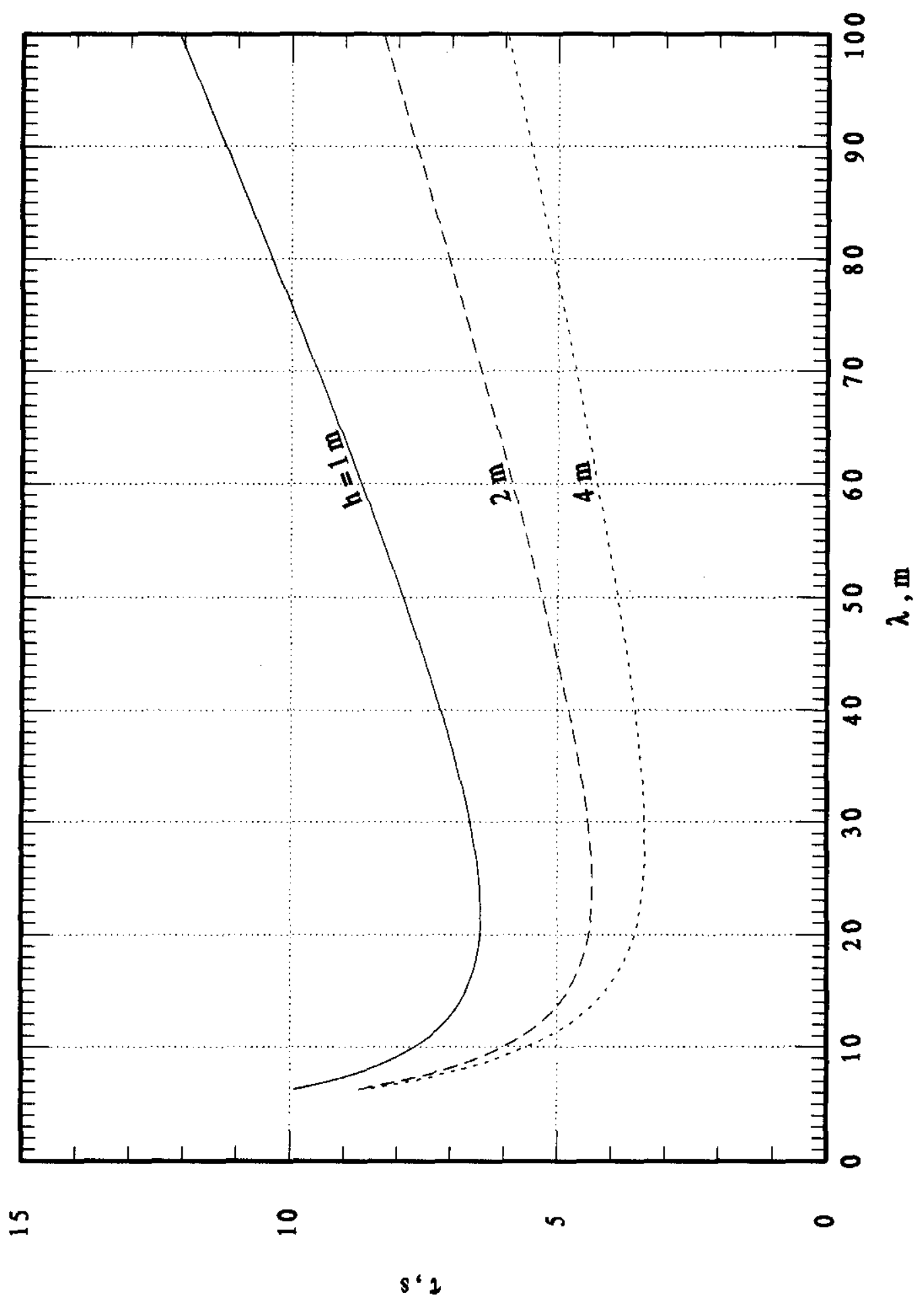


accumulate gas, stabilizes the waste layer against an internal buoyant displacement. If the Taylor stability analysis were performed for the viscoplastic case (a difficult analysis), one would most likely find a cutoff wavelength that exceeds the tank diameter.

\section{B.3 REFERENCES}

Abdollahian, D., Healzer, J., Janssen, E., and Amos, C., 1982, Critical Flow Data Review and Analysis, EPRI NP-2192.

Ardon, K. H., 1978, A Two-Fluid Model for Critical Vapor-Liquid Flow, Int. J. Multiphase Flow, Vol. 4, pp. 323-333.

Epstein, M., 1994, A Similarity Solution for Combined Hydrodynamic and Heat Transfer Controlled Growth in a Porous Medium, J. Heat Transfer, Vol. 116, pp. 516-518.

Gauglitz, P. A., Rassat, S. D., Bredt, P. R., Konynenbelt, J. H., Tingey, S. M., and Mendoza, D. P., 1996, Mechanisms of Gas Bubble Retention and Release: Results for Hanford Waste Tanks 241-S-102 and 241-SY-103 and Single-Shell Tank Simulants, Pacific Northwest National Laboratory Report PNNL-11298, Richland, WA.

Lipkis, R. P., Liu, C., and Zuber, N., 1956, Measurement and Prediction of Density Transients in a Volume-Heated Boiling System, Chem. Engng. Progress Symposium, Series 52, pp. 105-113.

Meyer, P. A., Brewster, M. E., Bryan, S. A., Chen, G., Pederson, L. R., Stewart, C. W., and Terrones, G., 1997, Gas Retention and Release Behavior in Hanford Double-Shell Waste Tanks, PNNL-11536, Rev. 1, Pacific Northwest National Laboratory, Richland, WA.

Plesset, M. S., and Whipple, C. G., 1974, Viscous Effects in Rayleigh-Taylor Instability, Physics of Fluids, Vol. 17, pp. 1-7.

Richter, H. J., 1981, Separated Two-Phase Flow Model: Application to Critical Two-Phase Flow, EPRI NP-1800.

Rivard, W. C., and Travis, J. R., 1980, A Nonequilibrium Vapor Production Model for Critical Flow, Nuclear Sci. and Engng., Vol. 74, pp. 40-48.

Scriven, L. A., 1959, On the Dynamics of Phase Growth, Chem. Eng. Sci., Vol. 10, pp. 1-13.

Stewart, C. W., Brewster, M. E., Gauglitz, P. A., Mahoney, L. A., Meyer, P. A., Recknagle, K. P., and Reid, H. C., 1996a, Gas Retention and Release Behavior in Hanford Single-Shell Waste Tanks, PNNL-1 1391, Pacific Northwest National Laboratory, Richland, WA.

Stewart, C. W., Alzheimer, J. M., Brewster, M. E., Chen, G., Mendoza, R. E., Reid, H. C., Shepard, C. L., and Terrones, G., 1996b, In Situ Rheology and Gas Volume in Hanford 
RPP-6213 REV 0

Double-Shell Waste Tanks, Pacific Northwest National Laboratory Report PNNL-11296, Richland, WA.

Stewart, C. W., 1998, Presentations, Presented at Stage II Experts' Panel Elicitation Workshop \#1, Richland, WA. 


\section{C.0 RISE DISTANCE TO YIELDING OF VOID-BEARING PARCEL}

In this appendix the assumption that the buoyant displacement parcel becomes fluid after rising only a short distance $z$ is examined. Meyer et al. (1997) presented a model that describes the energy requirements to yield the gas-retaining material and transform it into a liquid state. According to the model, the material within the buoyant parcel will yield and start to flow when the available buoyant energy,

$$
E_{b}=\int_{0}^{z} g\left[\rho_{\ell} V(z)-\rho_{s} V_{s}\right] d z
$$

equals or exceeds the energy required to yield the parcel,

$$
E_{y}=V_{0} \varepsilon_{y} \tau_{y}\left(1-\alpha_{0}\right)
$$

In the above equations $\rho_{\ell}$ is the density of the convective layer, $g$ is the gravitational constant, $\varepsilon_{y}$ is the strain at failure $(; 1.0), \tau_{y}$ is the yield stress of the non-convective layer material, $\alpha_{0}$ is the initial void fraction within the non-convective layer which should be nearly equal to the neutral buoyancy void fraction $\left(\alpha_{N B}\right), V_{0}$ and $V(z)$ are, respectively, the initial volume of the parcel and the volume of the parcel at elevation $z$ above the mid-plane of the non-convective layer, and $\rho_{s}$ and $V_{s}$ are, respectively, the density and volume of the void-free material within the non-convective layer.

The ratio of the two energies given by equations $(\mathrm{C}-1)$ and $(\mathrm{C}-2)$ is

$$
\frac{E_{b}}{E_{y}}=\frac{\int_{0}^{z} g\left[\rho_{\ell} V(z)-\rho_{s} V_{s}\right] d z}{V_{0} \varepsilon_{y} \tau_{y}\left(1-\alpha_{0}\right)}
$$

Meyer et al. (1997) compared equation (C-3) with scaled experimental results (Stewart et al. 1996) and concluded that most of the buoyant parcel will yield when $\mathrm{E}_{\mathrm{b}} / \mathrm{E}_{\mathrm{y}}>$ 5.0. They concluded that for current tank conditions the buoyant parcel must rise several meters before the buoyant parcel yields and flows. It should be recognized, however, that the increase in buoyant energy of the parcel at the current, relatively low waste temperatures is due mainly to bubble growth via hydrostatic pressure reduction with distance $z$. As the temperature of the non-convective layer increases and approaches the boiling point of the layer, bubble expansion by evaporation becomes increasingly important and the Meyer et al. (1997) criterion must be modified to account for this.

Assuming that the steam/non-condensible gas mixture that fills the void is always in equilibrium with the interstitial liquid at temperature $T_{N C}$ (see equation (4-12) with $P_{v}=P_{e q}$ ), we have

$$
\frac{V_{b}(z)}{V_{b}(0)}=\frac{P_{h s}+\rho_{\ell} g H-P_{e q}\left(T_{N C L}\right)}{P_{h s}+\rho_{\ell} g(H-z)-P_{e q}\left(T_{N C L}\right)}
$$


where $V_{b}(0)$ is the initial void volume in the buoyant parcel before its ascent (i.e., at $z=0$ ), $V_{b}(z)$ is the void volume at elevation $z$, and $H$ is the distance from the mid-plane of the nonconvective layer to the surface of the waste. The equilibrium assumption underlying equation (C-4) is justified because it gives results in good agreement with the non-equilibrium model when the temperature is low (see Figure 4-3) and when the temperature is high because the rise distances to yielding are small and the rise velocities through these distances are low. Note that in the low-temperature limit $\mathrm{P}_{\mathrm{eq}}\left(\mathrm{T}_{\mathrm{NCL}}\right) \ll<\mathrm{P}_{\text {hs }}$ and equation (C-4) reduces to the void expansion equation used by Meyer et al. (1997).

The total volume of the parcel at elevation $z$ is

$$
V(z)=V_{s}+V_{b}(z)
$$

The density of the void-free material is related to $\rho_{\ell}$ and the neutral buoyancy void fraction $\alpha_{\mathrm{NB}}$ by

$$
\rho_{\mathrm{s}}=\frac{\rho_{\ell}}{1-\alpha_{\mathrm{NB}}}
$$

The volume of the void-free material and the initial void volume in the buoyant parcel may be expressed as

$$
\begin{aligned}
& \mathrm{V}_{\mathrm{s}}=\mathrm{V}_{0}\left(1-\alpha_{0}\right) \\
& \mathrm{V}_{\mathrm{b}}(0)=\alpha_{0} \mathrm{~V}_{0}
\end{aligned}
$$

Combining equations (C-1) and (C-5) to equation (C-8) gives

$$
E_{b}=g \rho_{\ell} \alpha_{0} V_{0} \int_{0}^{z}\left[\frac{V_{b}(z)}{V_{b}(0)}-k\right] d z
$$

where $k=\frac{\alpha_{\mathrm{NB}}\left(1-\alpha_{0}\right)}{\alpha_{0}\left(1-\alpha_{\mathrm{NB}}\right)}$

Substituting equation (C-4) into equation (C-9) and carrying out the indicated integration yields

$$
E_{b}=-g \rho_{\ell} \alpha_{0} V_{0} H\left[\frac{1+\gamma}{\gamma} \ln \left(1-\frac{\gamma}{1+\gamma} \frac{z}{H}\right)+k \frac{z}{H}\right]
$$

where $\gamma=\frac{\rho_{\ell} \mathrm{gH}}{\mathrm{P}_{\mathrm{hs}}-\mathrm{P}_{\mathrm{eq}}\left(\mathrm{T}_{\mathrm{NCL}}\right)}$

Dividing equation (C-11) by equation (C-2) leads to the criterion for the yielding of the parcel: 


$$
\frac{E_{b} \varepsilon_{y} \tau_{y}\left(1-\alpha_{0}\right)}{E_{y} g \rho_{\ell} \alpha_{0} H}=-\frac{1+\gamma}{\gamma} \ln \left(1-\frac{\gamma}{1+\gamma} \frac{z}{H}\right)-k \frac{z}{H}
$$

which is an implicit expression for the rise distance $z$ where failure of the parcel occurs. If the initial void fraction $\alpha_{0}$ is equal to the neutral buoyancy void fraction, then $k=1$ in equation (C-13).

Before solving equation (C-13), it is important to point out that the equation becomes invalid as $\mathrm{T}_{\mathrm{NCL}}$ approaches the boiling point $\mathrm{T}_{\mathrm{bp}}$ of the non-convective layer at the mid-plane static pressure, as defined by the equation

$$
P_{e q}\left(T_{b p}\right)=P_{h s}+\rho_{\ell} g H=P_{N C L}
$$

This is because as $\mathrm{T}_{\mathrm{bp}}$ is approached equation (C-4) predicts more vapor production than is thermodynamically possible by converting the available sensible energy in the buoyant parcel to steam. Indeed the minimum rise distance $z_{\min }$ to yielding the parcel may be determined by replacing equation (C-4) with a void growth or "flashing" expression that accounts for the finite thermal energy stored in the void-free non-convective layer material.

If the sensible heat available in the buoyant parcel is adiabatically delivered to the surfaces of the bubbles, the mass $m_{v}$ of steam produced as the parcel rises to elevation $z$ is

$$
m_{v}(z)=m_{v}(0)+\frac{m_{s} c_{s}\left[T_{N C L}-T(z)\right]}{h_{f v}}
$$

where $m_{v}(0)$ is the initial mass of steam in the parcel, $c_{s}$ and $m_{s}$ are, respectively, the specific heat and mass of the void-free material, $h_{\mathrm{fv}}$ is the latent heat of evaporation of the parcel's interstitial liquid, and $T(z)$ is the temperature of the parcel at elevation $z$. Using the ideal gas law, $m_{v}(z)$ and $m_{v}(0)$ can be expressed as

$$
\begin{aligned}
& m_{v}(z)=\frac{P(z) V_{b}(z)}{R T(z)} \\
& m_{v}(0)=\frac{P_{N C L} V_{b}(0)}{R T_{N C L}}
\end{aligned}
$$

where $\mathbf{R}$ is the ideal gas constant for water, $\mathrm{P}(\mathrm{z})$ is the static pressure at elevation $z$, and $\mathbf{P}_{\mathrm{NCL}}$ is the pressure in the non-convective layer (see equation $(\mathrm{C}-14)$ ). Also, $\mathrm{m}_{\mathrm{s}}$ is given by

$$
\mathrm{m}_{\mathrm{s}}=\rho_{\mathrm{s}} \mathrm{V}_{\mathrm{s}}=\rho_{\mathrm{s}} \mathrm{V}_{0}\left(1-\alpha_{0}\right)
$$

where again $\rho_{s}$ is the density of the void-free non-convective material. 
Substituting equations (C-16) to (C-18) into equation (C-15) results in

$$
V_{b}(z)=\frac{T(z)}{T_{N C L}} \frac{P_{N C L}}{P(z)} V_{b}(0)+\frac{R T(z) \rho_{s} V_{0}\left(1-\alpha_{0}\right) c_{s}}{h_{f v} P(z)}\left[T_{N C L}-T(z)\right]
$$

It will be seen below that the parcel rises only a short distance before it yields; consequently it is permissible to exploit the following approximations in equation (C-19):

$$
\begin{array}{lll}
\mathrm{T}(\mathrm{z}) & \square & \mathrm{T}_{\mathrm{NCL}} \\
\mathbf{P}(\mathbf{z}) & \square & \mathbf{P}_{\mathrm{NCL}}
\end{array}
$$

except, of course, for the temperature difference term $T_{\mathrm{NCL}}-\mathrm{T}(\mathrm{z})$. This term may be approximated by the linearized form of the Clausius-Clapeyron equation

$$
T_{N C L}-T(z)=\frac{T_{N C L}^{2} R}{h_{f v}}\left[\frac{P_{N C L}-P(z)}{P_{N C L}}\right]=\frac{T_{N C L}^{2} R}{h_{f v}}\left[1-\frac{P_{h s}+\rho_{\ell} g(H-z)}{P_{h s}+\rho_{\ell} H}\right]
$$

Combining equations (C-19) to (C-22) gives the desired expression for the void volume enhancement in the rising and "flashing" buoyant parcel:

$$
\frac{V_{b}(z)}{V_{b}(0)}=1+\frac{J \rho_{\ell} g z}{P_{N C L}}
$$

where $J=\frac{\mathrm{R}^{2} \mathrm{~T}_{\mathrm{NCL}}^{3} \rho_{\mathrm{s}}\left(1-\alpha_{0}\right) \mathrm{c}_{\mathrm{s}}}{\mathrm{h}_{\mathrm{fv}}^{2} \mathrm{P}_{\mathrm{NCL}} \alpha_{0}}$

Inserting equation (C-23) into equation (C-9) yields

$$
\mathrm{E}_{\mathrm{b}}=\mathrm{g} \rho_{\ell} \alpha_{0} \mathrm{~V}_{0}\left[\frac{\mathrm{J} \rho_{\ell} g \mathrm{z}^{2}}{2 \mathrm{P}_{\mathrm{NCL}}}+(1-\mathrm{k}) \mathrm{z}\right]
$$

Forming the ratio $E_{b} / E_{y}$ and solving the result for $z$ in the practical limit $k=1$ gives the following expression for the minimum rise distance to yielding of the buoyant parcel:

$$
z_{\min }=\left[\frac{2 \mathrm{E}_{\mathrm{b}} \mathrm{P}_{\mathrm{NCL}} \varepsilon_{\mathrm{y}} \tau_{\mathrm{y}}\left(1-\alpha_{0}\right)}{\mathrm{E}_{\mathrm{y}} \mathrm{g}^{2} \rho_{\ell}^{2} \alpha_{0} \mathrm{~J}}\right]^{1 / 2}
$$

The solution to equation (C-13) for parameter values that correspond to conditions in tank 241-AZ-102 is presented in Figure C-1. The parameter values used in the calculations are listed in Table C-1. At low non-convective layer temperatures the parcel maintains a yield stress during its first 2 meters of travel. However, the vertical distance required to turn the parcel into a 
RPP-6213 REV 0

Figure C-1. Rise Distance to Yielding of Void-Bearing Parcel Versus Temperature of NonConvective Layer; Input Parameters Pertain to Tank 241-AZ-102.

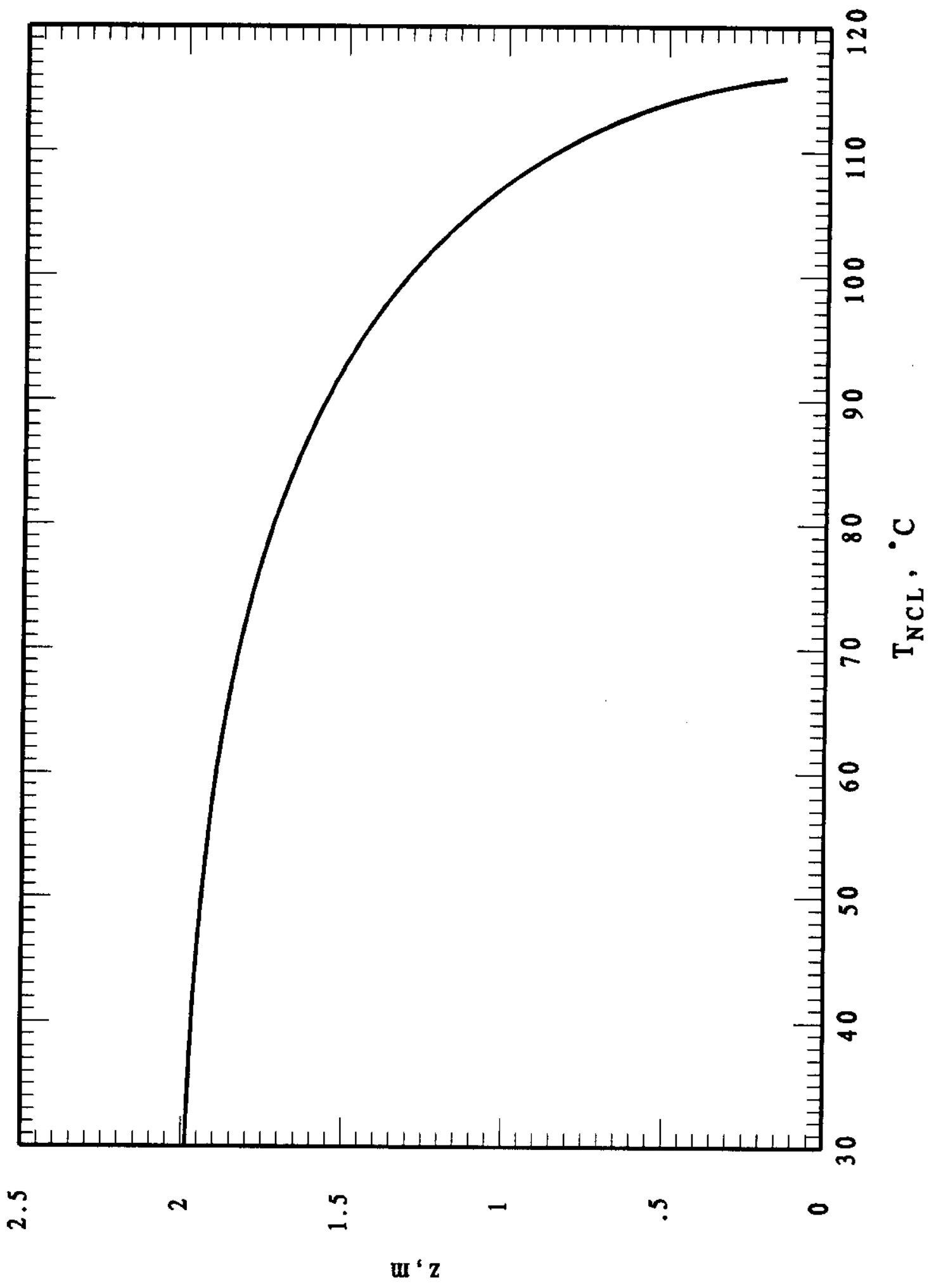

FAI/00-14, Rev. 0

C-5

May 2000 
Table C-1. Parameter Values for the Buoyant Parcel Yielding Criterion (Tank 241-AZ-102).

\begin{tabular}{|l|l|}
\hline$\alpha_{0}=\alpha_{\mathrm{NB}}=0.262$ & Initial void fraction. \\
\hline $\mathrm{H}=7.44 \mathrm{~m}$ & Depth to mid-plane of non-convective layer. \\
\hline$\rho_{l}=1100 \mathrm{~kg} \mathrm{~m}^{-3}$ & Density of supernatant. \\
\hline$\rho_{\mathrm{s}}=1490 \mathrm{~kg} \mathrm{~m}^{-3}$ & Density of void-free non-convective layer material. \\
\hline$c_{\mathrm{s}}=4200 \mathrm{~kg} \mathrm{~m}^{-3}$ & Specific heat of void-free non-convective layer material. \\
\hline$\tau_{\mathrm{y}}=100 \mathrm{~Pa}$ & Yield strength of non-convective layer material. \\
\hline$\varepsilon_{\mathrm{y}}=1.0$ & Strain of non-convective layer material at yielding. \\
\hline $\mathrm{E}_{\mathrm{b}} / \mathrm{E}_{\mathrm{y}}=5.0$ & Buoyant-to-yield energy ratio. \\
\hline $\mathrm{h}_{\mathrm{fv}}=2.2 \times 10^{6} \mathrm{~J} \mathrm{~kg}^{-1}$ & Latent heat of evaporation of interstitial water. \\
\hline
\end{tabular}

fluid decreases rapidly as its boiling point is approached. For all practical purposes, the boiling or near-boiling parcel can be assumed to liquefy as soon as it begins to move $\left(z_{\min }=13 \mathrm{~cm}\right)$.

\section{References}

Meyer, P. A., Brewster, M. E., Bryan, S. A., Chen, G., Pederson, L. R., Stewart, C. W., and Terrones, G., 1997, Gas Retention and Release Behavior in Hanford Double-Shell Waste Tanks, PNNL-11536, Rev. 1, Pacific Northwest National Laboratory, Richland, WA.

Stewart, C. W., Brewster, M. E., Gauglitz, P. A., Mahoney, L. A., Meyer, P. A., Recknagle, K. P., and Reid, H. C., 1996, Gas Retention and Release Behavior in Hanford Single-Shell Waste Tanks, PNNL-11391, Pacific Northwest National Laboratory, Richland, WA. 


\section{D.0 BUBBLE GROWTH MODEL AND INPUT}

\section{D.1 GASCAVGRTH.FOR: Calculations for Chapter 4 Figures}

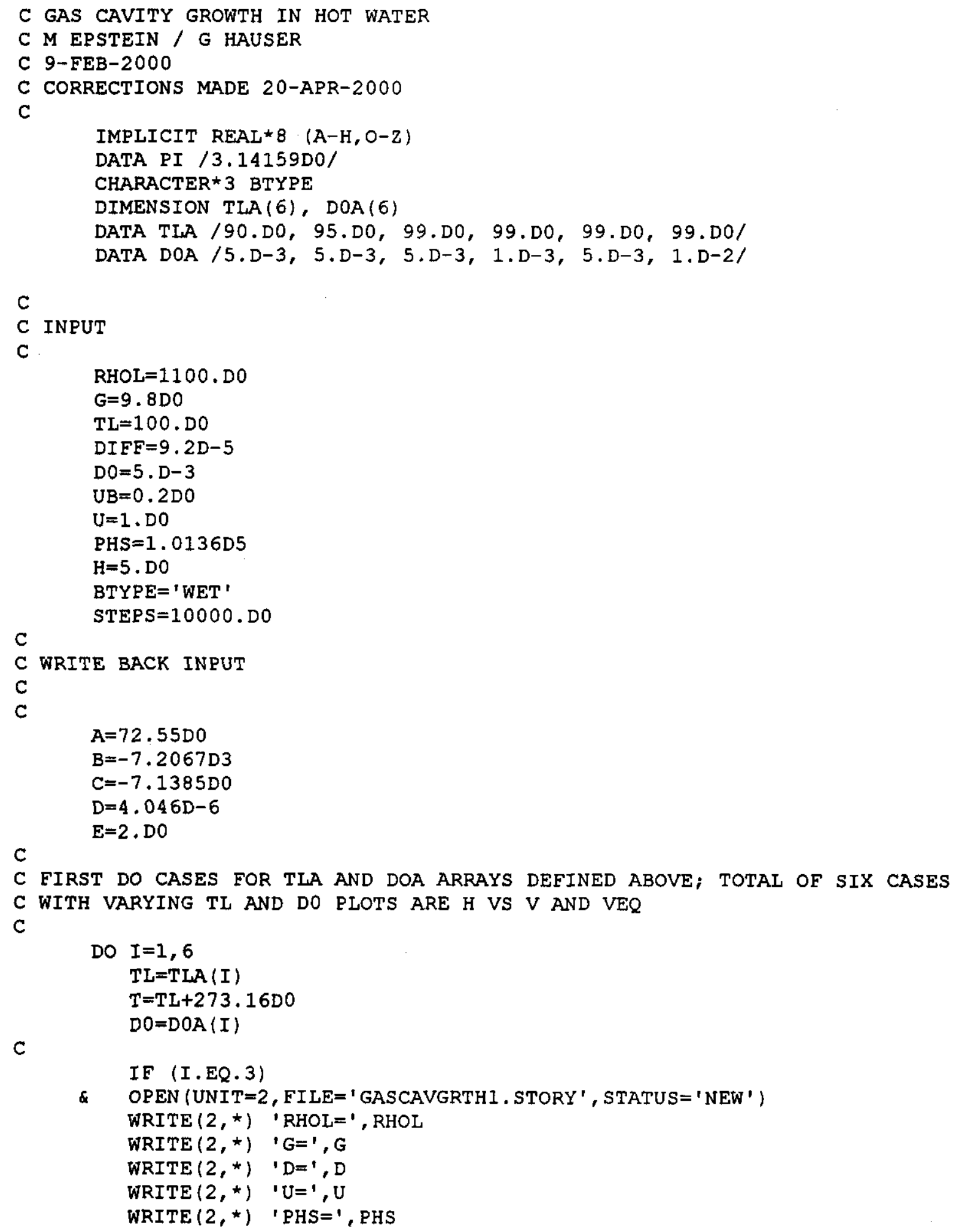




\section{RPP-6213 REV 0}

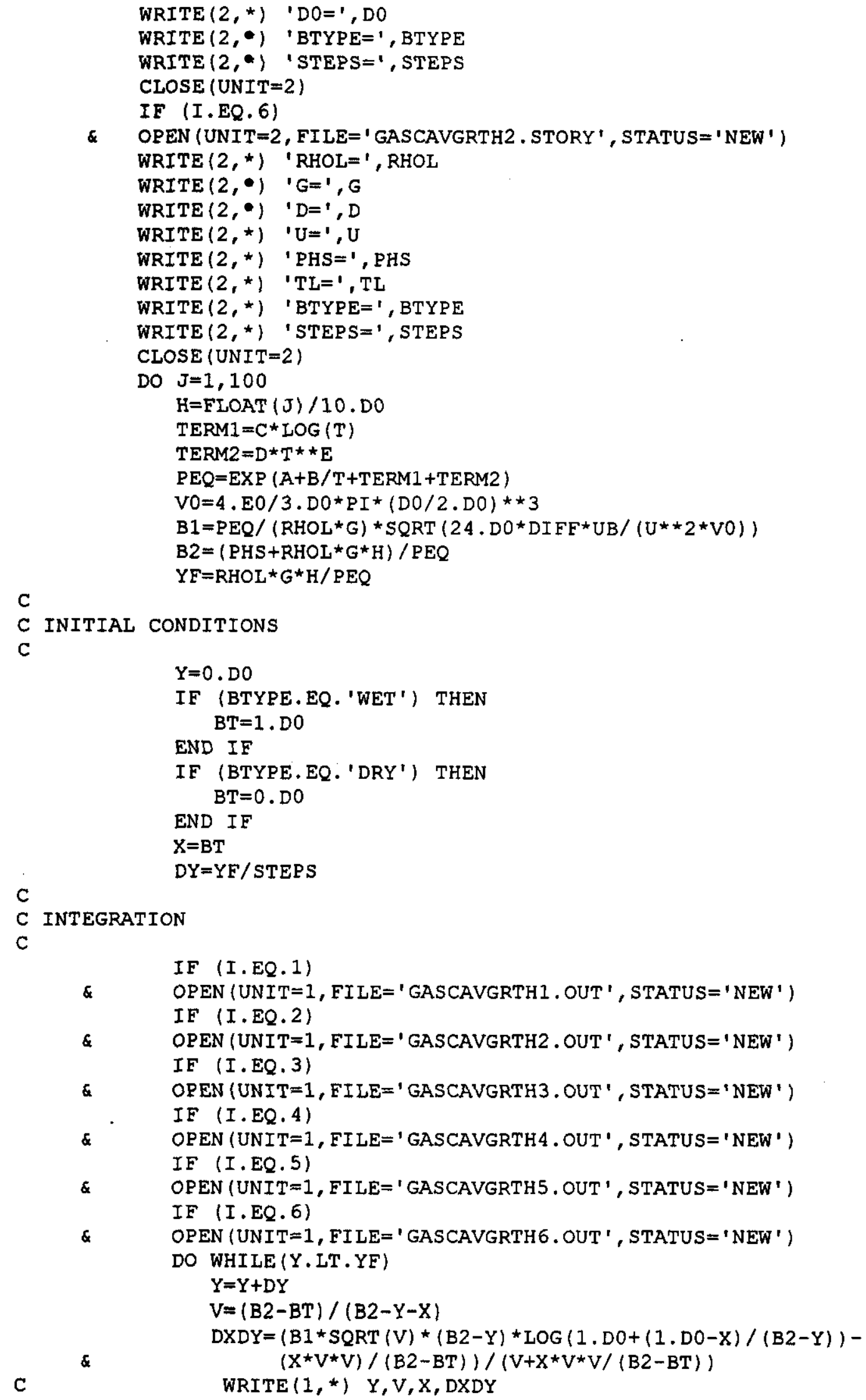




\section{RPP-6213 REV 0}

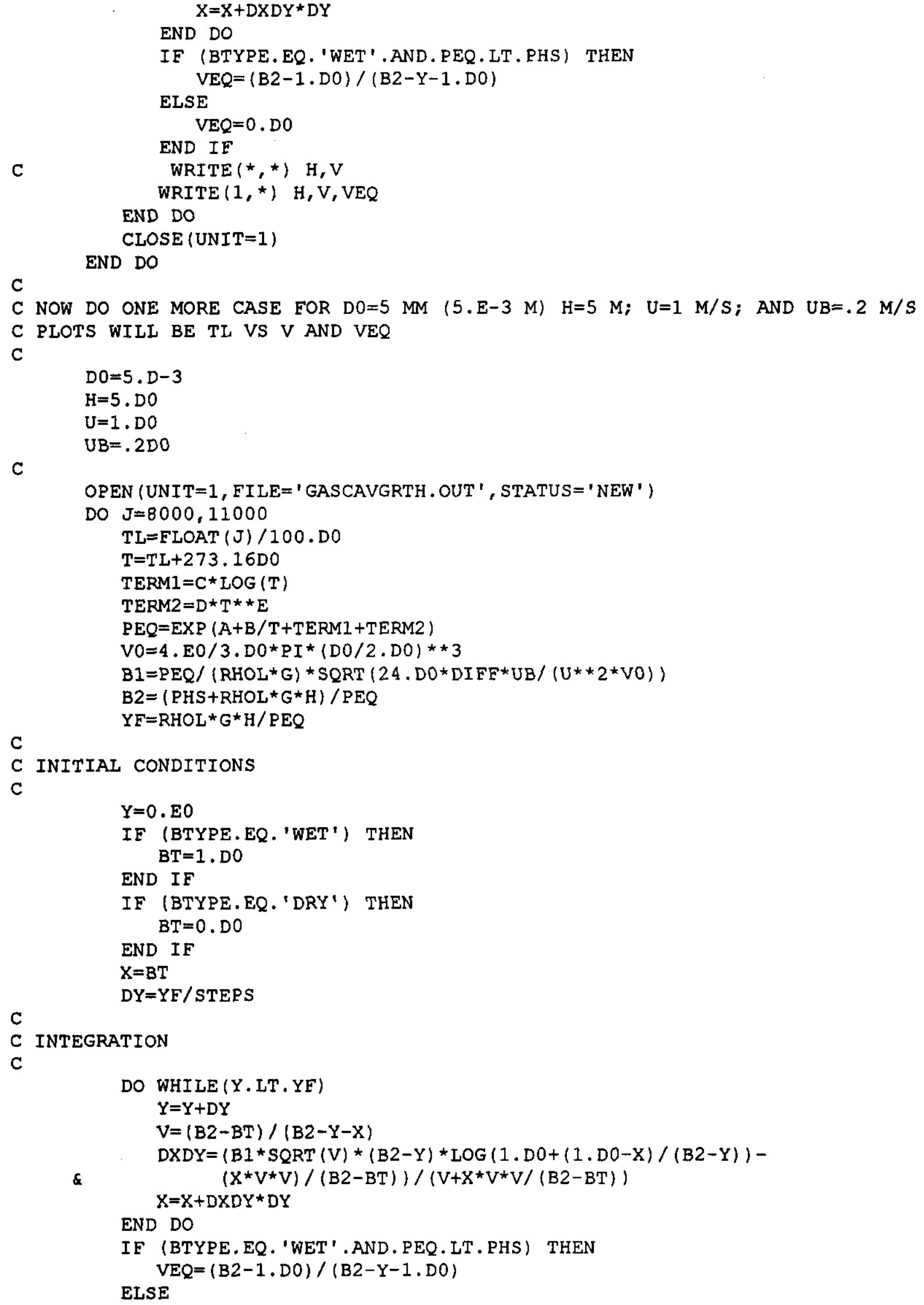




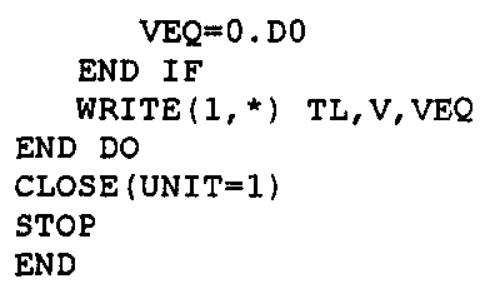

\section{D.2 GASCAVGRTH.GCL: Plot Script for Chapter 4 Figures}

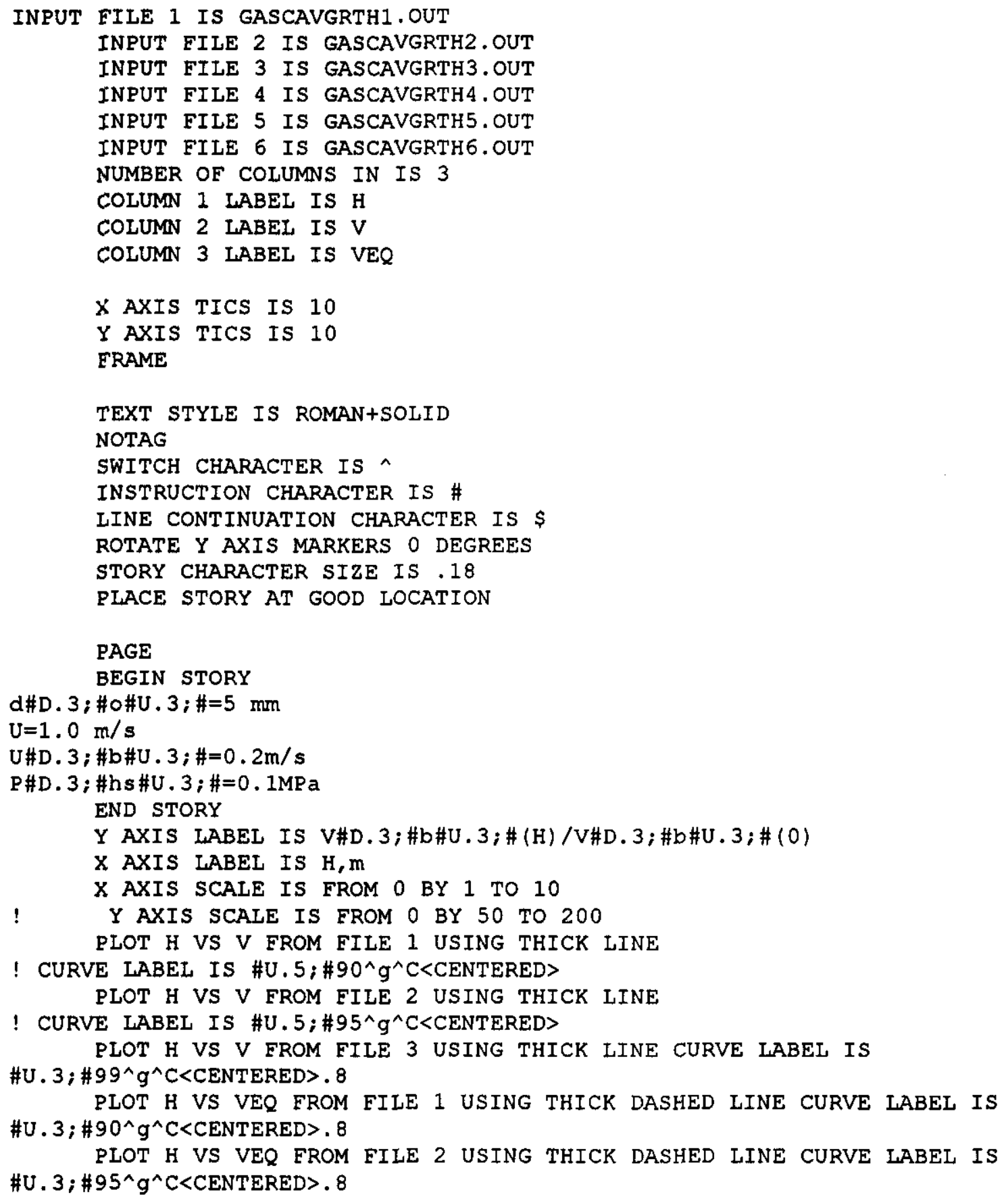




\section{RPP-6213 REV 0}

PLOT H VS VEQ FROM FILE 3 USING THICK DASHED LINE CURVE LABEL IS \#U. 3; \#T\#D. 3; \#^=^\#U. 3;\#=99^ $\mathrm{g}^{\wedge} \mathrm{C}<\mathrm{CENTERED}>.8$

! CAPTION IS Fig.4-3 Bubble expansion ratio versus depth of supernatent pool; pool temperature\$

! T\#D.3;\#^=^\#U.3; as a parameter. Dashed curves refer to zero mass transfer resistance.

DISPLAYY

PAGE

BEGIN STORY

T\#D. $3 ; \#^{\wedge}=\wedge \# U .3 ; \#=99^{\wedge} \mathrm{g}^{\wedge} \mathrm{C}$

$\mathrm{U}=1.0 \mathrm{~m} / \mathrm{s}$

U\#D. $3 ; \# \mathrm{~b} \# U .3 ; \#=0.2 \mathrm{~m} / \mathrm{s}$

P\#D. 3; \#hs\#U. 3 ; \#=0.1MPa

END STORY

PLOT H VS V FROM EILE 4 USING THICK LINE CURVE LABEL IS

\#U. 3 ; \#d\#D. 3 ; \#O\#U . 3 ; \#=1mm<CENTERED>. 8

PLOT H VS $V$ FROM FILE 5 USING THICK LINE CURVE LABEL IS

$\#$ \#. 3; \#5m<CENTERED>. 8

PLOT H VS V FROM FILE 6 USING THICK LINE CURVE LABEL IS

\#U. 3;\#10mm<CENTERED>. 8

PLOT H VS VEQ FROM FILE 4 USING THICK DASHED LINE

! CAPTION IS Fig.4-4 Bubble expansion ratio versus depth of supernatent pool; initial bubbles

! diameter as a parameter. Dashed curve refers to zero mass transfer

resistance.

DISPLAY

PAGE

BEGIN STORY

d\#D. $3 ; \# \circ \# U .3 ; \#=5 \mathrm{~mm}$

$\mathrm{H}=5.0 \mathrm{~m}$

$\mathrm{U}=1.0 \mathrm{~m} / \mathrm{s}$

U\#D. $3 ; \# b \# U .3 ; \#=0.2 \mathrm{~m} / \mathrm{s}$

P\#D. 3 ; \#hs\#U. $3 ; \#=0.1 \mathrm{MPa}$

END STORY

INPUT FILE 1 IS GASCAVGRTH.OUT

NUMBER OF COLUMNS IS 3

COLUMN 1 LABEL IS TL

COLUMN 2 LABEL IS $V$

COLUMN 3 LABEL IS VEQ

$X$ AXIS SCALE IS FROM 80 BY 5 TO 110

$X$ AXIS TICS IS 5

$X$ AXIS LABEL IS Temperature, ${ }^{\wedge} g \wedge \mathrm{C}$

$Y$ AXIS SCALE IS FROM O BY 100 TO 800

PLOT TL VS MIN $(800, V)$ FROM FILE 1 USING THICK LINE

! CAPTION IS Fig.4-5 Bubble expansion ratio versus convective layer temperature $\left(<100^{\wedge} \mathrm{g}^{\wedge} \mathrm{C}\right)$ or $\$$

! buoyant parcel temperature T\#D.3;\#nc\#U.3; during buoyant displacement.

Dashed curve refers to\$

! zero mass transfer resistance.

DISPIAY

NO STORY

NO CAPTION

$X$ AXIS LABEL IS $\square$

$Y$ AXIS LABEL IS

FAI/00-14, Rev. 0

D-5

May 2000 
RPP-6213 REV 0

$X$ WINDOW IS FROM O TO 99.99

PLOT TL VS MIN (800,VEQ) FROM FILE 1 USING THICK DASHED LINE

$X$ AXIS SCALE IS SAME

$Y$ AXIS SCALE IS SAME

DISPLAY 


\section{E.0 CLUSTER ENTRAINMENT MODEL AND INPUT}

\section{E.1 TANK_BUMP.FOR: Chapter 5 Calculations}

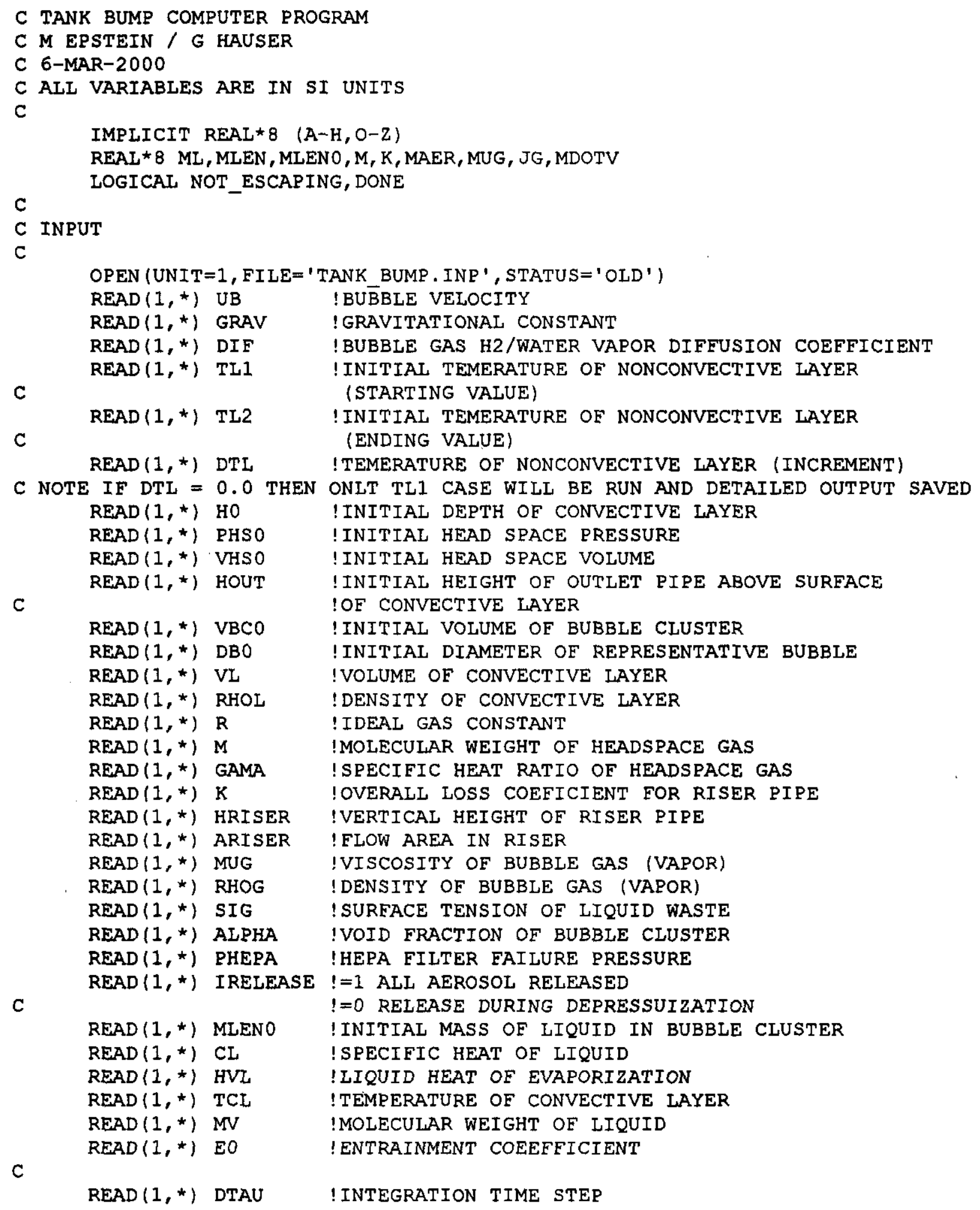




\section{RPP-6213 REV 0}

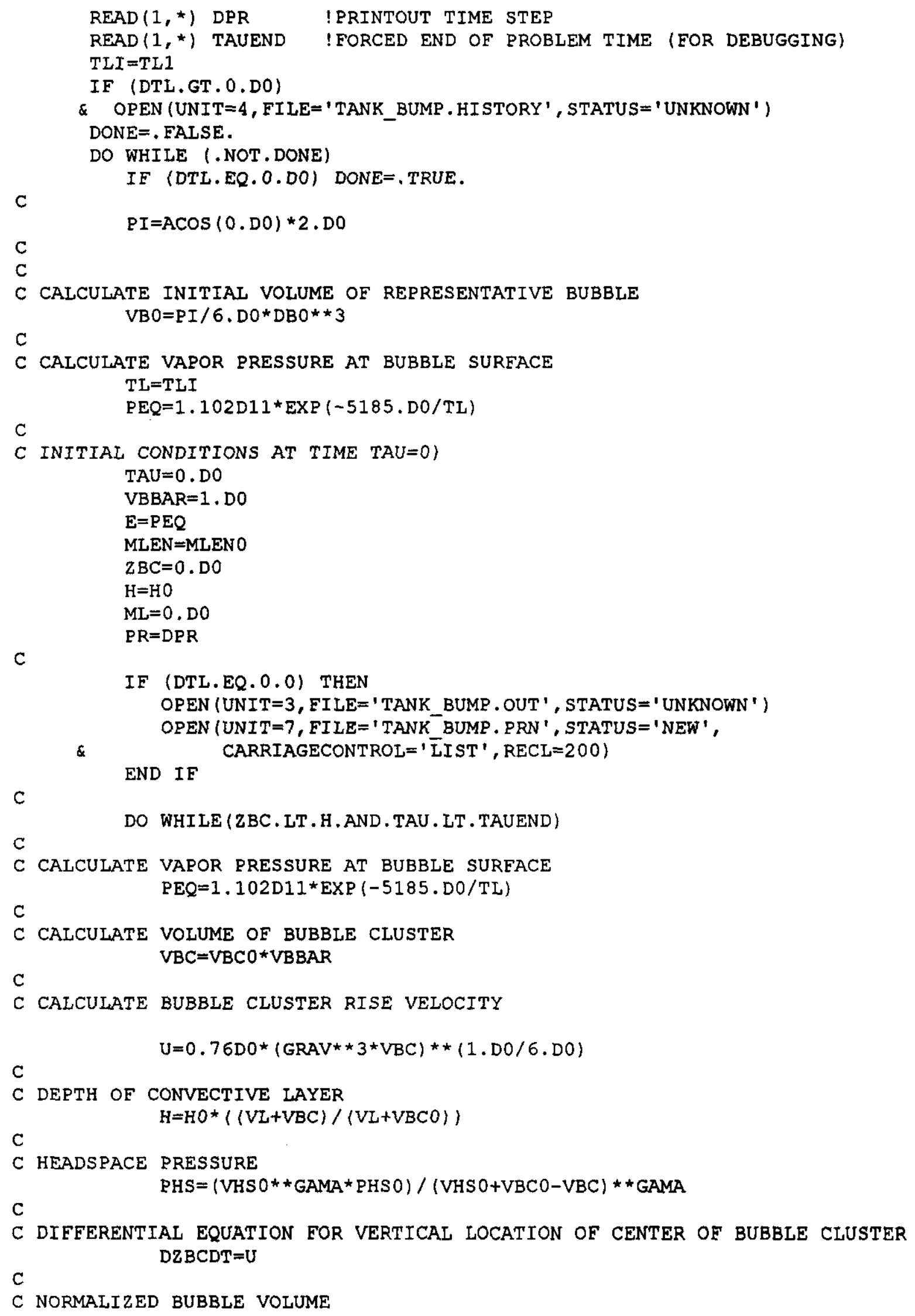




\section{RPP-6213 REV 0}

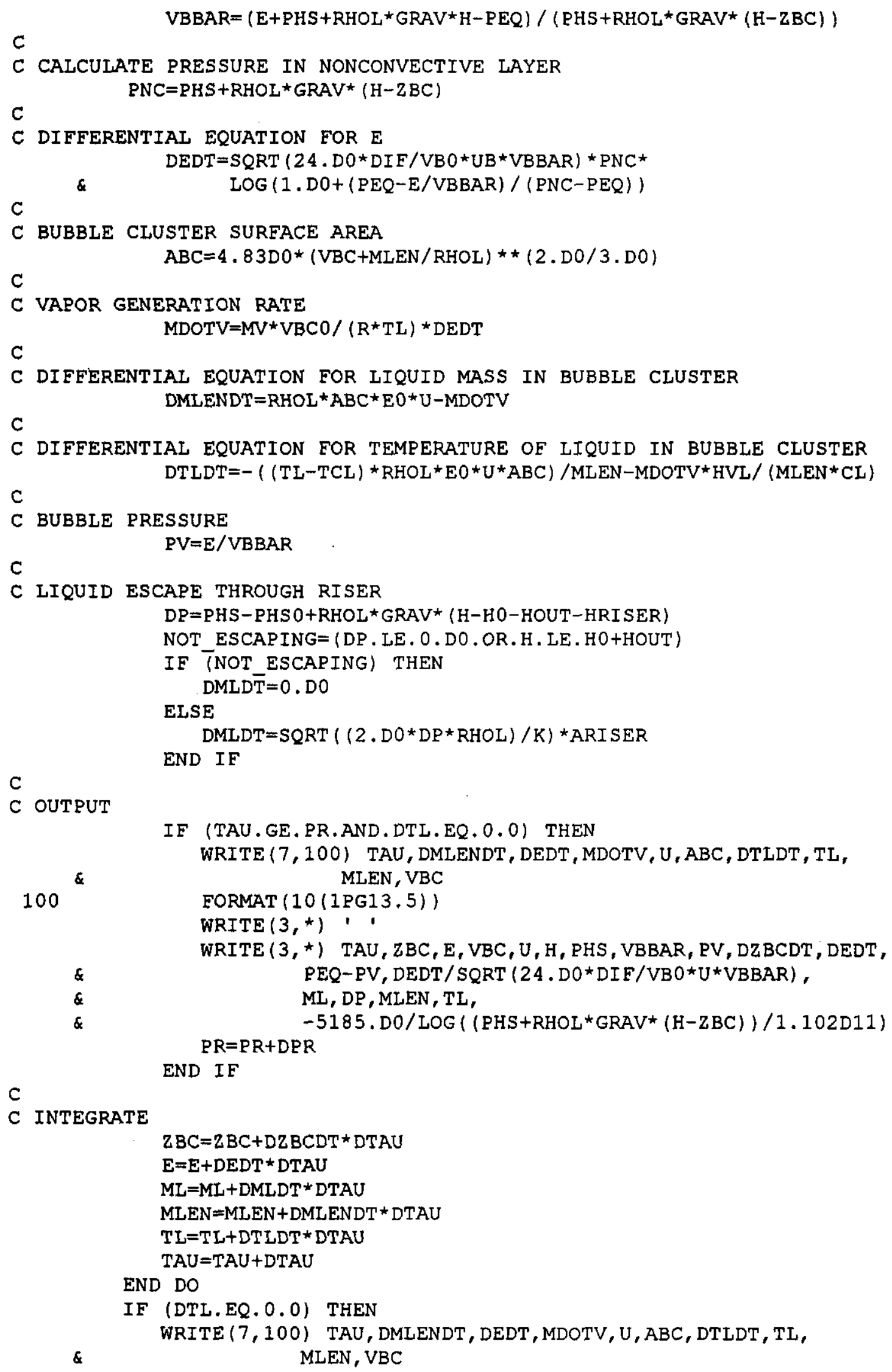




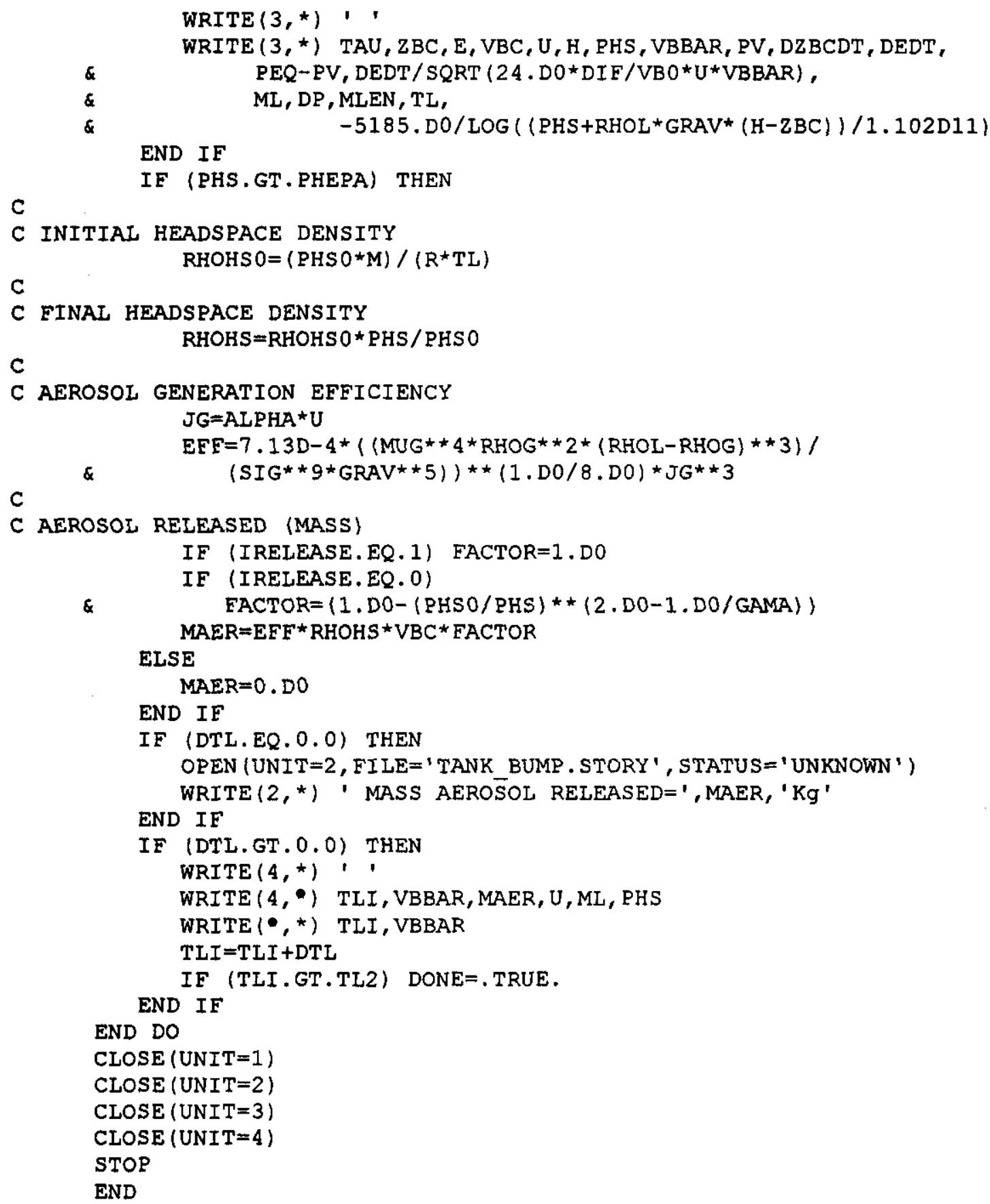

\section{E.2 TANK_BUMP.INP: Chapter 5 Input}

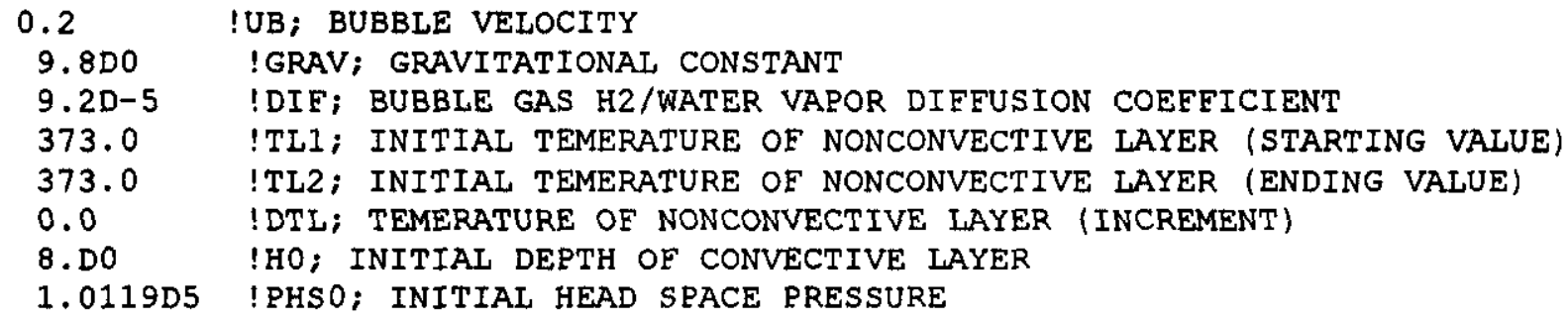




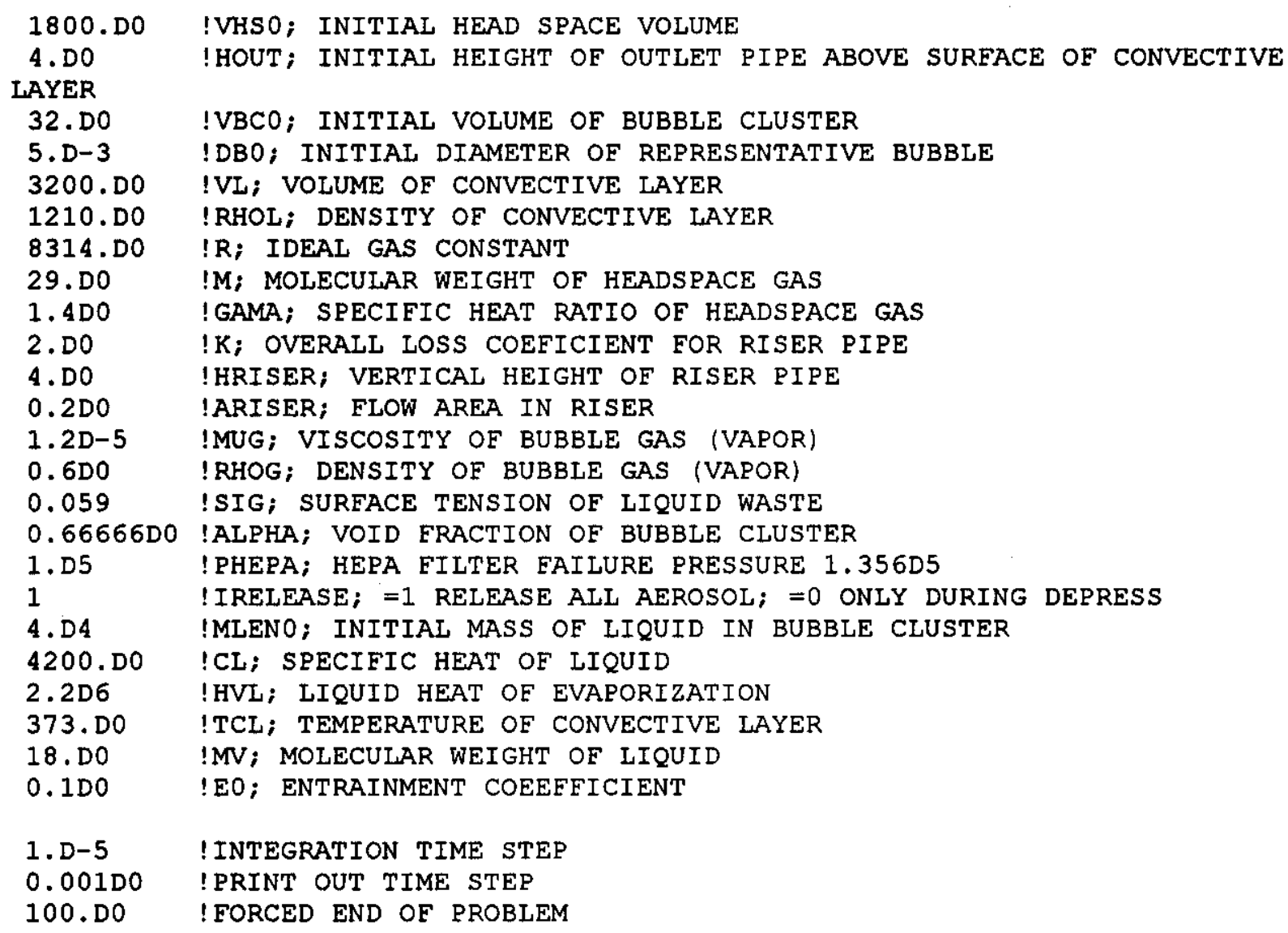

\title{
E.3 TANK_BUMP.GCL: Chapter 5 Plots
}

\author{
INPUT FILE 1 IS TANK BUMP.OUT \\ NUMBER OF COLUMNNS IN FILE 1 IS 18 \\ COLUMN 1 LABEL IN FILE 1 IS TAU \\ COLUMN 2 LABEL IN FILE 1 IS $\mathrm{ZBC}$ \\ COLUMN 3 LABEL IN FILE 1 IS E \\ COLUMN 4 LABEL IN FILE 1 IS VBC \\ COLUMN 5 LABEL IN FILE 1 IS U \\ COLUMN 6 LABEL IN EILE 1 IS $\mathrm{H}$ \\ COLUMN 7 LABEL IN FILE 1 IS PHS \\ COLUMN 8 LABEL IN FILE 1 IS VBBAR \\ COLUMN 9 LABEL IN FILE 1 IS PV \\ COLUMN 10 LABEL IN FILE 1 IS DZBCDT \\ COLUMN 11 LABEL IN FILE 1 IS DEDT \\ COLUMN 12 LABEL IN FILE 1 IS DIFE \\ COLUMN 13 LABEL IN FILE 1 IS DIEF2 \\ COLUMN 14 LABEL IN FILE 1 IS ML \\ COLUMN 15 LABEL IN FILE 1 IS DP \\ COLUMN 16 LABEL IN FILE 1 IS MLEN \\ COLUMN 17 LABEL IN FILE 1 IS TL \\ COLUMN 18 LABEL IN FILE 1 IS TEQ \\ TEXT STYLE IS ROMAN+SOLID \\ BEGIN STORY \\ INCLUDE FILE IS TANK_BUMP.INP
}


INCLUDE FIIE IS TANK_BUMP.STORY

END STORY

STORY CHARACTER SIZE IS .08

PLACE STORY AT GOOD LOCATION

! HARD COPY OFF

PAGE

$X$ AXIS IAABEL IS Time, sec

$Y$ AXIS LABEI IS ZBC, Vertical location of bubble cluster, $m$

$X$ AXIS TICS IS 10

$Y$ AXIS TICS IS 10

FRAME

PLOT TAU VS ZBC FROM FILE 1 USING LINE

DISPLAY

PAGE

NO STORY

Y AXIS LABEL IS Bubble pressure - volume PLOT TAU VS E FROM FILE 1 USING LINE DISPIAY

PAGE

$Y$ AXIS LABEL IS VBC, Volume of bubble cluster, $m^{\star *} 3$ PLOT TAU VS VBC FROM FILE 1 USING LINE

DISPLAY

PAGE

$Y$ AXIS LABEI IS $U$, Bubble cluster rise velocity, $\mathrm{m} / \mathrm{sec}$ PLOT TAU VS U FROM FILE 1 USING LINE

DISPLAY

PAGE

$Y$ AXIS LABEL IS $\mathrm{H}$, Depth of convective layer, $m$ PLOT TAU VS H FROM FILE 1 USING LINE

DISPLAY

PAGE

$Y$ AXIS LABEL IS PHS, Headspace pressure, Pa PLOT TAU VS PHS FROM FILE 1 USING LINE

DISPLAY

PAGE

Y AXIS LABEL IS VBBAR, Normalized bubble volume PLOT TAU VS VBBAR FROM FILE 1 USING LINE

DISPLAY

PAGE

$Y$ AXIS LABEL IS PV, Bubble pressure, Pa PLOT TAU VS PV FROM FILE 1 USING LINE

DISPLAY

PAGE

$Y$ AXIS LABEL IS DZBCDT, $\mathrm{m} / \mathrm{sec}$

PLOT TAU VS DZBCDT FROM FILE 1 USING LINE

DISPLAY 


\section{RPP-6213 REV 0}

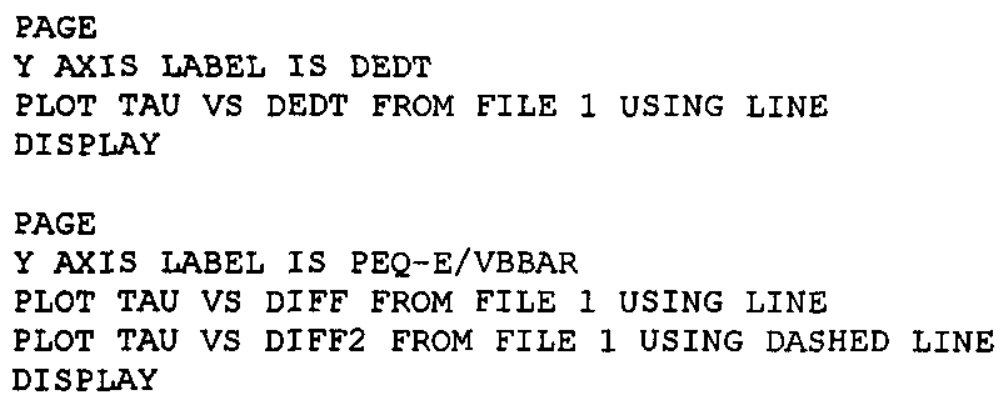

PAGE

Y AXIS LABEL IS ML, Mass of liquid escaping through riser, Kg PLOT TAU VS ML FROM FILE 1 USING LINE

DISPLAY

PAGE

Y AXIS LABEL IS MLEN, Mass of liquid in bubble, $\mathrm{Kg}$ PLOT TAU VS MLEN FROM EILE 1 USING LINE

DISPIAY

BEGIN STORY

d\#D. 3;\#O\#U. $3 ; \#=5 \mathrm{~mm}$

!P\#D. 3; \#hs\#U. $3 ; \#=0.1 \mathrm{MPa}$

!

U\#D. $3 ; \# b \# U .3 ; \#=0.2 \mathrm{~m} / \mathrm{s}$

M\#D. 3; \#^^^^\#U. $3 ; \#(0)=4000 \mathrm{Kg}$

V\#D. 3;\#b\#U. $3 ; \#(0)=32 \mathrm{m \# U} \cdot 3 ; \# 3$

END STORY

STORY CHARACTER SIZE IS . 2

PLACE STORY AT GOOD LOCATION

TEXT STYLE IS ROMAN+SOLID

ROTATE $Y$ AXIS MARKERS 0 DEGREES

SWITCH CHARACTER IS ^

INSTRUCTION CHARACTER IS \#

Y AXIS SCALE IS FROM 370 BY 5 TO 395

$X$ AXIS TICS IS 5

$Y$ AXIS TICS IS 5

NOTAG

! HARD COPY ON

PAGE

Y AXIS LABEL IS Temperature, $\mathrm{K}$

PLOT TAU VS TL FROM FILE 1 USING THICK LINE CURVE LABEL IS

\#U. $5 ; \# T \# D .3 ; \# \wedge=\wedge<C E N T E R E D>.3$

PLOT TAU VS TEQ FROM FILE 1 USING THICKDASHED LINE CURVE LABEL IS \#U . 5;\#T\#D. 3 ; \#bP<CENTERED>. 3

! CAPTION IS Fig.4-6 Temperature history of buoyant parcel compared with its boiling temperature. DISPLAY

! PAGE 
RPP-6213 REV 0

$Y$ AXIS LABEL IS DP

PLOT TAU VS DP FROM FILE 1 USING LINE

DISPIAY 


\section{F.0 HADCRT CHANGES AND INPUT}

\section{F.1 BUMP.FOR: Addition to HADCRT for Chapter 8}

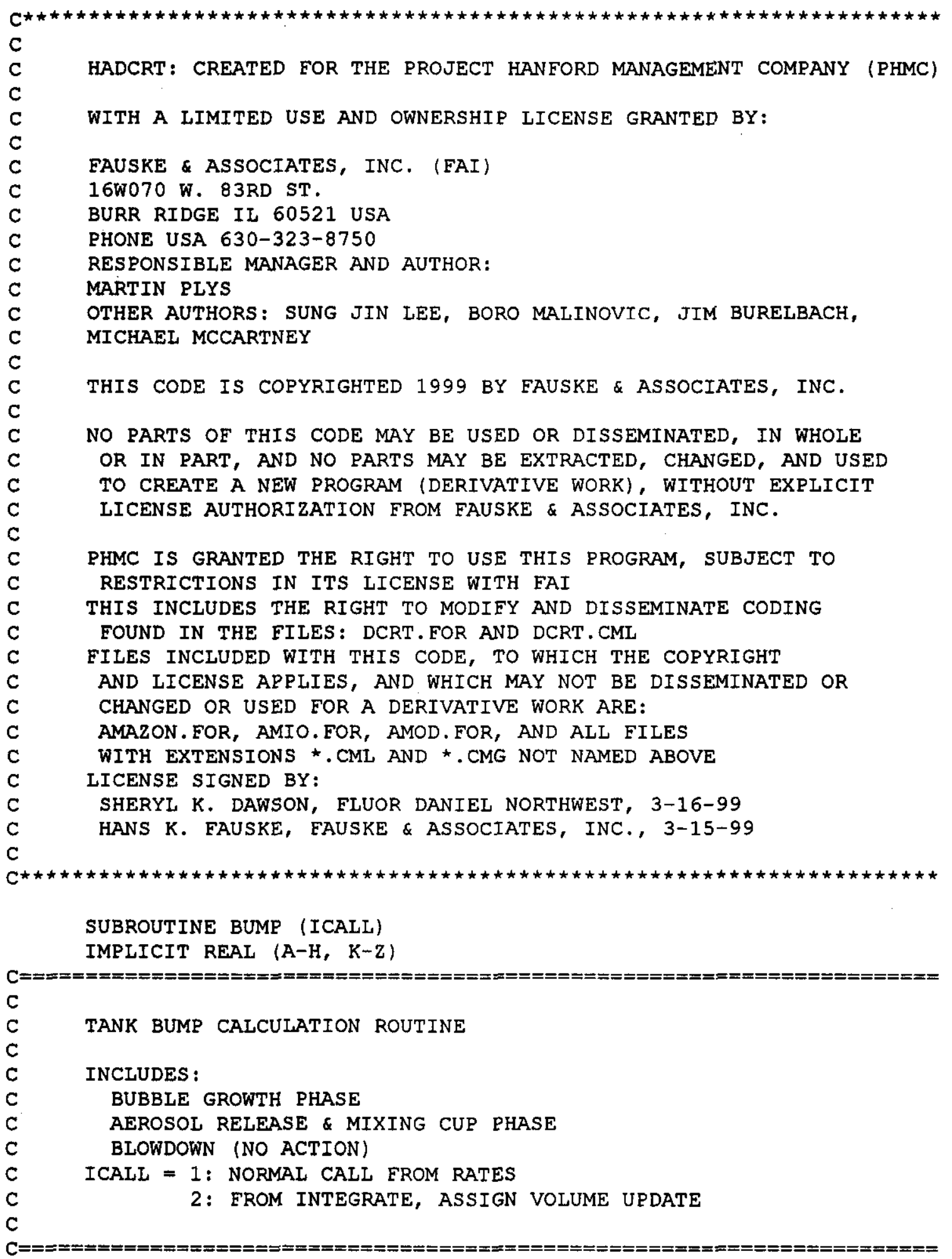




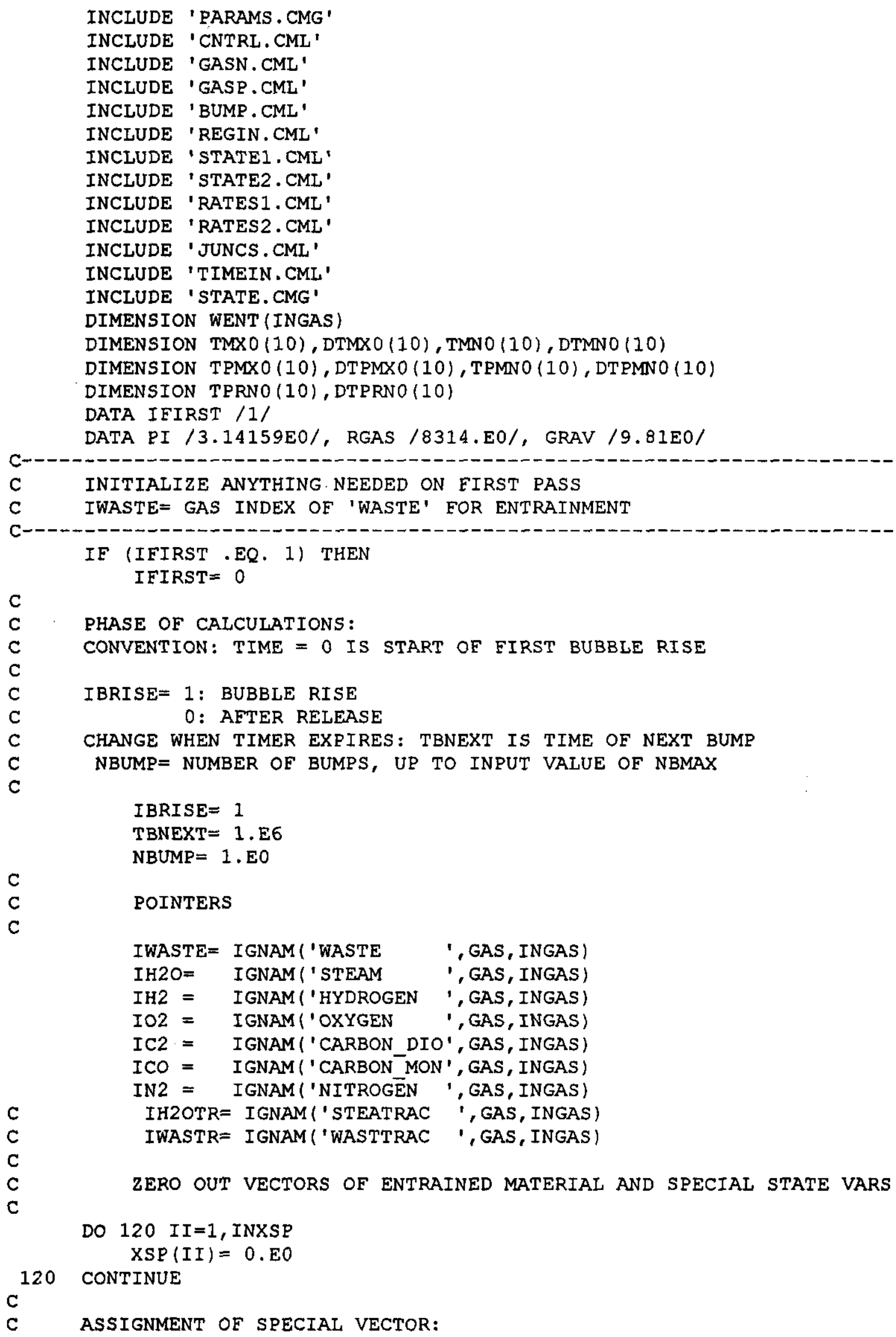




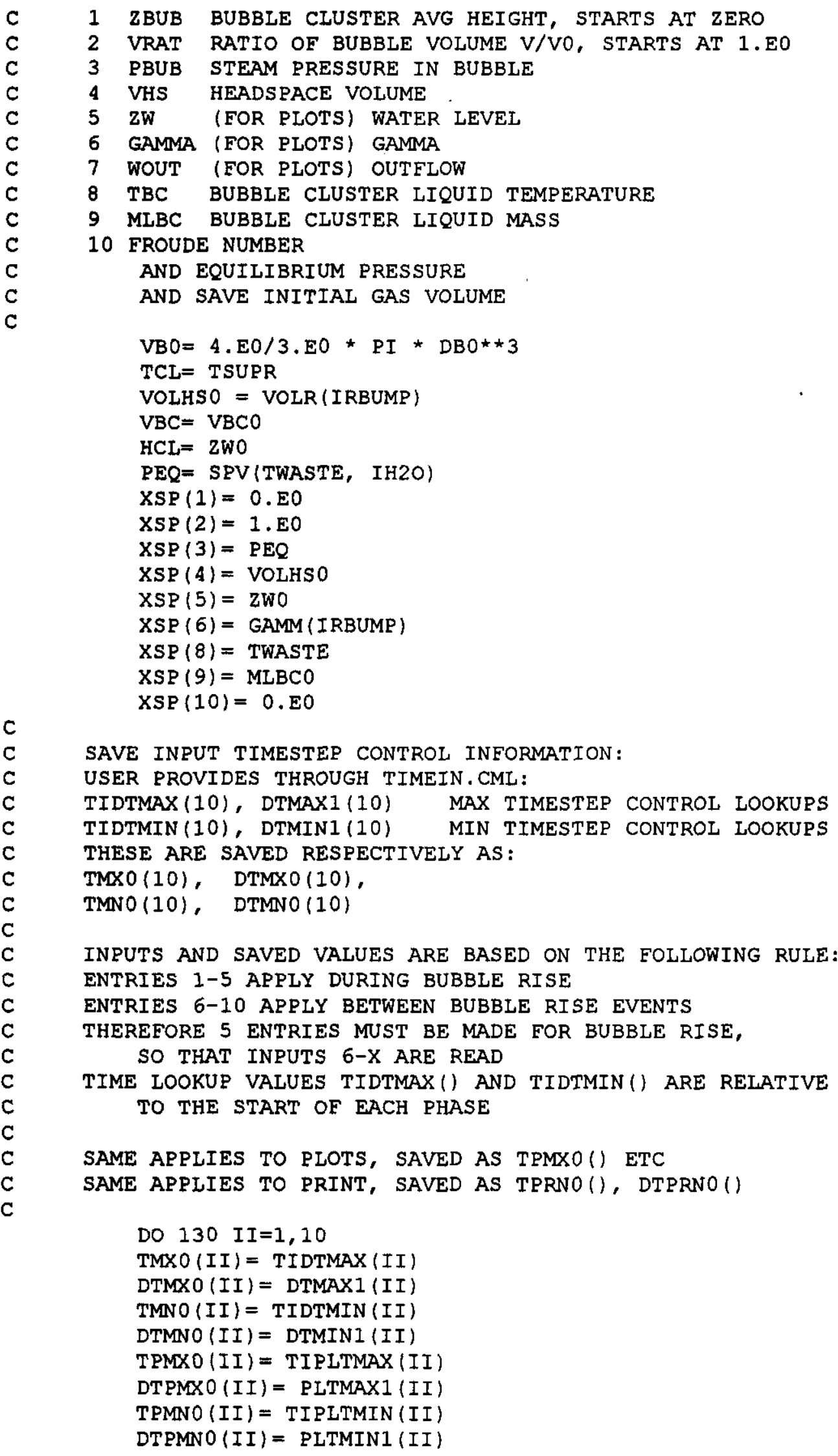




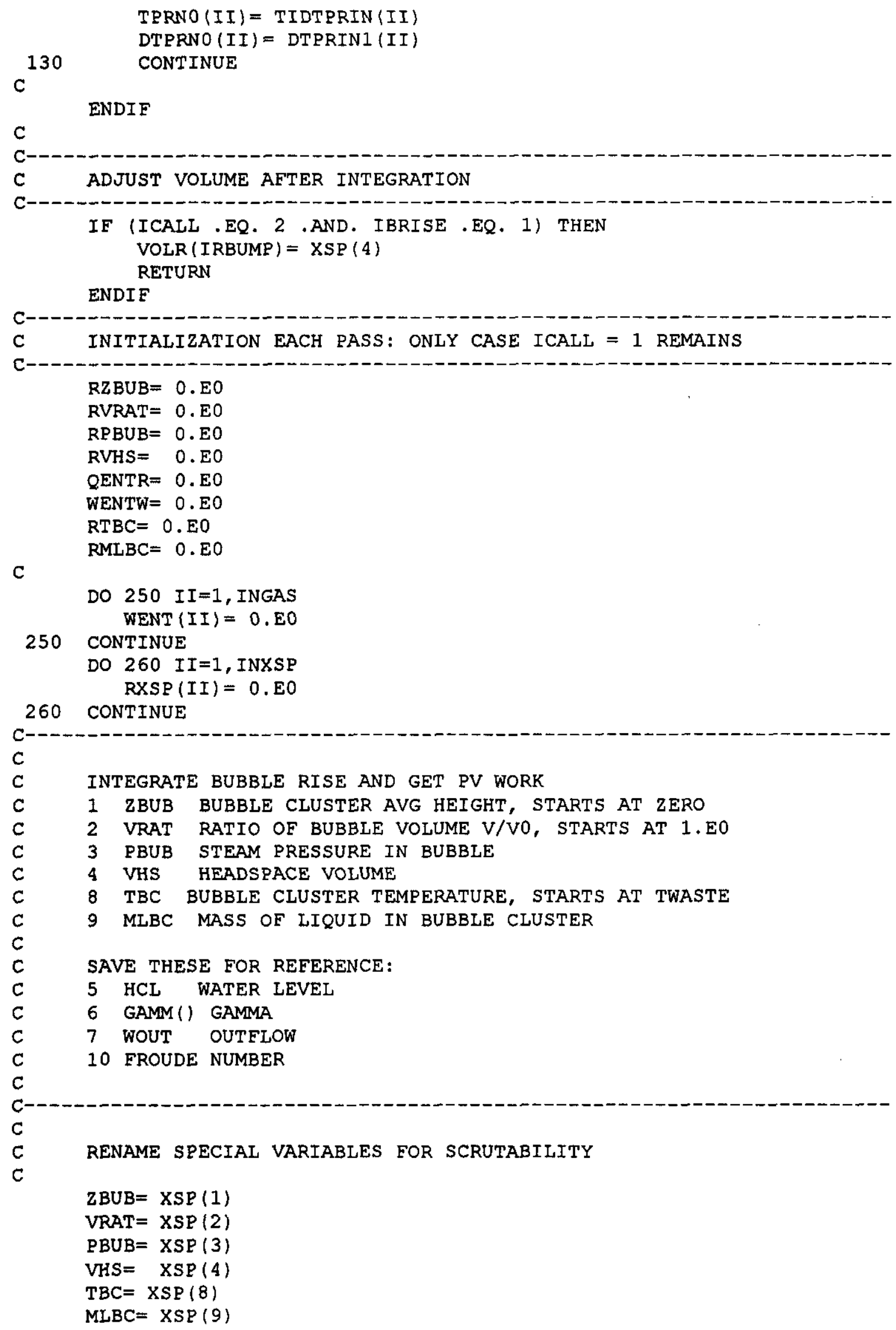




\section{RPP-6213 REV 0}

C

C

C

C

C

C

C

C

C

C

$\mathrm{C}$

c

$\mathrm{C}$

c

C

$\mathrm{C}$

$\mathrm{C}$

DECIDE ON PHASE OF PROBLEM:

NEED AUXILIARY RELATIONS IF DURING RISE PHASE

IF (IBRISE .EQ. 1) THEN

$\mathrm{VBC}=\mathrm{VBCO}$ - VRAT

ENDIF

$\mathrm{HCL}=2 \mathrm{WO} *(\mathrm{VLIQ}+\mathrm{VBC}) /(\mathrm{VLIQ}+\mathrm{VBCO})$

TRANSITION BETWEEN PHASES: END OF BUMP

ADD AEROSOL, MIX BUBBLE AND HEADSPACE,

NEW PRESSURE \& TEMPERATURE

IF (IBRISE .EQ. 1 .AND. ZBUB .GE. HCL) THEN

$I R=I R B U M P$

IBRISE $=0$

TBNEXT $=$ TIME + TINTB

$\operatorname{XSP}(5)=\mathrm{HCL}$

$X S P(6)=G A M M(I R)$

$\operatorname{XSP}(10)=0 . E O$

C ENTRAINED WASTE

UBUB $=0.76 * \mathrm{SQRT}(\mathrm{GRAV}) \cdot \mathrm{VBC} \star *(1 . \mathrm{E} 0 / 6 . \mathrm{E} 0)$

USUP $=0.6666 \mathrm{E} O *$ UBUB

EGROUP $=($ MUGR $(I R) \star \star 4 *$ RHOG $(I R) \star \star 2 *($ RHOLIQ-RHOG $(I R)) \star \star 3)$ / (SURWAS $\star \star 9 \star$ GRAV $\star \star 5$ )

ENT $=$ FBENT $\star 7.13 E-4 *$ EGROUP $\star \star 0.125 \bullet$ USUP $\star \star 3$

MAER $=E N T * R H O G(I R) * V B C$

STEAM AND WASTE ENTHALPIES; USE WASTE AT HEADSPACE TEMP

WENT (IWASTE $)=1 . \mathrm{E} 0$

HWASTE $=$ HGSRC $(2$, PR (IRBUMP $), T B C$, WENT, $0 . E 0)$

WENT ( IWASTE $)=0$. EO

WENT $(I H 2 O)=1$. EO

$\operatorname{HSTM}=\operatorname{HGSRC}(1, P R(I R), T B C$, WENT, $0, E 0)$

WENT $(I H 2 O)=0 . E 0$

C MIXING CUP VALUES

RHOSTM $=P R(I R) * M W G(I H 2 O) /$ RGAS $/$ TWASTE

$M G(I H 2 O, I R)=M G(I H 2 O, I R)+V B C \star R H O S T M$

MA (IWASTE, IR $)=$ MA (IWASTE, IR + MAER

$U G(I R)=U G(I R)+M A E{ }^{\star}$ HWASTE + HSTM*VBC*RHOSTM

$\operatorname{VOLR}(I R)=$ VOLHSO + VBCO

$X S P(4)=$ VOLHSO + VBCO

$T$ TEES $=T$ TGR (IRBUMP)

PGES = PR (IRBUMP)

CALL GAST (1, IREG, IGAS, MWG, MG, MA, MFG, NFG, MGT, NGT, MAT,

อ FWG)

CALL PTREG1

I (VOLR (IR), TGES, PGES, MG, UG (IR), IR, TIME,

I MFG, NFG, MGT (IR), NGT (IR), MA, ML, UL (IR),

$0 \quad T G R(I R), P R$ (IR) , PPG, RHOG (IR) , DPDT (IR), MCE (IR) , DPDMG,

$0 \quad U G I, H G I, T L R(I R), U A G(I R), D P D V(I R)$,

O CPGR (IR), GAMM(IR), HLSAT, RHOL, IPTREG)

TIMESTEP CONTROL FOR PERIOD BETWEEN BUMPS, SAVED IN PLACES $6-10$

DO 332 II=I, 5

TIDTMAX (II) $=$ TIME + TMXO (II+5)

FAI/00-14, Rev. 0 


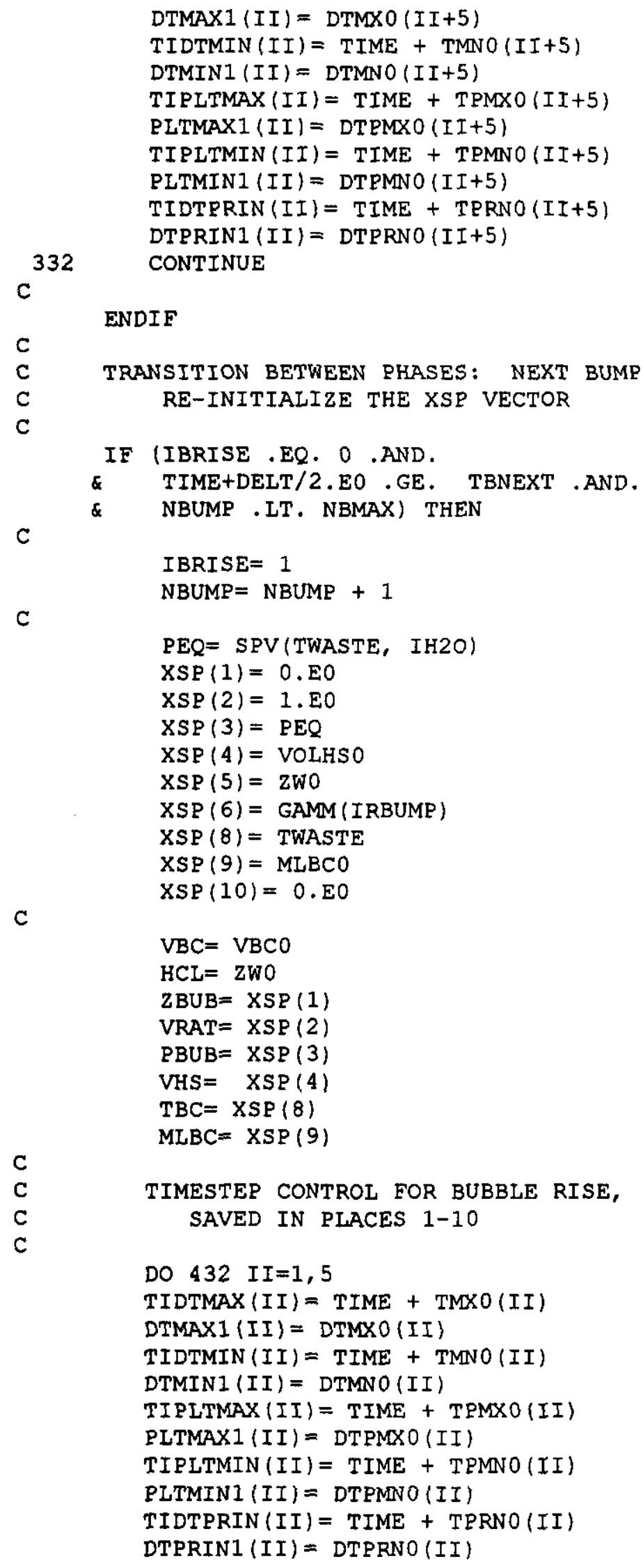

C CONTINUE

ENDIF

C

C TRANSITION BETWEEN PHASES: NEXT BUMP

C RE-INITIALIZE THE XSP VECTOR

C

IF (IBRISE .EQ. 0 .AND.

\& TIME+DELT/2.EO .GE. TBNEXT .AND.

C NBUMP . LT . NBMAX) THEN

IBRISE $=1$

NBUMP $=$ NBUMP +1

C

$P E Q=S P V(T W A S T E$, IH2O)

$\operatorname{XSP}(1)=0 . E 0$

$\operatorname{XSP}(2)=1 . E 0$

$X S P(3)=P E Q$

$X S P(4)=$ VOLHSO

$\operatorname{XSP}(5)=2 W 0$

$\mathrm{XSP}(6)=\mathrm{GAMM}($ IRBUMP $)$

$\mathrm{XSP}(8)=$ TWASTE

$\mathrm{XSP}(9)=\mathrm{MLBCO}$

$\operatorname{XSP}(10)=0 . E 0$

C

$\mathrm{VBC}=\mathrm{VBCO}$

$\mathrm{HCL}=\mathrm{ZWO}$

ZBUB $=X S P(1)$

VRAT $=X S P(2)$

PBUB $=X S P(3)$

$\mathrm{VHS}=\mathrm{XSP}(4)$

$\mathrm{TBC}=\mathrm{XSP}(8)$

$M L B C=X S P(9)$

C

TIMESTEP CONTROL FOR BUBBLE RISE, SAVED IN PLACES 1-10

DO $432 I I=1,5$

$T I D T M A X(I I)=T I M E+T M X O(I I)$

DTMAX1 $(I I)=\operatorname{DTMXO}(I I)$

$\operatorname{TIDTMIN}(I I)=\operatorname{TIME}+\operatorname{TMNO}(I I)$

DTMIN1 $(I I)=$ DTMNO(II)

TIPLTMAX $(I I)=$ TIME + TPMXO(II)

PLTMAXI (II) $=$ DTPMXO (II)

TIPLTMIN $(I I)=T I M E+T E M N O(I I)$

PLTMINI $(I I)=$ DTPMNO (II)

TIDTPRIN $(I I)=T I M E+T P R N O(I I)$

DTPRIN1 (II) $=$ DTPRNO (II) 


\section{RPP-6213 REV 0}

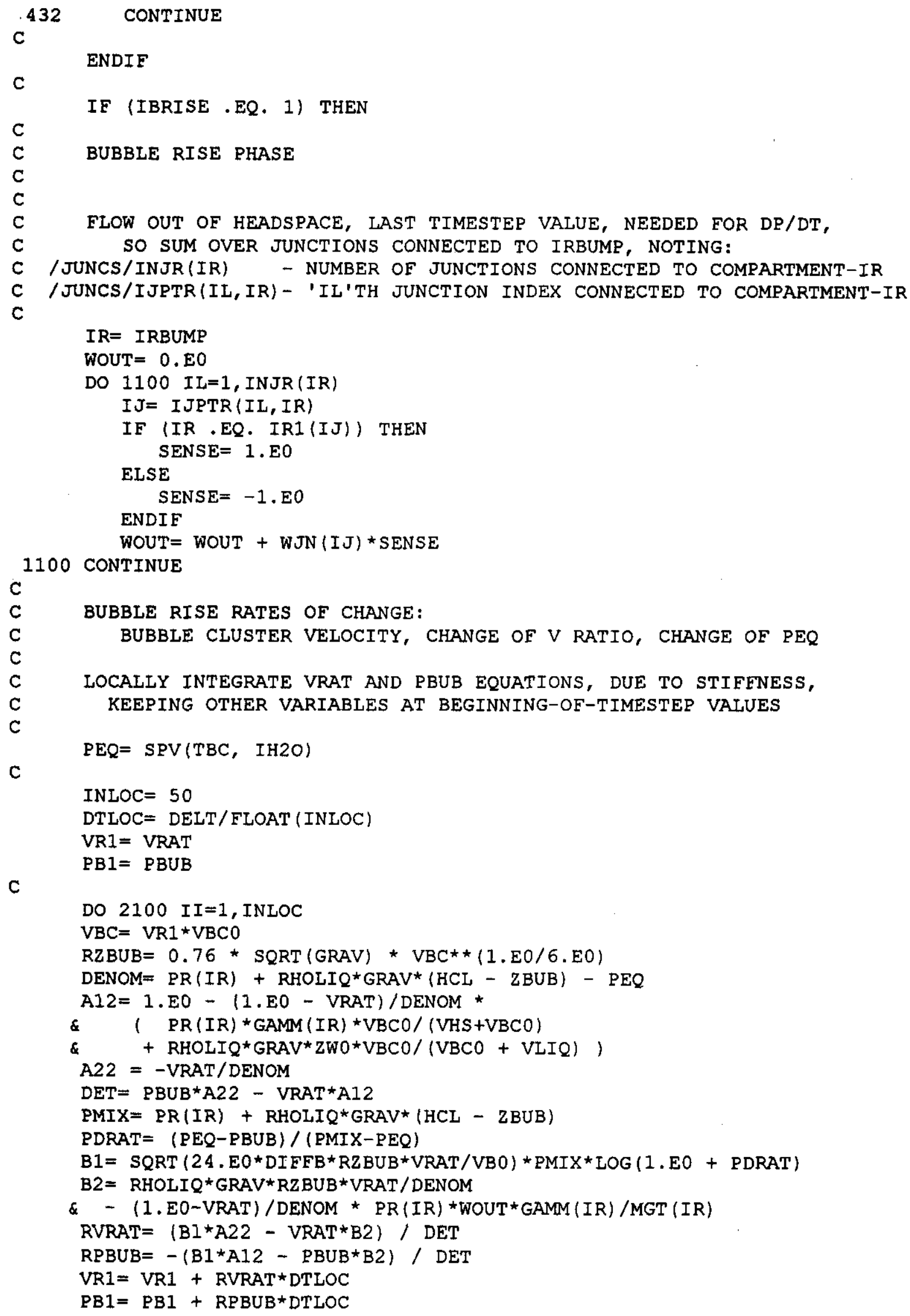




\section{RPP-6213 REV 0}

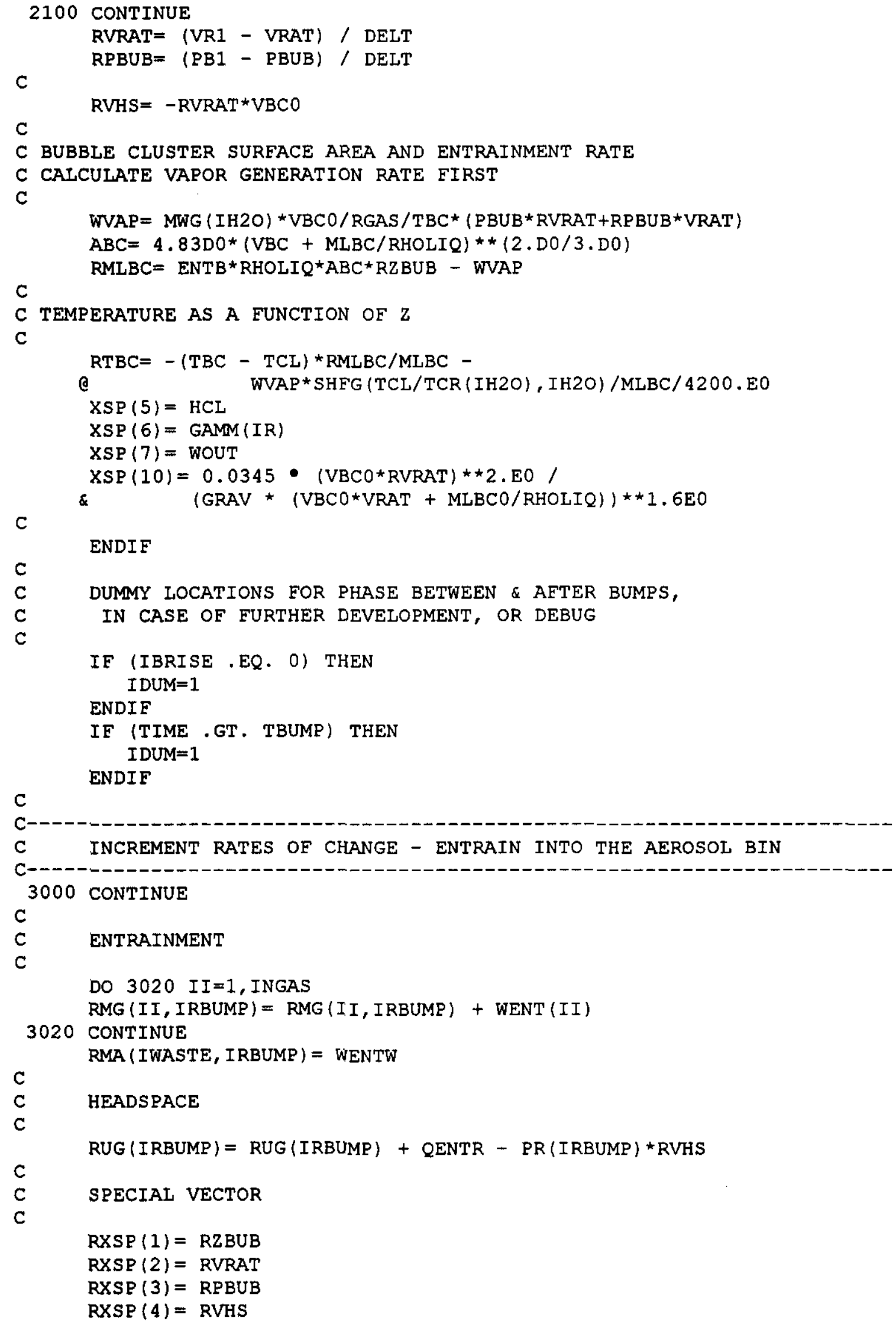




\section{RPP-6213 REV 0}

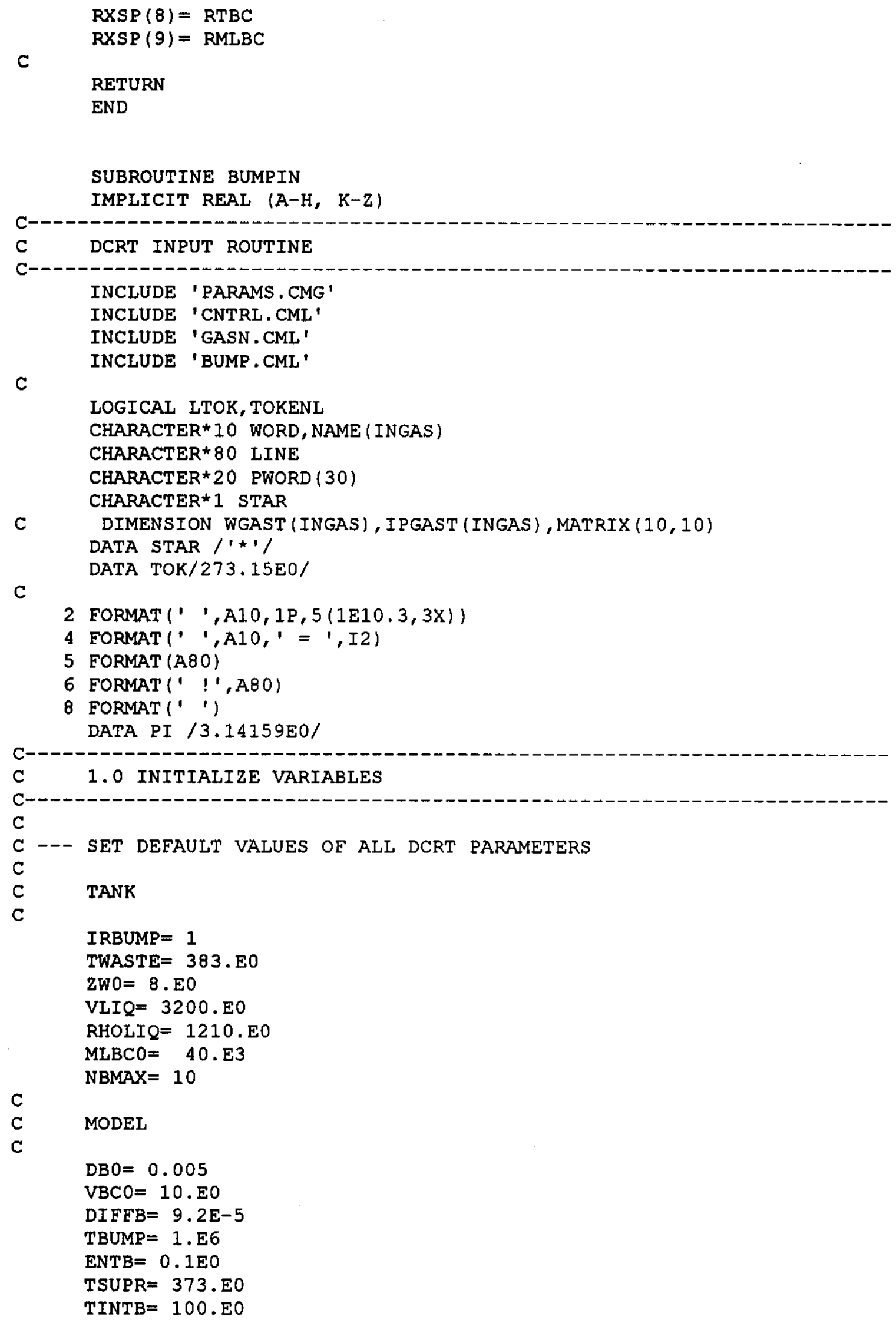




\section{RPP-6213 REV 0}

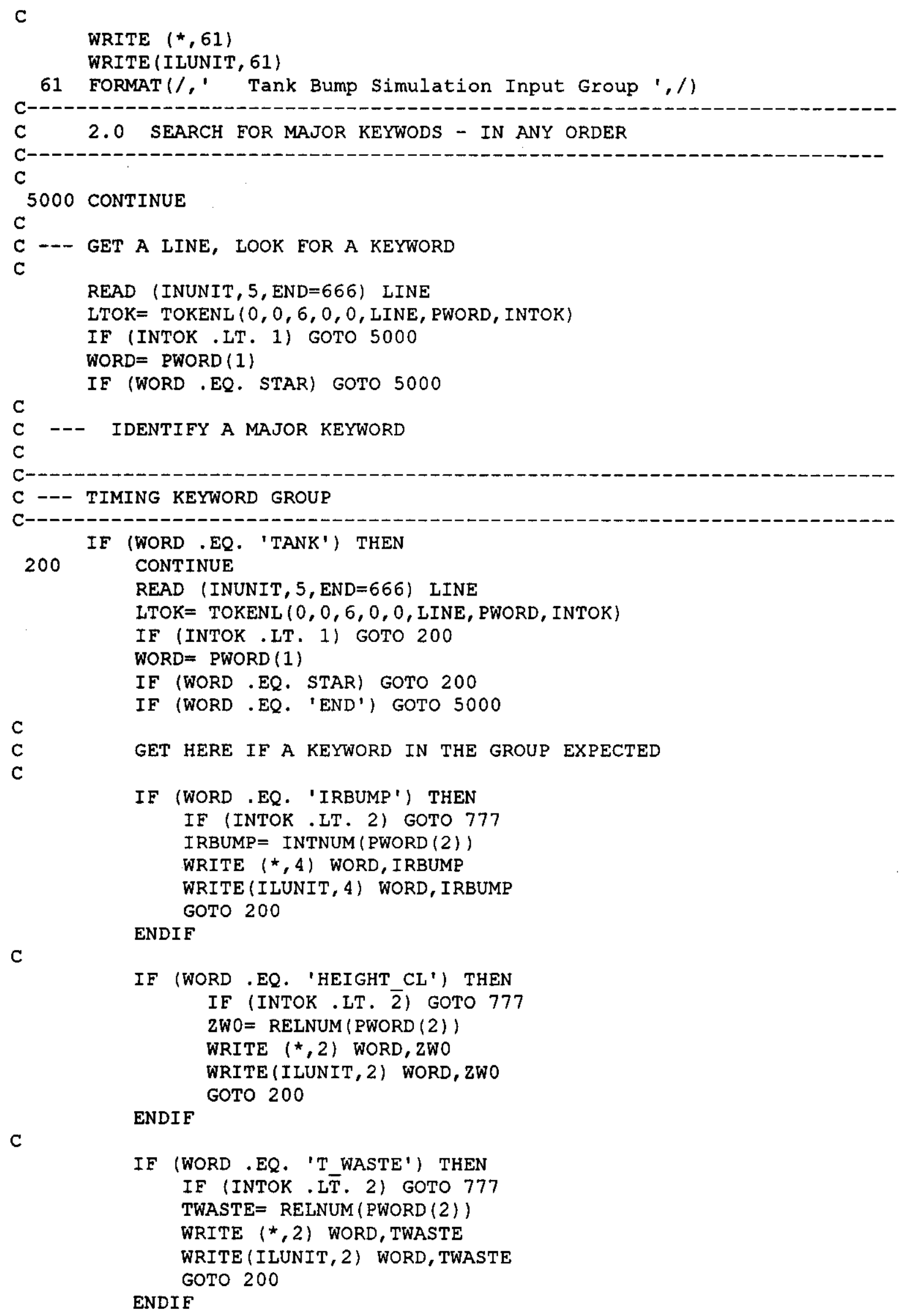




\section{RPP-6213 REV 0}

C

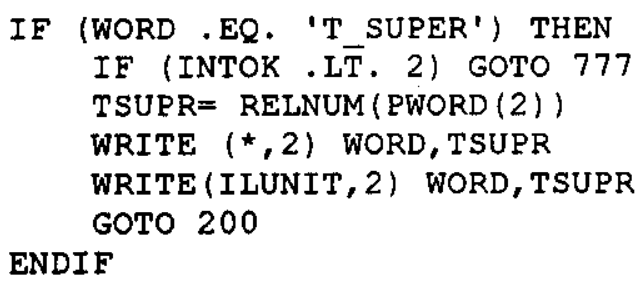

C

IE (WORD .EQ. 'VOLUME CL') THEN

IF (INTOK .LT. $\overline{2}$ ) GOTO 777

VLIQ $=$ RELNUM $($ PWORD (2) )

WRITE $(*, 2)$ WORD, VLIQ

WRITE (ILUNIT, 2) WORD, VLIQ

GOTO 200

ENDIF

C

IF (WORD .EQ. 'GAS VOL') THEN

IF (INTOK.LT. 2) GOTO 777

VBCO $=$ RELNUM (PWORD (2))

WRITE $(*, 2)$ WORD, VBCO

WRITE (ILUNIT, 2) WORD, VBC0

ENDIF

GOTO 200

C

IF (WORD .EQ. 'MASS LIQ') THEN

IF (INTOK .LT. $\overline{2}$ ) GOTO 777

MLBCO $=$ RELNUM $(\operatorname{PWORD}(2))$

WRITE $(*, 2)$ WORD, MLBCO

WRITE (ILUNIT, 2) WORD, MLBCO

GOTO 200

C

ENDIF

IF (WORD . EQ. 'NBMAX') THEN

IF (INTOK . LT. 2) GOTO 777

NBMAX $=$ RELNUM $($ PWORD $(2))$

WRITE $(*, 2)$ WORD, NBMAX

WRITE (ILUNIT, 2) WORD, NBMAX

GOTO 200

ENDIF

C

C

C

C --- FALL THROUGH HERE IF A COMMENT PRESENT NOT STARTING WITH STAR

WRITE $(*, 6)$ LINE

WRITE (ILUNIT, 6) LINE

GOTO 200

ENDIF

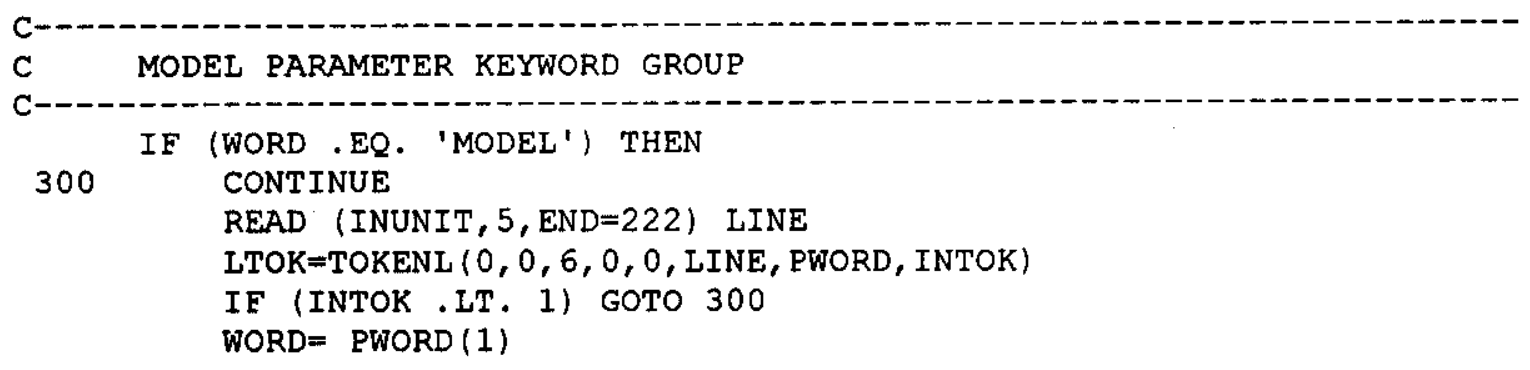




\section{RPP-6213 REV 0}

C

IF (WORD .EQ. STAR) GOTO 300

IF (WORD .EQ. 'END') GOTO 5000

IF (WORD . EQ. 'BUB DIAM') THEN

IF (INTOK .LT. 2) GOTO 777

$\mathrm{DB} 0=$ RELNUM (PWORD (2))

WRITE $(*, 2)$ WORD, DBO

WRITE (ILUNIT, 2) WORD, DBO

GOTO 300

c

ENDIF

IF (WORD .EQ. 'DIFFB') THEN

IF (INTOK .IT. 2) GOTO 777

DIFFB $=$ RELNUM (PWORD (2))

WRITE $(*, 2)$ WORD, DIFFB

WRITE (ILUNIT, 2 ) WORD, DIFFB

GOTO 300

C

ENDIF

IF (WORD . EQ. 'FBENT') THEN

IF (INTOK .LT. 2) GOTO 777

FBENT $=$ RELNUM $($ PWORD $(2))$

WRITE $(*, 2)$ WORD, FBENT

WRITE (ILUNIT, 2 ) WORD, FBENT

ENDI F

GOTO 300

C

IF (WORD . EQ. 'ENTB') THEN

IF (INTOK .LT. 2) GOTO 777

ENTB = RELNUM(PWORD (2))

WRITE $(*, 2)$ WORD, ENTB

WRITE (ILUNIT, 2) WORD, ENTB

ENDIF

GOTO 300

C

IF (WORD .EQ. 'TBUMP') THEN

IF (INTOK .LT. 2) GOTO 777

TBUMP $=$ RELNUM $($ PWORD (2))

WRITE $(*, 2)$ WORD, TBUMP

WRITE (ILUNIT, 2) WORD, TBUMP GOTO 300

C

ENDIF

IF (WORD .EQ. 'TINTB') THEN

IF (INTOK .LT. 2) GOTO 777

TINTB $=$ RELNUM $($ PWORD $(2))$

WRITE $(*, 2)$ WORD, TINTB

WRITE (ILUNIT, 2) WORD, TINTB

GOTO 300

C

ENDIF

IF (WORD .EQ. 'TENDB') THEN

IF (INTOK . LT. 2) GOTO 777

TENDB $=$ RELNUM $(\operatorname{PWORD}(2))$

WRITE $(*, 2)$ WORD, TENDB

WRITE (ILUNIT, 2) WORD, TENDB

GOTO 300 


\section{RPP-6213 REV 0}

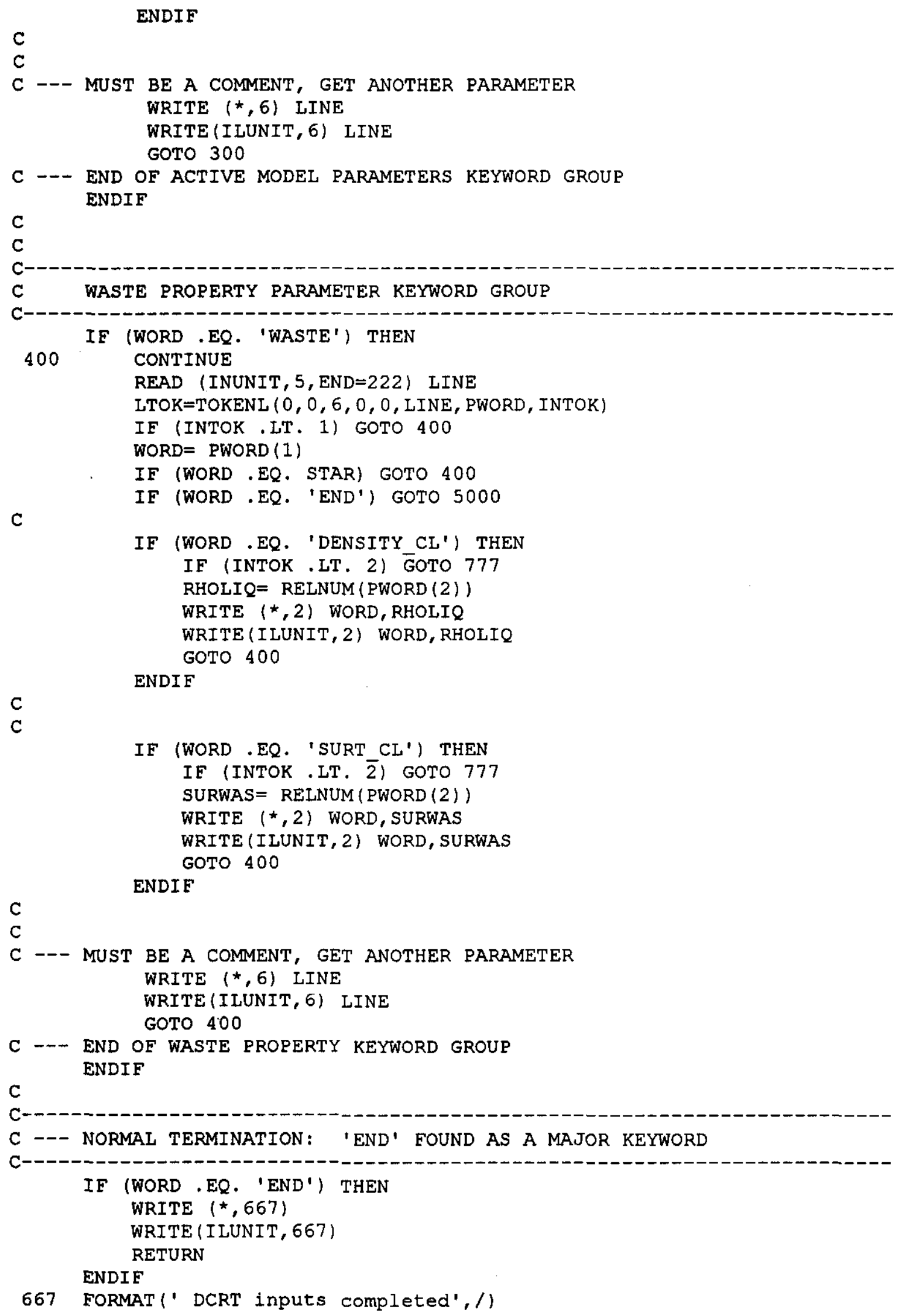

C

C

C

$\mathrm{C}$

C --- MUST BE A COMMENT, GET ANOTHER PARAMETER WRITE $(*, 6)$ LINE WRITE(ILUNIT, 6) LINE 


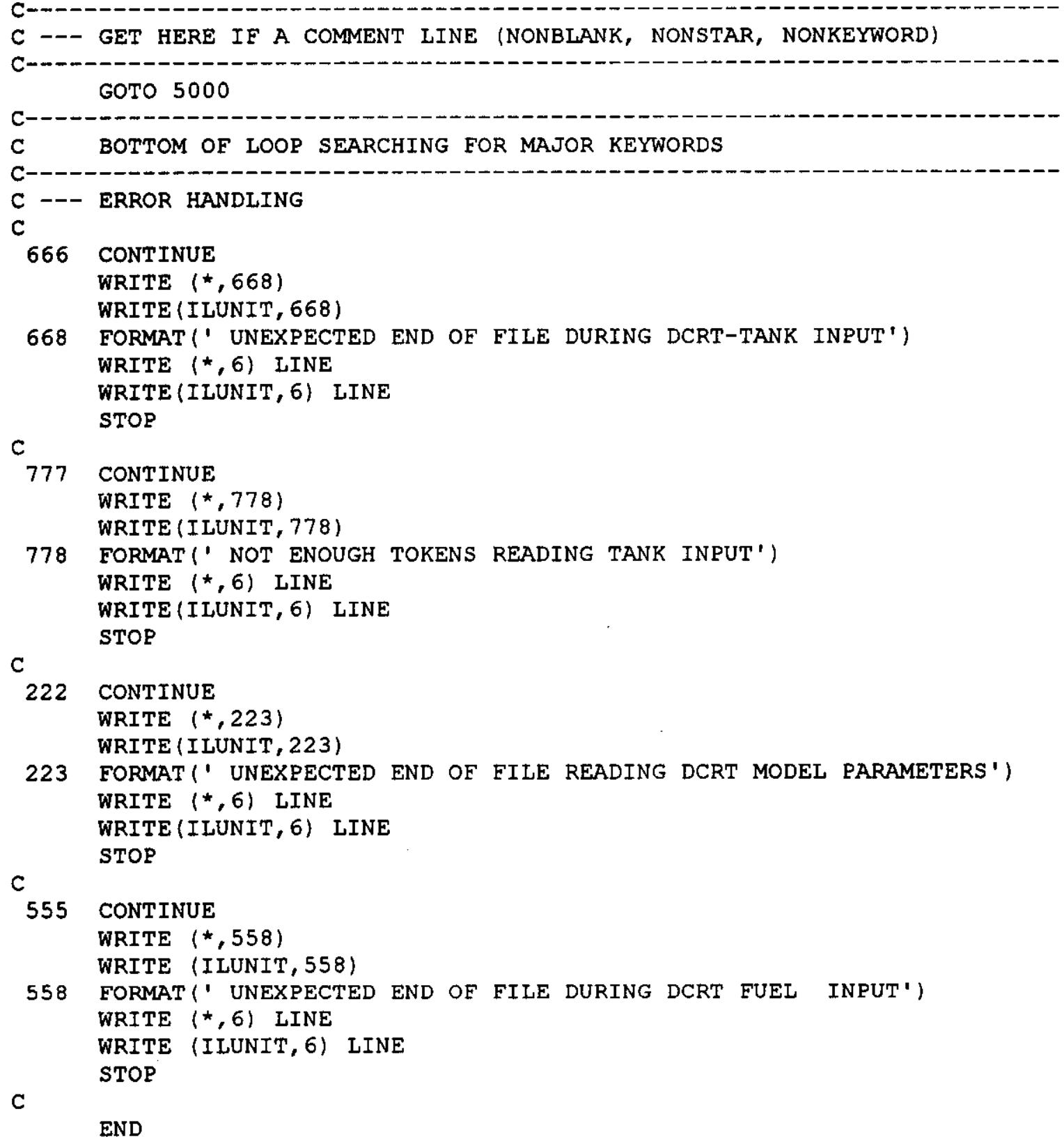

\section{F.2 BUMP.CML: Addition to HADCRT for Chapter 8}

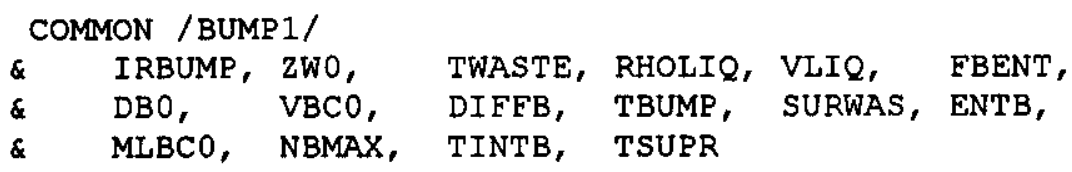

\section{F.3 Differences in AMIO.FOR: Addition to HADCRT for Chapter 8}

BeyondCompare Version 2.00 


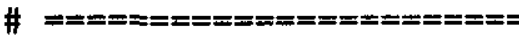

\# deleted

\#\#\#\#\#\#4186\#\#\#\#\#\#\#\#\#\#\#\#. . \code11 \amod. for

\section{F.5 Differences in CNTRL.CML: Addition to HADCRT for Chapter 8}

BeyondCompare Version 2.00

Copyright (C) stepping stone software 1987. All rights reserved.

Portions Copyright (C) Microsoft Corp 1984, 1985, 1986. All rights reserved.

\#\#\#\#\#00006\#\#\#\#\#\#\#\#\#\#\#\#cntrl.cml

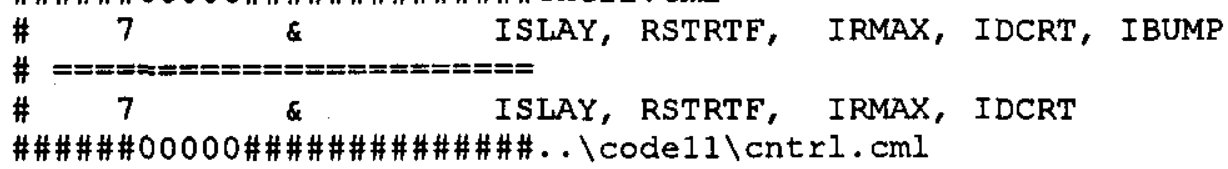

\section{F.6 AZ102.DAT: Input to HADCRT for Chapter 8}

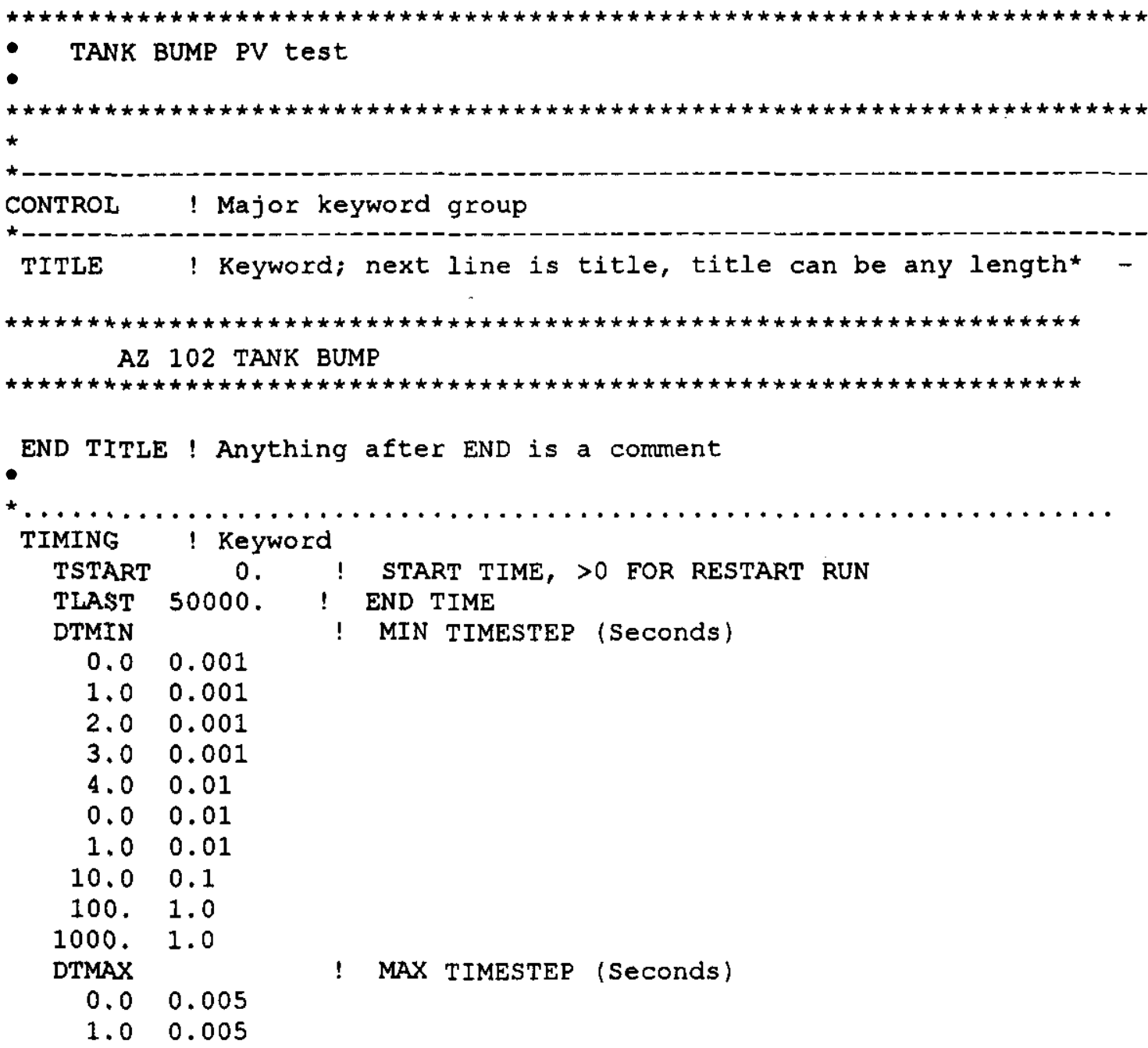




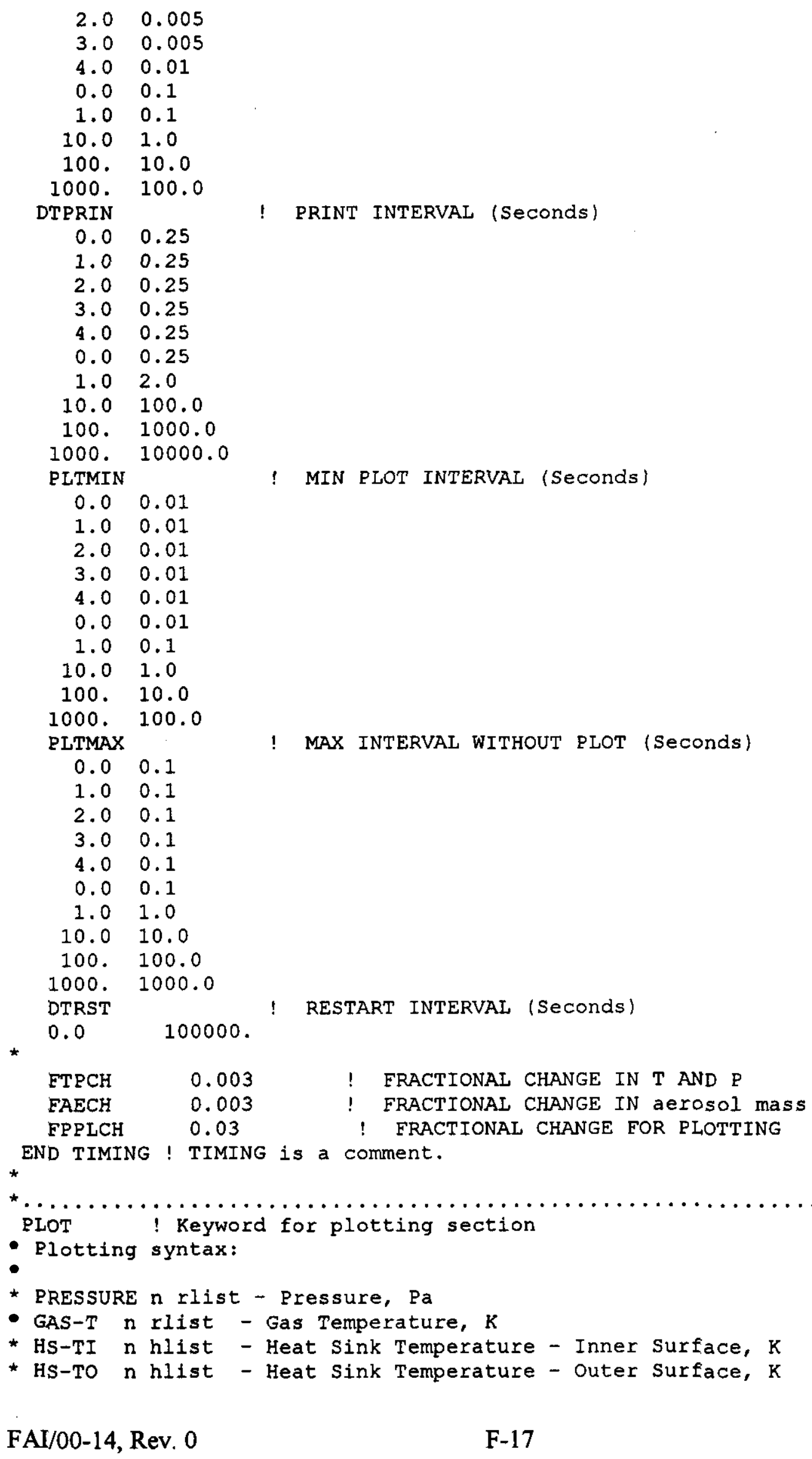




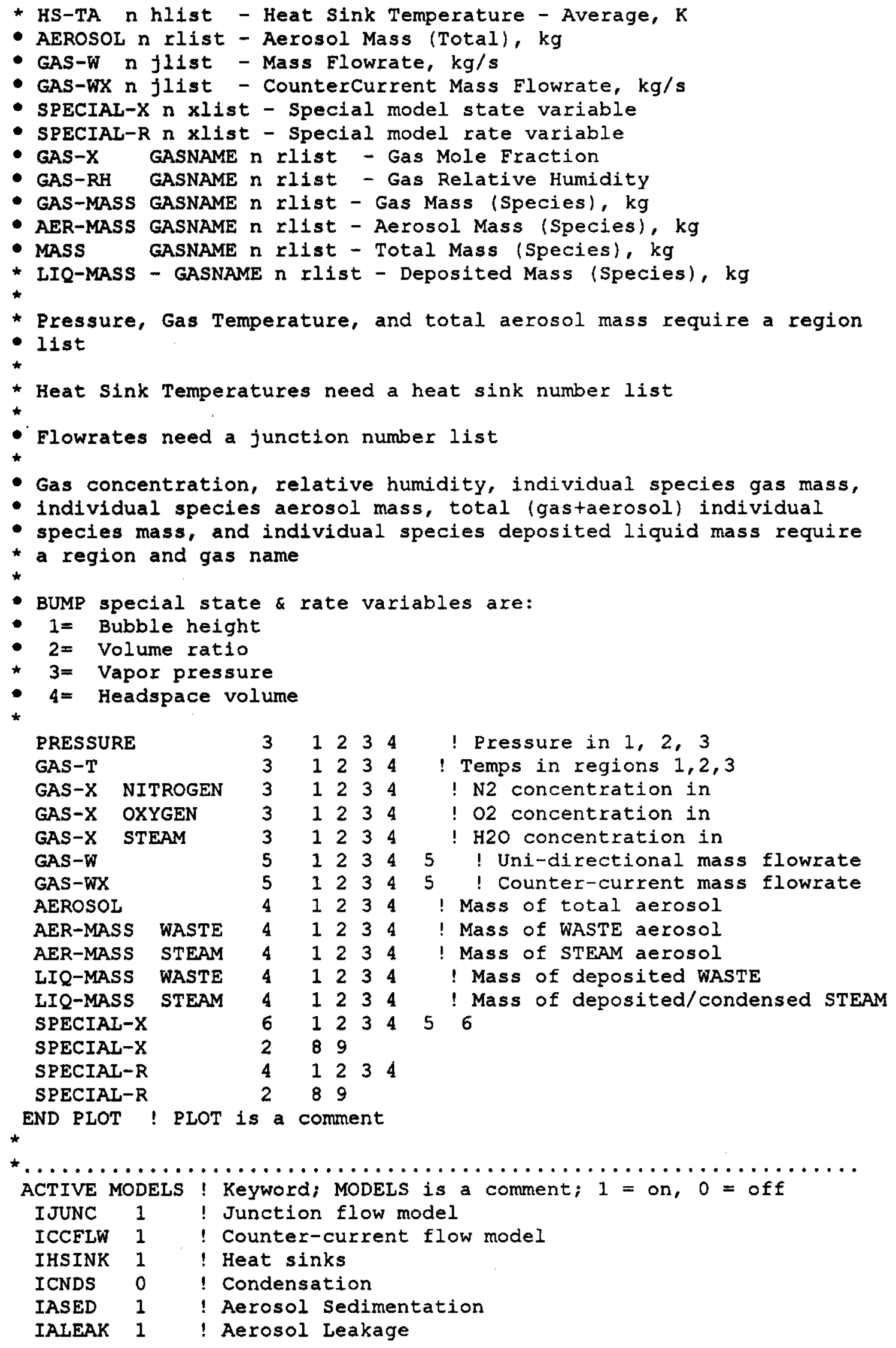




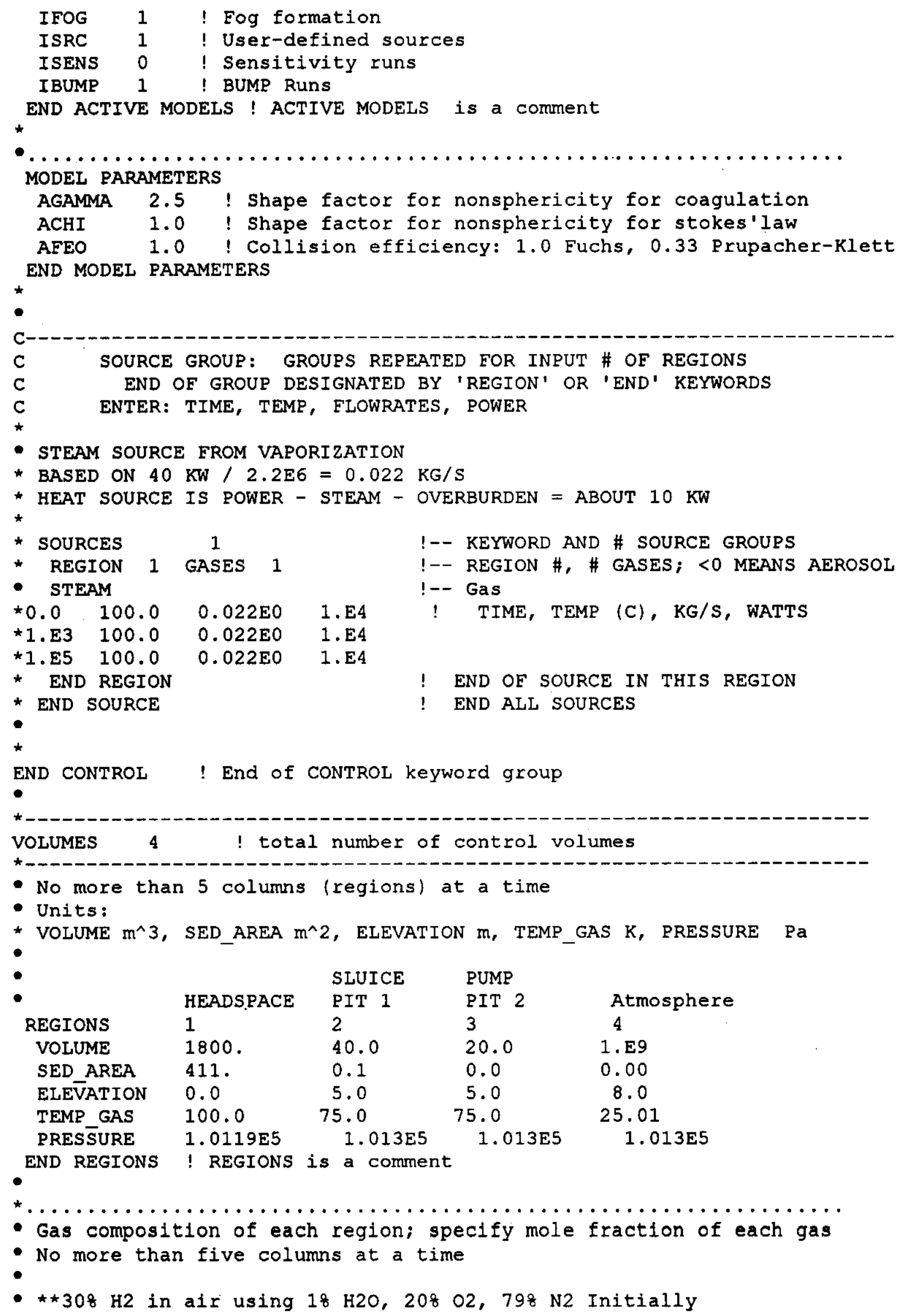




\section{RPP-6213 REV 0}

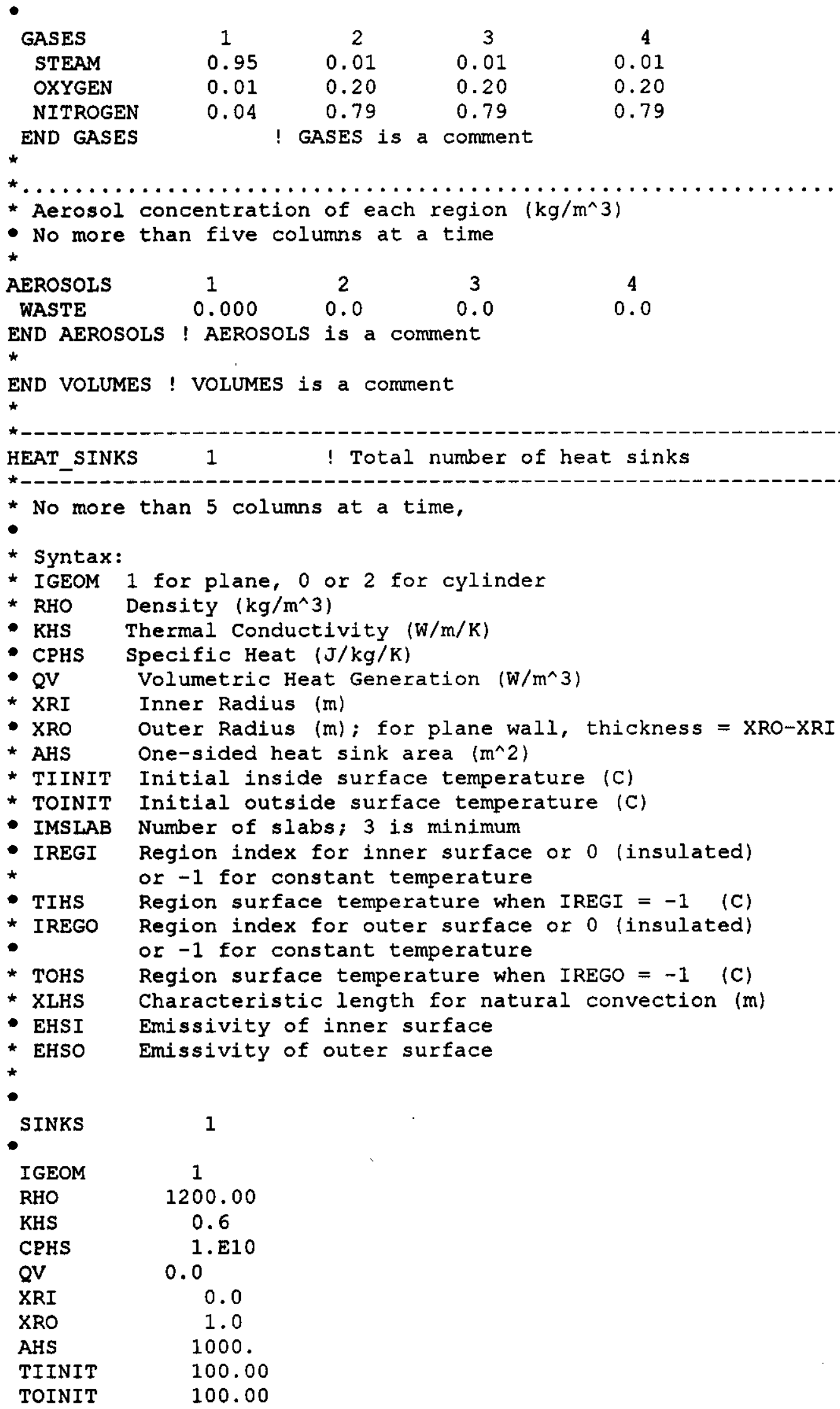




$\begin{array}{lc}\text { IMSLAB } & 10 \\ \text { IREGI } & 1 \\ \text { TIHS } & 100.0 \\ \text { IREGO } & 0 \\ \text { TOHS } & 100.0 \\ \text { XLHS } & 10.0 \\ \text { EHSI } & 1.0 \\ \text { EHSO } & 1.0 \\ \text { END } & \end{array}$

END HEAT_SINKS ! HEAT_SINKS is a comment

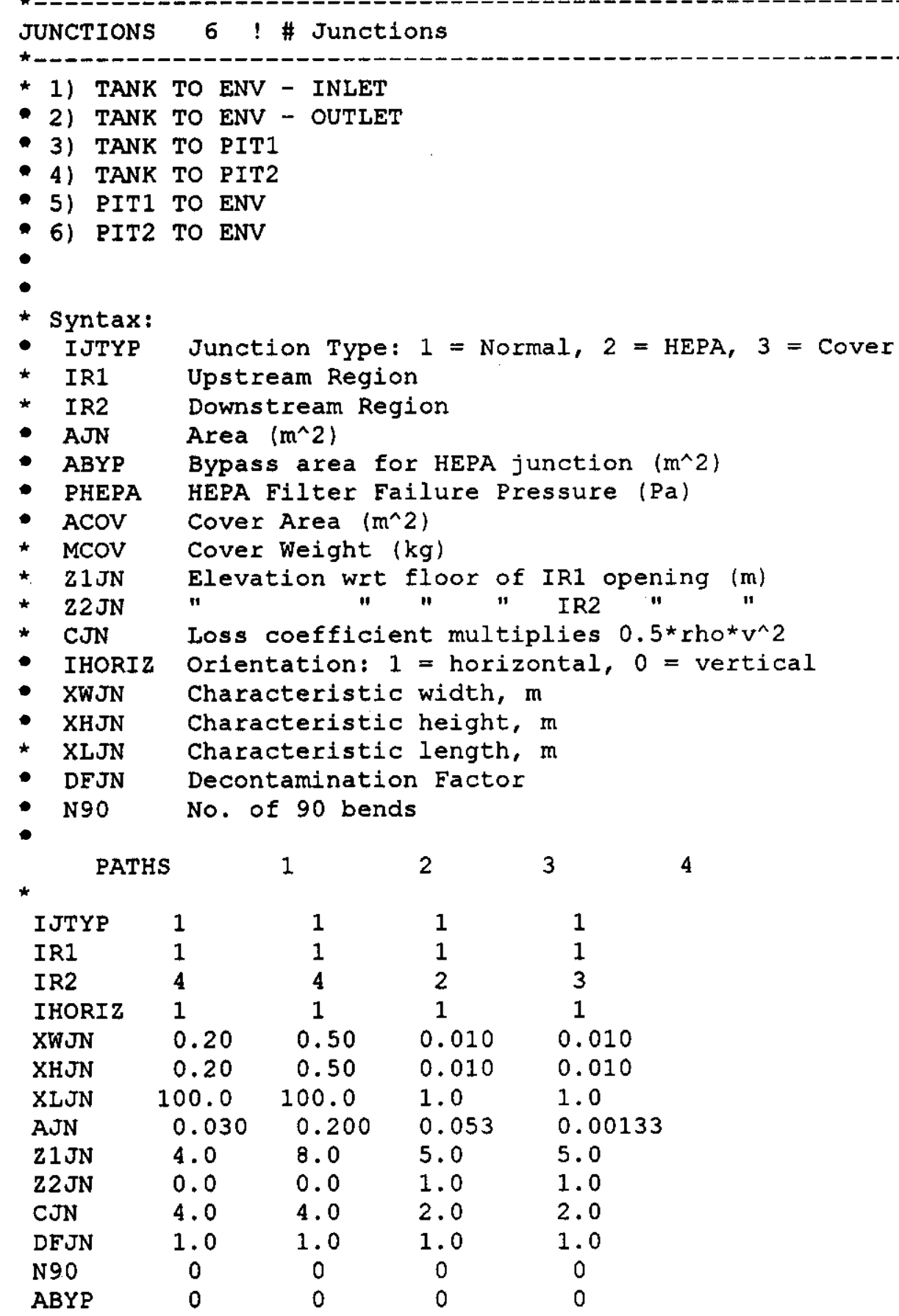




$\begin{array}{cccll}\text { PHEPA } & 0 & 0 & 0 & 0 \\ \text { ACOV } & 0 & 0 & 0 & 0 \\ \text { MCOV } & 0 & 0 & 0 & 0 \\ \text { END PATHS } & \text { ! PATHS is a comment. }\end{array}$

-

END PATHS ! PATHS is a comment.

\begin{tabular}{lll}
\multicolumn{2}{c}{ PATHS } & 5 \\
IJTYP & 3 & 3 \\
IR1 & 2 & 3 \\
IR2 & 4 & 4 \\
IHORIZ & 1 & 1 \\
XWJN & 0.01 & 0.01 \\
XHJN & 0.01 & 0.01 \\
XIJN & 0.3 & 0.3 \\
AJN & 0.32 & 0.08 \\
Z1JN & 0.6 & 0.6 \\
Z2JN & 0.0 & 0.0 \\
CJN & 2.0 & 2.0 \\
DFJN & 1.0 & 1.0 \\
N90 & 0 & 0 \\
DP1 & 0 & 0 \\
DP2 & 0 & 0 \\
ABYP & 0 & 0 \\
PHEPA & 0 & 0 \\
ACOV & 5.00 & 9.50 \\
MCOV & $6.8 \mathrm{E} 3$ & $16.6 \mathrm{E} 3$ \\
END & &
\end{tabular}

END PATHS ! PATHS is a comment.

$$
\text { * }
$$

END JUNCTIONS! JUNCTIONS is a comment.

$\bullet$

BUMP! Input section for "DCRT.For" Model

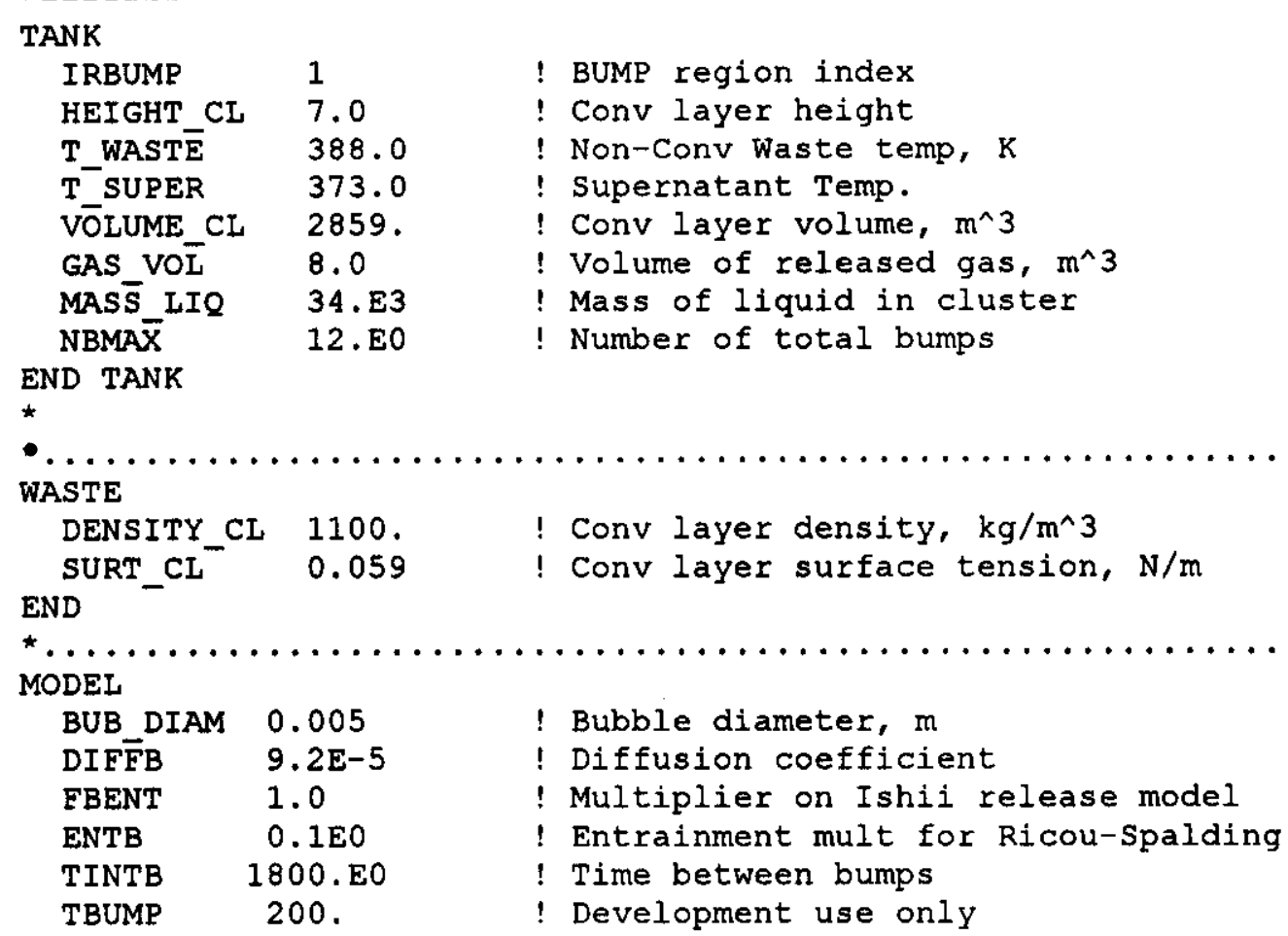


RPP-6213 REV 0

END MODEL

$\star$

-

END DCRT 
RPP-6213 REV 0

This page intentionally left blank.

FAI/00-14, Rev. 0 


\section{G.0 SPREADSHEETS FOR BUMP CRITERIA}

Time to Bump Conditions for Non-AZ/AY DSTs That Don't Make the Initial Screening Criteria: Current Waste Temperatures

Pedigreed Database Heat Loads, Hu et al. (2000) Inventories

\begin{tabular}{|c|c|c|c|c|c|c|c|c|c|c|c|}
\hline DST: & $\begin{array}{l}\text { RhoCL } \\
(\mathrm{kg} / \mathrm{m} 3)\end{array}$ & $\begin{array}{l}\text { RhoNCL } \\
(\mathbf{k} / \mathrm{m} 3)\end{array}$ & $\mathrm{T}(\mathrm{K})$ & $\operatorname{VCL}\left(\mathrm{m}^{3}\right)$ & VNCL $\left(m^{3}\right)$ & $\operatorname{CCL}(\mathrm{J} / \mathrm{kg})$ & $\begin{array}{l}\text { CNCL } \\
(\mathrm{J} / \mathrm{kg})\end{array}$ & $Q(W)$ & $\begin{array}{c}\mathbf{k} \\
(W / \mathbf{m}-K)\end{array}$ & dsoil, m & Rtank, m \\
\hline SY101 & 1390 & 1610 & 323 & 2286 & 2214 & 3000 & 3300 & 16100 & 1 & 4 & 11 \\
\hline AN103 & 1490 & 1710 & 314 & 2074 & 1552 & 3000 & 3300 & 18700 & 1 & 4 & 11 \\
\hline AN104 & 1400 & 1590 & 317 & 2286 & 1700 & 3000 & 3300 & 19700 & 1 & 4 & 11 \\
\hline AN105 & 1420 & 1580 & 313 & 2411 & 1851 & 3000 & 3300 & 13400 & 1 & 4 & 11 \\
\hline AW101 & 1400 & 1630 & 314 & 3104 & 1158 & 3000 & 3300 & 15200 & 1 & 4 & 11 \\
\hline AN102 & 1410 & 1500 & 320 & 3676 & 337 & 3000 & 3300 & 11500 & 1 & 4 & 11 \\
\hline AN107 & 1370 & 1560 & 308 & 3017 & 935 & 3000 & 3300 & 13700 & 1 & 4 & 11 \\
\hline
\end{tabular}

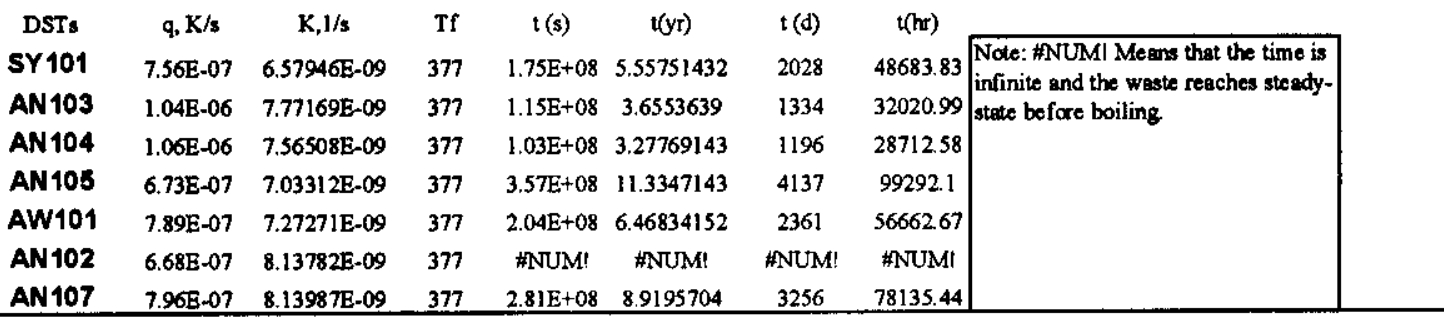

Hu et al. (2000) Heat Loads and Inventories

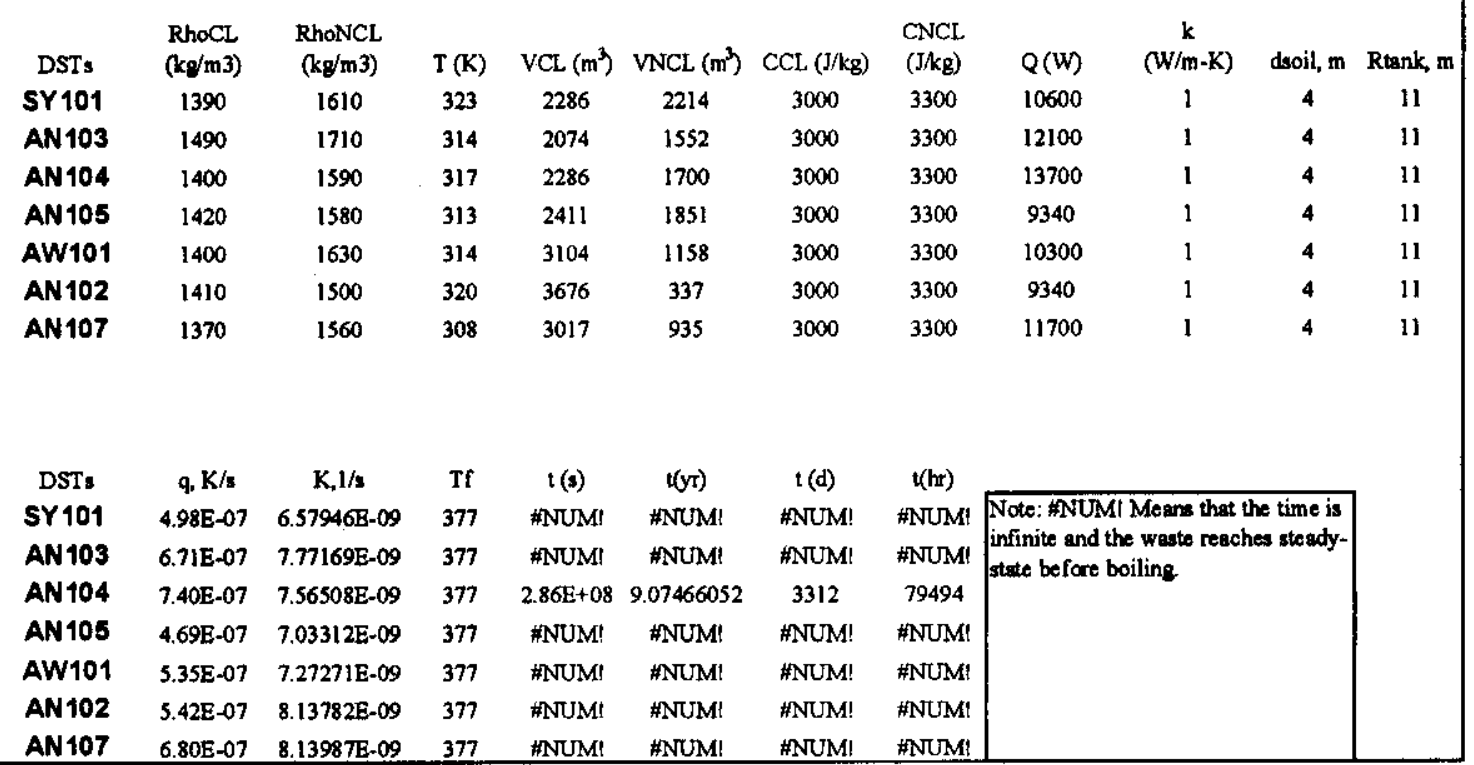


Time to Bump Conditions for Non-AZ/AY DSTs That Don't Make the Initial Screening Criteria: LCO Waste Temperatures

Pedigreed Database Heat Loads, Hu et al. (2000) Inventories

\begin{tabular}{|c|c|c|c|c|c|c|c|c|c|c|c|}
\hline DST, & $\begin{array}{l}\text { RhoCl } \\
(\mathbf{k g} / \mathrm{m} 3)\end{array}$ & $\begin{array}{c}\text { RhoNCL } \\
(\mathbf{k g} / \mathbf{m} 3)\end{array}$ & $T(K)$ & $\operatorname{VCL}\left(\mathrm{m}^{3}\right)$ & VNCL $\left(m^{3}\right)$ & $\operatorname{CCL}(\mathrm{J} / \mathrm{kg})$ & $\begin{array}{l}\mathrm{CNCL} \\
(\mathrm{J} / \mathbf{k g})\end{array}$ & $Q(W)$ & $\underset{(W / m-K)}{k}$ & dsoil, m & Rtank, m \\
\hline SY101 & 1390 & 1610 & 364 & 2286 & 2214 & 3000 & 3300 & 16100 & 1 & 4 & 11 \\
\hline AN103 & 1490 & 1710 & 364 & 2074 & 1552 & 3000 & 3300 & 18700 & 1 & 4 & 11 \\
\hline AN104 & 1400 & 1590 & 364 & 2286 & 1700 & 3000 & 3300 & 19700 & 1 & 4 & 11 \\
\hline AN108 & 1420 & 1580 & 364 & 2411 & 1851 & 3000 & 3300 & 13400 & 1 & 4 & 11 \\
\hline AW101 & 1400 & 1630 & 364 & 3104 & 1158 & 3000 & 3300 & 15200 & 1 & 4 & 11 \\
\hline AN102 & 1410 & 1500 & 364 & 3676 & 337 & 3000 & 3300 & 11500 & 1 & 4 & 11 \\
\hline AN107 & 1370 & 1560 & 364 & 3017 & 935 & 3000 & 3300 & 13700 & 1 & 4 & 11 \\
\hline
\end{tabular}

\begin{tabular}{|c|c|c|c|c|c|c|c|c|}
\hline DST: & $\mathrm{q}, \mathrm{K} / \mathbf{m}$ & K, $1 / \mathbf{s}$ & Tf & $t(s)$ & $t(y r)$ & $t$ (d) & $t(h r)$ & \\
\hline SY101 & $7.56 \mathrm{E}-07$ & 6.57946E-09 & 377 & 63833563 & 2.02414901 & 739 & 17731.5453 & \multirow{7}{*}{$\begin{array}{l}\text { Note: \#NUM! Means that the time is } \\
\text { infinite and the waste reaches steady- } \\
\text { state before boiling. }\end{array}$} \\
\hline AN103 & $1.04 \mathrm{E}-06$ & $7.77169 \mathrm{E}-09$ & 377 & 33670277 & 1.06767747 & 390 & 9352.85461 & \\
\hline AN104 & $1.06 \mathrm{E}-06$ & $7.56508 \mathrm{E}-09$ & 377 & 30225878 & 0.9584563 & 350 & 8396.0772 & \\
\hline AN105 & $6.73 \mathrm{E}-07$ & $7.03312 \mathrm{E}-09$ & 377 & $1.7 \mathrm{E}+08$ & 5.39160547 & 1968 & 47230.4639 & \\
\hline AW101 & $7.89 \mathrm{E}-07$ & $7.27271 \mathrm{E}-09$ & 377 & 73231063 & 2.32214178 & 848 & 20341.962 & \\
\hline AN102 & $6.68 \mathrm{E}-07$ & $8.13782 \mathrm{E}-09$ & 377 & \#NUM! & \#NUM! & \#NUM! & \#NUM! & \\
\hline AN107 & $7.96 \mathrm{E}-07$ & $8.13987 \mathrm{E}-09$ & 377 & $1.21 \mathrm{E}+08$ & 3.82788195 & 1397 & 33532.2459 & \\
\hline
\end{tabular}

Hu et al (2000) Heat Loads and Inventories

\begin{tabular}{|c|c|c|c|c|c|c|c|c|c|c|c|}
\hline DST: & $\begin{array}{l}\text { RhoCl } \\
(\mathbf{k g} / \mathrm{m} 3)\end{array}$ & $\begin{array}{l}\text { RhoNCL } \\
(\mathrm{kg} / \mathrm{m} \mathbf{3})\end{array}$ & $T(K)$ & $\operatorname{VCL}\left(\mathrm{m}^{3}\right)$ & $\operatorname{VNCL}\left(\mathrm{m}^{3}\right)$ & $\mathrm{CCL}(\mathrm{J} / \mathrm{kg})$ & $\begin{array}{l}\mathrm{CNCL} \\
(\mathrm{J} / \mathrm{kg})\end{array}$ & $Q(W)$ & $\underset{(W / m-K)}{\mathbf{k}}$ & dsoil, m & Rtank, m \\
\hline SY101 & 1390 & 1610 & 364 & 2286 & 2214 & 3000 & 3300 & 10600 & 1 & 4 & 11 \\
\hline AN103 & 1490 & 1710 & 364 & 2074 & 1552 & 3000 & 3300 & 12100 & 1 & 4 & 11 \\
\hline AN104 & 1400 & 1590 & 364 & 2286 & 1700 & 3000 & 3300 & 13700 & 1 & 4 & 11 \\
\hline AN106 & 1420 & 1580 & 364 & 2411 & 1851 & 3000 & 3300 & 9340 & 1 & 4 & 11 \\
\hline AW101 & 1400 & 1630 & 364 & 3104 & 1158 & 3000 & 3300 & 10300 & 1 & 4 & 11 \\
\hline AN102 & 1410 & 1500 & 364 & 3676 & 337 & 3000 & 3300 & 9340 & 1 & 4 & 11 \\
\hline AN107 & 1370 & 1560 & 364 & 3017 & 935 & 3000 & 3300 & 11700 & 1 & 4 & 11 \\
\hline
\end{tabular}

\begin{tabular}{|c|c|c|c|c|c|c|c|c|}
\hline DSTs & q, $K /:$ & $K, 1 / 8$ & Tf & $t(s)$ & $t(y r)$ & $t(d)$ & $t(h r)$ & \\
\hline SY101 & $4.98 \mathrm{E}-07$ & $6.57946 \mathrm{E}-09$ & 377 & $-3.6 \mathrm{E}+08$ & -11.400936 & -4161.3415 & -99872.195 & \multirow{7}{*}{$\begin{array}{l}\text { Note: \#NUM! Or negative values } \\
\text { means that the time is infinite and tho } \\
\text { waste reaches steady-state before } \\
\text { boiling. }\end{array}$} \\
\hline AN103 & $6.7 \mathrm{EE}-07$ & 7.77169E-09 & 377 & \#NUM! & \#NUM! & \#NUM! & \#NUM! & \\
\hline AN104 & $7.40 \mathrm{E}-07$ & $7.56508 \mathrm{E}-09$ & 377 & $1.3 \mathrm{E}+08$ & 4.11872159 & 1503 & 36080 & \\
\hline AN106 & 4.69E-07 & $7.03312 \mathrm{E}-09$ & 377 & $-1.16 E+08$ & -3.6707453 & -1339.822 & -32155.729 & \\
\hline AW101 & 5.35E-07 & $7.27271 \mathrm{E}-09$ & 377 & $-2.14 \mathrm{E}+08$ & -6.7723401 & -2471.9041 & -59325.699 & \\
\hline AN102 & $5.42 \mathrm{E}-07$ & $8.13782 \mathrm{E}-09$ & 377 & $-1 E+08$ & -3.172447 & -1157.9432 & -27790.636 & \\
\hline AN107 & $6.80 \mathrm{E}-07$ & $8.1398 \pi-09$ & 377 & \#NUM! & \#NUM! & \#NUM! & \#NUM! & \\
\hline
\end{tabular}




\begin{tabular}{|c|c|c|c|c|c|c|c|c|c|c|c|}
\hline \multicolumn{12}{|c|}{ Transient Temperature Rise Calculation for 241-AZ-101: Hu et al. (2000) Inventory } \\
\hline & $\begin{array}{l}\text { RhoCL } \\
\text { (ke/m3) }\end{array}$ & $\begin{array}{l}\text { RhoNCL } \\
(\mathbf{k g} / \mathrm{m} 3)\end{array}$ & $\mathrm{T}(\mathbf{K})$ & $\operatorname{VCL}\left(m^{3}\right)$ & $\operatorname{VNCL}\left(\mathrm{m}^{3}\right)$ & $\mathrm{CC}(\mathrm{J} / \mathrm{kg})$ & CCL $(J / k g)$ & CNCL $(\mathrm{J} / \mathrm{kg})$ & & & \\
\hline & 1190 & 1670 & 345 & 3021 & 178 & 3000 & 3300 & 3300 & & & \\
\hline & Tair & Tsoil & Aroe & htecoeff & $Q(W)$ & $\underset{(W / m \cdot K)}{k}$ & dsoil m & Rtenk, m & . & & \\
\hline & 285 & 287 & 411 & 5.187075872 & 77300 & I & 4 & 11 & & & \\
\hline Time & Dayn & TNCL & TCL & QCL. & QNCL & UPLOSS & QEX & DNLOSS & DTNCL & DTCL & DIFF \\
\hline 0 & 0.00 & 345.00 & 327.00 & 31351.0 & 45949.0 & 4315.5 & 38374.0 & 2167.1 & $5.51 \mathrm{E}-06$ & $5.51 \mathrm{E}-06$ & $-1.13 \mathrm{E}-04$ \\
\hline 500000 & 5.79 & 347.76 & 329.76 & 31351.0 & 45949.0 & 4598.8 & 38373.3 & 2270.1 & $5.41 \mathrm{E}-06$ & $5.49 \mathrm{~B}-06$ & \\
\hline 1000000 & 11.57 & 350.46 & 332.50 & 31351.0 & 45949.0 & 4880.8 & 38287.0 & 2371.1 & 5.39E- 06 & $5.46 \mathrm{E}-06$ & \\
\hline 1500000 & 17.36 & 353.16 & 335.23 & 31351.0 & 45949.0 & 5161.2 & 38217.8 & 2471.9 & $5.36 \mathrm{E}-06$ & $5.43 \mathrm{~B}-06$ & \\
\hline 2000000 & 23.15 & 355.84 & 337.95 & 31351.0 & 45949.0 & 5440.1 & 38145.7 & 25720 & $5.33 \mathrm{E}-06$ & $5.40 \mathrm{~B}-06$ & \\
\hline 2500000 & 28.94 & 358.50 & 340.65 & 31351.0 & 45949.0 & 5717.5 & 38074.6 & 2671.7 & $5.30 \mathrm{E}-06$ & $5.37 \mathrm{E}-06$ & \\
\hline 3000000 & 34.72 & 361.16 & 343.33 & 31351.0 & 45949.0 & 5993.4 & 38003.8 & 2770.8 & $5.27 \mathrm{E}-06$ & $5.34 \mathrm{E}-06$ & \\
\hline 3500000 & 40.51 & 363.79 & 346.00 & 31351.0 & 45949.0 & 6267.8 & 37933.4 & 2869.3 & $5.25 \mathrm{E}-\infty 6$ & $5.31 \mathrm{E}-06$ & \\
\hline 4000000 & 46.30 & 366.42 & 348.66 & 31351.0 & 45949.0 & 6540.7 & 37863.4 & 2967.3 & $5.22 \mathrm{E}-06$ & $5.28 \mathrm{E}-06$ & \\
\hline 4500000 & 52.08 & 369.03 & 351.30 & 31351.0 & 45949.0 & 68121 & 37793.8 & 3064.8 & $5.19 \mathrm{E}-06$ & $5.25 \mathrm{E}-06$ & \\
\hline 5000000 & 57.87 & 371.62 & 353.93 & 31351.0 & 45949.0 & 70820 & 37724.6 & 3161.7 & $5.16 \mathrm{E}-06$ & 5.23B-06 & \\
\hline 5500000 & 63.66 & 374.20 & 356.54 & 31351.0 & 45949.0 & 7350.5 & 37655.7 & 3258.1 & $5.13 \mathrm{E}-06$ & $5.20 \mathrm{E}-06$ & \\
\hline 6000000 & 69.44 & 376.77 & 359.14 & 31351.0 & 459.49 .0 & 7617.5 & 37587.2 & 3354.0 & $5.10 \mathrm{E}-06$ & $5.17 \mathrm{E}-06$ & \\
\hline 6500000 & 75.23 & 379.32 & 361.72 & 31351.0 & 45949,0 & 7883.1 & 37519.1 & 3449.4 & $5.08 \mathrm{~B}-06$ & $5.14 \mathrm{E}-06$ & \\
\hline 7000000 & 81.02 & 381.86 & 364.29 & 31351.0 & 45949.0 & 8147.2 & 37451.3 & 3544.3 & $5.05 \mathrm{E}-06$ & $5.11 \mathrm{~B}-06$ & \\
\hline 7500000 & 86.81 & 384.38 & 366.85 & 31351.0 & 45949.0 & 8409.8 & 37384.0 & 3638.6 & $5.028-06$ & $5.08 \mathrm{E}-06$ & \\
\hline 8000000 & 92.59 & 386.89 & 369.39 & 31351.0 & 459.49 .0 & 8671.1 & 37316.9 & 37324 & $4.99 \mathrm{E}-06$ & $5.06 \mathbb{E}-06$ & \\
\hline 8500000 & 98.38 & 389.39 & 371.92 & 31351.0 & 45949.0 & 8930.9 & 37250.3 & 3825.7 & $4.97 \mathrm{E}-06$ & $5.03 \mathrm{E}-06$ & \\
\hline 9000000 & 104.17 & 391.88 & 374.43 & 31351.0 & 45949.0 & 9189.3 & 37184.0 & 3918.5 & $4.94 \mathrm{E}-06$ & $5.00 \mathrm{E}-06$ & \\
\hline 9500000 & 109.95 & 394.35 & 376.93 & 31351.0 & 45949.0 & 9446.3 & 37118.1 & 4010.8 & 4.91E-06 & $4.98 \mathrm{E}-06$ & \\
\hline 9600000 & 111.11 & 394.84 & 377.43 & 31351.0 & 45949.0 & 9497.4 & 37105.0 & 4029.2 & 4.91E-06 & $4.97 \mathrm{E}-06$ & \\
\hline
\end{tabular}

Time is in seconds, TNCL is the non-convective layer temperature, TCL is the convective layer temperature, QCL is the heat load in the convective layer, QNCL is the heat load in the nonconvective layer, UPLOSS is the heat transfer rate in the upward direction, QEX is the exchange heat transfer between layers, DNLOSS is the downward heat transfer, DTNCL is the rate-ofchange $(\mathrm{K} / \mathrm{s})$ of the NCL temperature, and DTCL is the convective layer rate-of-change $(\mathrm{K} / \mathrm{s})$. 
RPP-6213 REV 0

Transient Temperature Rise Calculation for 241-AZ-102: Hu et al. (2000) Inventory and Pedigreed Database Feat Loads

\begin{tabular}{|c|c|c|c|c|c|c|c|c|c|c|c|}
\hline & $\begin{array}{l}\text { Rhool } \\
\text { (4tim3) }\end{array}$ & $\begin{array}{l}\text { RhoNat } \\
\left(\mathbf{k} / \mathbf{m}^{3}\right)\end{array}$ & $\mathbf{T}(\mathbf{K})$ & $\mathrm{VCl}\left(\mathrm{m}^{3}\right)$ & $\operatorname{VNCL}\left(\mathrm{m}^{2}\right)$ & $\mathrm{CC}(/ / \mathrm{kg})$ & $\alpha C(1 / \mathbb{R})$ & CNCL (//AB) & & & \\
\hline & 1100 & 1490 & $35 s$ & 3131 & 394 & 3000 & 3300 & 3300 & & & \\
\hline & Tir & Twoil & Are & htooeff & $Q(W)$ & $(\mathrm{W} / \mathrm{m}-\mathrm{K})$ & dooil, $m$ & Rtanik, m & & & \\
\hline & 285 & 287 & 411 & 2.009499985 & 63000 & 1 & 4 & 11 & & & \\
\hline Trine & Daye & TNCL & Ta & $Q C L$ & QNCL & UPLOSS & QEX & DNLOSS & DTNCL & DTC. & DIFF \\
\hline 0 & 0 & 355.00 & 321.00 & 24106.0 & 38894.0 & 3699.0 & 28080.8 & 2540.7 & $4.27 \mathrm{E}-06$ & 4.27E- -06 & 894E-04 \\
\hline 500000 & 5.787037007 & 357.14 & 323.13 & 24106.0 & 38894.0 & 3918.2 & 28082.3 & 2620.5 & $4.23 \mathrm{E}-06$ & 4.25E-06 & \\
\hline 1000000 & 1157407407 & 359.25 & 325.26 & 24106.0 & 38894.0 & 4136.4 & 28074.5 & 26995 & $4.19 \mathrm{E}-06$ & $4.23 \mathrm{E}-06$ & \\
\hline 1500000 & 1736111111 & 361.34 & 327.37 & 24106.0 & 38894.0 & 4353.5 & 28059.7 & $2 m s$ & $4.16 \mathrm{E}-06$ & $4.21 \mathrm{E}-06$ & \\
\hline 2000000 & 23.14814815 & 363.4 & 329.47 & 24106.0 & 38894.0 & 4569.7 & 28039.8 & 28555 & $4.13 \mathrm{E}-06$ & $4.19 \mathrm{E}-06$ & \\
\hline 2500000 & 2893518519 & 365.4 & 331.57 & 24106.0 & 38894.0 & 4784.7 & 28016.1 & 2932.6 & $4.10 E-06$ & $4.16 \mathrm{E}-06$ & \\
\hline 3000000 & 34.722222 & 367.54 & 333.65 & 24106.0 & 38894.0 & 4998.7 & 27989.7 & 30092 & $4.08 \mathrm{E}-06$ & 4.14E-06 & \\
\hline 3500000 & 4050925926 & 369.98 & 335.72 & 24106.0 & 38894.0 & 52116 & 27961.4 & 3085.4 & $4.058-06$ & 4.12E- -06 & \\
\hline 4000000 & 462962963 & 371.60 & 337.78 & 24106.0 & 38894.0 & 5423.4 & 27931.6 & 31610 & $4.03 \mathrm{E}-06$ & 4. $10 \mathrm{E}-06$ & \\
\hline 4500000 & 52.08033333 & 373.62 & 339.83 & 24106.0 & 38894,0 & 5634.1 & 27900.8 & 32363 & $4.00 \mathrm{E}-06$ & $4.088-06$ & \\
\hline 5000000 & 5787037007 & 375.62 & 341.87 & 24106.0 & 38894.0 & 5843.7 & 27869.3 & 3331.1 & $3998-06$ & 4.06E-06 & \\
\hline 5500000 & 63.65740741 & 377.61 & 343.90 & 24106.0 & 38894.0 & 6052.2 & 27837.4 & 3385,4 & $396 E-06$ & $4.04 E-06$ & \\
\hline 6000000 & 69,44444444 & 379.59 & 345.92 & 24106.0 & 38894.0 & 6259.7 & 27805.1 & 3459.4 & $394 \mathrm{E}-06$ & $4.02 E-06$ & \\
\hline 6500000 & 7523148148 & 381.56 & 347.93 & 24106.0 & 38894.0 & 6466.0 & $27 m 2.7$ & 3533.0 & $392 \mathrm{E}-06$ & $4.00 \mathrm{E}-06$ & \\
\hline 7000000 & 81.01851852 & 360.52 & 349.93 & 24106.0 & 38894.0 & 66713 & 27740.2 & 36062 & $390 \mathrm{E}-06$ & $397 \mathrm{E}-06$ & \\
\hline 7500000 & 8680655556 & 385.46 & 351.91 & 24106.0 & 38894.0 & 68755 & 27707.6 & 36789 & $3.88 \mathrm{E}-06$ & 3.95E-06 & \\
\hline 8000000 & 92.59259259 & 387.40 & 353.89 & 24106.0 & 38894.0 & 7078.6 & 27675.1 & 37513 & $3.85 \mathrm{E}-06$ & $3.93 \mathrm{E}-06$ & \\
\hline 8500000 & 9837962963 & 389.33 & 355.8 & 24106,0 & 38894.0 & 7280.7 & 27642.6 & 38233 & $3.83 E-06$ & $391 \mathrm{E}-06$ & \\
\hline 9000000 & 104.1666667 & 391.25 & 357.81 & 24106.0 & 38894.0 & 7481.7 & 27610.2 & 3895.0 & $381 \mathrm{E}-06$ & $389 \mathrm{E}-06$ & \\
\hline 9500000 & 109.9537037 & 393.15 & 359.76 & 24106.0 & 38894.0 & 7681.7 & 27578.0 & 3966.2 & $3.79 \mathrm{E}-06$ & $387 \mathrm{E}-06$ & \\
\hline 10000000 & 115.7407407 & 395.05 & $361 . \pi$ & 24106.0 & 38894.0 & 7880.6 & 27545.8 & 4037.1 & $3.77 \mathrm{E}-06$ & 385E-06 & \\
\hline 10100000 & $116.281 \% 1$ & 395.4 & 36208 & 24106.0 & 38894.0 & 7920.1 & 27539.4 & 40512 & $3.77 \mathrm{E}-06$ & $3.85 E-06$ & \\
\hline 10200000 & 118.0555556 & 395.80 & 362.47 & 24106.0 & 38894.0 & 7959.7 & 27533.1 & 40653 & $3.77 \mathrm{E}-06$ & $3.84 \mathrm{E}-06$ & \\
\hline
\end{tabular}

Time is in seconds, TNCL is the non-convective layer temperature, TCL is the convective layer temperature, QCL is the heat load in the convective layer, QNCL is the heat load in the nonconvective layer, UPLOSS is the heat transfer rate in the upward direction, QEX is the exchange heat transfer between layers, DNLOSS is the downward heat transfer, DTNCL is the rate-ofchange $(\mathrm{K} / \mathrm{s})$ of the NCL temperature, and DTCL is the convective layer rate-of-change $(\mathrm{K} / \mathrm{s})$. 
RPP-6213 REV 0

Transient Temperature Rise Calculation for 241-AZ-101: Hu et al. (2000) Inventory and Pedigreed Database

Heat Loads, L.CO Conditions

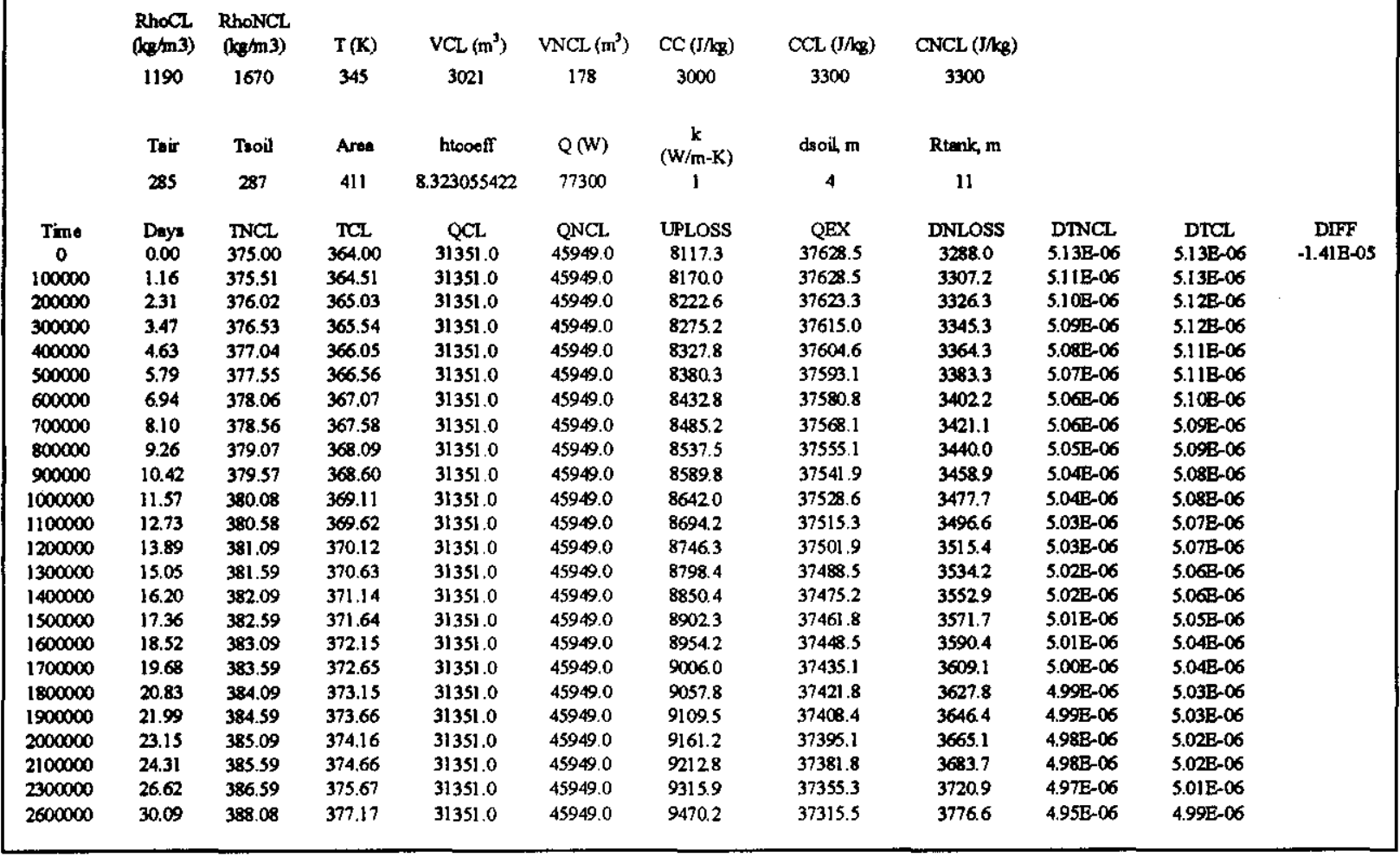

Time is in seconds, TNCL is the non-convective layer temperature, TCL is the convective layer temperature, QCL is the heat load in the convective layer, QNCL is the heat load in the nonconvective layer, UPLOSS is the heat transfer rate in the upward direction, QEX is the exchange heat transfer between layers, DNLOSS is the downward heat transfer, DTNCL is the rate-ofchange $(\mathrm{K} / \mathrm{s})$ of the NCL temperature, and DTCL is the convective layer rate-of-change $(\mathrm{K} / \mathrm{s})$. 


\begin{tabular}{|c|c|c|c|c|c|c|c|c|c|c|c|}
\hline \multicolumn{12}{|c|}{$\begin{array}{c}\text { Transient Temperature Rise Calculation for 241-AZ-102: Hu et al. (2000) Inventory and Pedigreed Database Heat Loads, } \\
\text { LCO Conditions }\end{array}$} \\
\hline & $\begin{array}{l}\text { Rhocl } \\
\left(\mathrm{k} / \mathrm{m}^{3}\right)\end{array}$ & $\begin{array}{l}\text { RhoNCL } \\
\text { (ke/m3) }\end{array}$ & $T(K)$ & $\mathrm{VCl}\left(\mathrm{m}^{3}\right)$ & VNCL $\left(m^{3}\right)$ & $\mathrm{CC}(5 / \mathrm{kg})$ & $\operatorname{CCL}(\mathrm{J} / \mathrm{kg})$ & $\mathrm{CNCL}(\mathrm{J} / \mathrm{kg})$ & & & \\
\hline & 1100 & 1490 & 355 & 3131 & 394 & 3000 & 3300 & 3300 & & & \\
\hline & Tair & Troil & Aron & hecosti & $Q(W)$ & $(w / m-K)$ & dooili, $m$ & $R(n k), m$ & & & \\
\hline & 2as & 287 & 411 & 6.212317934 & 63090 & 1 & 4 & 11 & & & \\
\hline Time & Daye & TNCI & TCL & $\propto$ L. & QNCL. & UPLOSS & QEX & DNLOSS & DTNCL & DTCL. & DFF \\
\hline 0 & 0 & 375.00 & 364.00 & 24106.0 & 38894.0 & 8117.3 & 28085.9 & 3238.0 & $3.00 \mathrm{E}-06$ & 3288E.06 & $9.62 E-04$ \\
\hline 100000 & 1.157407407 & 375.39 & ' $\quad 364.39$ & 24106.0 & 38894.0 & 8157.1 & 28086.8 & 3302.5 & $3.87 \mathrm{E}-06$ & 3.87E-06 & \\
\hline 200000 & 2.314834815 & 375.78 & 364.70 & 24106.0 & 38894.0 & 81969 & 28086.6 & 3317.0 & $3,87 \mathrm{E}-06$ & $3.87 \mathrm{E}-106$ & \\
\hline 300000 & 3.472222222 & 376.16 & 365.16 & 24106.0 & 38894.0 & 8236.7 & 28085.4 & $3331 \mathrm{~A}$ & $3.06 \mathrm{E}-06$ & $3.87 \mathrm{E}-06$ & \\
\hline 400000 & 4.62962963 & 376.35 & 365.35 & 24106.0 & 38894.0 & 8276.4 & 28083.4 & 3345.8 & 385E-06 & $3.86 \mathrm{~B}-06$ & \\
\hline 500000 & 5.747037037 & 376.93 & 365.94 & 24106.0 & 38894.0 & 8316.1 & 28080.7 & 33602 & $3.85 \mathrm{E}-06$ & 3.86E-06 & \\
\hline 600000 & 6.94444444 & 377.32 & 366.32 & 24106.0 & 38894.0 & 8355.8 & 28077.4 & 3374.6 & $3.44 \mathrm{E}-06$ & 3.06E-06 & \\
\hline 700000 & a.1010s1as2 & 377.70 & 366.71 & 24106.0 & 38894.0 & 8395.4 & 28073.6 & 3389.0 & $3.84 \mathrm{E}-06$ & 3.85E-06 & \\
\hline 800000 & 9.259299259 & 375.08 & 367.09 & 24106.0 & 38894.0 & 84.35 .0 & 28069.4 & 34033 & $3.83 \mathrm{E}-06$ & 3.25E-16 & \\
\hline 900000 & $10 A 1666667$ & 378.47 & 367,48 & 24106.0 & 38894.0 & 8474.5 & 28064.8 & 3417.6 & 3.00E-06 & 3.04E-06 & \\
\hline 1000000 & 11.57407407 & 378.85 & 367.26 & 24106.0 & 38894.0 & 8514.0 & 28060.0 & 3431.9 & $3.225-06$ & 3.2AE-06 & \\
\hline 1100000 & 12.73144148 & 379.23 & 368.25 & 24106.0 & 38894.0 & 8553.5 & 28054,9 & 3446.2 & $3.82 \mathrm{E}-06$ & 3.84E-A6 & \\
\hline 1300000 & 15.0462063 & 380.00 & 369.01 & 24106.0 & 38894.0 & 8632.3 & 28044.3 & 3474,7 & $3.81 \mathrm{E}-06$ & $3.93 \mathrm{E}-06$ & \\
\hline 1500000 & 1736111111 & 380.76 & 369.70 & 24106.0 & 38894.0 & 8711.0 & 28032.9 & 3503.1 & $3.00 \mathrm{E}-06$ & $3.285-06$ & \\
\hline 1700000 & 19.67592593 & 381.52 & 370.54 & 24106.0 & 38894.0 & 8789.5 & 28021.2 & 3531.5 & $3.79 \mathrm{E}-06$ & $3818-06$ & \\
\hline 1900000 & 2199074074 & 382.28 & 371.31 & 24106,0 & 38894.0 & 8867.9 & 28009.0 & 3559.8 & $3.75 \mathrm{E}-06$ & $3.818-06$ & \\
\hline 2100000 & 2430555556 & 383.03 & 372.07 & 24106.0 & 38894.0 & 8946.1 & $279 \% .7$ & 3588.1 & $3.77 \mathrm{E}-06$ & $3.80 \mathrm{E}-06$ & \\
\hline 2300000 & 26.62037037 & 383.79 & 372.83 & 24106.0 & 38894.0 & 9024.1 & 27984.3 & 3616.3 & $3.76 \mathrm{E}-06$ & $3.79 \mathrm{E}-06$ & \\
\hline 2500000 & 2093518519 & 384.54 & 373.58 & 24106.0 & 38894.0 & 9102.0 & 27971.8 & 36444 & $3.76 \mathrm{E}-06$ & $3.7 \mathrm{BE}-06$ & \\
\hline 2600000 & 30.09259259 & 384.91 & 373.96 & 24106.0 & 38894.0 & 9140.9 & 27965.5 & 3658.5 & $3.75 \mathrm{E}-06$ & $3.78 E-A 6$ & \\
\hline 2800000 & $324074074]$ & 385.67 & 374.72 & 24106.0 & 38894.0 & 9218.5 & 27952.9 & 3606.5 & $3.748-06$ & 3.77E-06 & \\
\hline 3000000 & $34.722212 \mathrm{m2}$ & $386.4 \mathrm{I}$ & 375.47 & 24106.0 & 38894.0 & 9295.9 & 27940.3 & 3714.5 & $3.74 E-06$ & $3.76 E-06$ & \\
\hline 3300000 & 38.19444444 & 387.54 & 376.60 & 24106.0 & 38894.0 & 9411.9 & 27921.3 & 3756.4 & $3.725-06$ & $3.75 \mathrm{E}-06$ & \\
\hline
\end{tabular}

Time is in seconds, TNCL is the non-convective layer temperature, TCL is the convective layer temperature, QCL is the heat load in the convective layer, QNCL is the heat load in the nonconvective layer, UPLOSS is the heat transfer rate in the upward direction, QEX is the exchange heat transfer between layers, DNLOSS is the downward heat transfer, DTNCL is the rate-ofchange $(\mathrm{K} / \mathrm{s})$ of the NCL temperature, and DTCL is the convective layer rate-of-change $(\mathrm{K} / \mathrm{s})$. 


\title{
H.0 SPREADSHEET FOR SCENARIO QUANTIFICATION
}

\section{H.1 BUMP FREQUENCY UNCERTAINTY ANALYSIS FOR THE CASE WITH NO VENTILATION CONTROLS}

\author{
Crystal Ball Report \\ Simulation started on 5/17/00 at 15:21:59 \\ Simulation stopped on $5 / 17 / 00$ at $15: 22: 47$
}

Forecast: Repair Failure Frequency

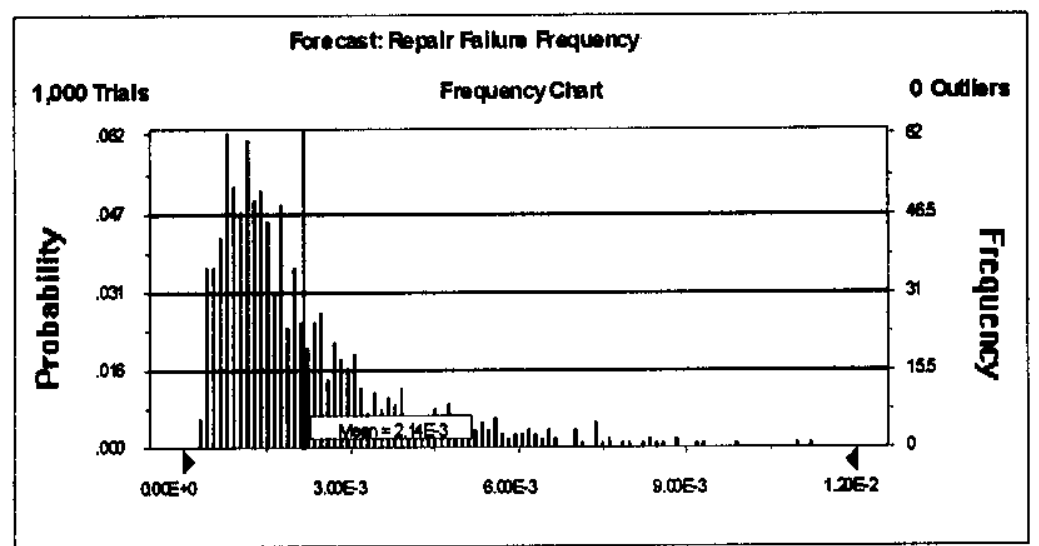

Percentiles:

$\begin{array}{ll}\text { Percentile } & \text { Value } \\ 0.0 \% & 2.30 \mathrm{E}-07 \\ 2.5 \% & 4.01 \mathrm{E}-07 \\ 5.0 \% & 4.62 \mathrm{E}-07 \\ 50.0 \% & 1.34 \mathrm{E}-06 \\ 95.0 \% & 3.74 \mathrm{E}-06 \\ 97.5 \% & 4.49 \mathrm{E}-06 \\ 100.0 \% & 6.84 \mathrm{E}-06\end{array}$

End of Forecast 
Forecast: Loss of HVAC Frequency $1 / y r$

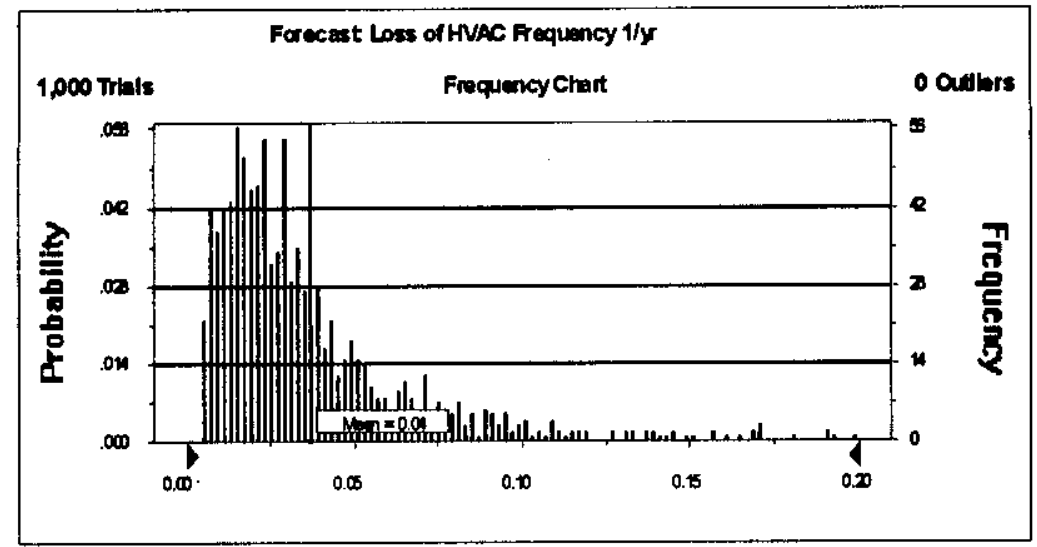

Percentiles:

$\begin{array}{ll}\text { Percentile } & \text { Value } \\ 0.0 \% & 0.00 \\ 2.5 \% & 0.01 \\ 5.0 \% & 0.01 \\ 50.0 \% & 0.03 \\ 95.0 \% & 0.10 \\ 97.5 \% & 0.13 \\ 100.0 \% & 0.20\end{array}$

End of Forecast 


\section{RPP-6213 REV 0}

\section{Forecast: Bump Frequeny 1/yr}

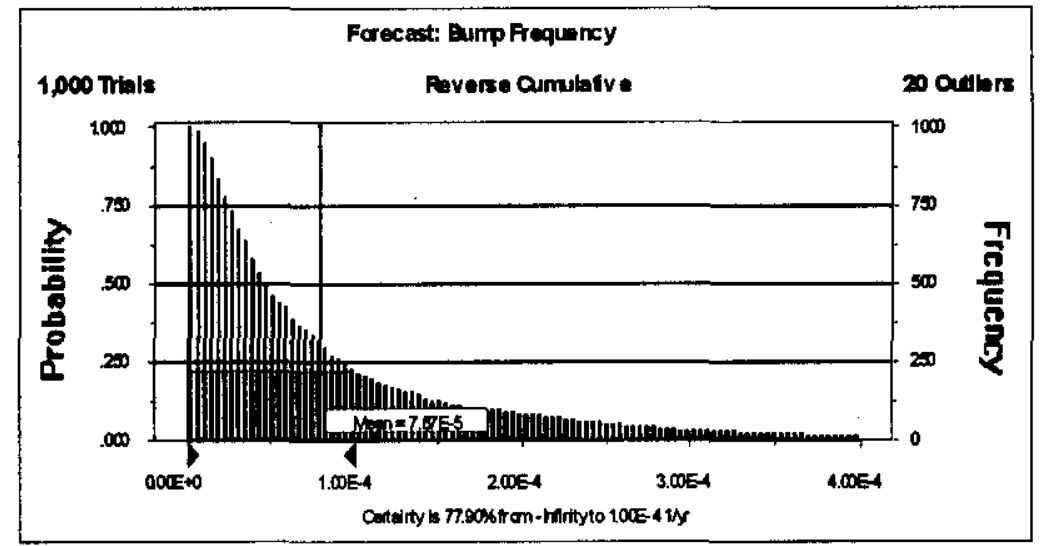

Percentiles:

\begin{tabular}{ll} 
Percentile & \multicolumn{1}{l}{ Value } \\
$0.0 \%$ & $2.00 \mathrm{E}-09$ \\
$2.5 \%$ & $4.88 \mathrm{E}-09$ \\
$5.0 \%$ & $7.19 \mathrm{E}-09$ \\
$50.0 \%$ & $3.72 \mathrm{E}-08$ \\
$95.0 \%$ & $1.90 \mathrm{E}-07$ \\
$97.5 \%$ & $2.62 \mathrm{E}-07$ \\
$100.0 \%$ & $6.63 \mathrm{E}-07$
\end{tabular}

End of Forecast 


\section{Assumptions}

\section{Assumption: X3}

Uniform distribution with parameters:

$\begin{array}{ll}\text { Minimum } & 0.03 \\ \text { Maximum } & 0.10\end{array}$

Mean value in simulation was 0.06

\section{Assumption: Time to Bump (days)}

Uniform distribution with parameters:

Minimum $\quad 80.00$

Maximum $\quad 100.00$

Mean value in simulation was 90.19

\section{Assumption: Time to Start (days)}

Uniform distribution with parameters:

$\begin{array}{ll}\text { Minimum } & 1.00 \\ \text { Maximum } & 10.00\end{array}$

Mean value in simulation was 5.48

\section{Assumption: CM Fraction}

Uniform distribution with parameters:
Minimum
0.10
Maximum
0.20

Mean value in simulation was 0.15 
Assumption: Loss of HVAC Frequency 1/yr

Lognormal distribution with parameters:

Log Mean

$-3.61$

Log Std. Dev. $\quad 0.78$

Selected range is from 0.00 to +Infinity

Mean value in simulation was 0.04

End of Assumptions 


\section{H.2 BUMP FREQUENCY UNCERTAINTY ANALYSIS FOR THE CURRENT WASTE}

\section{Crystal Ball Report}

Simulation started on 5/17/00 at 15:21:59

Simulation stopped on 5/17/00 at 15:22:47

\section{Forecast: Repair Failure Frequency}

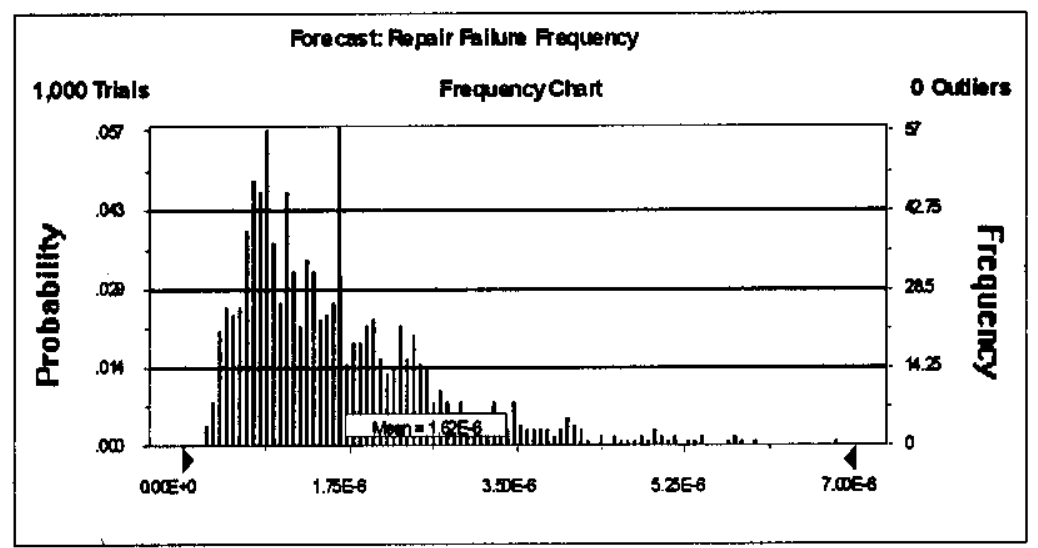

Percentiles:

\begin{tabular}{ll} 
Percentile & Value \\
\hline $0.0 \%$ & $2.30 \mathrm{E}-07$ \\
$2.5 \%$ & $4.01 \mathrm{E}-07$ \\
$5.0 \%$ & $4.62 \mathrm{E}-07$ \\
$50.0 \%$ & $1.34 \mathrm{E}-06$ \\
$95.0 \%$ & $3.74 \mathrm{E}-06$ \\
$97.5 \%$ & $4.49 \mathrm{E}-06$ \\
$100.0 \%$ & $6.84 \mathrm{E}-06$
\end{tabular}

End of Forecast 
Forecast: Loss of HVAC Frequency 1/yr

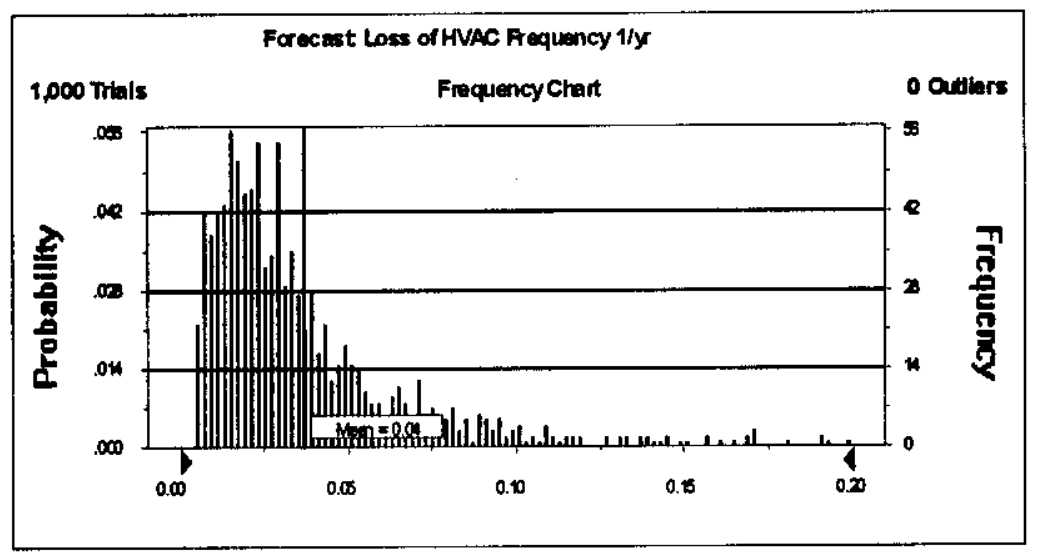

Percentiles:

\begin{tabular}{ll} 
Percentile & Value \\
\hline $0.0 \%$ & 0.00 \\
$2.5 \%$ & 0.01 \\
$5.0 \%$ & 0.01 \\
$50.0 \%$ & 0.03 \\
$95.0 \%$ & 0.10 \\
$97.5 \%$ & 0.13 \\
$100.0 \%$ & 0.20
\end{tabular}

End of Forecast 
RPP-6213 REV 0

Forecast: Bump Frequeny 1/yr

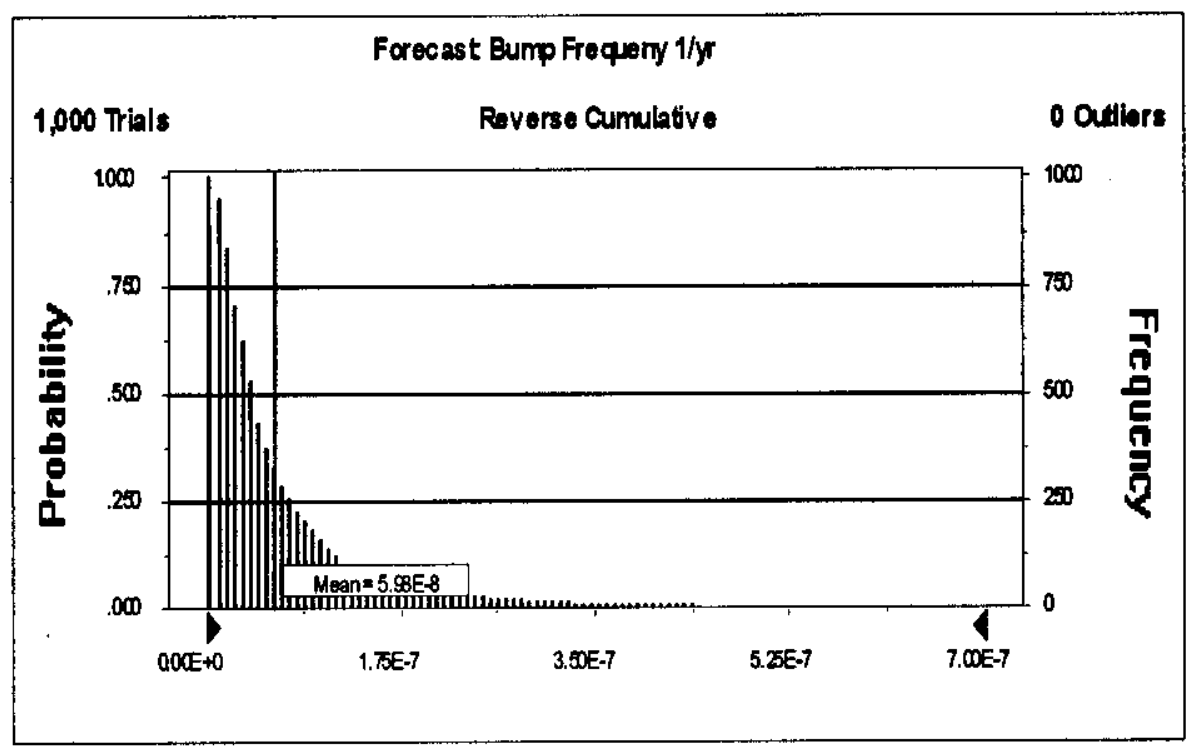

Percentiles:

$\begin{array}{ll}\text { Percentile } & \text { Value } \\ 0.0 \% & 2.00 \mathrm{E}-09 \\ 2.5 \% & 4.88 \mathrm{E}-09 \\ 5.0 \% & 7.19 \mathrm{E}-09 \\ 50.0 \% & 3.72 \mathrm{E}-08 \\ 95.0 \% & 1.90 \mathrm{E}-07 \\ 97.5 \% & 2.62 \mathrm{E}-07 \\ 100.0 \% & 6.63 \mathrm{E}-07\end{array}$

End of Forecast 


\section{Assumptions}

\section{Assumption: X3}

Uniform distribution with parameters:

$\begin{array}{ll}\text { Minimum } & 0.03 \\ \text { Maximum } & 0.10\end{array}$

Mean value in simulation was 0.06

\section{Assumption: Time to Bump (days)}

Uniform distribution with parameters:

$\begin{array}{ll}\text { Minimum } & 80.00 \\ \text { Maximum } & 100.00\end{array}$

Mean value in simulation was 90.19

\section{Assumption: Time to Start (days)}

Uniform distribution with parameters:

$\begin{array}{ll}\text { Minimum } & 1.00 \\ \text { Maximum } & 10.00\end{array}$

Mean value in simulation was 5.48

\section{Assumption: CM Fraction}

Uniform distribution with parameters:
Minimum
0.10
Maximum
0.20

Mean value in simulation was 0.15 
Assumption: Loss of HVAC Frequency $1 / \mathbf{y r}$

Lognormal distribution with parameters:

Log Mean

Log Std. Dev. $\quad 0.78$

Selected range is from 0.00 to +Infinity

Mean value in simulation was 0.04

\section{End of Assumptions}




\section{H.3 BUMP FREQUENCY UNCERTAINTY ANALYSIS FOR SEISMIC EVENTS}

\section{Crystal Ball Report}

Simulation started on $6 / 1 / 00$ at $17: 23: 38$

Simulation stopped on $6 / 1 / 00$ at $17: 36: 37$

Forecast: Tank Bump Frequency, Seismic [seismicfrequency.xls]integrate clipped normal

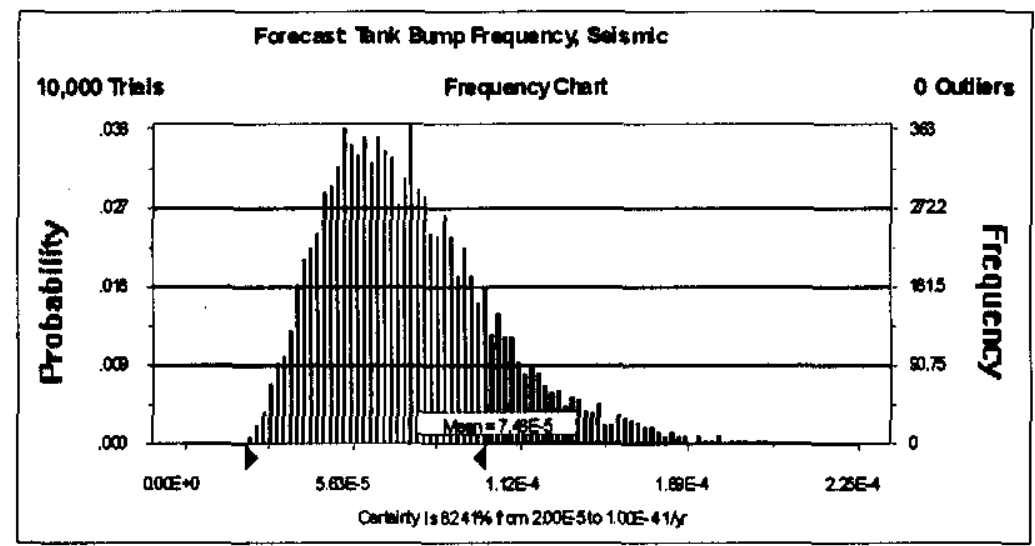

Percentiles:

Percentile

$0.0 \%$

$2.5 \%$

$5.0 \%$

$50.0 \%$

$95.0 \%$

$97.5 \%$

$100.0 \%$ $\underline{1 / \mathbf{y r}}$

2.03E-05

3.18E-05

3.65E-05

6.98E-05

1.30E-04

$1.45 \mathrm{E}-04$

2.08E-04

End of Forecast 


\section{RPP-6213 REV 0}

\section{Assumptions}

Assumption: mu

[seismicfrequency.xls]integrate clipped normal

Uniform distribution with parameters:

$\begin{array}{ll}\text { Minimum } & 0.40 \\ \text { Maximum } & 0.60\end{array}$

Mean value in simulation was 0.50

Assumption: $s \quad$ [seismicfrequency.xls]integrate clipped normal

Uniform distribution with parameters:

$\begin{array}{ll}\text { Minimum } & 5.00 \mathrm{E}-02 \\ \text { Maximum } & 1.00 \mathrm{E}-01\end{array}$

Mean value in simulation was $7.49 \mathrm{E}-2$

Assumption: $a^{\star} b \quad$ [seismicfrequency.xls]integrate clipped normal

Triangular distribution with parameters:

Minimum

$1.00 \mathrm{E}-05$

Likeliest

$2.26 \mathrm{E}-05$

Maximum

$5.00 \mathrm{E}-05$

Selected range is from $1.00 \mathrm{E}-5$ to $5.00 \mathrm{E}-5$

Mean value in simulation was $2.75 \mathrm{E}-5$ 


\section{I.0 QUALITY ASSURANCE DOCUMENTS}

Included here are:

1. Calculation Note Cover Sheet

2. Authorship and Reviewer Table

3. Calculation Note Methodology Checklist 
FAUSKE \& ASSOCIATES, INC.

\section{CALCULATION NOTE COVER SHEET}

\section{SECTION TO BE COMPLETED BY AUTHOR(S):}

Page I-2

Calc-Note Number FAI/00-14

Revision Number

0

Title __ Hanford Waste Tank Bump Accident and Consequence Analysis

Project No. or

Project _. Nuclear Safety \& Licensing Shop Order

CHGOIB

Purpose: Re-evaluate Hanford waste tank bump accident.

Results Summary: Physical models, criteria, frequency, and consequences are provided.

References of Resulting Reports, Letters, or Memoranda (Optional)

Author(s):

Name (Print or Type)

Michael Epstein

Boro Malinovic

Martin G. Plys

George Hauser
Signature

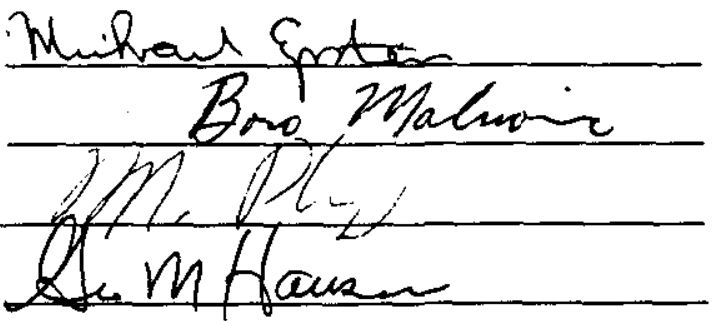

Completion Date 5131100 $5 / 31 / 20$ $5 / 3 / 62$ $5 / 3 / 100$

\section{SECTION TO BE COMPLETED BY VERIFIER(S):}

Verifier(s):
Name (Print or Type)
Martin G. Plys
Bethod of Verification: Design Review _. Alinovic

\section{SECTION TO BE COMPLETED BY MANAGER:}

Responsible Manager:

Name (Print or Type)

Martin G. Plys

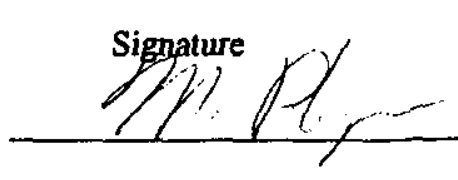

Approval

Date 


\begin{tabular}{|c|c|c|}
\hline Section & Author(s) & Reviewer(s) \\
\hline 1 & M. G. Plys & B. Malinovic \\
2 & M. G. Plys & B. Malinovic \\
3 & B. Malinovic & M. G. Plys \\
4 & M. Epstein & M. G. Plys \\
5 & M. Epstein & M. G. Plys \\
6 & B. Malinovic & M. G. Plys \\
7 & B. Malinovic & M. G. Plys \\
8 & M. G. Plys & B. Malinovic \\
9.1 & M. Epstein & M. G. Plys \\
9.2 & B. Malinovic & M. G. Plys \\
9.3 & M. G. Plys & B. Malinovic \\
10 & B. Malinovic & M. G. Plys \\
App. A & M. G. Plys & B. Malinovic \\
App. B & M. Epstein & M. G. Plys \\
App. C & M. Epstein & M. G. Plys \\
App. D & G. M. Hauser & M. G. Plys \\
App. E & G. M. Hauser & M. G. Plys \\
App. F & M. G. Plys & B. Malinovic \\
App. G & B. Malinovic & M. G. Plys \\
App. H & B. Malinovic & N/A \\
App. I & N/A & N/A \\
App. J & N/A & \\
\hline
\end{tabular}




\title{
CALCULATION NOTE METHODOLOGY CHECKLIST
}

\author{
CHECKLIST TO BE COMPLETED BY AUTHOR(S)
}

(CIRCLE APPROPRIATE RESPONSE)

1. Is the subject and/or the purpose of the design analysis clearly stated?.. YES - NO

2. Are the required inputs and their sources provided? YES $\bullet N O \bullet N / A$

3. Are the assumptions clearly identified and justified? YES $\bullet$ NO $\bullet$ N/A

4. Are the methods and units clearly identified? YES $\bullet N O \bullet N / A$

5. Have the limits of applicability been identified? YES • NO $\bullet N / A$ (Is the analysis for a 3 or 4 loop plant or for a single application.)

6. Are the results of literature searches, if conducted, or other background data provided? YES $\bullet N O \bullet N / A$

7. Are all the pages sequentially numbered and identified by the calculation note number? YES • NO

8. Is the project or shop order clearly identified? YES - NO

9. Has the required computer calculation information been provided? YES $\bullet N O \bullet N / A$

10. Were the computer codes used under configuration control?... YES $\bullet N O \bullet N / A$

11. Were the computer code(s) used applicable for modeling the physical and/or computational problems identified?.. YES • NO $\bullet N / A$ (Is the correct computer code being used for the intended purpose.)

12. Are the results and conclusions clearly stated? YES • NO

13. Are Open Items properly identified YES $\bullet$ NO $\bullet$ N/A

14. Were approved Design Control practices followed without exception? YES • NO $\bullet \mathrm{N} / \mathrm{A}$ (Approved Design Control practices refers to guidance documents within NSD that state how the work is to be performed, such as how to perform a LOCA analysis.)

15. Have all related contract requirements been met? YES • NO $\bullet$ N/A

NOTE: If NO to any of the above, Page Number containing justification: 
RPP-6213 REV 0

J.0 CHECKLIST FOR TECHNICAL PEER REVIEW 


\section{CHECKLIST FOR TECHNICAL PEER REVIEW}

Document Reviewed: RPP-6213

Scope of Review: The review consisted participation in a series of several meeting in which FAI presented their work and a review of the subject report. Items identified as NA below were not included in this review.

Yes No NA

[] [ ] [ $\mathrm{x}$ ] Previous reviews are complete and cover the analysis, up to the scope of this review, with no gaps.

[x] [] [] Problem is completely defined.

[x] [] [] Accident scenarios are developed in a clear and logical manner.

[x] [] [] Necessary assumptions are explicitly stated and supported.

[x] [] [] Computer codes and data files are documented.

[x] [] [] Data used in calculations are explicitly stated.

[] [] [x] Data were checked for consistency with original source information as applicable.

[] [] [x] Mathematical derivations were checked including dimensional consistency of results.

$[x]^{1}[][] \quad$ Models are appropriate and were used within their established range of validity or adequate justification was provided for use outside their established range of validity.

[ ] [ ] [ $\mathbf{x}$ ] Spreadsheet results and all hand calculations were verified.

[] [] [x] Software input is correct and consistent with the document reviewed.

[] [] [ $x$ ] Software output is consistent with the input and with the results reported in the document reviewed.

[ $x$ ] [ ] [ ] Limits/criteria/guidelines applied to the analysis results are appropriate and referenced. Limits/criteria/guidelines were checked against references.

[] [ ] [x ] Safety margins are consistent with good engineering practices.

[x ] [] [] Conclusions are consistent with analytical results and applicable limits.

[ $x]$ [ ] [ ] Results and conclusions address all points in the purpose.

[] [] [x] The document was prepared in accordance with HNF-2353, Section 4.3, Attachment B, "Calculation Note Format and Preparation Instructions".

\section{[x]【|【] Concurrence}

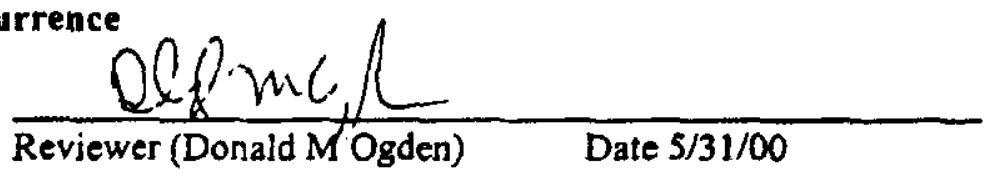

\footnotetext{
'Reviews conducted by John Marvin. Inc JMI) identified some areas of concern relative to the applicability of the models. A consensus was not reached. The JMI RCR comments document areas of disagreement.
} 


\section{CHECKLIST FOR TECHNICAL PEER REVITW}

Document Reviewed: RPPG2/3, HANFORD UASTE TAMK BOMP ACCIOENT AND CONSEQUENCE AUACYSIS. Scope of Review: TECHAXCAC BASIS FOR MODECS wOR STEAM DUMP Yes No NA

[x] [] [] Previous reviews are complete and cover the analysis, up to the scope of this review, with no gaps.

[x] [] [] Problem is completely defined.

[x] [] [] Accident scenarios are developed in a clear and logical manner.

[x] [] [] Necessary assumptions are explicitly stated and supported.

[] [ ] [x] Computer codes and data files are documented.

[x] [] [] Data used in calculations are explicitly stated.

[x] [] [] Data were checked for consistency with original source information as applicable.

[x] [] [] Mathematical derivations were checked including dimensional consistency of results.

[x] [ ] [ ] Models are appropriate and were used within their established range of validity or adequate justification was provided for use outside their established range of validity.

[ ] [ ] [ $x$ ] Spreadsheet results and all hand calculations were verified.

[] [] [x] Software input is correct and consistent with the document reviewed.

[] [] [x] Software output is consistent with the input and with the results reported in the document reviewed.

[ ] [ ] [x] Limits/criteria/guidelines applied to the analysis results are appropriate and referenced. Limits/criteria/guidelines were checked against references.

[] [] [ $x$ ] Safety margins are consistent with good engineering practices.

[x] [] [ ] Conclusions are consistent with analytical results and applicable limits.

[x] [ ] [ ] Results and conclusions address all points in the purpose.

[] [ ] The document was prepared in accordance with HNF-2353, Section 4.3, Attachment B, "Calculation Note Format and Preparation Instructions".

\section{[X] | ] [] Concurrence}

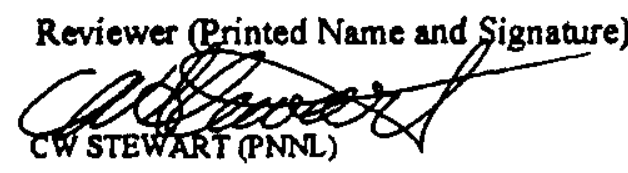

Date

$5 / 18100$ 
Preparation and Review of Calculation Notes
Manual

Desk Instruction

Page

Effective Date
HNF-2353

4.3, Rev, 0

8 of 8

June 10, 1999

\section{CHECKLIST FOR TECHNICAL PEER REVIEW}

Document Reviewed: FAI/OO-14. HaNFORD WASTE TaNk Bump Scope of Review: SECTION 8.4,... RADIOLAGICAL AND TOXIO

Yes No NA

$D$ [I] []

$\Delta[][]$

A [ [ []

$\Delta[][]$

[] [] $\mathbb{X}$

M [] []

Q [ [] []

(4) [] []

$\Delta[][]$

$x[][]$

[] [] $\infty$

[] [] $\infty$

$\otimes[][]$

$\Delta$ [] []

$\Delta$ [] []

$\Delta[][]$

$\Delta[]$ CHEmichl CONSEQUNCES

Previous reviews are complete and cover the analysis, up to the scope of this review, with no gaps.

Problem is completely defined.

Accident scenarios are developed in a clear and logical manner.

Necessary assumptions are explicitly stated and supported.

Computer codes and data files are documented.

Data used in calculations are explicitly stated.

Data were checked for consistency with original source information as applicable.

Mathematical derivations were checked including dimensional consistency of results.

Models are appropriate and were used within their established range of validity or adequate justification was provided for use outside their established range of validity.

Spreadsheet results and all hand calculations were verified.

Software input is correct and consistent with the document reviewed.

Software output is consistent with the input and with the results reported in the document reviewed.

Limits/criteria/guidelines applied to the analysis results are appropriate and referenced. Limits/criteria/guidelines were checked against references.

Safety margins are consistent with good engineering practices.

Conclusions are consistent with analytical results and applicable limits.

Results and conclusions address all points in the purpose.

The document was prepared in accordance with HNF-2353, Section 4.3, Attachment B, "Calculation Note Format and Preparation Instructions".

A I] [1] Concurrence

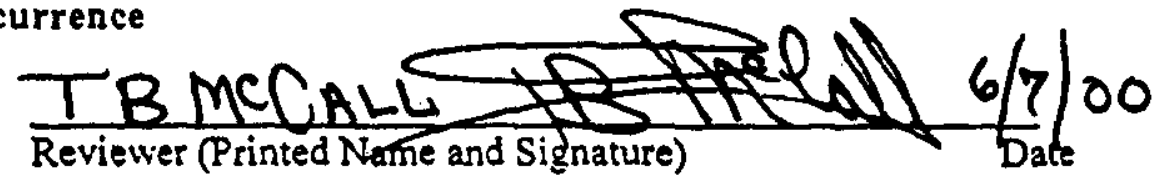

